\title{
ISPN 2021 Virtual Meeting of the International Society for Pediatric Neurosurgery
}

\author{
Virtual Meeting, 5 - 7 November 2021
}

Published online: 30 October 2021

๑) Springer-Verlag GmbH Germany, part of Springer Nature 2021

ISPN 2021 President: Francisco Salomao

ISPN 2021 Scientific Chair: Francesco Sala

ISPN 2021 Scientific Co-Chair: Abhaya Kulkarni

\section{Platform Abstracts}

PF-001

\section{Antenatal diagnosis and treatment \\ Antenatal Neurosonographic Diagnosis of Posterior Fossa Cyst: An Institutional Experience}

Harsha Shashank Ramdurg, Adinarayan Makam

Adi Advanced Centre for Fetal Care, Sparsh Hospital, Bengaluru, Karnataka, India

OBJECTIVE: Neurosonographic diagnosis of posterior fossa malformations in the prenatal period is a challenge, having major implications for the counseling and followup of pregnant women. The objective of this study was to review aspects of the ultrasound evaluation of the fetal posterior fossa and to describe the relevant ultrasound findings of the main posterior fossa malformations that can affect the foetus.

MATERIAL-METHODS: This was a retrospective study of fetuses with posterior fossa cyst, carried out between 2017 and 2019 in a tertiary level referral center for prenatal ultrasound diagnosis. All fetuses underwent multiplanar neurosonography using GE Voluson E10 ultrasound machine.

RESULTS: During the study period, 9600 foetuses were examined. 178 scans had neurological abnormalities. 28 cases had posterior fossa cysts (referral bias). The mean age of patients was 26.4 years (range 22- 34 years). The mean gestational age of 20.72 weeks (range, 19-26) weeks. Sonographic diagnoses (Blake's pouch cyst, $\mathrm{n}=7$; Dandy - Walker malformation, $\mathrm{n}=7$; Vermian hypoplasia, $\mathrm{n}=7$;
Megacisterna magna, $n=4$; Arachnoid cyst, $n=3$ ). Associated anomalies were in the form of ventriculomegaly in 4 cases and tetralogy of fallot in 1 case.

Dandy-Walker malformation were diagnosed on the basis of the classic triad: complete or partial agenesis of the vermis, cystic dilatation of the fourth ventricle, and torcular elevation. Vermian-cerebellar hypoplasia had a normal-sized posterior fossa, varying degrees of vermian and cerebellar hypoplasia. Mega cisterna magna were diagnosed as cystic mass with communication to subarachnoid space. Blake pouch cyst were characterized by communication between the cisterna magna and fourth ventricle, although the vermis and fastigium were normal.

CONCLUSION: An enlarged posterior fossa requires specific diagnoses for the best possible counseling. Isolated megacisterna magna and Blake's pouch cyst can either resolve or be normal variants, and need to be distinguished from dandy walker malformation and vermian hypoplasias which have poorer prognosis.

Keywords: Neurosongraphy, Posterior fossa cyst, Blakes pouch cyst, Dandy Walker Malformation, Arachnoid cyst, Mega cisterna magna

\section{PF-002}

Antenatal diagnosis and treatment

Prenatal hydrocephalus: reliability of radiological diagnosis and impact at one year

Olivier Klein ${ }^{1}$, Julien Todeschi ${ }^{1}$, Irene Stella ${ }^{1}$, Matthieu Helleringer $^{1}$, Anthony Joud ${ }^{1}$, Pascale Bach ${ }^{2}$

${ }^{1}$ Department of Pediatric Neurosurgery, Universite de Lorraine, Vandoeuvre les Nancy, France

${ }^{2}$ Maternite Regionale Universitaire, Nancy, France 
OBJECTIVE: The antenatal distinction between hydrocephalus and ventriculomegaly is sometimes difficult to assess. The aim of this study was to analyze the reliability of antenatal radiological criteria for hydrocephalus and the clinical condition of these children at 1 year.

MATERIAL-METHODS: This observational, retrospective, monocentric study included radiological suspicion of antenatal hydrocephalus from January 2007 to January 2019. Data were collected from the files of our multidisciplinary prenatal diagnostic center (center of reference, same institution). Hydrocephalus has been defined radiologically as macrocrania associated with decrease in the subarachnoid spaces and progressive triventricular dilation with obstructive features.

RESULTS: In this cohort, 207 ventricular dilations were found, including $37(17,9 \%)$ labeled hydrocephalus. The sex ratio $\mathrm{M} / \mathrm{F}$ was 0.84 with a diagnosis made on average at 23.5 weeks of gestation (WG) \pm 5.23 . An associated pathology was found in 23 cases: 17 cerebral malformations and 6 neural tube defects or dysraphism. The ventricular size was evaluated on average at $21.3 \mathrm{~cm} \pm 5.7$ [95\% CI 19.2-23.4]. Termination of pregnancy was requested and accepted in 22 cases $(59,5 \%)$. The remaining 15 children were born at 35.6 $\mathrm{WG} \pm 3.85$ with a head circumference at birth estimated at $39.3 \mathrm{~cm} \pm 6,88$ [IC $95 \% 35,2-43,3$ ]. Surgery was performed in 11 of them ( 7 shunt, 3 endoscopic third ventriculostomy, 1 intrauterine surgery for myelomeningocele closure). Three children died early and in one infant the diagnosis of ventriculomegaly was eventually made. The one-year psychomotor development of the operated patients was unfavorable in 8 cases ( 1 death, 7 delayed acquisitions) while 3 had a favorable development.

CONCLUSION: The diagnosis of antenatal hydrocephalus can be evoked thanks to precise radiological criteria. Nevertheless, the evolution and the prognosis of this often serious condition remains dependent on the associated pathologies and can sometimes be by itself associated with a non-favorable cognitive development.

Keywords: prenatal diagnosis, pediatric neurosurgery, hydrocephalus, outcome

\section{PF-003}

\section{Antenatal diagnosis and treatment Neuronal Loss in myelomeningocele during gestation, study on 186 cases of fetuses autopsy}

\author{
Selima Ben Miled ${ }^{1}$, Julien Stirnemann ${ }^{1}$, Syril James $^{2}$ \\ ${ }^{1}$ Department of Obstetrics and Maternal-Fetal Medi- \\ cine, Necker-Enfants Malades Hospital, AP-HP and Paris \\ Descartes University
}

${ }^{2}$ Department of Pediatric Neurosurgery, Necker-Enfants Malades Hospital, AP-HP and Paris Descartes University

OBJECTIVE: We collected all macroscopic findings from fetopathological and neuropathological examination. In this study, we wanted to show that the exposed spinal cord is significantly damaged early in pregnancy: even in the earliest cases, and also that the spinal injury extends cranially with a significant reduction in motor neurons in the adjacent cord right above the defect,

MATERIAL-METHODS: We retrospectively collected all post-mortem cases of spinal defect following termination of pregnancy (TOP) and confirmed by pathological examination from 1998 to 2018. We collected 186 cases of fetuses who had an autopsy confirming MMC $\leq \mathrm{T} 1$ and $>\mathrm{S} 1$. We aimed to compare the number of alpha motor neurons on spinal cord of MMC fetuses with controls. Among the MMC, the slide with the largest amount of ventral horn was chosen for counting. All counts were conducted on HE staining and only alpha motor neurons were counted.

RESULTS: Motor neuron counts in MMC cases were matched for gestational age and spinal level with $\mathrm{N}=41$ controls $(\mathrm{N}=7, \mathrm{~N}=8, \mathrm{~N}=9$, and $\mathrm{N}=17$ controls at $\leq 16$ weeks, 16-20 weeks, 20-24 weeks and $>24$ weeks respectively), yielding to a total of 109 tiered samples of spinal cord.

CONCLUSION: In MMC, significant neuronal loss is visible very early in pregnancy, progresses throughout gestation, and extends cranially. Early intrauterine interventions ( $<16$ weeks) may rescue the remaining 17\% normal motor neurons within the MMC and prevent the cranial extension of neuronal loss.

Keywords: myelomeningocele, Chiari, alpha motor neurons, intrauterine interventions.

\section{PF-004}

Antenatal diagnosis and treatment

Antenatal surgery for myelomeningocele: influencing factors and comparison between two initial series in different socio-demographic scenarios

Eduardo Jucá ${ }^{1}$, Federico Di Rocco ${ }^{6}$, Edson Lucena ${ }^{2}$, Timothée De Saint Denis ${ }^{4}$, Herlânio Costa ${ }^{2}$, Aldo Melo ${ }^{3}$, Fernanda Castro ${ }^{2}$, Jean Marie Jouannic ${ }^{5}$, Denise Francelino ${ }^{2}$, Catherine Garel ${ }^{5}$, Renata Jucá ${ }^{2}$, Michel Zerah ${ }^{4}$

${ }^{1}$ Department of Pediatric Neurosurgery, Hospital Infantil Albert Sabin, Fortaleza, Brazil

${ }^{2}$ Maternidade Escola Assis Chateaubriand, Universidade Federal do Ceará, Fortaleza, Brasil

${ }^{3}$ Departament of Surgery and Anatomy, Universidade Federal do Ceará, Fortaleza, Brasil 
${ }^{4}$ Department of Pediatric Neurosurgery, Hôpital Necker Enfants Malades, Paris, France

${ }^{5}$ Department of Gynecology and Obstetrics, Hôpital Trousseau, Paris, France

${ }^{6}$ Department of Pediatric Neurosurgery, Hôpital FemmeMère-Enfant, Lyon, France

${ }^{7}$ Department of Physical Therapy, Universidade Federal do Ceará, Fortaleza, Brazil

OBJECTIVE: Aim of this study is to compare initial results of myelomeningocele antenatal surgical repair in two services from different socio-demographic scenarios and identify influencing issues affecting the programs' evolution on each side.

MATERIAL-METHODS: We performed an observational retrospective analysis of initial series of myelomeningocele antenatal surgical repair in Paris (France) and in Fortaleza (Brazil). Data was collected from both services' records for comparison and analysis.

RESULTS: Paris has started its program four years ago and performed 17 operations, while Fortaleza had 16 surgical cases in one year. Both services had 15 children born so far. Paris had 133 referred cases, with 20\% of misdiagnosis, 10 non eligible cases according to inclusion criteria, 55 cases with parental choice to termination of pregnancy (TOP) and 18 cases with parental refusal to antenatal intervention, resulting in postnatal surgery. Fortaleza had 19 referred cases and 3 were non eligible. There was no TOP nor parental refusal to antenatal intervention. Average gestational age at birth was 34.4 weeks in Paris and 34,04 weeks in Fortaleza. Neither services had child mortality. Paris had no mother morbidity, while there was a post-op pneumonia and a leg venous thrombosis in Fortaleza. No child needed intubation after birth in Paris, whereas 3 did so, transiently, in Fortaleza. Paris had 2 children shunted for hydrocephalus and Fortaleza had one shunted child so far.

CONCLUSION: Legal and religious issues can explain the differences concerning amount of referrals and yearly performed surgeries, once TOP is not allowed in Brazil. Results are comparable in both services and overall satisfactory when compared to MOMS study standards. Apparent higher mother and child morbidity in Brazil could be explained by general healthcare conditions disparities. Antenatal myelomeningocele repair is feasible and potentially advantageous in different geographical scenarios, although social, demographic and legal issues influence a service's evolution.

Keywords: Myelomeningocele, Antenatal surgery, results

\section{PF-005}

\section{Dysraphism}

Intraoperative neuromonitoring for tethered cord surgery in infants- Challenges, nuances and outcome

Suhas Udayakumaran ${ }^{1}$, Nivedita.s.nair S Nair ${ }^{2}$, Mathew George $^{3}$, Siby Gopinath ${ }^{2}$, Sri Valli Puthi ${ }^{2}$

${ }^{1}$ Division of Paediatric Neurosurgery, Amrita University, Kochi, India

${ }^{2}$ Division of Electrophysiology, Amrita University, Kochi, India

${ }^{3}$ Department of Anaesthesia, Amrita University, Kochi, India

OBJECTIVE: To evaluate the efficacy and safety of intraoperative neuromonitoring (IONM) in surgery for tethered cord in infants.

MATERIAL-METHODS: The study included eighty seven infants who underwent surgery for closed spinal dysraphism under IONM. Their preoperative neurological and urological statuses were compared with postoperative status clinically. The study design was prospective, and the duration of the study was from January 2011 to February 2020. IONM was performed (MEP and direct stimulation) with Xltek Protektor 32 IOM system, Natus Neurology/medical inc. Middleton, USA. Statistical analysis in the form of Chi-square is conducted using SPSS.

RESULTS: Overall, among 87 patients, clinical improvement was seen in $28(28 / 29)$ patients with motor deficits, 23 (23/24) with bladder deficits and 18 (18/19) with bowel deficits. The monitorability for motor and sphincter was $97.3 \%$ and $90.7 \%$ respectively. The sensitivity of IONM in predicting new neurological deficit was $90.9 \%$, whereas the specificity was $98.3 \%$. The positive predictive value was $100 \%$, with the negative predictive value of $97.3 \%$. The diagnostic accuracy was $98.8 \%$. There were no complications in this cohort directly related to the IONM procedure.

CONCLUSION: The study has highlighted that the use of IONM in infants is efficacious and safe. However, IONM is specific but not very sensitive in diagnosing neural injury during detethering of tethered cord in infants. IONM has an excellent diagnostic accuracy even in very young for any potential neurological deficit.

Keywords: Intraoperative neuromonitoring, Tethered cord, Infants, Spinal dysraphism

\section{PF-006}

\section{Epilepsy}

Leptomeningeal capillary malformation area and epilepsy severity in Sturge-Weber syndrome

Hidenori Sugano, Kazuaki Shimoji, Masakazu Miyajima, Hajime Arai 
Department of Neurosurgery, Juntendo, University, Tokyo, Japan

OBJECTIVE: To test the hypothesis that the severity of neurological damage in Sturge-Weber syndrome (SWS) is based on the extent of leptomeningeal capillary malformation (LCM), cross-sectional analyses of seizures, electroencephalogram (EEG) severity, and surgical requirements were performed in a retrospective cohort based on the extent of LCM. MATERIAL-METHODS: We classified 112 patients into the following four groups according to LCM distribution as determined by SWI and FLAIR-Gd-enhanced MRI: (1) Bilateral (Bil), (2) Hemispheric (Hemi), (3) Multilobar (Multi), and (4) Single lobe. We compared age at seizure onset, seizure semiology and its incidence, and EEG findings. Surgeries were indicated for patients with antiepileptic drug resistance, progressive cerebral atrophy, and cognitive decline. Surgical requirements were evaluated among the groups. The SWS-neurological score (SWS-NS) was applied for evaluation of therapeutic efficacy.

RESULTS: Both the Bil and the Hemi groups were characterized by early onset, frequent, focal-to-bilateral tonic-clonic seizures, and status epilepticus. No significant differences were observed on EEG among groups. Regarding Bil patients, $77.8 \%$ required corpus callosotomy and $88.1 \%$ of Hemi cases required hemispherotomy. The Multi group required surgery in $50.0 \%$ of the cases. Seizure and cognitive sub-scores of SWS-NS were improved in the Hemi and Multi groups following surgical intervention.

CONCLUSION: Our study demonstrated that extensive LCM was strongly associated with seizure and cognitive severity.

Keywords: Sturge-Weber syndrome, Leptomeningeal capillary malformation, outcome, epilepsy surgery

\section{PF-007}

\section{Epilepsy}

Optimized SEEG-guided radiofrequency thermocoagulation in the treatment of pediatric hypothalamic hamartomas

\section{Rui Zhao, Hao Li}

Department of Neurosurgery, Children's Hospital of Fudan University, Shanghai, China

OBJECTIVE: Hypothalamic hamartomas (HHs) are rare malformations and the treatment is challenging. This study investigated the role of one-stage stereo-array radiofrequency thermocoagulation (RF-TC) based on stereotactic electroencephalography (SEEG) in pediatric HHs.
MATERIAL-METHODS: We analyzed the clinical data of 28 patients with $\mathrm{HH}$ who underwent SEEG exploration between November 2015 and April 2019. A high-density focal stereo-array electrode implantation strategy was developed in this study. SEEG-guided bipolar coagulations were performed between two contiguous contacts of the same electrode, or between two adjacent contacts of different electrodes. Patients with HHs were divided into two groups based on size of the hamartoma (maximum diameter $<15 \mathrm{~mm}$ vs maximum diameter $\geq 15 \mathrm{~mm}$ ). Numbers of implanted electrodes and contacts used for thermocoagulation were compared between two groups. Seizure outcome was evaluated according to Engel's classification.

RESULTS: Surgical procedures were well tolerated and no repeated surgery was performed. The median number of electrodes implanted in the two groups $(<15 \mathrm{~mm}$ vs $\geq 15 \mathrm{~mm}$ ) was significantly different ( 2 vs $6, p=0.0009$ ), as well as the median number of contacts where RF-TC was applied ( 7 vs $40, p=0.0006$ ). Moreover, the number of implanted electrodes and contacts used for thermocoagulation were positively correlated with the hamartoma volume (Spearman's rho $=0.7074, \mathrm{p}<0.0001$ and Spearman's rho $=0.7435, \mathrm{p}<0.0001$, respectively). The overall seizure-free rate was $82.1 \%$, with $92.9 \%$ of the patients having favorable (Engel class I and II) outcomes for at least 12 months of follow-up. Seizure outcomes between two groups were not statistically significant $(\mathrm{p}=0.3138)$ and no significant correlation between hamartoma volume and seizure outcomes $(\mathrm{p}=0.9010)$ was identified.

CONCLUSION: One-stage high-density focal stereo-array SEEG-guided RF-TC using cross-bonded electrode contacts for ablation range expansion is a safe and effective surgical approach for children with HHs.

Keywords: children; stereoelectroencephalography; hypothalamic hamartoma; radiofrequency thermocoagulation

\section{PF-008}

\section{Epilepsy \\ Complications' analysis and surgical factors affecting safety of SEEG in pediatric patients}

Ioannis $\mathrm{N}$ Mavridis ${ }^{1}$, William $\mathrm{B} \mathrm{Lo}^{1}$, Buddhika Wimalachandra $^{1}$, Sunny Philip ${ }^{2}$, Shakti Agrawal ${ }^{2}$, Caroline $\mathrm{Scott}^{3}$, Daren Martin Lamb ${ }^{3}$, Bryony $\mathrm{Carr}^{3}$, Peter Bill ${ }^{3}$, Andrew Lawley ${ }^{3}$, Stefano Seri ${ }^{3}$, Anthony Richard Walsh ${ }^{1}$

${ }^{1}$ Department of Neurosurgery, Birmingham Children's Hospital, Birmingham, West Midlands, England, United Kingdom

${ }^{2}$ Department of Neurology, Birmingham Children's Hospital, Birmingham, West Midlands, England, United Kingdom 
${ }^{3}$ Department of Neurophysiology, Birmingham Children's Hospital, Birmingham, West Midlands, England, United Kingdom

OBJECTIVE: Stereoelectroencephalography (SEEG) is used increasingly to identify epileptogenic foci as part of epilepsy surgery work up, facilitated by robot-assistance. We aimed to analyze our complications and study whether a number of different surgical parameters were associated with the complication rate of pediatric SEEG.

MATERIAL-METHODS: Sixty three pediatric patients, 24 boys and 39 girls (4-18 years of age), underwent 64 SEEG procedures with a total of 726 implants. The Leksell stereotactic system ${ }^{\circledR}$ was used in all implantations. In the first 16 children, implantation technique was frame-based. From the 17th child onward, the neuromate ${ }^{\circledR}$ robotic system (Renishaw plc) for stereotactic neurosurgery was used. All patients had preoperative, intraoperative and postoperative imaging studies. The surgical parameters studied included the laterality of implantation (unilateral or bilateral), side of implantation (right or left), number of implanted electrodes, and duration of implantation (time from implantation to explantation).

RESULTS: The overall complication rate was 6.3\% (4 patients). The observed complications included: asymptomatic extradural hematoma (1), retained electrode (1), and transient neurological deficits (2). Overall, 3.1\% (2) of patients required repeat surgery because of a surgical complication and one patient (1.6\%) underwent craniotomy for this reason. All complications were either transient or treatable and no patient developed permanent sequelae. The complication rate for patients with left-sided electrodes was remarkably higher compared to those with right-sided electrodes, namely $11.1 \%$ vs $3.3 \%(\chi 2=1.162, p=0.281)$. It was slightly higher for those with $\geq 10$ implants $(6.8 \%$ vs $5 \%)$ and for those implanted for $\leq 5$ days ( $6.9 \%$ vs $5.7 \%$ ).

CONCLUSION: SEEG is safe and its complications, more frequent with left-sided implants, are usually either transient or reversible. Skull drilling, preservation of electrode integrity during recording and electrodes' explantation, are critical steps for the complications' avoidance. Regular neurological examination and postoperative imaging are keys for their early detection.

Keywords: complications, implants, left cerebral hemisphere, safety, SEEG procedure, surgical factors

\section{PF-009}

\section{Epilepsy}

Intraopeative Brain Mapping to Identify Corticospinal Projections during Epilepsy Surgery in Patient with Hemiparesis and Drug-Resistant Epilepsy
Hsin Hung Chen ${ }^{1}$, Tsui Fen Yang ${ }^{2}$

${ }^{1}$ Division of Pediatric Neurosurgery, The Neurological Institute, Taipei Veterans General Hospital

${ }^{2}$ Department of Physical Medicine \& Rehabilitation

OBJECTIVE: Resective epilepsy surgery for better seizure control is not without the risk of creating new postoperative deficits. The purpose of this study is to determine corticospinal organization using intraoperative neurophysiologic monitoring (IONM) and the impact on surgical decision making for patients with congenital hemiparesis and intractable epilepsy who underwent resective epilepsy surgery.

MATERIAL-METHODS: Thirty-two patients, aged 0.6-39, with intractable epilepsy were enrolled. Etiologies included malformation of cortical development mostly, and gliosis from various causes, congenital infarction and tuberous sclerosis complex as well. Transcranial stimulation (TCS) was achieved using a pair of cork screws at $\mathrm{Cz}$ and $\mathrm{C} 3 / \mathrm{C} 4$, respectively. Multipulse train stimulation was delivered, with simultaneous recordings from bilateral abductor pollicis brevis and abductor halluces, to determine the corticospinal projection pattern of the paretic limbs for each patient before resection.

RESULTS: The above mapping techniques revealed ipsilateral corticospinal projections from the contralesional hemisphere to target muscles in the paretic limbs in five patients, projections from both hemispheres to target muscles in ten, and preserved crossing projections from the affected hemisphere in seventeen. Based on above findings, 21 of them underwent lesionectomy, 5 of them hemispherotomy and 6 received quadrantic disconnection surgery. Twenty three patients were either seizure free or almost seizure free according to Engel epilepsy surgery outcome scale during follow up period. Six experienced transient new motor weakness, and 23 of them had either unchanged or improved use of the paretic limbs after op. There were 3 patients sustained long term weakness of the affected limbs in our series.

CONCLUSION: Using IONM to define the corticospinal projection pattern is a valuable technique that has significant impact on surgical decision making and can potentially replace preoperative fMRI and transcranial magnetic stimulation in epilepsy surgery, particularly for younger and uncooperative patients.

Keywords: Drug-resistant epilepsy, hemiparesis, intraoperative neuromonitoring, epilepsy surgery, corticospinal projections

\section{PF-010}

\section{Epilepsy}

Limits of Epilepsy Surgery in Children 
Helio Rubens Machado ${ }^{1}$, Marcelo Volpon Santos ${ }^{1}$, Ursula Thomé $^{2}$, Felipe Chodraui ${ }^{1}$, Camila Araujo ${ }^{2}$, Larissa Batista ${ }^{2}$, Ana Hamad ${ }^{2}$

${ }^{1}$ Division of Pediatric Neurosurgery, Ribeirao Preto Medical School, University of Sao Paulo, Ribeirao Preto, Brazil ${ }^{2}$ Cirep, Center for Epilepsy Surgery in Children, Ribeirao Preto Medical School, University of Sao Paulo, Ribeirao Preto, Brazil

OBJECTIVE: Epilepsy surgery in children differs from adults in many aspects. Although temporal lobe surgery is common in adults, extra-temporal surgery predominates in children. Children are particularly prone to harbor hemispheric diseases (typically Rasmussen encephalitis, among others) and cortical dysplasia (CD). Also, CD frequently spans the central region (Rolandic area), and this poses a challenge concerning resective surgery in these cases, which is to avoid further neurological deficits in these already compromised children. Our goal is to analyze a series of children under the age of 18 treated at our institution with epileptogenic lesions in eloquent areas (central region, language areas and insula).

MATERIAL-METHODS: Our series comprises patients operated regularly in a dedicated pediatric Epilepsy Surgery Center, since 1996. For the sake of uniformity, we excluded all patients submitted to hemispheric or disconnective surgery. All patients were investigated through an established protocol by a multidisciplinary team. All children were submitted to MR (3T) and video-telemetry. Decisions regarding the surgical indication or the modality of invasive monitoring were taken on a weekly clinical meeting, with the whole team involved.

RESULTS: Among 701 children operated on during the period 1996-March 2020, 86 cases fulfilled our inclusion criteria. Most frequently, the surgical procedure was guided by neuronavigation alone, followed by electrocorticography (ECoG), either acute or chronic, and, more recently, stereoelectroencephalography (SEEG), allowing for a variable period of monitoring and stimulation of motor/language areas in the ward. The majority of patients had $\mathrm{CD}$, followed by tubers, tumors and cavernomas.

CONCLUSION: Epilepsy surgery in children is frequently related to cortical dysplasia and other lesions in close relation to eloquent areas, mainly in the central region or insula. They can be successfully operated on, although in some cases a temporary deficit is observed, which gradually subsides.

Keywords: epilepsy surgery, children, cortical dysplasia, insula, language area, invasive monitoring

\section{PF-011}

Epilepsy
Epilepsy surgery in children under 3 years of age: surgical and developmental outcomes

Masaki Iwasaki ${ }^{1}$, Keiya Iijima ${ }^{1}$, Takahiro Kawashima ${ }^{2}$, Hisateru Tachimori ${ }^{2}$, Yutaro Takayama ${ }^{1}$, Yuiko Kimura ${ }^{1}$, Yuu Kaneko ${ }^{1}$, Naoki Ikegaya ${ }^{3}$, Noriko Sumitomo ${ }^{4}$, Takashi Saito $^{4}$, Eiji Nakagawa ${ }^{4}$, Akio Takahashi ${ }^{5}$, Kenji Sugai ${ }^{4}$, Taisuke Otsuki ${ }^{6}$

${ }^{1}$ Department of Neurosurgery, National Center Hospital, National Center of Neurology and Psychiatry, Kodaira, Japan

${ }^{2}$ Department of Clinical Epidemiology, Translational Medical Center, National Center of Neurology and Psychiatry, Kodaira, Japan

${ }^{3}$ Department of Neurosurgery, Yokohama City University Hospital, Yokohama, Japan.

${ }^{4}$ Department of Child Neurology, National Center Hospital, National Center of Neurology and Psychiatry, Kodaira, Japan

${ }^{5}$ Department of Neurosurgery, Shibukawa Medical Center, Shibukawa, Japan

${ }^{6}$ Epilepsy Centre Bethel Japan, Iwanuma, Japan

BACKGROUND AND AIM: Pediatric epilepsy surgery is known to be effective, but early surgery in infancy is not well characterized. Large cortical dysplasia, such as hemimegalencephaly, can cause refractory epilepsy shortly after birth and early surgical intervention is indicated. However, the complication rate of early pediatric surgery is significant. This study assessed the risk benefit of early pediatric epilepsy surgery on developmental outcomes.

METHODS: This is a retrospective descriptive study of 75 patients who underwent the first curative epilepsy surgery under the age of 3 years in our institution between 2006 and 2019 with a minimum of one-year follow-up of seizure and developmental outcomes. Clinical information including surgical complications, seizure outcomes, and developmental quotient (DQ) was collected from medical records. The effects of clinical factors on DQ at one year after surgery were evaluated.

RESULTS: The median age at surgery was 6 months, peaking at between 3 and 4 months. Operative procedures included 27 cases of hemispherotomy and 19 cases of multilobar surgery. Seizure freedom was achieved in $82.7 \%$ of patients at one year and $71.0 \%$ at mean 62.8 months follow-up. The number of antiseizure medications (ASMs) decreased significantly after surgery and 19 patients (30.6\%) had discontinued ASMs at the last follow-up. Postoperative complications requiring cerebrospinal fluid diversion surgery, such as hydrocephalus and cyst formation, were observed in 13 patients (17.3\%). Mean DQ was $74.2 \pm 34.3$ preoperatively, $60.3 \pm 23.3$ at one year after surgery, and $53.4 \pm 25.1$ at the last follow-up. Multiple regression 
analysis revealed that one-year postoperative DQ was significantly influenced by preoperative DQ and postoperative seizure freedom, but not by the occurrence of such surgical complications.

CONCLUSIONS: Early pediatric epilepsy surgery has acceptable risk-benefit balance. Seizure control after surgery is important for postoperative development.

Keywords: epileptic encephalopathy, hemispherotomy, hemimegalencephaly, malformation of cortical development, developmental quotient

\section{PF-012}

\section{Epilepsy}

Intracranial neuromodulation (deep brain stimulation and responsive neurostimulation) in children with drugresistant epilepsy: a systematic review

Mehdi Khan ${ }^{1}$, Jaber Paktiawal ${ }^{2}$, Rory J Piper $^{3}$, Aswin Chari $^{3}$, Martin M Tisdall ${ }^{3}$

${ }^{1}$ University College London Medical School, London, UK; Department of Neurosurgery, Great Ormond Street Hospital, London, UK

${ }^{2}$ Medical University Pleven, Pleven, Bulgaria; Department of Neurosurgery, Great Ormond Street Hospital, London, UK

${ }^{3}$ Department of Neurosurgery, Great Ormond Street Hospital, London, UK; Developmental Neuroscience, Great Ormond Street Institute of Child Health, University College London, London, UK

BACKGROUND AND AIM: In children with drug-resistant epilepsy (DRE), resective, ablative and disconnective surgery may not be feasible or may fail. Deep brain stimulation (DBS) and responsive neurostimulation (RNS) may be viable treatment options, however, evidence for their efficacies in children is currently limited. This systematic review aims to summarize the DBS and RNS literature for the treatment of DRE in children. We focus on the currently available data for reported indications, neuromodulation targets, clinical efficacy and safety outcomes.

METHODS: PRISMA guidelines were adhered to throughout this study. Five electronic databases were searched from their inception to February 19, 2021. Inclusion criteria were 1) studies with at least one pediatric patient (age $<19$ years) undergoing DBS and/or RNS for DRE; 2) retrospective and prospective studies. Exclusion criteria were 1) letters, abstracts, reviews; 2) non-English language studies.

RESULTS: A total of 35 studies were selected, identifying 72 and 46 patients undergoing DBS and RNS respectively (ages 4-18 years). Overall, 75\% of patients had a seizure reduction $>50 \%$ following DBS at a median follow-up of
14 months (range 1-100 months). In exploratory univariate analysis of factors associated with favorable response, follow-up duration was shorter in those with a favorable response ( 18 vs 33 months, $\mathrm{p}<0.05$ ). In the RNS cohort, $62.5 \%$ of patients had a seizure reduction $>50 \%$ following RNS, at a median follow-up of 22 months (range 5-39 months). Of interest, $83.3 \%$ of patients who had $>50 \%$ reduction in seizures actually had a $>75 \%$ reduction.

CONCLUSIONS: Both DBS and RNS showed favorable response rates, indicating both techniques should be considered in pediatric patients with DRE. Research needs in this area would be addressed by conducting high-quality clinical trials and establishing an international registry for pediatric neuromodulation, ensuring the robust prospective collection of predictive variables and outcomes.

Keywords: Deep brain stimulation, Responsive NeuroStimulation, Drug-resistant epilepsy, Pediatric

\section{PF-013}

\section{Epilepsy}

Epileptogenic modulation index and synchronization in hypsarrhythmia of West syndrome secondary to perinatal arterial ischemic stroke

Hiroharu Suzuki ${ }^{1}$, Hiroshi Otsubo ${ }^{2}$, Mahendranath Moharir ${ }^{2}$ ${ }^{1}$ Division of Neurology, Department of Pediatrics, The Hospital for Sick Children, Toronto, Canada; Department of Neurosurgery, Juntendo University, Tokyo, Japan ${ }^{2}$ Division of Neurology, Department of Pediatrics, The Hospital for Sick Children, Toronto, Canada

BACKGROUND AND AIM: Perinatal arterial ischemic stroke (PAIS) is associated with epileptic spasms of West syndrome (WS) and long term Focal epilepsy (FE). The mechanism of epileptogenic network generation causing hypsarrhythmia of WS is unknown. We hypothesized that Modulation index (MI) [strength of phase-amplitude coupling] and Synchronization likelihood (SL) [degree of connectivity] could interrogate the epileptogenic network in hypsarrhythmia of WS secondary to PAIS.

METHODS: We analyzed interictal scalp electroencephalography (EEG) in $10 \mathrm{WS}$ and $11 \mathrm{FE}$ patients with unilateral PAIS. MI between gamma $(30-70 \mathrm{~Hz})$ and slow waves (3-4 Hz) was calculated to measure phase-amplitude coupling. SL between electrode pairs was analyzed in 9-frequency bands (5-delta $(0.5-1 \mathrm{~Hz} / 1-2 \mathrm{~Hz} / 2-3 \mathrm{~Hz} / 3-4 \mathrm{~Hz} / 0.5$ $-4 \mathrm{~Hz}$ ), theta, alpha, beta, gamma) to examine inter- and intra-hemispheric connectivity.

RESULTS: MI was higher in affected hemispheres in WS $(\mathrm{p}=0.006)$; no differences observed in FE. Inter-hemispheric SL of 3-delta (1-2 Hz/2-3 Hz/3-4 Hz), theta, alpha, beta, 
gamma bands was significantly higher in WS $(\mathrm{p}<0.001)$. In WS, modified Z-Score of intra-hemispheric SL values in 3-delta (1-2 Hz/2-3 Hz/3-4 Hz), theta, alpha, beta and gamma in the affected hemispheres were significantly higher than those in the unaffected hemispheres $(\mathrm{p}<0.001)$ as well as $0.5-4 \mathrm{~Hz}(\mathrm{p}=0.004)$.

CONCLUSIONS: The significantly higher modulation in affected hemisphere and stronger inter- and intra-hemispheric connectivity generate hypsarrhythmia of WS secondary to PAIS. Epileptogenic cortical-subcortical transcallosal networks from affected hemisphere post-PAIS provokes infantile spasms.

Keywords: West syndrome, Focal epilepsy, Modulation index, Phase-amplitude coupling, Synchronization likelihood, Functional connectivity

\section{PF-014}

\section{Epilepsy}

Drug-resistant focal epilepsy in children is associated with increased network modal controllability of the whole brain and epileptogenic regions

Aswin Chari ${ }^{1}$, Kiran Seunarine ${ }^{2}$, Xiaosong $\mathrm{He}^{3}$, Chris Clark $^{2}$, Richard Rosch ${ }^{2}$, Danielle Bassett ${ }^{3}$, Rod Scott ${ }^{2}$, Martin Tisdall ${ }^{1}$

${ }^{1}$ Department of Neurosurgery, Great Ormond Street Hospital, London, UK

${ }^{2}$ Developmental Neuroscience, Great Ormond Street Institute for Child Health, University College London, London, UK

${ }^{3}$ Department of Bioengineering, University of Pennsylvania, Philadelphia, PA, USA

BACKGROUND AND AIM: Network science can aid our understanding of drug-resistant epilepsy in children. Network control theory can model functional dynamics from the structural (diffusion MRI) data and may provide insight into the disruption of brain dynamics in drug-resistant epilepsy. We sought to assess differences in network modal controllability, defined as the ability of a brain region to drive the system to difficult-to-reach neurophysiological states, between healthy controls and children with drug-resistant epilepsy.

METHODS: A total of 79 patients and 16 healthy controls were included. All patients had drug-resistant epilepsy necessitating presurgical evaluation and were split into 2 groups based on whether they underwent surgical resection (resection group, $\mathrm{n}=52$ ) or vagal nerve stimulator implantation (VNS group, $\mathrm{n}=27$ ). Structural connectivity networks were constructed using probabilistic tractography. Nodes in the network were atlas-based cortical and subcortical parcels (253 parcels in each patient) and edges were the number of streamlines connecting the parcels. Modal controllability of each parcel and the mean of all 253 parcels were calculated. RESULTS: Both patient groups had a higher mean modal controllability (resection: $p=0.003$, VNS: $p=1 \times 10-6$ ) compared to controls. In both groups, there was lower modal controllability in select parcels of the thalamus and posterior cingulate cortices, both considered part of the 'structural core' of the brain.

In the resection group, resected parcels had a higher modal controllability than the rest of the brain, findings that were statistically significant only in patients that were seizure free following surgery $(\mathrm{p}=6 \times 10-4)$.

CONCLUSIONS: We identified increases in network modal controllability in children with drug-resistant epilepsy compared to controls. We found increased modal controllability of resected regions, suggesting that these regions can preferentially reach distant neurophysiological states such as seizures. The degree of this abnormality was correlated with seizure free outcomes.

Keywords: Epilepsy, Resective Surgery, Network neuroscience

\section{PF-015}

\section{Functional}

Selective Dorsal Rhizotomy: Post-operative Requirements for Orthopaedic Surgery

Isabel Tulloch, James Robins, Adam Galloway, Catherine Wilsmore, Kate Mccune, Andie Mulkeen, Katie Davis, Helen Bryant, Laura Deriu, Ian Smith, Rajib Lodh, John Goodden

Leeds Children's Hospital

OBJECTIVE: In recent years, there has been speculation as to whether Selective Dorsal Rhizotomy (SDR) alters the requirement for future orthopaedic bony or musculo-tendinous surgery. We have completed 125 SDR procedures at our centre, with more than six months of post-operative followup data having been collected for 110 of these patients. We have evaluated the frequency, timing and type of orthopaedic procedures that patients undergo following SDR procedures. MATERIAL-METHODS: Prospective data collected on children undergoing SDR since October 2012. All patients were GMFCS Level II or III. Patients are followed at six, twelve and twenty-four months post-operatively and then at five and ten years. Data collection included timing, and type of orthopaedic surgery.

RESULTS: Thirty (27\%) underwent orthopaedic procedures post-SDR - average age 7.2 years (4.4-13.8), M:F ratio 1.8:1. Median time between SDR \& orthopaedic surgery was 9-months (1-61). Full details of surgery types are 
available for presentation. No patients have required spinal surgery after SDR.

GMFCS Level II: 10/50 patients required orthopaedic surgery at a mean of 16.3 months post-SDR surgery. The mean age at orthopaedic surgery 6.9 years. 4 required combined bone and soft tissue surgery and 6 only needed soft tissue surgery.

GMFCS Level III: 20/60 patients required orthopaedic surgery at a mean of 12.3 months post-SDR surgery. The mean age at orthopaedic surgery was 7.4 years. Eight patients required combined bone and soft-tissue surgery, and 12 only needed soft-tissue surgery.

CONCLUSION: Our data provides an initial insight into orthopaedic surgery requirements after SDR. We have noted that GMFCS Level II children require less additional surgery and the average age at orthopaedic surgery was younger than eight years old - this finding will require long-term followup to determine whether this results in re-operation requirements later in life.

Keywords: Selective Dorsal Rhizotomy, Orthopaedic Surgery, Musculo-tendinous surgery, GMFCS, Post-operative Outcome

\section{PF-016}

\section{Functional}

Short-term alternation in gait analysis after single-level SDR guided by our newly-modified rhizotomy protocol in patients with GMFCS I and II

\author{
Wenbin Jiang ${ }^{1}$, Yan $\mathrm{Yu}^{2}$, Qijia Zhan ${ }^{1}$, Bo Xiao ${ }^{1}$, Shuyun \\ Jiang $^{2}$ \\ ${ }^{1}$ Department of Neurosurgery, Shanghai Children's Hospital, \\ Shanghai, China \\ ${ }^{2}$ Department of Gait analysis, Yueyang Hospital of Inte- \\ grated Traditional Chinese and Western Medicine, Shang- \\ hai, China
}

OBJECTIVE: To evaluate the short-term outcome of pediatric patients with spastic cerebral palsy $(\mathrm{CP})$ who underwent selective dorsal rhizotomy (SDR) guided by our newly-modified rhizotomy protocol with gait analysis (GA).

MATERIAL-METHODS: A total of 25 children with spastic CP operated in Shanghai Children's Hospital were retrospectively reviewed. All patients have undergone pre-operative and post-operative GA for evaluation. Changes in gait deviation index (GDI), kinematics of ankle, knee and hip as well as alternation in spatiotemporal parameters in both affected and unaffected limbs were evaluated.

RESULTS: Improvement was seen in the mean GDI of 43 affected sides, which increased from 57.48 to 61.24 $(\mathrm{P}<0.01)$. The maximum degree of ankle plantar flexion
(PF) decreased from 18.45 to $13.87(\mathrm{P}=0.001)$, maximum ankle dorsal-flexion (DF) increased from 1.85 to 7.89 $(\mathrm{P}<0.001)$, and the mean DF of the ankle at end of swing phase increased from -10.93 to $-8.39(\mathrm{P}<0.05)$. Mean flexion degree of knee at initial contact (IC) and at the end of swing phase decreased from 33.73 to $27.69(\mathrm{P}=0.001)$ and from 35.71 to $28.35(\mathrm{P}<0.001)$ respectively. 3 of 25 patients underwent 2 post-op GA. Mean maximum flexion of knee at IC decreased from 38.44 to 31.71 and then to 21.18 and mean flexion of knee at end of swing phase decreased from 41.14 to 33.98 and then to 22.27. However, walking velocity decreased from 119.79 to $101.24(\mathrm{P}<0.001)$, and the percentage of stance phase was longer after surgery $(67.92 \%$ vs $70.02, \mathrm{P}<0.05)$. No significant changes were found in unaffected limbs.

CONCLUSION: SDR guided by our newly-modified protocol with post-operative exercise was helpful to spastic $\mathrm{CP}$ patients. All patients presented with improvements in kinematic parameters in affected sides after SDR but some of the spatiotemporal parameters were worse than pre-op state. GDI of affected sides improved significantly after the surgery. The improvements tended to increase over time.

Keywords: spastic cerebral palsy, dorsal rhizotomy, outcome, gait analysis

\section{PF-017}

\section{Functional \\ Outcomes following SDR in children with bilateral spas- tic Cerebral Palsy and GMFCS Level IV}

Adikarige Haritha Dulanka Silva ${ }^{1}$, Claudia Craven ${ }^{1}$, Stephanie Cawker ${ }^{2}$, Deepti Chugh ${ }^{2}$, Belinda Crowe ${ }^{2}$, Lucinda Carr $^{2}$, Kristian Aquilina ${ }^{1}$

${ }^{1}$ Department of Paediatric Neurosurgery, Great Ormond Street Hospital for Children NHS Foundation Trust, London, United Kingdom

${ }^{2}$ Department of Paediatric Neurodisability, The Wolfson Neurodisability Service, Great Ormond Street Hospital for Children NHS Foundation Trust, London, United Kingdom

OBJECTIVE: Selective dorsal rhizotomy (SDR), an irreversible surgical procedure involving division of selected sensory nerve roots followed by intensive physiotherapy, was recently commissioned by NHS England for selected children with bilateral spastic cerebral palsy (CP) and Gross Motor Function Classification System (GMFCS) level II and III. Long term data regarding efficacy and outcomes following SDR in GMFCS IV children is limited. We assessed gross motor function before and after SDR in this cohort. MATERIAL-METHODS: A retrospective analysis of children with CP and GMFCS IV treated with SDR. All children 
were selected for SDR following multidisciplinary assessment and underwent SDR via single-level laminoplasty approach with intraoperative neurophysiological monitoring. All children underwent an individualised physiotherapy regimen that was specified for 24-months after surgery. Multidisciplinary evaluation was conducted pre-operatively, 6, 12 and 24-months after surgery. Primary outcomes were change in gross motor function, measured with GMFM-66 and change in GMFM-66 age-adjusted centiles.

RESULTS: A total of 33 children were identified (16 males). Of these, 29 patients had completed at least one assessment and 16 patients (mean age 6.13 years [range: $3.42-10.53$ ]) had completed assessments at all 4 time points.

A paired, repeated measures one-way ANOVA (with a Geisser-Greenhous's epsilon correction) was performed to test for significant differences in those 16 patients with complete data sets.

There were significant differences at pre-op, 6, 12 and 24-month post-op time-points in:

1. Mean GMFM-66 score $(\mathrm{p}<0.0001)$

2. Mean GMFM-66 age-adjusted centiles $(\mathrm{p}<0.0001)$

3. Degree of change in GMFM-66 with improvement $(\mathrm{p}<0.007)$

4. Degree of change in GMFM-66 age-adjusted centiles with improvement $(\mathrm{p}<0.005)$

The mean improvement in GMFM-66 and GMFM-66 ageadjusted centiles from pre-op to 24 months was 4.41 and 17.94 respectively. There were no complications requiring return to theatre.

CONCLUSION: SDR is safe and in combination with physiotherapy can improve motor function in well selected children with GMFCS level IV.

Keywords: Cerebral palsy, Selective Dorsal Rhizotomy

\section{PF-018}

\section{Functional}

Transcranial magnetic stimulation derived presurgical language mapping assists surgical planning and preserves language function in a predominately pediatric cohort undergoing epilepsy or brain tumor surgery

Talitha Boardman ${ }^{1}$, Savannah Gibbs ${ }^{2}$, James W Wheless ${ }^{1}$, Frederick A Boop ${ }^{3}$, Shalini Narayana ${ }^{1}$

${ }^{1}$ Department of Pediatrics, University of Tennessee Health Science Center, Memphis, TN; Neuroscience Institute, Le Bonheur Children's Hospital, Memphis, TN

${ }^{2}$ Neuroscience Institute, Le Bonheur Children's Hospital, Memphis, TN
${ }^{3}$ Department of Neurosurgery, University of Tennessee Health Science Center, Memphis, TN; Neuroscience Institute, Le Bonheur Children's Hospital, Memphis, TN

BACKGROUND AND AIM: To examine the efficacy of transcranial magnetic stimulation (TMS) for identifying critical language areas in patients undergoing epilepsy or brain tumor surgery.

METHODS: n 62 patients ( $5-64$ y, $48 \leq 18$ y, 37 males) who underwent presurgical TMS language mapping, we examined the relationship between excision of TMS-identified language locations and their immediate post-operative language function.

RESULTS: TMS identified language areas in all patients and facilitated surgical planning for resection of tumors or epileptogenic foci without resulting in any post-operative language deficits in 53 patients (85\%). Five of 34 patients in whom TMS locations were not resected had post-operative language deficits, likely due to the operated sites not being surveyed by TMS or surgical inclusion of underlying white matter tracts. Twenty-three patients in whom some TMS-identified language areas were removed experienced no language deficits. However, this was to be expected because TMS also localized additional language areas away from the lesion in the same hemisphere $(n=13)$ or in the contra-lesional hemisphere $(n=10)$. One patient had intact language despite having several TMS locations removed. Four patients in whom the lesional hemisphere was deemed language dominant by TMS, and in whom the critical language areas were localized within the lesion, exhibited post-operative language deficits. The calculated sensitivity of TMS for localizing critical language areas was 98\% and the accuracy was $90 \%$.

CONCLUSIONS: TMS successfully determined the dominant hemisphere for language and localized critical language areas in a predominantly pediatric cohort with epilepsy or brain tumor. TMS was especially useful in our cohort of children $\leq 10$ years, in whom cortical stimulation mapping is often unsuccessful. These data are the largest to show the utility of TMS in planning surgical resection and optimizing post-operative language outcome and suggest that TMS is a safe, non-invasive language mapping technique with high efficacy.

Keywords: brain tumor, epilepsy, transcranial magnetic stimulation, functional mapping, language mapping, presurgical

\section{PF-019}

\section{Germ Cell Tumors}


Optimization of intracranial germinoma treatment: A single institution experience with 213 patients supports radiotherapy alone with reduced volume and dose

Dong Seok Kim ${ }^{1}$, Seung Woo Park ${ }^{2}$, Eun Kyung Park ${ }^{1}$, Kyu Won Shim ${ }^{1}$, Chang Ok Suh ${ }^{3}$

${ }^{1}$ Department of Neurosurgery, Severance Children's Hospital, Yonsei University College of Medicine, Seoul, Korea ${ }^{2}$ Kangwon University College of Medicine, Chuncheon, Korea

${ }^{3}$ Department of Radiation Oncology, Bundang CHA Medical Center, CHA University, Bundang, Korea

OBJECTIVE: To investigate the optimal management regarding the radiotherapy (RT) target volume and a combination of RT and chemotherapy (CTx) of intracranial germinoma.

MATERIAL-METHODS: We retrospectively evaluated 213 patients with intracranial germinoma treated during 1976-2017. The treatments within our institute have changed according to diagnostic techniques and clinical experience. From 1978, stereotactic biopsy was performed. From 1984, trial RT and tumor marker study were performed and craniospinal irradiation (CSI) was conducted for presumed germinoma. RT volume was reduced for the patients with complete response after CTx was introduced in 1991. From 2011, institutional policy shifted to the 'RTonly approach' consisted of reduced dose and smaller target volume (whole ventricle [WV]/whole brain [WB] instead of CSI for localized disease). RT doses were gradually reduced to $36 \mathrm{~Gy}$ to the primary site and $18 \mathrm{~Gy}$ for CSI.

RESULTS: Median age at diagnosis was 16.2 years; 118 and 95 patients were pathologically proven and presumed germinoma, respectively; 151 and 62 patients had localized and disseminated disease, respectively. With a median follow-up of 141 months, the 10-year overall survival rates were $95.6 \%$. The recurrence rates were similar between RT-only (9/137, $6.6 \%)$ and RT + CTx $(4 / 73,5.5 \%)$, while all 3 patients who received CTx-only experienced recurrence. These were highest in local RT group $(10 / 29,34.5 \%)$, but were relatively low in WV/WBRT $(3 / 51,5.9 \%)$ and CSI group (0/130, $0 \%)$. In-field failure occurred in 3 patients. The causes of 14-deaths were progression $(n=4)$, 2nd malignancy $(n=4)$, CTx toxicity $(n=2)$, and others $(n=4)$. Among 33 patients treated with "RT-only approach" after 2011, 2 patients (6.1\%) who received WV/WBRT experienced recurrence at spinal cord and biopsy tract, respectively.

CONCLUSION: The additional benefit of CTx seems minimal in treating localized intracranial germinoma. The policy of RT-only approach with reduced RT target volume and dose seems reasonable.
Keywords: Germinoma, radiation therapy, chemotherapy, long-term follow-up, survival

\section{PF-020}

\section{Global Pediatric Neurosurgery}

One-stage high-density focal stereo-array SEEG-guided radiofrequency thermocoagulation for the treatment of pediatric giant hypothalamic hamartomas

Min Wang, Yuanfeng Zhou, Hao Li, Rui Zhao

Department of Neurosurgery, Children's Hospital of Fudan University, Shanghai, China

OBJECTIVE: BACKGROUND: Giant hypothalamic hamartomas $(\mathrm{HHs})$ are extremely rare lesions, for which the treatment is challenging. While minimally invasive treatments such as radiofrequency thermal coagulation and laser ablation have improved seizure outcomes, multiple operations are often required. This study investigated the value of onestage stereo-array radiofrequency thermocoagulation based on stereotactic electroencephalography (SEEG) for pediatric giant HHs.

MATERIAL-METHODS: We analyzed the clinical data of six patients with giant HHs (Delalande type IV or masses with a maximum diameter $>30 \mathrm{~mm}$ ) who underwent stereotactic electrode implantation between November 2017 and April 2019. After a multidisciplinary discussion, we designed a high-density focal stereo-array electrode implantation strategy. SEEG-guided bipolar coagulations were performed between two contiguous contacts of the same electrode, or between two adjacent contacts of different electrodes.

RESULTS: Among the six patients, three were male and three were female, with an average age of $5.08 \pm 4.73$ years (range 1.4-12 years); the average follow-up duration was $20.17 \pm 5.49$ months. One patient had previously undergone open surgery. Four patients had gelastic sezures, one had gelastic and tonic seizures, and one had gelastic and generalized tonic-clonic seizures. The number of implanted electrodes ranged from 3 to 7 , with an average of 5.33. One patient had transient diabetes insipidus after the operation, and no child had fever or new hormone metabolisms disorder after surgery. Four patients had Engel I classification outcomes, and two patients had Engel II classification outcomes.

CONCLUSION: One-stage high-density focal stereo-array SEEG-guided radiofrequency was safe and effective for treating pediatric giant $\mathrm{HH}$ patients. 
Keywords: children, stereoelectroencephalography, hypothalamic hamartoma, radiofrequency thermocoagulation, treatment

\section{PF-021}

\section{Global Pediatric Neurosurgery}

Long-term outcome of large sylvian arachnoid cysts: the role of surgery has been exaggerated

Kyung Hyun Kim ${ }^{1}$, Ji Yeoun Lee ${ }^{2}$, Ji Hoon Phi ${ }^{1}$, Seung Ki $\mathrm{Kim}^{1}$, Byung Kyu $\mathrm{Cho}^{3}$, Kyu Chang Wang ${ }^{1}$

${ }^{1}$ Division of Pediatric Neurosurgery, Seoul National University Children's Hospital, Seoul, Republic of Korea

${ }^{2}$ Department of Anatomy and Cell Biology, Seoul National University College of Medicine, Seoul, Republic of Korea ${ }^{3}$ Department of Neurosurgery, the Armed Forces Capital Hospital, Seongnam, Republic of Korea

OBJECTIVE: The surgical indications for some arachnoid cysts (ACs) are controversial. While surgical procedures can be effective when an $\mathrm{AC}$ is a definite cause of hydrocephalus or papilledema, most ACs do not cause any symptoms or signs. Some surgeons perform several procedures on ACs because of their large size. The purpose of this study was to compare the long-term outcomes of Galassi type III ACs between surgery and nonsurgery groups.

MATERIAL-METHODS: The medical records of sixty patients diagnosed with sylvian ACs (Galassi type III) who visited Seoul National University Children's Hospital from July 1990 to March 2018 were analyzed. We compared the outcome between two groups who were treated differently. RESULTS: Out of the 60 patients, 27 patients had no symptoms, 19 patients had vague symptoms and signs associated with ACs, and the remaining 14 patients had definite ACrelated symptoms and signs. Thirty-eight patients underwent surgery, and 22 patients underwent observation. Some operations were accompanied by complications. Among the 33 patients in the surgery group, excluding 5 with hydrocephalus or papilledema, 8 patients needed 18 additional operations. However, there were no patients in the nonsurgery group who needed surgical intervention during follow-up, although the size of the $\mathrm{AC}$ increased in 2 patients. Changes in $\mathrm{AC}$ size were not correlated with symptom relief.

CONCLUSION: When patients with hydrocephalus or papilledema were excluded, there was no difference in the outcomes between the surgery and nonsurgery groups regardless of the size of the sylvian AC. Surgeons should be cautious in deciding whether to operate.

Keywords: Arachnoid cyst, Children, Sylvian fissure, Surgery, Observation
PF-022

Global Pediatric Neurosurgery

Management of residual bone defects after craniosynostosis surgery

Motaz Alsereihi, Alexandru Szathmari, Djene Ibrahima Kaba, Rel Gerald Boukaka, Pierre Aurelien Beuriat, Federico Di Rocco, Carmine Mottolese

Mother Child Hospital, Civilian hospitals of Lyon, Claude Bernard University

OBJECTIVE: Surgical treatment of craniosynostosis in children may generate secondary cranial vault defects by incomplete ossification process. The incidence of cranial defects after craniosynostosis surgery is poorly reported in the literature with a variable incidence of $0,5 \%$ to $18,2 \%$. To better understand the impact and management of the cranial defects after craniosynostosis surgery, we selected and reviewed all cranioplasties that met this criterion between 2002 and 2019.

MATERIAL-METHODS: We found 41 patients $(28 \mathrm{M}$ and 13 F) operated between January 2002 to January 2019 who underwent cranioplasty for cranial defects secondary to craniosynostosis surgery in the infancy. All patient were at least 5 years old (range 5 to 17 years, median 11). The indication was aesthetic in 28 patients and for headaches, learning difficulties or changes in the cerebral blood flow in 13 patients. The material used in cranioplasty was the polymethyl-methacrylate (PMMA) in 40 patients and customized porous hydroxyapatite (CPHA) in 1 patient.

RESULTS: The mean follow-up period after cranioplasty was of 37 months (range 2 to 125 months, median of 27 months). In the postoperative period there were no mortality nor neurological complications. There were 3 cases (7\%) of superficial local postoperative wound infection but with no evidence of long-term infection. The long-term CT scan follow-up showed no material migration, dislocation or fractures.

CONCLUSION: Considering the characteristics of the cranial vault after craniosynostosis surgery the treatment by cranioplasty with heterologous materials appears to be feasible thus avoiding donor site morbidity. For selected cases with small defects, our experience shows that the use of PMMA may be a safe alternative for the cranial vault repair in children older than 5 years.

Keywords: Cranial vault defects after craniosynostosis surgery, Cranioplasty, poly-methyl-methacrylate (PMMA)

PF-023 
Global Pediatric Neurosurgery

Local venous and pericerebral cerebrospinal fluid changes in trigonocephaly

Garcia Gonzalez Oscar ${ }^{1}$, Di Rocco Federico ${ }^{1}$, Szathmari Alexandru ${ }^{1}$, Beuriat Pierre Aurélien ${ }^{2}$, Paulus Christian ${ }^{3}$, Gleizal Arnaud ${ }^{2}$, Mottolese Carmine ${ }^{1}$

${ }^{1}$ Pediatric Neurosurgery, Hôpital Femme Mère Enfant, Hospices Civils de Lyon, Université de Lyon, Lyon, France

${ }^{2}$ Centre de Référence Craniosténoses, Neurochirurgie Pédiatrique, Hôpital Femme Mère Enfant, Université de Lyon, 59 bd Pinel, 69,677 Bron Cedex, France

${ }^{3}$ Maxillo-Facial Surgery, Hôpital Femme Mère Enfant, Université de Lyon, Lyon, France

OBJECTIVE: Abnormal emissary veins (EV) and abnormal pericerabral effusions (PE) may be found in trigonocephaly suggesting an associated disturbances in CSF dynamics. However, the incidence of such findings and their significance have not been fully elucidated. The aims of this study are to clarify whether these phenomena identify specific subtypes of trigonocephaly by evaluating their relationship to morphology of the affected suture (" $\Omega$ ", "V" or Flat type) and its thickness on the grounds of computed tomographic (CT) scan findings. as well as the value of the interfrontal angle.

MATERIAL-METHODS: The preoperative brain CT scans of 74 children ( 52 boys, 22 girls) with trigonocephaly who had undergone fronto-orbital remodeling between 2007 and 2016 at The French Referral Center for Craniosynostosis of Lyon were evaluated, retrospectively. Several parameters were evaluated: the interfrontal angle, thickness and aspect of the fused suture, evidence of metopic emissary veins, volume of the pericebral CSF spaces.

RESULTS: Preoperatively, an endocranial metopic ridge was seen in $70 \%$ ( 52 children with a " $\Omega$ " or / "V" ridge). Emissary veins were identified in 34 of 74 patients (45\%), at a mean distance of $2.04 \mathrm{~cm}(1.18-2.94 \mathrm{~cm})$ from the nasion. The presence of EV was significantly correlated to the " $\Omega$ " type of metopic ridge $(\mathrm{p}<0.05)$. Among 50 infants who underwent the CT scan before 50 weeks of age, 17 (34\%) had larger PE than expected for their age. The presence of large PE significantly correlated with that of EV $(\mathrm{p}<0.05)$ and with the " $\Omega$ " type of ridge $(\mathrm{p}<0.05)$.

CONCLUSION: We can hypothesize an association between $E V, P E$ and the " $\Omega$ " notch. The presence of emissary veins could be an indicator of local venous hypertension due to the sagittal sinus constriction. The long-term results of distinguishing between children with normal peripheral spaces and pathological PE are still to be determined.

Keywords: trigonocephaly, cerebrospinal fluid, changes
PF-024

\section{Global Pediatric Neurosurgery \\ Pediatric Neurosurgery in Asia and Australasia: Train- ing and Clinical Practice}

Ronnie E. Baticulon ${ }^{1}$, Michael C. Dewan ${ }^{2}$, Nunthasiri Wittayanakorn $^{3}$, Philipp R. Aldana ${ }^{4}$, Wirginia J. Maixner ${ }^{5}$

${ }^{1}$ Division of Neurosurgery, Department of Neurosciences, University of the Philippines - Philippine General Hospital, Manila, Philippines

${ }^{2}$ Division of Neurosurgery, Hospital for Sick Children, Toronto, Ontario, Canada

${ }^{3}$ Division of Neurosurgery, Department of Surgery, Queen Sirikit National Institute of Child Health, Bangkok, Thailand ${ }^{4}$ Division of Pediatric Neurosurgery, University of Florida Health Jacksonville, Jacksonville, Florida, USA

${ }^{5}$ Department of Neurosurgery, The Royal Children's Hospital, Melbourne, Australia

OBJECTIVE: There is limited data on the pediatric neurosurgical workforce in Asia and Australasia. The training and clinical practice of pediatric neurosurgeons need to be characterized in order to identify gaps in knowledge and skills, thereby establishing a framework from which to elevate pediatric neurosurgical care in the region.

MATERIAL-METHODS: An online survey for pediatric neurosurgeons was created in REDCap, collecting demographic information and data on pediatric neurosurgical training and clinical practice. The link to answer the survey was sent to the mailing lists of the Asian Australasian Society for Pediatric Neurosurgery and Japanese Society for Pediatric Neurosurgery, and spread through social media. The survey was open to neurosurgeons who operated on patients $\leq 18$ years old in Asian Australasian countries. Data were stratified and compared based on surgeon training and World Bank income group.

RESULTS: A total of 155 valid responses were analyzed, representing neurosurgeons from 21 countries. 107 considered themselves pediatric neurosurgeons, of whom $66 \mathrm{com}$ pleted pediatric neurosurgery training. Neurosurgeons in East Asia commonly undergo fellowship in their home countries while the rest train mostly in North America, Europe, and Australia. A majority had operating privileges, and subspecialty pediatric training usually lasted from 6 months to 2 years. On average, trained pediatric neurosurgeons perform a higher number of pediatric neurosurgical operations/year compared with non-pediatric trained respondents $(131+129$ vs. $56+64, p=0.0001)$. Mean number of total neurosurgical operations/year is similar for both groups $(184+129$ vs. $178+142, \mathrm{p}=0.80)$. Respondents expressed desire to train 
further in pediatric epilepsy, spasticity, vascular malformations, craniofacial disorders, and brain tumors.

CONCLUSION: Both pediatric and general neurosurgeons provide neurosurgical care to children in Asia and Australasia. Skill sets and training needs in pediatric neurosurgery vary, depending on the country's economic status, and between pediatric trained and non-pediatric trained surgeons.

Keywords: pediatric neurosurgery fellowship, neurosurgical education, global neurosurgery, Asia Australasia

\section{PF-025}

\section{Global Pediatric Neurosurgery Folate Fortification and Supplementation in Prevention of Neural Tube Defects: A Systematic Review of Policy}

Nathan A. Shlobin ${ }^{1}$, Melissa A. LoPresti ${ }^{2}$, Rebecca Y. Du ${ }^{2}$, Sandi K. Lam ${ }^{1}$

${ }^{1}$ Department of Neurological Surgery, Feinberg School of Medicine, Chicago, USA; Division of Pediatric Neurosurgery, Lurie Children's Hospital, Chicago, USA

${ }^{2}$ Department of Neurosurgery, Baylor College of Medicine, Houston, USA; Division of Neurosurgery, Texas Children's Hospital, Houston, USA

OBJECTIVE: Neural tube defects (NTDs) are common, resulting in mortality, morbidity, and impaired quality of life. Sweeping public health policy to increase folate consumption among women who are or plan to become pregnant lags behind scientific evidence of its effectiveness in reducing NTDs. We aimed to identify the types of policies applied, associated outcomes, and impact of folate fortification and supplementation on NTDs worldwide. By identifying the most effective legislation, we aim to focus advocacy efforts to more broadly effect change in neurosurgery.

MATERIAL-METHODS: A systematic review was conducted exploring folate fortification and supplementation policies using PubMed and Scopus databases. Titles and abstracts from all articles identified were read and selected for full-text review. Studies meeting inclusion criteria were reviewed in full and analyzed for study design, aim, population, interventions, and outcomes.

RESULTS: Of 1,637 resultant articles, 54 full-text articles were included. Policies from 17 countries were evaluated, of which nine $(52.9 \%)$ were high income countries, seven $(41.2 \%)$ were upper-middle income countries, and one $(5.9 \%)$ were lower-middle income countries. Mandatory folate fortification was effective at reducing rates of NTDs. Fortification also resulted in decreased hospitalization rates and deaths after discharge and increased first-year survival for infants with NTDs. Folate supplementation resulted in decreased NTD prevalence. Folate fortification or supplementation resulted in decreased NTD prevalence. Reductions in rates of NTDs were associated with significant cost savings.

CONCLUSION: Mandatory folate fortification and recommended supplementation policies are effective in decreasing NTD rates when applied. A comprehensive approach incorporating mandatory folate fortification, appropriate folate supplementation, and improved infrastructure and access to prenatal care, if more broadly adopted, would likely lead to decreased incidence of NTDs worldwide. This approach would be context-specific, accounting for regional access to healthcare and social determinants of health, and emphasizing education. As caretakers of children with NTDs, neurosurgeons should advocate for comprehensive policy action.

Keywords: folate, folic acid, spina bifida, neural tube defects, fortification, supplementation

\section{PF-026}

\section{Global Pediatric Neurosurgery \\ Accuracy of identifying pediatric craniofacial abnormal- ities requiring surgical intervention through the assess- ment of caregiver provided photographs}

Alexander Cheong, Ash Singhal, Patrick Mcdonald, Mandeep Tamber

Division of Neurosurgery, Department of Surgery, British Columbia Children's Hospital, University of British Columbia, Vancouver, BC, Canada

OBJECTIVE: Assessment of head shape is a common reason for referrals to pediatric neurosurgery clinics. To streamline patient assessments, we investigated whether evaluation of standardized screening photographs were of value in triaging referrals.

MATERIAL-METHODS: Parents of children referred to our neurosurgery clinic for assessment of head shape submitted a series of standardized photographs which were blindly evaluated by 7 independent raters, including 3 neurosurgeons, 2 plastic surgeons, a pediatrician, and an OT. Each rater documented their diagnosis (presence or absence of potentially surgically actionable craniosynostosis) and their level of confidence in their diagnosis. All children were subsequently evaluated in person by an experienced pediatric neurosurgeon, and this diagnosis was used as the gold standard.

RESULTS: Of the 38 children evaluated, 11 (28.9\%) had craniosynostosis diagnosed on an in-person assessment. Fleiss-kappa amongst all 7 raters was $0.67(0.58,0.76)$, indicating substantial agreement related to the diagnosis of craniosynostosis based on photographic assessments. 
Individual raters' diagnostic performance based on photographic evaluation (sensitivity range $0.8-1.0$; specificity range $0.74-0.88$; PPV range $0.65-0.81$; NPV range 0.89-1.0) compared favourably with the performance of the "majority rule" photographic diagnosis of all 7 raters combined [sensitivity $=1.0(0.71,1.0)$; specificity $=0.88$ $(0.71,0.98)$; PPV $=0.82(0.61,0.93)$; and $\mathrm{NPV}=1.0]$, the 5 craniofacial surgeons [sensitivity $=1.0(0.72,1.0)$; specificity $=0.92(0.75,0.99) ; \mathrm{PPV}=0.87(0.63,0.96)$; and $\mathrm{NPV}=1.0$ ] or the 3 neurosurgeons [sensitivity $=1.0(0.69$, $1.0)$; specificity $=0.85(0.66,0.96) ; \mathrm{PPV}=0.77(0.58,0.89)$; and $\mathrm{NPV}=1.0]$.

CONCLUSION: Based on the evaluation of standardized photographs, experienced craniofacial practitioners were able to accurately identify patients with craniosynostosis who may require surgical intervention as well as those whose head shape abnormality would not require surgery. Individual trained raters appear to perform as well as group consensus. Photographic pre-screening may be useful for discriminating between craniosynostosis and plagiocephaly, the two most common etiologies of an abnormal head shape.

Keywords: Craniosynostosis, diagnosis, photographs, accuracy, screening

\section{PF-027}

\section{Global Pediatric Neurosurgery}

Chiari Type 1 Malformation Surgery In Children: French Multicentric 10-Year Case Series

Edouard Mazerand ${ }^{1}$, Sandro Benichi ${ }^{2}$, Giovanna Paternoster $^{2}$, Alice Rolland ${ }^{3}$, Pierre Antherieu ${ }^{4}$, Julien Todeschi ${ }^{5}$, Lawrence Kamdem Noumoye ${ }^{6}$, Vianney Gilard ${ }^{7}$, Maxime Bretonnier ${ }^{8}$, Luc Le Fournier ${ }^{1}$, Vincent Jecko ${ }^{6}$, Edouard Gimbert ${ }^{6}$, François Proust ${ }^{5}$, Sergio Boetto ${ }^{4}$, Thomas Roujeau ${ }^{3}$, Syril James ${ }^{2}$, Laurent Riffaud ${ }^{8}$, Matthieu Delion $^{1}$, Michel Zerah ${ }^{2}$, Didier Scavarda ${ }^{9}$

${ }^{1}$ Department of Neurosurgery, Angers University Hospital, Angers, France

${ }^{2}$ Department of Pediatric Neurosurgery, Hôpital NeckerEnfants Malades, Paris, France

${ }^{3}$ Department of Neurosurgery, Montpellier University Hospital, Montpellier, France

${ }^{4}$ Department of Neurosurgery, Toulouse University Hospital, Toulouse, France

${ }^{5}$ Department of Neurosurgery, Strasbourg University Hospital, Strasbourg, France

${ }^{6}$ Department of Neurosurgery, Bordeaux University Hospital, Bordeaux, France

${ }^{7}$ Department of Neurosurgery, Rouen University Hospital, Rouen, France
${ }^{8}$ Department of Neurosurgery, Rennes University Hospital, Rennes, France

${ }^{9}$ Department of Pediatric Neurosurgery, Marseille University Hospital, Marseille, France

BACKGROUND AND AIM: Chiari type 1 malformation (CM-1) is frequent in children and remains a surgical challenge. Several techniques have been described for posterior fossa decompression. No decision algorithm has been validated, and strategies are highly variable between institutions. Our objective was to define therapeutic guidelines considering each patient's specificities.

METHODS: We retrospectively collected data from children under 18-years-old, diagnosed with CM-1, treated surgically between 2008 and 2018 in eight French pediatric neurosurgical centers. Clinical features, morphological parameters and surgical techniques were collected. Clinical outcomes at 3 months, 1-2 years, 3-5 years and over 5 years were assessed by the Chicago Chiari Outcome Scale (CCOS). We performed a principal component analysis (PCA) to define patients' subgroups, and compared outcomes between these groups.

RESULTS: Data from 255 patients were collected. Mean age at surgery was $9.6 \pm 5.0$ years, syringomyelia was associated in $60.2 \%$ patients, dura was opened in $65.0 \%$ patients and $17.3 \%$ patients underwent a redo surgery for additional treatment. Mean CCOS was $14.4 \pm 1.5$ at 3 months, $14.6 \pm 1.9$ at $1-2$ years, $14.5 \pm 2.0$ at $3-5$ years and $14.3 \pm 1.9$ over 5 years. PCA identified five subgroups with different mechanisms underlying tonsillar herniation: constitutional bony anomaly, instable basilar invagination, arachnoiditis, atlantoaxial instability and stable ventral brainstem compression. Each subgroup matched with a specific surgical procedure. CONCLUSIONS: This French multicentric retrospective study allowed to identify five subgroups among pediatric patients who underwent surgery for CM-1, with different outcomes after surgery. Using this objective classification will help optimizing the results of surgery for these patients.

Keywords: Chiari 1 malformation; syringomyelia; posterior fossa decompression; duraplasty; tonsillar herniation; case series

\section{PF-028}

\section{Global Pediatric Neurosurgery The Paediatric Vault Score (PVS)}

Adam Shathur, Anusha Adeline Hennedige, Christopher Parks, Ajay Sinha, Jonathan Ellenbogen, William Dawes, David Richardson, Christian Duncan 
Paediatric Craniofacial Deformity, Alder Hey Children's Hospital, Eaton Road, Liverpool L12 2AP

BACKGROUND AND AIM: Paediatric Craniofacial Surgery encompasses the management of complex craniofacial anomalies ranging from simple single suture craniosynostosis to complex syndromic multi-suture craniosynostosis. As one of 4 supra-regional Craniofacial Centres in England, we operate on a high-volume of paediatric craniofacial patients. In light of the COVID-19 pandemic, access to theatres and numbers of operating lists were reduced, extending surgical waiting lists. A risk stratification tool was thus devised to prioritise patients.

METHODS: A Paediatric Vault Score (PVS) was devised based on parameters such as suture involvement and evidence of raised intracranial pressure. Each parameter was assigned a score which placed each patient into different risk categories. Validity of the scoring system was carried out by randomly selecting ten patients on the waiting list. Each patient's clinical information was summarised and presented in a uniform anonymised format. Six craniofacial consultants (4 neurosurgeons, 1 maxillofacial and 1 plastic surgeon) were selected to perform the ranking. The consultants independently analysed and ranked patients from $1-10$, (patient 1 being the highest priority). Each scorer's ranking was verified against the PVS template. Two cycles of the scoring were carried out one month apart.

RESULTS: Kendall Rank correlation coefficient was used to analyse the results. Analysis revealed all 6 clinicians to be concordant, with a mean Kendall Rank score of 0.61.

CONCLUSIONS: The PVS system is the first vault scoring system devised to aid in risk stratification and in waiting list prioritisation. The impetus to develop this has come about due to the constraints from the impact of the COVID impact. However, its use has far-reaching benefits and forms the basis of decision-making for treating patients with craniosynostosis. The PVS has been validated as an efficient and reliable tool to rank patients according to their need for surgery and to aid the surgeon in decision-making for craniosynostosis patients.

Keywords: Craniofacial deformity, scoring system, oral and maxillofacial, plastic, neurosurgery, paediatric

\section{PF-029}

\section{Hydrocephalus}

Reliability of the Radiopharmaceutical Shunt Flow Study in the Presence of Stable Ventricular Size for the Detection of a Cerebrospinal Fluid Shunt Malfunction

Jeffrey John Quezada ${ }^{1}$, J. Gordon Mccomb $^{2}$
${ }^{1}$ Division of Neurosurgery, Children's Hospital Los Angeles, Los Angeles-CA, USA

${ }^{2}$ Division of Neurosurgery, Children's Hospital Los Angeles, Los Angeles-CA, USA; Department of Neurological Surgery, Keck School of Medicine, University of Southern California, Los Angeles-CA, USA

OBJECTIVE: Evaluate the reliability of a radiopharmaceutical (RP) shunt flow study in the presence of stable ventricular size for detection of cerebrospinal (CSF) shunt malfunction.

MATERIAL-METHODS: All shunt flow studies done in 2014-2018 were identified. Included were only patients in whom a MRI/CT scan was done during admission for shunt malfunction and showed no increase in ventricular size when compared with the most recent prior MRI/CT scan when the patient was asymptomatic. Exams consisted of reservoir tapping and injecting Tc-99 m DTPH per protocol. Rapid reflux of tracer into the ventricles followed by rapid flow from the distal end of the shunt was considered a normal study. No ventricular reflux was considered a proximal malfunction while slow, no distal runoff, or tracer extravasation along the shunt tract was deemed a distal obstruction.

RESULTS: 142 RP flow studies in 112 patients met the above criteria. Operative intervention was not undertaken in $102(91 \%)$ of the 112 normal studies. Of the $10(9 \%)$ operated patients 8 had a proximal obstruction, 1 distal and 1 no obstruction. Of the 30 abnormal flow studies $9(30 \%)$ patients were not operated on as their symptoms subsided. Of the $21(70 \%)$ operated patients obstruction was proximal in 9, distal in 5 and for 7 tubing was fractured/disconnected. Regression analysis indicated a significant association between exam interpretation and the odds for revision $(\mathrm{OR}=27,95 \% \mathrm{CI}=10-75, \mathrm{p}<0.0001)$. No other clinical variables were significant. Shunt flow study alone compared to shunt flow study plus MRI/CT did not change the sensitivity (70\%/70\%), but did increase specificity from 92 to $100 \%$ and accuracy from 87 to $94 \%$.

CONCLUSION: RP shunt flow studies in patients with symptoms of shunt malfunction in whom no change in ventricular size occurred when compared to being asymptomatic was of definite value in deciding as to operatively intervene.

Keywords: radiopharmaceutical, shunt flow study, shunt malfunction, hydrocephalus, cerebrospinal fluid, ventricular size

PF-030

Hydrocephalus

Management of multiloculated hydrocephalus with emphasis on role of $\mathrm{CT}$ ventriculography 
Siddharth Vankipuram $^{1}$, Chhitij Srivastava ${ }^{2}$, Bal Krishna Ojha $^{2}$, Anil Chandra ${ }^{2}$, Sunil K Singh ${ }^{2}$, Somil Jaiswal ${ }^{2}$, Manish Jaiswal ${ }^{2}$

${ }^{1}$ Department of Pediatric Neurosurgery, Children's Surgical Hospital, Aurangabad, India

${ }^{2}$ Department of Neurosurgery, King George's Medical University, Lucknow, India

OBJECTIVE: We performed a retrospective review of children diagnosed with multiloculated hydrocephalus (MLH) in our institute. The goal was to analyze the different diagnostic and therapeutic modalities used with special emphasis on CT ventriculography (CTV).

MATERIAL-METHODS: Male and female patients below the age of 18 years diagnosed with MLH were included. Cases of uniloculated hydrocephalus like entrapped temporal horn or isolated fourth ventricle were excluded. We used Iohexol for CTV and Gadodiamide for MR ventriculography. Neuroendoscopic procedures performed were endoscopic fenestration, endoscopic third ventriculostomy (ETV), endoscopic septostomy, endoscopic aqueductoplasty or a combination of the above. The cohort was divided into two groups (endoscopic or shunt) based on initial surgical intervention. RESULTS: A total of 52 patients were included, with 43 boys and 9 girls. The average age of presentation was 7.7 months. The most common predisposing factor for MLH was neonatal meningitis seen in 30 patients. Mean duration of follow up was 39 months. CTV was used in 26 patients and MR ventriculography in three patients. In one patient, the diagnosis of MLH was ruled out after ventriculography. Patients who underwent ETV only had the best outcome with $71.4 \%$ success rate. At the end of follow up, 14 patients (27\%) were shunt independent.

CONCLUSION: The present study indicates that CTV helps to accurately define the anatomy of the ventricles and determine the site of physiological CSF obstruction. This helps in therapeutic planning and in avoiding misdiagnoses. Further, neuro-endoscopy has the potential to lead to shunt independence in some patients.

Keywords: Neuroendoscopy, endoscopic fenestration, multiseptate hyrdrocephalus, CT ventriculogrpahy, post-infective hydrocephalus

\section{PF-031}

\section{Hydrocephalus}

Efficacy and safety of intraventricular fibrinolytic therapy for post intraventricular hemorrhagic hydrocephalus in extreme low birth infants: A preliminary clinical study

Young Soo Park, Tae Kyun Kim, Hiroyuki Nakase
Department of Neurosurgery and Children's Medical Center, Nara Medical University, Kashihara, JAPAN

OBJECTIVE: Management of post IVH hydrocephalus (PIVHH) in low-birth-weight-infants (LBWls) is challenging and controversial. We devised fibrinolytic therapeutic strategy attempted to remove out bloody CSF via external ventricular drainage combined with urokinase injection into lateral ventricle, called Ventricular Lavage (VL) therapy from early stage of disease. Aim of this study is to evaluate efficacy and safety of our unique therapy.

MATERIAL-METHODS: In total 43 consecutive infants with PIVHH (grade 3: 16, grade 4: 27) were analyzed. The majority were extremely LBWIs, with 39 cases. The VL therapy was consisted of two concepts: first was continuous external ventricular drainage (EVD) management using a very fine catheter and second was intermittent slow injection $6000 \mathrm{IU}$ of urokinase every 3 to $6 \mathrm{~h}$ to dissolve hematomas. The duration of fibrinolytic therapy was limited to a maximum of 14 days. The endpoints included the requirement for V-P shunt, treatment-related complications, and functional outcomes.

RESULTS: Early EVD management was performed in 25, and VL therapy was combined in 21. Five infants received late EVD management and the remaining 13 were treated conservatively. Fifteen of eighteen (83\%) LBWls received VL therapy did not require permanent shunt surgery. And there were no serious associated complications including secondary hemorrhage and infection. Two-thirds of infants treated in late stages required permanent shunt placement, and various shunt related complications occurred frequently. We defined neurological outcomes were good, if self-walking, verbal communication and self-feeding were satisfactory at 36 months. Early treatment group was significantly better (76\% vs. $40 \%$ ) despite the majority of IVH grade 4 .

CONCLUSION: Permanent shunt surgery was dramatically reduced in extremely LBWIs treated with VL therapy. In addition, the functional outcomes were also better than historical reports. Controlling sustained ICP, maintaining brain morphology, and actively dissolving hematomas could reduce white matter damage. Our VL therapy might be safe and promising strategy.

Keywords: PIVHH, pretem infants, low-birht-weight-ifants, EVD, fibrinolytic therapy, urokinase

PF-032

\section{Hydrocephalus}

Predictive value of immediate postoperative imaging for long-term success of endoscopic third ventriculostomy: a single-center experience 
Michelle W McQuinn, MD, Eveline Teresa Hidalgo, MD MSCI, Andrew Brunswick, MD, Conor Grady, MD, Harold Westley Phillips, MD, Nathan B Han, MD, David H Harter, MD

Division of Pediatric Neurosurgery, Department of Neurosurgery, Hassenfeld Children's Hospital at New York University Langone Health, New York City, USA

OBJECTIVE: Endoscopic third ventriculostomy (ETV) is a treatment option for obstructive hydrocephalus. It is common practice to obtain immediate postoperative magnetic resonance imaging (MRI) following ETV. This study investigated the utility of this practice by assessing the relationship between specific radiological findings and ETV outcome.

MATERIAL-METHODS: Retrospective chart review was performed for all patients who underwent their first ETV at NYULH from 1995 to 2017 . Patients with pre- and postoperative MRI and at least one year of follow-up were included. Radiographic features studied included: change in subarachnoid space, change in maximal diameter of the third ventricle (TV), change in bowing of the TV floor, and presence of postoperative flow void. Univariate and multivariate logistic regressions were performed to identify associations between the variables and ETV failure (any hydrocephalusrelated return to the operating room). Failed cases were subdivided based on time to failure (within 30 days, within six months, within one year). Sub-group analysis was performed by stratifying cohorts by age ( $>10$ years) and hydrocephalus etiology (obstructive).

RESULTS: Fifty-eight subjects were included (median age: 19 years; range 10 days -81 years). Nineteen $(32.8 \%)$ subjects failed within one year. Obstructive hydrocephalus was significantly associated with success at one year post-ETV. In the adult sub-group, etiology was a significant predictor of success at six and at twelve months. Individually, none of the radiological variables predicted success at any of the defined time points. The presence of any postoperative radiological change was significantly associated with success at six and at twelve months.

CONCLUSION: Individual radiological variables - including the presence of a flow void - were not significantly predictive of ETV outcome. However, the presence of any radiological change was significantly associated with a successful outcome. Therefore, careful consideration is needed to determine the clinical value of immediate post-operative imaging following ETV.

Keywords: Endoscopic third ventriculostomy, Postoperative imaging, Long-term success, Obstructive hydrocephalus, Radiologic findings

PF-033
Hydrocephalus

ShuntScope in Children- Experience with 61 Procedures

Anna Prajsnar Borak, Joachim Oertel

Department of Neurosurgery, University Hospital of Saarland, Homburg, Germany

OBJECTIVE: Endoscopic procedures for treatment of pediatric hydrocephalus have become nowadays a well-established technique for most of pediatric neurosurgeons. The implementation of ShuntScope, in simplification of management using shunting technology, has gained increasing acceptance. The value of the ShuntScope, offered in treatment of pediatric hydrocephalus is discussed in this presentation. Particular attention is given to indications, surgical technique, success rate, results and experiences gained in our institution. Illustrative cases are selected and described in detail.

MATERIAL-METHODS: Between February 2012 and April 2020, a ShuntScope was applied in 61 procedures in 41 children (27 males, 14 females, age range 2 months- 18 years). Indications for surgery included transaqueductal stent placement (7), first time shunting (10), burr hole reservoir insertion (8), shunt revision surgery of the central catheter (26), transforaminal ventricular catheter (VC) placement after foraminoplasty (1), septum pellucidotomy with transseptal VC placement (4) and transcystic VC placement (4), ShuntScope- assisted Rickham-reservoir explanation (1).

RESULTS: In two cases of aqueductal stenting, because of unmanageable anatomical circumstances, the intended therapy strategy had to be abandoned and switched to VC placement into lateral ventricle. Intraventricular limited image quality, apart from three cases, was absolutely sufficient for anatomical orientation. Due to catheter covering of the endoscope, no parenchymal damage or bleeding occurred.

CONCLUSION: Intra-catheter endoscope is a safe and in most of cases efficient technique. Whether performed alone or in combination with other endoscopic procedures, the application of the ShuntScope should be considered in treatment of pediatric hydrocephalus.

Keywords: ShuntScope, Hydrocephalus, Endoscopy

\section{PF-034}

Hydrocephalus

Complement Activation Leads to Worse Cognitive Outcomes Following Germinal Matrix Hemorrhage in a Chronic Mouse Model

Mohammed Alshareef $^{1}$, Ramin Eskandari ${ }^{1}$, Stephen Tomlinson $^{2}$ 
${ }^{1}$ Department of Neurosurgery, Medical University of South Carolina, Charleston, SC, USA

${ }^{2}$ Department of Immunology and Microbiology, Medical University of South Carolina, Charleston, SC, USA

BACKGROUND AND AIM: Germinal matrix hemorrhage (GMH) is a common neonatal neurologic pathology that results in sequalae such as post-hemorrhagic hydrocephalus (PHH) and periventricular leukomalacia (PVL), likely secondary to post-hemorrhagic scarring and gliosis. The complement cascade has been established as a major player in secondary injury and subsequent development of gliosis. Here, we discuss the progressive cognitive and motor decline in animals following GMH and the protective role of a novel complement inhibitor, CR2Crry.

METHODS: A neonatal mouse GMH model was created by injection of collagenase into the subventricular zone of postnatal day four (P4) pups. A control (injured) group was compared to animals treated with a complement inhibitor (CR2Crry). Neurocognitive motor function and survival analysis was obtained on all animals up to 90 days of life. Histologic comparisons were performed at various time points up to 90 days, with a focus on inflammation and neuroprotection. RESULTS: A significant reduction in proportion of highgrade infarcts, bilateral ventricular involvement, and $\mathrm{PHH}$ (grade 5) was observed in CR2Crry-treated mice compared to control at the various time points up until 90 days. MR imaging was used at various time points (every 30 days) until 90 days, and revealed significant brain tissue sparing in animals treated with CR2Crry compared to controls. Barnes maze testing (behavioral) and CATWalk tests (motor) showed a higher rate of functional preservation in the treated animals. Histologic analysis of tissue showed reduced overall scar formation and inflammation in the treated animals. CONCLUSIONS: There are currently no treatments for GMH targeting the inflammatory pathway leading to gliosis and PVL. A better understanding of the inflammatory pathway associated is essential for the development of a treatment to reduce the effects of the secondary injury. Our results show that complement activation is detrimental to brain development, CSF absorption, and functional development in a chronic GMH model.

Keywords: Hydrocephalus, Germinal matrix hemorrhage, pediatrics, immunotherapy, Complement

\section{PF-035}

\section{Hydrocephalus}

The impact of shunting and endoscopic third ventriculostomy (ETV) on long-term ventricular and cranial size in infants with aqueductal stenosis
Ian Coulter ${ }^{1}$, Abhaya Kulkarni ${ }^{2}$, Spyros Sgouros ${ }^{3}$, Shlomi Constantini $^{4}$

${ }^{1}$ Department of Neurosurgery, Royal Victoria Infirmary, Newcastle upon Tyne, United Kingdom

${ }^{2}$ The Hospital for Sick Children, University of Toronto, Toronto, Ontario, Canada

${ }^{3}$ University of Athens Medical School and Department of Pediatric Neurosurgery, Mitera Children's Hospital, Athens, Greece

${ }^{4}$ Department of Pediatric Neurosurgery, Dana Children's Hospital, Tel Aviv Sourasky Medical Center, Tel Aviv University, Tel Aviv, Israel

BACKGROUND AND AIM: Measurements of head circumference (HC) and ventricular size are often collated together with other clinical and radiological parameters to establish the efficacy of intervention for infants with hydrocephalus. We aimed to assess the impact of shunting and endoscopic third ventriculostomy (ETV) on ventricular and $\mathrm{HC}$ measurements during the long-term follow-up of infants with aqueductal stenosis (AS).

METHODS: Data from the International Infant Hydrocephalus Study (IIHS) - a prospective, multicentre study of infants ( $<24$ months) with hydrocephalus secondary to AS treated with an ETV or shunt were analysed. Metrics assessed during the 5-year study period included: HC, HC centile, HC Z-score and frontal-occipital horn ratio (FOHR). Data were compared in an analysis of covariance, adjusting for baseline variables including age and sex.

RESULTS: One hundred and fifty-eight patients were analysed, 115 underwent an ETV, whilst 43 received a shunt as primary treatment. Both procedures led to improvements in the mean $\mathrm{HC}$ centile and $\mathrm{HC} \mathrm{z}$-score, a trend which continued until the fifth year of assessment. A similar trend was noted for FOHR which was measured 12 months and 3 years following surgery. Although the values were consistently higher for ETV compared to shunt, the differences in HC value, $\mathrm{HC}$ centile and $\mathrm{HC}$ z-score were not significant. ETV was associated with a significantly higher mean FOHR compared to shunting, when assessed at 12 months ( 0.52 vs 0.44 ; $\mathrm{p}=0.002)$ and 3 years $(0.46$ vs $0.38 ; \mathrm{p}=0.03)$ of follow-up. CONCLUSIONS: ETV and shunting both led to improvements in HC and FOHR during the long-term follow-up of infants with hydrocephalus secondary to AS. HC was not significantly different between the treatment modalities during a follow-up period of 5 years however, FOHR was greater in infants undergoing ETV when measured 1 and 3 -years following treatment.

Keywords: Hydrocephalus, Aqueductal Stenosis, ETV, Shunting 


\section{PF-036}

\section{Infection}

Risk factors of shunt-dependent hydrocephalus after purulent meningitis in children

\section{Yi Zhang, Wei Shi, Rui Zhao, Hao Li}

Department of Neurosurgery, Children's Hospital of Fudan University, ShangHai, China

OBJECTIVE: To analyze the related risk factors of shuntdependent hydrocephalus after purulent meningitis in children, find the predictive indicators of disease outcome, and provide clinical theoretical basis for disease diagnosis and treatment strategies.

MATERIAL-METHODS: A retrospective analysis of the cases of external ventricular drainage(EVD) for Purulent Meningitis in our hospital from January 2013 to December 2017 was performed. The patients who underwent permanent shunt were defined as the positive group, and those who removed the EVD catheter were the control group. Based on the analysis of clinical records, the risk factors of shuntdependent hydrocephalus were summarized.

RESULTS: A total of 48 cases were enrolled. The gender ratio was 1.2 , the median age at surgery was $0.3 \mathrm{Y}$, the median gestational week was 38 weeks, and the median weight was $6.0 \mathrm{~kg}$. Thirty-one cases $(64.5 \%)$ underwent permanent shunting. Statistical analysis showed that the basic clinical characteristics were similar between the two groups (age, gender, weight, gestational week). There was no difference in frontal occipital angle ratio baseline $(0.56 \pm 0.01$ VS $0.53 \pm 0.01 \mathrm{P}=0.72$ ), and the FO horn ratio in the control group decreased significantly after 7-10 days of EVD $(0.57 \pm 0.01$ VS $0.48 \pm 0.01 \mathrm{P}=0.001)$. ROC analysis indicated that FO horn ratio $\geq 0.52$ has predictive significance for permanent shunting, the area under the ROC curve $=0.818, \mathrm{P}=0.000$.

CONCLUSION: Our study found that the incidence of shunt-dependent hydrocephalus after purulent meningitis was higher(64.5\%). The reduction of forehead occipital angle ratio 7-10 days after EVD indicates good outcome, and the forehead occipital angle ratio of $\geq 0.52$ on 7-10 days after EVD has diagnostic and predictive significance for shunt-dependent hydrocephalus. The age of onset, premature birth history, different external drainage schemes, daily cerebrospinal fluid drainage, and infection severity did not play a role in predicting shunt-dependent hydrocephalus.

Keywords: shunt-dependent hydrocephalus; purulent meningitis; FO horn ratio

\section{Infection}

\section{Cerebellitis as a Neurosurgical Disease in Pediatrics}

Eric Andress Goethe ${ }^{1}$, Melissa Lopresti ${ }^{1}$, Brandon Bertot $^{1}$, Sandi Lam ${ }^{2}$

${ }^{1}$ Department of Neurosurgery, Baylor College of Medicine, Houston, Texas, USA

${ }^{2}$ Department of Neurosurgery, Northwestern University, Chicago, Illinois, USA; Division of Pediatric Neurosurgery at Lurie Children's Hospital, Chicago, Illinois, USA

OBJECTIVE: Cerebellitis is a rare condition involving cerebellar edema and dysfunction leading to cerebellar abnormalities, obstructive hydrocephalus, and elevated intracranial pressure. Literature regarding diagnostic evaluation and the role of neurosurgery in management is incomplete. We explore the role of neurosurgical intervention in pediatric cerebellitis, highlighting the diagnostic work up and treatments applied.

MATERIAL-METHODS: A retrospective review was conducted of all pediatric patients diagnosed with cerebellitis at a single center from June 2008 to February 2019. Clinical, laboratory, radiological, treatment, and follow-up data were analyzed.

RESULTS: Nine patients, four males $(44.4 \%)$ and five females (55.6\%) across varied racial and ethnic backgrounds, were identified. Common presenting symptoms were headache $(n=6,66.7 \%)$, emesis $(n=5,55.6 \%)$, and altered mental status $(n=4,44.4 \%)$. Six $(66.7 \%)$ had associated infections. Imaging abnormalities included tonsillar ectopia $(\mathrm{n}=8,88.9 \%)$, bilateral cerebellar T2 hyperintensity $(n=6,66.7 \%)$, and obstructive hydrocephalus $(n=6$, $66.7 \%$ ). Unilateral $\mathrm{T} 2$ hyperintensity was observed in one patient $(11.1 \%)$. Management included antibiotics, antivirals, corticosteroids, mannitol, and hypertonic saline. Neurosurgical consultation was requested for all patients $(n=9,100 \%)$. Four (44.4\%) required external ventricular drain (EVD) placement for a mean 11 days (SD 6.8, range 4-20) for hydrocephalus; none required surgical decompression, permanent shunting, or additional neurosurgical interventions. Seven patients (77.8\%) required ICU care for a mean 11.7 days (SD 14.0 range 1-42). At follow-up (mean 20.8 months, SD 28.7, range 0.6-64.9), two patients $(n=2$, $22.2 \%)$ recovered completely, one $(11.1 \%$ ) was functionally independent (MRS $=0-2$ ) with residual gait instability, and six $(66.7 \%)$ were functionally dependent $(\mathrm{mRS}>2)$; the most common residual deficit was cognitive impairment $(\mathrm{n}=5,55.6 \%)$.

CONCLUSION: Cerebellitis is primarily managed medically, though neurosurgical consultation is often required to evaluate for CSF diversion or surgical decompression. The

PF-037 
presence of hydrocephalus requiring neurosurgical intervention may be a predictor of severe disease and poor outcome.

Keywords: pediatric cerebellitis, pediatric neurosurgery, central nervous system infections, cerebellar disease

\section{PF-038}

\section{Innovation and technology \\ Free Flaps Cranial Vault Remodelling for Sagittal Syn- ostosis: early and late results}

Laura Grazia Valentini, Marika Furlanetto, Veronica Saletti, Mirella Seveso, Alessandra Erbetta

Fondazione IRCCS Istituto Neurologico C. Besta, Milan

OBJECTIVE: Best technique for Scaphocephaly (mininvasive vs open) correction is still matter of debeate, due to difficulties comparing aesthetic and functional results. In fact, cosmesis is hard to objectivate; cognitive and behavioural deficits are mild and need long term observation and complex testing to be quantified. Due to this uncertainty balancing the risks of intervention with the natural history, even surgical indications are discussed. We quantified results of our technique.

MATERIAL-METHODS: 148 children were operated for sagittal synostosis documented by 3DCT, with male prevalence (115/33). At surgery 123 children (A) were $<1$ year (mean 6,8 months), 25>1 year (B). Multiple craniectomies were performed with high speed drill, by zig-zag bicoronal approach; bone flaps were programmed on preoperative-CT; two broad, symmetric fronto-parieto-temporal free flaps were created, than each was divided, leaving a residual bone at vertex upon the sagittal sinus $2-4 \mathrm{~cm}$ wide, to prevent venous damage and late diastasys. To allow vertical growth, this bridge was interrupted by $2-4$ transversal cuts. Final shape was obtained by molding effect of brain growth, without rigid fixation. Continuous "Denim" suturing improved the scar.

RESULTS: There was no mortality, neither infections; 2 children were re-operated to improve cosmesis, 1 for progressive bone defect and for 1 CSF collection. Aesthetics was judged by doctors and parents in 122 children by serial photos, Eco 3D and scar quality. Neuro-psycological results were evaluated on testing and school performance, resulted in no delay (A). Improvement of shape and volume was measured by cranial index graphs and on CT/MRI. At late MRI none of 65 evaluable A children presented Chiari Malformation while it was documented in $6 / 25$ B children. CONCLUSION: The present technique had low morbidity rate and good efficacy, correcting cephalic and vertical index, preventing CM1, preserving cognition and improving aesthetics. Another advantage was feasibility in any older age.

Keywords: sagittal synostosys, scaphocephaly, cranial vault remodelling, Chiari 1 Malformation, aesthetics, cognition

\section{PF-039}

Innovation and technology

Posterior Cranial Vault Expansion_-"which technique is best?" A Comparison of the operative parameters and volume expansion following three techniques for Syndromic posterior Cranial Vault Expansion

Noor Ul Owase Jeelani ${ }^{1}$, William F Breakey ${ }^{1}$, Ezgi Mercan ${ }^{4}$, Lara S Van De Lande ${ }^{1}$, Paul G Knoops ${ }^{1}$, Justine O'hara ${ }^{5}$, Richard D Hayward $^{2}$, David J Dunaway ${ }^{3}$, Silvia Schievano ${ }^{1}$, Richard A Hopper ${ }^{4}$

${ }^{1}$ Great Ormond Street Institute of Child Health, University College of London, London, United Kingdom

${ }^{2}$ Department of Neurosurgery, Great Ormond Street Hospital, London, UK

${ }^{3}$ Department of Craniofacial Surgery, Great Ormond Street Hospital, London, UK

${ }^{4}$ The Craniofacial Centre, Seattle Childrens Hospital, Seattle, United States

${ }^{5}$ Division of Plastic Surgery, Concord Hospital, Sydney, Australia

OBJECTIVE: Posterior cranial vault expansion is now the procedure of choice to increase intra-cranial volume in patients with syndromic synostosis. It typically precedes anterior surgery due to its ability to achieve a greater volume expansion at a younger age. Our objective was to compare the operative parameters and volume expansion of three different techniques of posterior cranial expansion performed at two centers.

MATERIAL-METHODS: We retrospectively reviewed operative records and CT scans of all patients with Apert or Crouzon syndrome who underwent posterior vault expansion at our two centers. There were three treatment groups: posterior cranial vault expansion by remodeling (PCVR), distraction (PCVD) or spring expansion (PCVSE). We performed automated extraction of intracranial volume using FMRIB Software (Oxford, UK). We extracted operative parameters from the electronic medical chart and used ANOVA with post-hoc t-test analysis to compare groups. Operative parameters such as blood loss, length of stay, and operative time were a combination of the two insertion and removal operations for PCVD and PCVSE, but from the single operation for PCVR. Volume change was calculated for PCVD and 
PCVSE at the time of device removal and after the first surgery for PCVR.

RESULTS: Operative parameters and volume expansion are shown in Table 1. PCVSE had a shorter total operative time. PCVSE and PCVD had a trend for greater volume expansion than PCVR, but this did not reach significance. 1/8 of the PCVR group, 0/6 of the PCVD group, and 14/53 of the PCVSE group underwent further secondary posterior vault expansion.

CONCLUSION: The three PCV techniques reviewed had similar operative parameters and achieved comparable effective volume change. Although two surgeries were required for PSVSE and PCVD, the total surgery times were similar to the single PCVR surgery. Subsequent anterior or posterior expansion is still required in the majority of cases after initial PCV surgery.

Keywords: Cranial Vault Expansion, PVE, ICP, Springs, Distractors

\section{PF-040}

\section{Innovation and technology Surface Cranial UltraSound: the natural heir to X-Ray for the screening of skull deformities in infants}

Laura Grazia Valentini ${ }^{1}$, Marika Furlanetto ${ }^{1}$, Veronica Saletti $^{1}$, Alessandra Erbetta ${ }^{1}$, Laura Maria Pogliani ${ }^{2}$

${ }^{1}$ Fondazione IRCCS Istituto Neurologico C. Besta, Milan

${ }^{2}$ Department of Pediatrics, ASST OVESTMI, Legnano Hospital, Italy

OBJECTIVE: Currently, there is no consensus concerning the choice of a first level exam for the diagnosis of cranial deformities in infants. 3D-Computed Tomography (3D$\mathrm{CT}$ ) is considered the Gold Standard for craniosynostosis (CS), while skull X-ray is debated as a first level exam due to radiation charge, low sensitivity and specificity. Cranial ultrasound (CUS) is emerging as a non-invasive and radiation-free technique. The present study is to determine the diagnostic accuracy of CUS for CS.

MATERIAL-METHODS: The present prospective study included 350 infants (232 males, 118 females, mean age 4,4 m) referred for skull deformities. The first line exam was CUS in every case; in case of cranial suture fusion, or in case of strong clinical suspicion, they were submitted to 3D-CT. When CUS documented patent sutures and followed.We focused on infants studied both by index test and Gold Standard, performing a double-blind comparison between the echographic and the 3D-CT scan results.

RESULTS: CUS documented patent sutures in 293/350 infants; the cranial deformity improved, so confirming CUS diagnosis. In 4/350 CUS was inconclusive. In 48/350 children showed premature suture. 3D-CT confirmed the closure in 47/48, while 1 was false positive. Another 28 infants required a 3D-CT, 10 because of some doubts of closure and 18 for relevant deformity: CT confirmed CUS result (no false negatives). The sensitivity was $100 \%$, specificity $99,7 \%$, positive and negative predictive values 97,9 and $100 \%$, respectively. The CUS accuracy was $99,7 \%$.

CONCLUSION: CUS is highly sensitive and specific for CS diagnosis. It can be used in referral centres as a first level examination of skull deformities $i$, with best results obtained between 4-6 months. Its major limitations are loss of significance with increasing age and learning curve needed. Its advantages are avoidance of radiation and the chance to evaluate at the same time skull and brain.

Keywords: craniosynostosis, cranial ultrasound, suture, Computerized Tomography, infants

\section{PF-041}

\section{Moya-Moya disease \\ Can Moyamoya Suspceptible Internal Carotid Artery be Identified in Advance? - Development of a Multivariable Moyamoya Prediction Model}

\section{Shishir Kumar, Shibu V. Pillai}

Department of Neurosurgery, Narayana Institute of Neurosciences, Mazumdar Shaw Medical Centre, Narayana Health City, Bengaluru, India

OBJECTIVE: Current understanding of moyamoya vasculopathy (MMV) suggests that its development requires an initial trigger to a susceptible internal carotid artery (ICA). However, what makes an ICA susceptible and what constitutes the trigger is an open question. Recent studies have shown that morphology of vessel affects wall shear stress and can promote vessel stenosis. Studies have also shown that moyamoya ICA (mICA) differs from normal ICA (nICA) morphologically and in wall shear stress. Probably, morphology can help to characterize ICA susceptible to moyamoya. We studied infraclinoid segment of mICA, as it remains unchanged in MMV, for differences with nICA. MATERIAL-METHODS: It was a retrospective, case control study. Total 48 ICAs (24 mICA and 24 nICA) of 24 pediatric patients ( $<18$ years, 12 case and 12 controls) were studied. Anteroposterior and lateral view of MR angiogram or digital subtraction angiogram were used to study infraclinoid ICA segment. Measurements included various angles, their ratios and tortuosity. Measurements having significant differences $(\mathrm{P}<0.05)$, were further analyzed for their ability to discriminate between two groups. Those with c statistics $>0.7$ were chosen as predictors. Using these predictors, 
a point-based model was developed moyamoya predictive model (MPM).

RESULTS: There were significant differences in infraclinoid segment of mICA and nICA, with mICA vessels being less tortuous and straighter in their petrous and gasserian clival segment course $(\mathrm{P}<0.01)$. MPM developed from the identified predictors was able to discriminate between mICA and nICA (c statistic $=0.994$ ).

CONCLUSION: MPM convincingly differentiates mICA and nICA. It can be used to characterize ICA having mICA morphology whenever an angiogram is done for patients. These patients can be followed, after some trigger viz infection, autoimmune disorder etc. for MMV development. Further, studies are required to validate the model and determine the duration of follow up for various triggers.

Keywords: moyamoya, ICA susceptibility, Predictive model

\section{PF-042}

\section{Moya-Moya disease \\ Current trends in Pediatric Moyamoya: A survey of International Practitioners}

Harishchandra Lalgudi Srinivasan ${ }^{1}$, Moran Hausman Kedem $^{2}$, Edward R Smith ${ }^{3}$, Shlomi Constantini ${ }^{1}$, Jonathan Roth $^{1}$

${ }^{1}$ Pediatric Neurosurgery Department, Dana-Dwek Children's Hospital, Tel Aviv Sourasky Medical Center, Tel Aviv, Israel ${ }^{2}$ Pediatric Neurology Institute, Dana-Dwek Children's Hospital, Tel Aviv Sourasky Medical Center, Tel Aviv, Israel

${ }^{3}$ Pediatric Cerebrovascular Surgery, Pediatric Neurosurgery, Children's Hospital Boston / Harvard Medical School, Boston, MA, USA

BACKGROUND AND AIM:Moyamoya angiopathy (MM) is a chronic, progressive steno-occlusive arteriopathy of the distal internal carotid artery and its proximal branches. MM is recognized as a shared end-pathway common to a broad range of inciting pathologies, suggesting that tailored management is important. Pediatric MM differs from MM in adults. Currently, there are many uncertainties and controversies regarding the diagnosis and management of children with MM. Hence, we conducted an international survey to identify the contemporary management trends followed worldwide.

METHODS:A survey relating to lifestyle modifications, medical management, diagnosis, surgical management, and follow up for pediatric MM was circulated across web-based platforms, through various international pediatric neurological and neurosurgical societies. Data collected included geographic region of practice, experience, responses to questions, and comments.
RESULTS: 127 responses were evaluated (104 neurosurgeons and 23 neurologists, from 32 countries, across 6 continents). We found wide variations in the recommendations for management and life-style modification, with significant differences between regions of practice. $80 \%$ recommend restrictions on physical activity, particularly for symptomatic and non-operated patients. $84 \%$ prescribe aspirin, and $80 \%$ continue aspirin following surgery. $65 \%$ perform indirect revascularisation. $78 \%$ recommend performing a staged surgery for bilateral MM. Only $26 \%$ perform acetazolamidechallenge SPECT to evaluate brain perfusion.

CONCLUSIONS:This survey reflects the contemporary trends in management of pediatric MM, while highlighting the heterogeneity in the management approach of these patients. There is a need for multi-center, international studies to evaluate the safety, efficacy, and long-term outcome of various aspects of treatment of these patients.

Keywords: Pediatric Moyamoya; Moyamoya survey; pediatric stroke; moyamoya treatment; moyamoya lifestyle modifications

\section{PF-043}

\section{Neuro-Oncology \\ Proteomic analysis of pediatric ependymomas}

Luca Massimi ${ }^{1}$, Diara Valeria Rossetti ${ }^{2}$, Claudia Martelli ${ }^{2}$, Federica Vincenzoni ${ }^{2}$, Susanna Di Silvestre ${ }^{2}$, Gianluca Scorpio $^{2}$, Federico Bianchi ${ }^{1}$, Gianpiero Tamburrini ${ }^{1}$, Andrea Urbani $^{3}$, Claudia Desiderio ${ }^{4}$

${ }^{1}$ Neurochirurgia Pediatrica, Fondazione Policlinico Universitario A. Gemelli IRCCS, Roma, Italy

${ }^{2}$ Istituto di Biochimica e Biochimica Clinica, Università Cattolica del Sacro Cuore, Roma, Italy

${ }^{3}$ Area Diagnostica di Laboratorio, Fondazione Policlinico Universitario Agostino Gemelli-IRCCS, Roma, Italy

${ }^{4}$ Istituto di Chimica del Riconoscimento Molecolare, Consiglio Nazionale delle Ricerche, Roma, Italy

OBJECTIVE: The management of ependymomas still rely mainly on surgery, being these tumors resistant to radiotherapy and, in particular, to common chemotherapic agents. Surgery may be particularly complicated by the tendency of the so called called Group A (posterior fossa ependymomas) to affect small children and extend to the cerebello-pontine angle embracing cranial nerves. A proteomic characterization could therefore help in finding possible prognostic differential factors among tumors of different grades and localization.

MATERIAL-METHODS: The intact and digested proteome of 12 pediatric ependymomas of different grade and localization have been analyzed by the top-down and the shotgun 
proteomic approaches after tissue homogenization of the acid-soluble and the acid-insoluble fractions resulting from centrifugation (HPLC-Orbitrap Elite MS).

RESULTS: Together with profiling a common signature of ependymoma tumor tissues, the obtained data evidenced interesting differentiations between Who II and III tumor grades following both proteomic approaches. Particularly, top-down proteomics distinguished the grade III tissue specimens for the down-expression of proteins involved in oxidative metabolism while shotgun data evidenced the exclusive classification of pathways involved. Gene ontology classification of the proteins identified with confidence by the shotgun strategy evidenced the pathways connected to de novo pyrimidine deoxyribonucleotide biosynthesis and ribonucleotides salvage, response to oxidative stress and hypoxia, blood coagulation and cell cycle as distinguishing WHO grade III tissues.

CONCLUSION: This is a first, preliminary step toward the knowledge of the proteomic behavior of pediatric ependymomas. Further considerations based on the diverse tumor localization (group A and B subtentorial ependymomas) and supratentorial ependymoma are under preliminary evaluation.

Keywords: ependymomas, proteomics

\section{PF-044}

\section{Neurotrauma/Critical Care \\ Management and outcome of paediatric head injury caused by falls in sub-Saharan Africa}

Muhammad Koko Aliyu, Nasiru Jinjiri Ismail, Ali Lasseini, Bello Bala Shehu

Department of Neurosurgery, Regional center for Neurosurgery, Usmanu Danfodiyo University Teaching Hospital Sokoto, Sokoto State, Nigeria

OBJECTIVE: The profile and outcome of fall-associated head injury in sub-Saharan Africa may vary from other part of the world, due to environmental and socio-cultural peculiarities. Despite the burden of head trauma resulting from falls in the region, there are few studies in the literature about this topic. The objectives of the study were to highlight the pattern and management outcome of falls-related head injury in children in sub-Saharan Africa.

MATERIAL-METHODS: This is a retrospective hospitalbased study of children ( $\leq 18$ years) admitted to neurosurgery department with head injury resulting from falls between August 2015 to July 2017. Bio-demographics, clinical details were analysed using SPSS. IBM version 20.0 for windows.
RESULTS: Out of the total sixty-two patients, fifty-six (90.3\%) were males. The mean age of presentation was eight \pm 2 years. Falls from tree was the most common cause (69.4\%); other falls were from buildings and stationary trucks. The main reasons for climbing the trees were to pluck fruits and leaves for human and animal consumption. Posttraumatic seizure was found in $24.2 \%$ and $33.9 \%$ presented with additional other injuries (30.6\% for limb fractures and $3.3 \%$ had spinal cord injury). Fifty-three percent presented with Glasgow coma score between $9-13$ and $40.3 \%$ of the patients underwent operative treatment. The mean duration of hospital stay was $21 \pm 9$ days. There was good recovery (Glasgow outcome score of four and above) in $66.7 \%$ and mortality was found in $21.4 \%$. Although, the mortality was higher in males, age $\leq 5$ years and patients that had nonoperative treatment, there was no statistical significance. CONCLUSION: Disability and death caused by falls is a major health problem in sub-Saharan Africa. Public health awareness and strict laws protecting children's safety are advocated to reduce the occurrence of this challenging problem in the society.

Keywords: paediatric head injury, outcome, management, falls

\section{PF-045}

\section{Neurotrauma/Critical Care \\ Paediatric Neurosurgical Trauma due to fall from Win- dows: a 5 year retrospective review in a tertiary care Paediatric Trauma Center}

\author{
Vikrant Setia $^{1}$, Ajay Sinha ${ }^{2}$, Siddarth Vankipuram ${ }^{3}$ \\ ${ }^{1}$ Vikrant Setia, Department of Neurosurgery, Alder Hey \\ Children Hospital, Liverpool, United Kingdom \\ ${ }^{2}$ Ajay Sinha, Department of Neurosurgery, Alder Hey Chil- \\ dren Hospital, Liverpool, United Kingdom \\ ${ }^{3}$ Siddarth Vankipuram, Department of Neurosurgery, Alder \\ Hey Children Hospital, Liverpool, United Kingdom
}

BACKGROUND AND AIM:Trauma is one of the major cause of death in children $>1$ year of age in the UK, and falls are a common cause of this trauma.

Falls from windows contribute a significant percentage of this trauma and are often overlooked. Window falls are important as they can lead to severe injuries however are completely preventable and are subject of public health importance. The aim of this study was to identify the:

Prevalence of Paediatric Window Falls

Types of Neurosurgical injuries among children who fell from windows

Circumstances leading to window fall 
Associated injuries other than Neurosurgical trauma METHODS:It was a retrospective analysis. All paediatric patients presenting to Alder Hey Childrens NHS trust A and E with a history of injuries related to unintentional window falls between April 2015 and June 2020 were included in the study.

RESULTS: Window falls contributed $19 \%$ of the total Neurosurgical emergency admissions related to fall with the most common age of presentation being between 4-5 and 5-6 yrs age respectively. The majority of the patients presented with an Injury Severity Score $>15$ and the most common Neurosurgical injury susatined was Fracture with decompressive craniectomy being the commonest Neurosurgical Procedure. The most common floor of fall was 1st floor. Polytrauma, involving more than 3 systems was reported in approximately $12 \%$ of these patients. There was mortality rate of $12 \%$ among these patients. Economical burden was significant with almost $50 \%$ patients having permanent disability requiring support. Psychological trauma was the major long term issue in these patients.

CONCLUSIONS:In children falls from relatively modest heights are associated with significant morbidity, and interventions to prevent them are warranted. Hence Window falls prevention programmes, and local efforts based on enforcement of installation of window locks, can be used to decrease incidence of pediatric window falls in urban centers.

Keywords: Window, Falls, Neurosurgical, Injuries

\section{PF-046}

\section{Neurotrauma/Critical Care \\ Disturbances of functional connectivity between default mode network and cerebellar structures in patients with mTBI in acute stage. rsfMRI and DTI study}

Maxim Ublinskiy ${ }^{1}$, Andrei Manzhurtsev ${ }^{1}$, Tolibdzhon Akhadov $^{1}$, Natalia Semenova ${ }^{2}$, Alexei Yakovlev ${ }^{3}$.

${ }^{1}$ Department of Radiology, Clinical and Research Institute of Emergency Pediatric Surgery and Trauma (CRIEPST), Moscow, Russian Federation

${ }^{2}$ Institute of Biochemical Physics, Russian Academy of Sciences, Moscow, Russian Federation

${ }^{3}$ Lomonosov Moscow State University, Moscow, Russian Federation

BACKGROUND AND AIM: Mild traumatic brain injury (mTBI) occupies one of the first places in children injuries. Among all brain networks at the resting state, the Default Mode Network (DMN) is the most widely studied network. The aim of this study is to examine functional connectivity in normal-appearing cortex in acute period of mTBI using rsfmRI and DTI.
MATERIAL-METHODS: 34 MR negative participants were studied in age from 12 to 17 years (mean age -14.5 years). Group of patients consisted of 17 children with mild traumatic brain injury in acute stage. 17 age-matched healthy volunteers comprised control group. All studies were performed at Phillips Achieva 3.0 T MRI scanner using 32-channel head coil. fMRI data were processed using functional connectivity toolbox CONN. Seed-based analysis was performed in order to reveal disturbances in functional connectivity.Statistical processing was performed using Statistica 12 .

RESULTS: DTI analysis didn't show any changes in values of apparent diffusion coefficient (ADC) and fractional anisotropy (FA) between two groups (see Fig. 1). No statistically significant differences in correlation strength between DMN parts were observed in two groups (see Fig. 2). Intergroup seed-based analysis revealed statistically significant $(\mathrm{p}<0,05)$ difference in neural correlations between DMN parts and vermis (cerebellum structural part): positive link in control group and negative link in group of patients. CONCLUSIONS: One of the most common symptoms of mTBI is dizziness as a result of impaired movements coordination. Vermis as an essential cerebellum part plays an important role in the vestibulo-ocular system which is involved in the learning of basic motor skills in the brain. Vermis aids in the synchronization of eye and motor functions in order for the visual field and the motor skills to function together. Our results show that mTBI appears to be a possible reason of connectivity malfunction in normalappearing vermis.

Keywords: DTI, MRI, rsFMRI, mTBI, cerebellum, vermis

\section{PF-047}

\section{Occult tethered cord}

Re-tethered cord syndrome in paediatric patients with conus lipoma: the second surgery

Rina Agushi ${ }^{1}$, Timothée De Saint Denis ${ }^{1}$, Michel Zerah ${ }^{1}$, Syril James ${ }^{1}$, Pauline Lallemant ${ }^{2}$, Veronique Forin ${ }^{2}$

${ }^{1}$ Neurochirurgie Pédiatrique, Hôpital Necker, APHP, Centre de Référence C-MAVEM, Université de Paris, 149 rue de Sèvres, 75,015, Paris, France

${ }^{2}$ Department of Pediatric Physical Therapy and Rehabilitation, APHP, Centre de Référence C-MAVEM, Sorbonne Université, Paris, France

OBJECTIVE: Spinal lipomas are among the most common form of closed spinal dysraphism. They encompass a range of clinical significance from asymptomatic to profound neurological impairment. An important sub-entity is represented by the conus lipomas, which as shown by several studies, 
represent still a controversial argument in terms of surgical indication, timing of surgery, technique and long-term follow-up. The aim of our study was to analyze the clinical and radiological reasons for re-tethering, and thus the outcome of an eventual second surgery.

MATERIAL-METHODS: For this retrospective study, the databank of the "Hôpital Necker Enfants Malades" was used. 165 consecutive operated on patients, with age between 0-16 years, diagnosed with a conus lipoma, in a period going from January 2000-January 2020, were included. The NEM scale was used for the neurological and clinical evaluation of the patients, as well as routine bladder ultrasound and urodynamic studies.

RESULTS: The mean follow-up was of 9.5 years ( \pm 0.7 years). $7.2 \%$ of the patients needed re-surgery. All of these patients presented with a chaotic voluminous lipoma, corresponding to type 2 and 3 of spinal lipomas (Morota classification). The presence of pre-op syringomyelia did not result statistically significative in influencing the predisposition for a second surgery. The clinical reasons bringing to re-surgery consisted in urologic degradation $(75 \%$ of the patients), followed by bowel dysfunction (40\%), and lastly pain was only present in $25 \%$ of the cases. The outcome of the second surgery can be reassumed as follows, $41 \%$ of the patients improved in terms of sphincter control, 50.7\% remained stable, $8.3 \%$ worsened. None of the patients underwent a third intervention.

CONCLUSION: According to our experience the re-tethering depends mostly upon the specific radiological pre-op features.

Keywords: spinal lipoma, occult tethered cord, re-tethered cord syndrome, closed spinal dysraphism, neurologic bladder

\section{PF-048}

\section{Other}

Pediatric Cranial Deformations: Demographic Associations

Jarrett Foster ${ }^{1}$, Ranbir Ahluwalia ${ }^{1}$, Madeleine Sherburn ${ }^{1}$, Katherine Kelly ${ }^{1}$, Georgina Sellyn ${ }^{1}$, Chelsea Kiely ${ }^{1}$, Alyssa Wiseman ${ }^{1}$, Stephen Gannon ${ }^{1}$, Chevis Shannon ${ }^{1}$, Christopher Bonfield $^{2}$

${ }^{1}$ Surgical Outcomes Center for Kids, Monroe Carell Jr. Children's Hospital at Vanderbilt, Nashville, TN, USA

${ }^{2}$ Department of Neurological Surgery, Vanderbilt University Medical Center, Nashville, TN, USA

OBJECTIVE: The connection of Back to Sleep Movement and cranial deformation has been outlined, considerations toward cultural or anthropologic difference should also be investigated. This paper investigates differences in cranial measurements between gender, race, and ethnicity in our population

MATERIAL-METHODS: Retrospective review of 1,499 pediatric patients, presenting with a negative CT scan in 2018 was conducted. Cranial Vault Asymmetry Index (CVAI) and Cranial Index (CI) evaluated potential cranial deformations. The cohort was evaluated for differences between gender, race, and ethnicity among 1) all patients and 2) patients within the clinical treatment window (aged 2-24 months).

RESULTS: In the overall CVAI cohort, $56.7 \%$ were male, 72\% Caucasian, $17.7 \%$ African American (AA), and 10.3\% Hispanic/Latin American (H/LA) descent (10.3\%). Mean CVAI were significantly different between genders and race. However, only race was associated with differences in positional posterior plagiocephaly (PPP) diagnosis. The H/ LA subgroup showed no differences in CVAI measurement. Of the 520 patients in the treatment window cohort, 59\% were male, $64.2 \%$ Caucasians, $15.4 \% \mathrm{AA}$, and $10.7 \% \mathrm{H} / \mathrm{LA}$ patients. There were no differences between mean CVAI values for gender or $\mathrm{H} / \mathrm{LA}$ descent. There were significant differences between Caucasian and AA mean CVAI values and rate of PPP diagnosis. In the total CI cohort, 56.6\% were male, $67.1 \%$ Caucasians, $16.5 \% \mathrm{AA}$, and 9.2\% H/LA descent. Within the clinical treatment window cohort, 59.2\% were male, $80.1 \%$ Caucasian, $19.9 \%$ AA, and $10.4 \%$ H/LA. Mean CI values were not significantly different between genders in either cohort. However, there were significant differences between CI measurements for Caucasians and AA patients as well as patients of H/LA descent in both cohorts. CONCLUSION: There are no significant associations between cranial deformations and gender. However, significant differences exist between Caucasian/African American patients as well as patients with Hispanic/Latin American heritage. These findings suggest cultural or anthropological influences on defining skull deformations.

Keywords: plagiocephaly, brachycephaly, cranial deformity, demographics

PF-049

\section{Other}

Quality of Life and Satisfaction in Surgical versus Conservative Treatment of Non-Syndromic Children with Craniosynostosis

Nicholas Sader ${ }^{1}$, Vivek Mehta ${ }^{2}$, Shannon Hart $^{3}$, Lori Bliss ${ }^{2}$, Hanna Moore $^{2}$, Melissa Dasilva ${ }^{2}$, Ruksana Rashid ${ }^{1}$, Jay Riva Cambrin $^{1}$

${ }^{1}$ Department of Neurosurgery, University of Calgary, Calgary, Canada 
${ }^{2}$ Department of Neurosurgery, University of Alberta, Edmonton, Canada

${ }^{3}$ Faculty of Medicine, Memorial University, St. John's, Canada

OBJECTIVE: Craniosynostosis represents the second most common reason for referral to pediatric neurosurgery. However, the quality of life and neurodevelopmental impact of leaving this physical disorder uncorrected is poorly understood.

MATERIAL-METHODS: This multicenter cross-sectional study identified previously managed non-syndromic infants ( $<24$ months of age) with single-suture craniosynostosis at both pediatric neurosurgical centers in Alberta, Canada. The primary variable of interest was the allocated treatment (surgical versus conservative). The primary outcome was the PedsQL, a validated measure of quality of life looking at physical, emotional, social, and school functioning. Caregivers were asked the reasons for selected management and treatment decision satisfaction.

RESULTS: 114 children met the inclusion criteria. $78 \%$ had surgery, with twenty-five (22\%) having conservative treatment. The most common suture affected was sagittal (54\%), followed by metopic (33\%), coronal (10\%), and lambdoid (3\%). Caregivers most commonly opted for surgery because of severe appearance (80\%). $76 \%$ and $72 \%$ of the caregivers of children with conservative management did so due to concerns for surgical risks and mild appearance, respectively. There was a statistically significant relationship between both the parent's $(\mathrm{p}<0.001)$ and the surgeon's $(p<0.001)$ impression of head shape severity and treatment decision. Parent's satisfaction with their child's appearance as well as satisfaction for their treatment decision did not differ between treatment groups. Regarding quality of life, on univariate analysis, the conservative group had a statistically higher physical score $(\mathrm{p}=0.01)$, psychosocial score $(\mathrm{p}=0.004)$, and total mean scale score $(\mathrm{p}=0.003)$ compared to the surgical group. However, when adjusting for severity and age at consult, there is no significant association between treatment and all PedsQL scale scores.

CONCLUSION: Alberta families have a higher number of children with craniosynostosis treated with conservative management compared to most American studies. We found no independent association between quality of life and treatment allocation.

Keywords: craniosynostosis, pediatric, quality of life, pedsQL, satisfaction

\section{PF-050}

\section{Other}

Results of the surgical treatment in children with Chiari 1 malformation: Oulu experience

Niina Salokorpi ${ }^{1}$, Minna Vakkuri ${ }^{2}$, Maija Lahtinen ${ }^{1}$, Juho Nissilä5, Miro Pekka Jussila ${ }^{2}$, Willy Serlo ${ }^{4}$, Maria Suo Palosaari $^{3}$

${ }^{1}$ Department of Neurosurgery, Oulu University Hospital, Oulu, Finland

${ }^{2}$ Oulu University, Oulu, Finland

${ }^{3}$ Department of Pediatric Radiology, Oulu University Hospital, Oulu, Finland

${ }^{4}$ Department of Pediatric Surgery, Oulu University Hospital, Oulu, Finland

${ }^{5}$ Department of Radiology, Oulu University Hospital, Oulu, Finland

OBJECTIVE: There is still no consensus on what surgical technique is optimal in the surgical treatment for the Chiari malformation 1 (CM1). We present our institutional experience with CM1 management by bony decompression and splitting of the superficial layer of the dura without duraplasty.

MATERIAL-METHODS: We evaluated all the pediatric cases operated due to CM1 in the Oulu University Hospital since 2007 until 2019. During these years a 31 consecutive patient was operated. The primary operation was in all cases bony only decompression (enlargement of the foramen magnum with removal of posterior arches of $\mathrm{C} 1$ and $\mathrm{C} 2$, if necessary) with longitudinal splitting of outer dural layer without duraplasty.

RESULTS: Mean age at operation was 8.4 years. The main indication for surgery was scoliosis in 3 patients, with clinical symptoms (incl. headaches, balance disturbances, muscular weakness, numbness of limbs), supported in some cases by pathological findings on electrophysiological examinations, being an indication in the rest of patients. Also, in 13 patients syringomyelia was present. No patients were operated missing these signs and/or symptoms. There were no complications requiring surgical intervention. Four patients (13\%) needed further operations. In one patient syringomyelia was drained years after the primary surgery. In the other three cases a re-do surgery was required due to progression of the symptoms. Thus stabilization or improvement of the symptoms was seen in $87 \%$ of the cases after the primary operation. After re-do surgery improvement was seen in all three patients. As a result, after average of 4.5 years of follow-up, there was significant improvement $91 \%$ of the patients.

CONCLUSION: Bony only decompression with longitudinal split ting of outer dural layer without duraplasty is safe and efficient technique of choice for primary surgery in CM1. Though in some cases re-do surgery with more aggressive approach could be required on follow-up. 
Keywords: Chiari Malformation Type I, bony-only, outcome, syringomyelia, management

\section{PF-051}

\section{Other}

Quantitative Parcellation of the Dentato-RubroThalamo-Cortical Tract according to Frontal Connectivity

\section{$\underline{\text { Sebastian M Toescu }}^{1}$, Patrick W Hales ${ }^{2}$, Christopher A Clark $^{2}$, Kristian Aquilina ${ }^{1}$ \\ ${ }^{1}$ Department of Neurosurgery, Great Ormond Street Hospi- tal, London, UK, WC1N 3JH \\ ${ }^{2}$ Developmental Imaging and Biophysics Section, UCL- GOS Institute of Child Health, 30 Guilford St, London, UK, $\mathrm{WC} 1 \mathrm{~N} 1 \mathrm{EH}$}

OBJECTIVE: The dentato-rubro-thalamo-cortical (DRTC) tract is the main outflow pathway of the cerebellum. The precise termination of the DRTC in the frontal lobes of developing children has been unclear. We sought to probe the frontal connections of this tract using diffusion MRI (dMRI), hypothesising a significant connection to cortical regions consistent with the supplementary motor area.

MATERIAL-METHODS: 30 healthy children underwent volumetric T1-weighted and multi-shell $(b=0,1000$, $2200 \mathrm{~s} / \mathrm{mm} 2$ ) dMRI scanning. After dMRI preprocessing with standard FSL / MRtrix tools, manual tractography was performed to segment the DRTC tract using fibre orientation distributions derived from constrained spherical deconvolution modelling. Cortical regions were extracted from T1 images linearly registered to dMRI, using Freesurfer parcellation. The intersection of cortex and streamlines were plotted on a group template map in dMRI space. The number of tractography streamlines terminating in subregions of the frontal and central cortex were normalised by total streamline count per tract and by volume of subregion. One-way analysis of variance and multiple comparison tests were used to identify differences in streamline counts.

RESULTS: Subjects had a mean age of 11.7 (S.D. 3.82); 16 were female. Figure 1 shows the results of unconstrained DRTC streamline termination. The highest density appears to be in M1, followed by the SMA extending rostrally along the superior frontal gyrus. Quantitative analysis of normalised streamline terminations is shown in Fig. 2. There was a significant difference between streamline counts in frontal subregions for left $(\mathrm{p}=7 \times 10-12)$ and right $(\mathrm{p}=0.0005)$ DRTC tracts. The pre-central gyrus had a significantly higher normalised streamline count than other regions for both DRTC, followed by the superior frontal gyrus and pars orbitalis.
CONCLUSION: The predominant cortical projections of the DRTC are to motor and speech areas. These results may be of relevance in the study of children following acquired cerebellar damage such as cerebellar mutism syndrome.

Keywords: Diffusion tractography, cerebellar mutism syndrome, cerebellar projections, quantitative MRI

\section{PF-052}

\section{Other}

\section{How To Undertake a Craniopagus Twin Separation- "Think twice before you cut"}

$\underline{\text { Ido Ben Zvi }}{ }^{1}$, David Dunaway ${ }^{2}$, Juling Ong ${ }^{2}$, Curtis Budden $^{2}$, Owase Jeelani ${ }^{1}$

${ }^{1}$ Paediatric Neurosurgery Department, Great Ormond Street Hospital for Children, London, United Kingdom

${ }^{2}$ Paediatric Plastic Surgery Department, Great Ormond Street Hospital for Children, London, United Kingdom OBJECTIVE: Craniopagus twins (CPT) are the rarest form of conjoined twins, occurring at a rate of 0.6 per 1 million births, with a female-male ratio of $4: 1$. Reports in the literature are limited to case reports. The level of involvement of anatomical structures in the union can vary from skin and skull alone, to joined venous sinuses and cerebral parenchyma. The separation of CPT is extremely difficult with high mortality and morbidity rates. Great Ormond Street Hospital (GOSH) has the largest series reported, with four CPT sets successfully separated, between 2006 and January 2020.

The strategy at GOSH for CPT separation is a combination of multi-disciplinary team effort, and a carefully planned staged operation. The team consists of neurosurgeons, plastic surgeons, anesthetists, intensive-care physicians, specialized nursing staff, bio-engineers and management and marketing personnel.

MATERIAL-METHODS: Planning of such an operation necessitates several months. Major details such as how the skin, bones and venous structures would divide, as all pairs of CPT in our series shared venous sinuses, and minor details such as the type of surgical table, the equipment to be used for stealth navigation and planning of a postoperative space that allows the twins to sleep together for stress alleviation. Evolution between operations prompted the introduction of 3-D printer models for the planning of the second CPT separation, and the insertion of tissue expanders as the first step, instead of the third (last separation), which allows to shorten significantly the total time of completion of the separation. 
RESULTS: All patients survived. Two patients underwent venous infarcts and four patients need chronic physical and/ or cognitive assistance.

CONCLUSION: Management of venous drainage is the single most critical outcome factor. A staged operation allows for collateral veins to develop, contributing to patient outcome. A dedicated, experienced multidisciplinary team is vital for the success of these operations.

Keywords: Craniopagus, conjoined, staged operation

\section{PF-053}

\section{Other}

Quantification of Skull Shape and Size for springassisted correction in Patients with Isolated Lambdoid Craniosynostosis

Lara S. Van De Lande ${ }^{1}$, Selim Bozkurt ${ }^{3}$, Hamad Khan ${ }^{1}$, Eimear O' Sullivan ${ }^{2}$, Alessandro Borghi ${ }^{1}$, Juling Ong ${ }^{1}$, Silvia Schievano $^{1}$, Gregory James ${ }^{1}$, David J. Dunaway ${ }^{1}$, N. U. Owase Jeelani ${ }^{1}$.

${ }^{1}$ Craniofacial Unit, Great Ormond Street Hospital, London, UK \& Institute of Child Health, University College London, UK

${ }^{2}$ Department of Computer Vision, Imperial College, London, UK

${ }^{3}$ Institute of Cardiovascular Sciences, University College London, London, UK

OBJECTIVE: Isolated lambdoid craniosynostosis (ILC) is a rare type of premature fusion of the cranial sutures resulting in ipsilateral occipital flattening and compensatory contralateral bossing of the parietal bone. Different surgical techniques, including partial or complete cranial vault remodeling, endoscopic strip suturectomy, distraction osteogenesis, and spring-assisted cranioplasty (SAC) have been utilised to correct their head shape. SAC has the advantages of being minimally invasive with less blood loss and transfusion requirements, and reduced hospital stay compared to total vault remodeling. However, aesthetic outcomes of SAC may remain suboptimal due to rapid growth of the skull at early ages, changes in the bone and suture properties, and the limited deformation vectors provided by the springs. Understanding the 3D shape change in the skull of ILC patients after SAC may enhance the surgical outcomes. The aim of this study is to quantify change between the pre- and postoperative skull shapes, and intracranial volumes.

MATERIAL-METHODS: Five patients with average age of $7.4 \pm 3.1$ months were included. Pre- and postoperative computed tomography scans were reconstructed to 3D skull models. Patients' baseline characteristics were collected. Semi-automated quantification using non-rigid iterative closest point registration (NICP) was used to analyse the shape differences of the pre- to postoperative skull models. Finite element modelling (FEM) was performed to quantify change in the cranial volumes.

RESULTS: Computerised models show that maximal displacement in the osteotomised bones and intracranial volume expansion depend on the size of cuts. One example case is shown in Fig. 1, demonstrating the calvarial bone changes and the intracranial volume analysis using NCIP and FEM, respectively. Overall, bone displacement may reach up to $40 \mathrm{~mm}$ together with $300 \mathrm{~mL}$ volume increase.

CONCLUSION: The postoperative shape changes as a result of SAC in ILC were successfully quantified and the results will be used for optimisation of the future surgical outcome.

Keywords: Craniosynostosis, corrective surgery, quantification, head shape, craniofacial surgery, spring assisted cranioplasty

\section{PF-056}

\section{Other}

Part 1: Surgical Correction in 231 Trigonocephaly Patients - The Alder Hey Experience

Anusha A Hennedige, Christopher Parks, Jonathan Ellenbogen, Ajay Sinha, Christian Duncan, David Richardson Alder Hey Children's Hospital, Department of Craniofacial Surgery, Liverpool, England

BACKGROUND AND AIM: Isolated metopic synostosis presents with a range of severity, from a palpable ridge as the sole presenting feature to a constellation of features resulting in trigonocephaly. At our unit, patients on the moderate to severe end of the phenotypic spectrum of trigonocephaly are offered fronto-orbital advancement and remodelling (FOAR).

METHODS: A retrospective data collection was performed. All trigonocephaly patients who were operated on at Alder Hey Children's Hospital in Liverpool between January 2000 to January 2020, were identified via the hospital's coding department and cross-referenced with individual surgeon's record of their own operations.

RESULTS: Over 20 years, we operated on 231 patients with trigonocephaly. The average age at surgery was 18 months, with an average follow-up of 77.4 months. Ten percent of patients sustained a dural tear with no long-term consequences. The total early complication rate was $12.1 \%$. The most common early complications were wound infection and wound dehiscence. The total reoperation rate was $6.5 \%$. The introduction of infection prevention and control measures over the two decades at our unit reduced the reoperation rate to $1.1 \%$. The recurrence rate of a metopic ridge was 
$2.3 \%$ with no patients requiring further surgery. None of our patients required calvarial remodelling for raised ICP after FOAR.

CONCLUSIONS: This study presents the largest outcome series of isolated trigonocephaly patients. Despite operating on patients at an older age compared to other series, we have demonstrated similar or lower complication and reoperation rates with no patients requiring further calvarial remodelling for raised ICP. Infection is the commonest early complication and through evolution in our practice, we have reduced our complication rate dramatically. We present recommendations which include an infection control care bundle, cessation of surgical drains and practice adjustments to reduce risks of infection and risk of requiring further calvarial remodelling for raised ICP.

Keywords: Trigonocephaly, Metopic, Craniosynostosis

\section{PF-057}

\section{Spine}

Atlantoaxial Rotatory Fixation in Childhood: A Management Guideline Based on 17 Years of Quaternary Centre Experience

Ciaran Scott Hill, Anouk Borg, Dominic Thompson

Great Ormond Street Hospital, London, UK

OBJECTIVE: Atlantoaxial Rotatory Fixation (AARF) is an acquired childhood condition that typically presents with painful torticollis. Incorrect and delayed diagnosis are commonplace and management strategies are poorly defined.

The purpose of this study is to identify predictors of nonsurgical treatment success and failure, so as to refine a pragmatic stepwise treatment strategy.

MATERIAL-METHODS: Retrospective analysis of all cases of AARF treated in a quaternary paediatric neurosurgery centre between January 2003 and January 2020. Treatment failure was defined as the requirement to proceed to open surgery, or the re-occurrence of AARF within 3 months of previously proven correction.

RESULTS: Data was available for 33 patients treated for AARF. Patients were divided into Groups 1 or 2 depending on whether they were initially manged with a collar or a halo brace. Patients who relapsed after management with a collar were then treated with a halo brace. The combined success rate of non-surgical strategies was $70 \%$. Ten patients required surgical fixation (30\%), six were from Group 1 and four from Group 2 and none of these recurred. Late presentation was associated with treatment failure.

CONCLUSION: Early diagnosis and effective initial reduction are key to successful management of AARF in childhood. Children who present late have a higher relapse rate after immobilisation by either collar or halo and are more likely to require internal fixation. In our series the majority of children (70\%) can be successfully treated without recourse to open surgery.

Keywords: Atlanto-axial, Rotatory, Torticollis, cock-robin, collar, halo

\section{PF-058}

\section{Spine}

Management of Chiari malformation in syndromic and non-syndromic craniosynostosis

\section{Hideki Ogiwara}

Department of Neurosurgery, National Center for Childs Health and Development, Tokyo, Japan

OBJECTIVE: Although association of Chiari malformation (CM) and craniosynostosis is well-recognized, its management remains controversial. There are differences in the clinical course of CM in syndromic craniosynostosis (SC) and non-syndromic craniosynostosis (NSC). Whether cranial expansion surgery, foramen magnum decompression (FMD), or both should be conducted and when would be the appropriate timing are still unclear. Here, we retrospectively reviewed the clinical data at our institution to investigate the optimal management of CM associated with SC and NSC. MATERIAL-METHODS: Clinical data of 163 children with craniosynostosis who underwent surgical treatment at National Center for Child Health and Development between April 2002 and May 2018 were retrospectively analyzed. RESULTS: Twelve out of 119 children (10\%) with NSC and 14 out of 44 children (31.8\%) with SC were radiologically diagnosed with CM. Median age at which CM was radiologically diagnosed was 7 months. Out of the 12 NSC cases with CM, 1 (8\%) was symptomatic with gait disturbance. This patient underwent FMD following expansion surgery. Out of the $14 \mathrm{SC}$ cases with CM, 8 (57.1\%) were symptomatic with central sleep apnea. For SC cases with symptomatic $\mathrm{CM}$, cranial expansion surgery alone was conducted in 2 cases, FMD was conducted after cranial expansion in 4 cases, and FMD was conducted first and additional expansion surgery was conducted in 2 cases. In all cases, symptoms of CM improved after completing these series of surgeries. Regarding asymptomatic CM, cranial expansion surgery alone was performed and none of them showed symptoms of CM thereafter in both SC and NSC.

CONCLUSION: Multiple surgeries tend to be necessary for symptomatic CM in both syndromic and non-syndromic craniosynostosis, while all asymptomatic $\mathrm{CM}$ did not become symptomatic after cranial expansion surgery. 
Keywords: syndromic craniosynostosis, Chiari malformation, cranial expansion, foramen magnum decompression

\section{PF-059}

\section{Spine}

Instrumentation of the Paediatric Craniovertebral Junction: A Review of Practice

Isabel Tulloch, Fozia Saeed, Christopher Derham, Gnanamurthy Sivakumar, Paul Chumas, John Goodden, Atul Tyagi Department of Neurosurgery, Leeds Childrens Hospital, Leeds, United Kingdom

OBJECTIVE: Surgical management of paediatric craniovertebral junction instability poses a unique set of challenges. Sparse paediatric-specific data on techniques and outcomes of spinal instrumentation has meant that outcome inferences are typically made from adult series'. However, children's petite and frequently fragile osseous and ligamentous structures, alongside variations in anatomy associated with syndromic craniovertebral abnormalities, limit these inferences and can render these procedures challenging. We review our experience of craniovertebral instrumentation over the past twelve years.

MATERIAL-METHODS: Retrospective review March 2008-2020 identifying 21 patients. Data collected on demographics, type of intervention performed and treatment outcomes.

RESULTS: Patient age ranged from 2 to 17 years (median: 10 years). Male: Female ratio 12:9. Aetiologies: congenital malformations $(\mathrm{n}=8)$, Os Odontoideum $(\mathrm{n}=2)$, trauma $(\mathrm{n}=8)$, osteomyelitis $(\mathrm{n}=1)$, idiopathic torticollis $(\mathrm{n}=1)$, Grisel's torticollis $(n=1)$. Ten (48\%) were myelopathic. Surgery was performed jointly by an adult neurosurgeon with specialist cervical expertise and a paediatric neurosurgeon. Procedures included: Anterior PEG screw $(n=2)$; Combined anterior-posterior approaches: Transoral resection of the odontoid peg after prior $\mathrm{C} 0-\mathrm{C} 2$ fixation $(\mathrm{n}=1)$; Posterior approaches: $\mathrm{C} 0-2$ fixation $(\mathrm{n}=4), \mathrm{C} 0-\mathrm{C} 2$ fixation and decompression $(n=4), C 1-C 2$ fixation $(n=8)$ and C1-C2 fixation and decompression $(n=2)$. No intra-operative complications. One post-operative complication occurred with a post-operative haematoma requiring surgical evacuation following a C1-2 fixation and decompression. This patient had an associated deterioration in their neurological symptoms. Mortality rate was $0 \%$. Follow-up completed in 20 out of 21 patients. Twenty patients (95\%) had radiological improvement post-operatively. Thirteen symptomatically improved, six remained symptomatically unchanged, two had new neck pain, one patient was neurologically worse. One patient with new neck pain underwent removal of the occipital rods, with pain resolving following this.
CONCLUSION: Instrumented fusion at the craniovertebral junction is a viable method to stabilise pathology occurring at this complex biomechanical junction. Neurological function can be maintained and improved as evidenced in this series and in literature.

Keywords: Craniovertebral junction, Craniocervical, Instability, Fixation, Fusion

\section{PF-060}

\section{Spine \\ Retrospective evaluation of occipito-cervical junction craniometric modifications after clival chordoma sur- gery in children}

Lelio Guida, Sandro Benichi, Christian Saint Rose, Syril James, Michel Zerah, Stéphanie Puget, Kevin Beccaria Department of Pediatric Neurosurgery, Hôpital Necker, Assistance Publique Hôpitaux de Paris, Paris, France, Université de Paris

OBJECTIVE: Clival chordoma may result in spinal instability due to infiltration of occipito-cervical joints and ligaments. Surgical treatment can have further consequences on regional stability, but its impact on a growing spine has never been assessed so far. We analyzed modifications of the occipito-cervical junction (OCJ) in a pediatric population affected by clival chordomas and attempted to find predictive factors associated to postoperative instability.

MATERIAL-METHODS: A retrospective study included children affected by clival chordomas diagnosed from 2005 to 2019 at Necker Hospital, Paris, France. Craniovertebral craniometric angles and measures (Welcher's basal angle, odontoid retroversion, Klaus' index, tentorial angle and pBC2 line) were analyzed on preoperative, 3-months, 1-, 5- and 10-years postoperative MRIs. An age-homogeneous cohort of patients with lesions not involving the OCJ was analyzed as a control group.

RESULTS: 15 patients were enrolled in the analysis (mean age \pm SD $9.4 \pm 3.6$ years, range 2.3-15.9 years, male/female ratio: 4/11). Most common location was lower clivus (confined or cervical spine-extended forms) $(\mathrm{n}=10)$. Occipitocervical ligament invasion was found in 10 patients, and condyle invasion in 5 patients. Two patients needed occipito-cervical fixation, while 1 patient underwent suboccipital decompression for basilar invagination. Significant changes of the Klaus'index and the tentorial angle were found at 5 years $(37.43 \pm 4,85$ vs $31.25 \pm 6.21, \mathrm{p}=0.044)$. Patients with lower clivus tumors had a different evolution depending on the age at diagnosis: children less than $10 \mathrm{y}-\mathrm{o}$ showed significant 3-months postoperative modifications, but later restored original craniometric values. Patients older than 
$10 \mathrm{y}-\mathrm{o}$ displayed progressive changes of the craniometric indexes during long-term follow-up $(34.29 \pm 1.45 \mathrm{vs}$ $26.84 \pm 3.88, \mathrm{p}=0,045)$.

CONCLUSION: Clival chordomas treatment can result in significant changes in the complex biomechanics of the OCJ. These changes are influenced by tumor localization and might be balanced by the growth potential in younger children.

Keywords: Chordoma, occipito-cervical junction, spinal instability, craniometry, occipito-cervical fusion, sub occipital decompression

\section{PF-061}

\section{Spine}

Atlanto-axial rotatory subluxation: Do clinical features and radiological appearance predict treatment modality?

Welege Samantha Buddhika Wimalachandra, Iaoannis Mavridis, Ashish Basnet, Joshua Pepper, Katie Herbert, Richard Anthony Walsh, Desiderio Rodrigues, William B Lo, Guirish A Solanki

Department of Neurosurgery, Birmingham Children's Hospital, Birmingham, United Kingdom

BACKGROUND: Diagnosis and management of torticollis due to Atlanto-axial rotatory subluxation (AARS) in children requires a clinico-radiological approach. Currently there is no consensus on management based on clinical or imaging features alone. In our unit, AARS is managed based on clinical severity and the radiological findings in a stepwise approach from collar application with muscle relaxation, to manipulation under anaesthesia, halo immobilization and surgical correction with fixation.

OBJECTIVE: We aim to correlate stratified clinico-radiological factors with specific management interventions and outcome.

MATERIAL-METHODS: 12-year retrospective review. All cases of torticollis with AARS confirmed on cervical CT. The demographics, symptom duration, Fielding and Hawkins (FH) AARS types and treatment received were reviewed. Treatment interventions were also evaluated at two-time groups (2007-2014 and 2015 current) to look for a change of practice.

RESULTS: Thirty-eight children presented with torticollis. Age ranged from 6 weeks to 14.7 (median of 7.9) years, $\mathrm{F}: \mathrm{M}=2.36$.

Etiology: Trauma 18(47\%), Infection 9(24\%), spontaneous 7(19\%), Iatrogenic 3(8\%). 36(95\%) were FH Type I-II. Two spontaneous late presentations with grade III-IV went on to halo immobilization and corrective surgery. For FH type I-II prior to 2014, 7 halos were applied in 18 cases initially against only 2 in 20 cases, 2015 onwards ( $\mathrm{p}=0.0364$ ). Collar alone or manipulation with collar application sufficed for $18 / 20(90 \%)$ in this group. Symptom duration was not associated with invasive immobilization of either halo or surgical fixation ( $p=0.058)$. Resolution of AARS with non-invasive treatment (collar alone or MUA+collar) was associated with FH type I and II combined ( $\mathrm{p}=0.044)$.

CONCLUSION: We present a large contemporary AARS series categorized radiologically (Fielding-Hawkins classification). We confirm that in up to $90 \%$ of cases Collar and muscle relaxation is highly effective. Halo application/ surgical intervention is reserved for FH III-IV or failures.

Keywords: atlanto-axial rotatory subluxation, torticollis, Fielding-Hawkins, halo, collar

\section{PF-062}

\section{Spine \\ Split laminotomy in pediatric spinal surgery}

Yoko Nakanishi, Ryoko Umaba, Noritsugu Kunihiro, Yasuhiro Matsusaka, Hiroaki Sakamoto

Department of Pediatric Neurosurgery, Osaka City General Hospital, Osaka, Japan

OBJECTIVE: We have performed split laminotomy in pediatric spinal surgery to preserve the normal architecture of the spine. We report the method and outcome of split laminotomy.

MATERIAL-METHODS: Data obtained in patients who underwent split laminotomy at Osaka City General Hospital between January 2009 and December 2018 were reviewed retrospectively. Split laminotomy is performed using a scissor or sagittal saw without exposing the muscles attached to the spinous process and the lamina, and then spreading the spinous process and the lamina by retractors. Postoperative spinal deformity was evaluated by clinical symptoms and pre- and postoperative MRI.

RESULTS: Split laminotomy was performed on 110 patients under the age of 15 (93 occult spina bifida, 15 spinal cord tumors, 1 subpial lipoma, 1 arachnoid cyst). Median age at surgery was 7 months (0-173 months). The number of spilt laminae were 1 in 28 cases, 2 in 52 cases, 3 in 16 cases, 4 in 5 cases, 5 in 5 cases, 10 or more in 4 cases. In all 110 cases, it was possible to secure a surgical field for excision or removal of the lesion. Dural tear occured in one case of 11-year-old spinal lipoma but injury to nervous structures was never observed. Spinal deformities were evaluated in 76 patients who had undergone MRI more than one year after surgery. Postoperative spinal deformities revealed scoliosis in two cases of occult spina bifida, but these may have the possibility of re-tethering. In a case of spinal subpial lipoma 
in which 13 vertebral arches were split, kyphosis appeared, but did not affect daily life.

CONCLUSION: In children compared to adults, the vertebral arch is elastic, and the traction of the spinous processes can expand a wider surgical field. Split laminotomy in pediatric spinal surgery seemed to be a safe, simple, and useful method.

Keywords: split laminotomy, pediatric spine, spinal deformity

\section{PF-063}

\section{Vascular}

\section{Pediatric AVM in children}

Alexandru Szathmari ${ }^{1}$, Motaz Al Sereihi ${ }^{1}$, Rel Gerald Boukaka $^{1}$, Djene Ibrahima Kaba ${ }^{1}$, Pierre Aurelien Beuriat ${ }^{1}$, Federico Di Rocco ${ }^{2}$, Francis Turjman ${ }^{3}$, Carmine Mottolese ${ }^{1}$ ${ }^{1}$ Departement of Pediatric Neurosurgery. Child Mother Hospital. Hospices Civils de Lyon, Lyon, France

${ }^{2}$ Departement of Pediatric Neurosurgery. Child Mother Hospital. Hospices Civils de Lyon, Lyon, France. Claude Bernard University, Lyon, France

${ }^{3}$ Neurointerventional radiology. "Pierre Wertheimer Neurological and Neurosurgical Hospital. Hospices Civils de Lyon, France. Claude Bernard University, Lyon, France

OBJECTIVE: Arteriovenous malformations represent a major cause of morbidity and mortality in children. Despite the advancements in neurosurgery, the best modality of treatment, whether embolization, surgery, radiosurgery or a combination of the three, is still discussed. We retrospectively reviewed our series of 72 children focusing on those treated recently from 2011 to 2018.

MATERIAL-METHODS: From 72 patients managed in our institution since 1990, 26 patients (11 boys and 15 girls) with an average of 9.6 years old (range 2 months to 17.4 years; median 9.5) were operated between 2011 and 2018. Criteria of evaluation were the post-operative neurological status improvement (according to the MRC scale and the Rankin score) and the degree of resection on the post-operative delayed angiography. One patient was admitted electively following an incomplete resection in another institution. The rest were admitted in the emergency department for acute presentations, of which 15 presented with hemorrhage. All had headaches in the initial setting with various localizations. One child presented with coma. A neurological deficit was found in 6 children, while 5 patients developed seizures. All patients were submitted to an angiography. Six patients were embolized in emergency setting. One of them underwent urgent surgical resection immediately afterwards. Twelve patients benefited from a combined treatment of staged presurgical embolization followed by surgical resection. Eight of the remaining patients were treated with endovascular embolization alone.

RESULTS: The follow-up was at least 1 year. All but one child with complex extensive intra-extra-cranial fistula are alive. None of the children needed further radiosurgery for residual lesions. At last follow-up 2 patients presented with residual motor deficits. Twenty-four patients have normal schooling or work, while 2 patients need assistance.

CONCLUSION: Our strategy for treatment of AVM in the last years associating embolization and microsurgical removal allowed obtaining good results in term of mortality and morbidity.

Keywords: AVM in children, staged presurgical embolization, microsurgery

\section{PF-064}

\section{Vascular \\ Long-term outcome of indirect bypass for young children with Moyamoya disease}

Seung Ki Kim ${ }^{1}$, Eun Jim $\mathrm{Ha}^{1}$, Byung Kyu Cho ${ }^{3}$, Kyu Chang Wang $^{4}$, Ji Hoon Phi ${ }^{1}$, Ji Yeoun Lee ${ }^{1}$, Kyung Hyun Kim ${ }^{2}$, Eun Jung $\mathrm{Koh}^{2}$

${ }^{1}$ Department of Neurosurgery, Seoul National University Hospital

${ }^{2}$ Division of Pediatric Neurosurgery, Seoul National University Children's Hospital

${ }^{3}$ Department of Neurosugery, Aremd Forces Capital Hospital

${ }^{4}$ Neuro-oncology Clinic, National Cancer Center

BACKGROUND AND AIM:The prognosis of moyamoya disease (MMD) in young children is known to be worse than that of older patients. However, there are few studies on children at this age. The aim of this study is to evaluate the long-term outcomes of indirect bypass surgery on young children with MMD.

METHODS:A total of 1,417 MMD children underwent indirect bypass surgery from August 1988 to October 2020. This study included 78 patients who were less than 3 years at the time of surgery, excluding patients with moyamoya syndrome. The clinical feature of MMD patients under the age of 3 were assessed. In addition, we analysed the longterm clinical outcome of 58 children who were followed up for more than 5 years. The mean clinical follow-up duration was $165 \pm 76$ months (range, 65-332 months). Crosssectional analysis was performed to evaluate overall clinical outcomes based on Lansky Play Performance scale (LPS). To analyse the longitudinal effect of surgery, the annual risk of symptomatic infarction or haemorrhage after indirect 
bypass surgery was calculated with a person-year method, and the event-free survival rate was evaluated using the Kaplan-Meier method.

RESULTS: The overall clinical outcome was favourable (LPS $>70$ ) in $82.8 \%$ of the patients. Overall perioperative adverse event rate was $14.1 \%$ (including 1 death due to severe brain swelling after infarction). During follow up period, there were 2 symptomatic infarction on the operated hemisphere. All symptomatic infarctions were occurred within 5 years after surgery. There was no haemorrhagic event. At last follow up, $82.9 \%$ of patients became seizurefree. Annual infarction rate is $0.25 \%$ per-person year. The 10 -year event-free survival rates for infarction ipsilateral to the operated hemispheres were $96.6 \%$.

CONCLUSIONS:Indirect bypass surgery could provide satisfactory long-term improvement in clinical outcome and prevention of recurrent infarction in young children with MMD.

Keywords: moyamoya disease, stroke, child

\section{Flash Abstracts}

\section{FL-001}

\section{Antenatal diagnosis and treatment \\ Pronostic Criterias for Spina Bifida aperta based on MyeLDM concept}

Timothée De Saint Denis ${ }^{1}$, Catherine Garel ${ }^{2}$, Paul Maurice ${ }^{3}$, Federico Di Rocco ${ }^{4}$, Stéphanie Friszer ${ }^{3}$, Shushanik Hovhannisyan $^{5}$, Jean Marie Jouannic ${ }^{3}$, Michel Zerah ${ }^{1}$

${ }^{1}$ Department of Pediatric Neurosurgery, Necker University Hospital, Paris, France

${ }^{2}$ Department of Radiology, Armand Trousseau Hospital, Paris, France

${ }^{3}$ Department of Fetal Medecine, Armand Trousseau Hospital, Paris, France.

${ }^{4}$ Department of Pediatric neurosurgery, CHU de Lyon HCL, Bron, France

${ }^{5}$ Department of Neonatalogy, Necker University Hospital, Paris, France

OBJECTIVE: Spinal dysraphisms are classically separated in open or closed. In our in utero myelomeningocele (MMC) repair program (PRIUM) we observed a recurrent pitfall diagnosis with LDM. Progress in diagnosis made us consider some dysraphisms as intermediate. We proposed to name them MyeLDM. More than a name we tried to review each signs that help us to give an accurate prognosis.

MATERIAL-METHODS: From 2013 to 2020, 133 cases were referred to our program. 104 were MMC, 12 were LDM. We reviewed 17 cases of MyeLDM operated postnatally. Antenatal diagnosis, cutaneous aspect, anatomic spinal features, cerebral indirect signs of open dysraphism and other associated malformations were evaluated.

RESULTS: We identified three anatomical factors that help us to evaluate the functional prognosis: opening, Spinal cord dysplasia and traction on the tethered site. The opening is assessed on observation and imaging by describing the skin coverage and /or by amniocentesis, posterior fossa modification (Chiari II) and hydrocephalus. Spinal dysplasia varies from a real placode to a single spotted termination of the spinal cord. Mechanical traction is also considered as a possible neurological worsening factor, partially reversible after surgery.

CONCLUSION: Considering Spina Bifida Aperta malformation with the MyeLDM spectrum offers us a tailored evaluation of the prognosis. This evaluation has three major anatomical criterias: degree of opening, extension of neural tube defect and traction intensity. These three factors associated with the level of the malformation give a range of neuro-urologic, motor and cognitive more precise prognosis.

Keywords: Spina Bifida, antenatal, Myelomeningocele, LDM

FL-002

\section{Dysraphism}

Is limited dorsal myeloschisis truly a benign form of spinal dysraphism??? -A series of 19 cases at tertiary level hospital

Shashank Ravindra Ramdurg, Anita Mahanta, Anuradha Patil

Mahadevappa Rampure Medical College, Kalaburagi, Karnataka, India

OBJECTIVE: Limited dermal myeloschisis (LDM) is a distinctive spinal dysraphic condition characterized by a fibroneural stalk tethering to the underlying spinal cord and an external globular or flat lesion. Objective of the paper was to study the epidemiological, clinical, radiological, therapeutic and prognostic aspects of this less known condition. MATERIAL-METHODS: All patients of operated limited dermal myeloschisis from 2012 January to 2019 November were evaluated and followed up. Data was collected from patient files, discharge summaries, operative notes and death records.

RESULTS: 19 cases of LDM presented to the department. 17 patients underwent definitive surgery. Median age was 4.84 months(range 1-12 months, excluding patient of 50 years). Male to female ratio was 10:9. Lesions were classified as saccular or flat variety. There were 12 saccular and 7 flat lesions. Commonest location was dorsal spine-11 cases 
followed by 4 each in cervical and lumbar region. Associations were: syrinx -6 cases, chiari malformation- 3 cases, 1 case each of meningocoele, atretic encephalocoele, dipolmyelia, diastamatomyelia and filar lipoma. Presentation was in the form of lesion at the back in all cases. All patients underwent exploration of lesion and detethering. Biopsy was characterized by a fibro-neural/vascular stalk(Figs. 1-4).

Follow-up ranged from 6 months to 6 years. One patient of dorsal saccular variety developed hydrocephalus, one developed retethering for which he was re explored while another case of wound infection with CSF leak was managed conservatively. Rest of the patients had an uneventful recovery. A neglected case of LDM in 50 year lady developed ulcerative squamous cell carcinoma with metastases.

CONCLUSION: LDM's are a distinctive clinicopathologic spinal dysraphic condition. They are benign only in their presentation as evidenced by large number of cases with associated syrinx. Though most have good results post surgery, one needs to be vigilant about development of hydrocephalus, retethering and rarely development of malignancies in chronically untreated lesions.

Keywords: Limited Dorsal Myeloschisis, spinal dysraphism, syrinx, hydrocephalus, retethering

\section{FL-003}

\section{Dysraphism \\ Immediate \& long-term surgical outcome as well as pos- sible risk factors for delayed Detoriation in patients of LIPOMENINGOCELE in a tertiary care center}

\section{Dhruvkumar Hiralal Baru, Jaimin K Shah, Shailendra J \\ Solanki \\ Department of Neurosurgery, Gujarat University, Ahmedabad,India}

OBJECTIVE: To review clinical presentation, surgical outcome, post op complications \& long term follow up in patients of lipomeningocele.

MATERIAL-METHODS: Data from records of 56 children with Lipomeningocele from January 2014-March 2020 were included. Their clinical presentation, timing of surgery, procedure done, post op complications and neurological status in pre \& immediate post op \& at follow up were studied. Their functional outcome \& risk factors for delayed detoriation at long term follow up were studied.MRI of concerned part with whole spine screening with brain screening was done pre operatively \& at follow up.

RESULTS: 26 patients presented in 1st year of life, median age-4 months. All of these were asymptomatic, except lump over back \& had normal neurology. The remaining were in age group of 1.5 -18 years, median age -9 years
\& presented with Urinary disturbances,walking difficulty, stool incontinence \& backache in descending order. All the patients were considered for surgery at their earliest irrespective of their symptomatology. Near total/total excision of lipoma was done in 42 patients, of which 4 had early detoriation of neurology which recovered on follow up at 3 months. At median follow up of 12 months, (3-36 months), of 30 patients in symptomatic group, 18 patients improved, 10 remained static while 2 had detoriation of neurology. While in asymptomatic group of 26 children, 25 continued to remain same while 1 had detoriation of neurology.Delayed detoriation was found to be at 15 months (12-36 months). All 14 patients who underwent partial excision, developed recurrence and required Re-surgery, while in other group of 42 , only 3 developed reccurence.

CONCLUSION: Earliest and total/near total excision of lipoma with division of filum terminale should be th goal of surgery. Delayed intervention \& partial excision of lipoma are risk factors for delayed neurological detoriation.

Keywords: Lipomeningocele, Tethered cord, Conus lipoma, Complex spinal lipoma, Lumbosacral lipoma, Spinal dysraphism

\section{FL-004}

\section{Dysraphism \\ Urological outcome following surgery for primary teth- ered cord syndrome}

\section{Navneet Singla}

Postgraduate Institute of Medical Education and Research, Chandigarh, INDIA

OBJECTIVE: Primary Tethered Cord Syndrome (TCS) is a congenital disorder due to defect in central neurulation and usually presents with pain, sensory and motor deficits, urological symptoms, foot deformities, scoliosis, cutaneous stigmata. The main aim of surgical detethering is to release the tethering structure and the chronic tension on the cord. Urological recovery and good outcome depends upon preoperative deficits and timing of surgery.The Main objective of this study is to analyze the urological outcome at 6 months post-operative period after detethering for Primary TCS and its association with type of anomaly.

MATERIAL-METHODS: A total of 48 patients of Primary TCS with age ranging from 2 to 12 years were taken. They were assessed with pre-operative symptoms, USG KUB and urodynamic study( UDS) and then re assessed at 6 month post-operative.

RESULTS: Out of 48 patients, 26 (54\%) had pre operative urological symptoms. 38 patients showed abnormal UDS. 29 (60\%) patients had improvement in UDS score while $17 \%$ 
showed worsening of UDS score. Younger patients and patients having pre operative urological symptoms improved more. Patients with LMMC having Neural Placode did not show significant benefit.

CONCLUSION: Early age of presentation and patients without Neural placode show better urological outcome.Urodynamic studies should be considered as one of the first line investigation in evaluation of patients of TCS.

Keywords: Tethered Cord Syndrome, Urodynamic Study

\section{FL-005}

\section{Dysraphism}

Surgical management of myelomeningocele-related spinal deformities

Olga M Sergeenko, Sergey O Ryabykh, Alexander V Burtsev, Alexander V Gubin

Neurosurgical departments, Ilizarov Center, Kurgan, Russia

OBJECTIVE: To evaluate optimal timing and type of surgical treatment of myelomeningocele-related spinal deformities and to provide long term follow-up of surgical treatment. MATERIAL-METHODS: We reviewed and presented clinical pictures, treatment strategies and results of 30 patients with myelomeningocele-related spinal deformities, treated at the Ilizarov Center (Kurgan, Russia) from 2010 to 2018. RESULTS: Average age was 7.0 years. Average preoperative neurological status according to a modified JOA Scale (mJOA) was 7.6 points (JOA Scale, Benzel's modification). Average functional status was 48.9 points according to a Functional Independent Measure scale (FIM). 11 patients had lordoscoliosis (lordosis $71.8^{\circ}$, scoliosis $62.9^{\circ}$ average Cobb angles), 15 patients had kyphoscoliosis (kyphosis $72.5^{\circ}$, scoliosis $34.4^{\circ}$ average Cobb angles) and 4 patients had pathologic kyphosis only ( $89.8^{\circ}$ average Cobb angles). Average duration of surgery was $215.8 \mathrm{~min}$. Average total blood loss was $188.5 \mathrm{ml}$. Average angle of kyphosis correction was $61^{\circ}$, scoliosis correction $25^{\circ}$ and lordosis correction $25^{\circ}$ (Cobb angles). Average duration of hospitalization was 15.9 days. Average follow-up was 24.8 months. Total number of complications was 21 . Reoperations were needed in 10 cases. Neurological status, according to the mJOA scale improved 0.6 points in average. Functional status, according to the FIM, increased 6.6 points in average.

CONCLUSION: Early surgical correction of myelomeningocele-related spinal deformities improves body balance and quality of life. A dual growing rod technique is safe and effective in cases of moderate neuromuscular spinal deformities in early age. Kyphectomy is a challenging procedure with high complication rates, especially skin problems, but in cases of heavy rigid kyphosis it has no alternatives.
Keywords: myelomeningocele-related spinal deformities, thetered spinal cord

\section{FL-006}

\section{Dysraphism}

Monitoring profile and significant event criteria for Intraoperative neuromonitoring in detethering of tethered cord at the level of conus medullaris

Suhas Udayakumaran $^{1}$, Sri Valli Puthi ${ }^{2}$, Siby Gopinath ${ }^{2}$

${ }^{1}$ Division of Paediatric Neurosurgery, Amrita University, Kochi, India

${ }^{2}$ Division of Electrophysiology, Amrita University, Kochi, India

OBJECTIVE: To define the significant event criteria based on the typical IONM profile in surgery for the tethered cord (TCS) at the level of conus medullaris of the spinal cord.

MATERIAL-METHODS: The study includes prospectively collected data from one hundred eighty-seven consecutive patients who underwent detethering of TCS under Intraoperative neuromonitoring (IONM), from January 2011 to February 2020. The preoperative neurological and urological status was compared with the postoperative status. Under GA, the following parameters viz.amplitude, latency and duration of the waveforms in the baseline TcMEP was recorded. The events were considered significant if the IONM checklist is satisfied and if 50\% change in amplitude and $10 \mathrm{~ms}$ change in latency of the waveforms when compared with the baseline potentials. The postoperative neurological status was recorded immediately after surgery and at six months.

RESULTS: We had 22 electrophysiological events in the 187 patients who underwent detethering. Of which $81 \%$ $(n=18)$ showed improvement of conduction parameters in the monitored nerve and $18 \%(\mathrm{n}=4)$ showed deterioration of the parameters.

The events showing improvement had an increase in amplitude (Average 40\%), decrease in latency (Av. $12 \mathrm{~ms}$ ), and increase in duration (Av.15 ms) when compared to baseline TcMEP. The events showing deterioration had a reduction in amplitude (Av.45\%), increase in latency (Av.15 ms), and decrease in duration (Av.17 ms) when compared to baseline TcMEP.

Totally there were 6 patient (including 2 false negatives) with clinical worsening (Motor and bladder together).

The positive predictive value was $100 \%$, with the negative predictive value of $97.3 \%$. The diagnostic accuracy was $96.1 \%$.

CONCLUSION: Amplitude, change in latency and duration of the waveform are essential parameters useful in indicating a change in conducting physiology of a monitored nerve 
during detethering. 50\% change in amplitude from baseline MEP is found to be a reasonably sensitive value to identify a change in conducting physiology of the nerve.

Keywords: Intraoperative neuromonitoring, Tethered cord syndrome, Spinal dyraphism

\section{FL-007}

\section{Dysraphism}

Low Rates Of Ventriculo-peritoneal Shunt Insertion After Post-natal MMC Closure: A Single Institutional Experience

Phong Nguyen ${ }^{1}$, Laura Hobart Porter ${ }^{2}$, Rebecca Watkins Bregy $^{3}$, Eylem Ocal ${ }^{1}$

${ }^{1}$ Division of Pediatric Neurosurgery, Arkansas Children's Hospital / Department of Neurosurgery, University Of Arkansas For Medical Sciences, Little Rock, Arkansas- USA ${ }^{2}$ Division of Pediatric Rehabilitative Medicine, Arkansas Children's Hospital / University Of Arkansas For Medical Sciences-Little rock, Arkansas, USA

${ }^{3}$ Arkansas Children's Hospital, Little Rock, AR

OBJECTIVE: The rate of hydrocephalus requiring treatment with ventriculo-peritoneal shunt (VPS) placement after post-natal closure of myelomeningocele (MMC) is classically known to be about $80 \%-90 \%$. The management of Myelomeningocele Study (MOMS) showed that the inutero MMC closure has decreased the rate of hydrocephalus, therefore the need for CSF diversion, to $40 \%$. However, in-utero closure is not the standard of care and is only performed at a number of institutions in the USA. We evaluated the rate of VPS placement in MMC patients at our institution where only post-natal closure is performed. The criteria and the algorithm to identify the need for VPS placement were presented.

MATERIAL-METHODS: A retrospective evaluation of MMC patients from April 2011 to April 2020 was conducted. We identified 81 patients with MMC. Demographics, initial and follow-up imaging results, head circumference measurements were collected. The presence of clinical signs and symptoms of hydrocephalus were documented. A simple clinical algorithm and thresholds for treatment were developed. The recently published guidelines of Spina Bifida Care Association (SBCA) were also incorporated in our care.

RESULTS: We identified 81 patients with MMC. 47 of 81 patients required VPS placement. The rate of VPS placement in our institution by applying strict clinical criteria for treatment was $58 \%$. This number is evidently less than the $80 \%$ value classically cited in the literature and is comparable to the institutions which report low VPS rates.
CONCLUSION: Optimizing CSF dynamics and minimizing the VPS related adverse outcomes is one of the goals in the care of MMC patients. One way of minimizing shunt related complications is to follow a strict clinical criteria to decrease the rate of VPS placement. The criteria followed by Arkansas Children's Hospital Spina Bifida team using clinically determined factors have decreased the number of shunts in MMC patients below the traditionally accepted rates.

Keywords: Myelomeningocele, post-natal closure, shunt, hydrocephalus

\section{FL-008}

\section{Epilepsy}

Drug and substance abuse in refractory epilepsy

Raafat Abdeldayem

Toxicology dept, mansoura university, egypt

OBJECTIVE: Seizures often occur in substance abusers. The present work aimed to study the etiology of nonresponse to antiepileptic drugs by estimating their serum levels and screening of drugs and substance abuse in patients with resistant epilepsy.

MATERIAL-METHODS: This study was conducted in epilepsy outpatient clinic, 924 patients with intractable epilepsy were included. They subjected to.

-Toxicology screen for detection of drug and substances abuse by analysis of urine and blood samples.

-Measurements of the level of antiepileptic drugs in the blood.

All assays run on the system use of EMIT (Enzyme Multiplied Immunoassay Test) and confirmed by GC/MS (gas Chromatography/Mass Spectrum).

RESULTS: Confirmed Positive results for drugs and substances abuse were detected in 246 of 924 patients (26.62\%) by GC/MS. Cannabis was the first abused drug (29.27\%). Only 17 patients show serum level of antiepileptic drugs within therapeutic range, but 169 patients' levels were below it and 60 patients with levels above it.

CONCLUSION: Substances abuse may be the cause of resistant epilepsy as they are epileptogenic by themselves or due to drug-drug interaction with the antiepileptic.

Keywords: urine samples; blood samples; EMIT; GC / MS.

\section{FL-009}

\section{Epilepsy}

Seizure outcome after surgery for pediatric epilepsyrelated brain tumor 
Kenichi Usami ${ }^{1}$, Keita Terashima ${ }^{2}$, Chikako Kiyotani ${ }^{2}$, Yuichi $\mathrm{Abe}^{3}$, Hideki Ogiwara ${ }^{1}$

${ }^{1}$ Division of Neurosurgery, National Center for Child Health and Development

${ }^{2}$ Division of Neuro-Oncology, National Center for Child Health and Development

${ }^{3}$ Division of Neurology, National Center for Child Health and Development

OBJECTIVE: The aims of this study are to report clinical characteristics and outcome of pediatric epilepsy-related brain tumor (ERBT) and to discuss treatment strategy.

MATERIAL-METHODS: Children less than 18 years old who underwent surgery for ERBT were retrospectively analyzed. Patients in whom epilepsy had been controlled before surgery were excluded.

RESULTS: Twenty-one children ( 8 boys and 13 girls) were analyzed in this study. The mean age at surgery was 6.8 years. Tumor was astrocytic tumor in 10 , gangliogioma in 4 and dysembryoplastic neuroepithelial tumor in 3. Intracranial subdural electrodes were placed prior to tumor resection in 5 cases. Gross total resection (GTR) was achieved in $14(67 \%)$. Seizure free was achieved in $15(71.4 \%)$. GTR was significantly associated with seizure free $(p=0.002)$. CONCLUSION: Lesionectomy can achieve seizure free in most of ERBT, however, the resection of surrounding epileptic foci is required in some cases. Detailed examinations to detect the epileptic foci should be performed in ERBT, particularly in case of drug-resistant intractable epilepsy.

Keywords: epilepsy, brain tumor, seizure outcome

\section{FL-010}

\section{Epilepsy \\ Epilepsy And Focal Cortical Dysplasia. Should The Two- Medication Failure Paradigm Be Pursued?}

\author{
Ido Ben Zvi ${ }^{1}$, Noelle Enright ${ }^{2}$, Christin Eltze ${ }^{2}$, Helen Cross $^{2}$, \\ Zubair Tahir $^{1}$, Martin Tisdall ${ }^{1}$ \\ ${ }^{1}$ Paediatric Neurosurgery, Great Ormond Street Hospital for \\ Children \\ ${ }^{2}$ Paediatric Neurology, Great Ormond Street Hospital for \\ Children
}

OBJECTIVE: Focal cortical dysplasia (FCD) is a malformation of cortical development and is highly associated with pharmacoresistant focal epilepsy. Standard indications for epilepsy surgery in patients with FCD include drug resistance (failure of 2 adequately tolerated and appropriately dosed anti-epileptic agents). Given the high incidence of drug resistant epilepsy in these children, this delay may not be warranted. The aim of the study was to see if these patients should be operated at an earlier stage.

MATERIAL-METHODS: The medical records of more than 150 children presenting to the north London epilepsy network with epilepsy and a presumed FCD were retrospectively reviewed. Seizure outcome post-surgery was calculated using the Engel scale at 12-month follow-up.

RESULTS: $85.8 \%$ of patients referred to our centre underwent epilepsy surgery, $5 \%$ were offered surgery but declined and $8 \%$ were well controlled on medication. $1.2 \%$ are awaiting surgery. Of those who underwent surgery $53 \%$ had an Engel score of 1 at 1 year postoperatively. The number of years from diagnosis to surgery was not associated with improved outcome, neither was the number of medications the child had been on prior to surgery, the age at seizure onset, the type of surgery required, the need for stereo EEG (SEEG), nor the histology after surgery. Concordance between magnetic resonance imaging (MRI) and ictal EEG was strongly associated with Engel score of 1 at one year follow up $(\mathrm{p}=0.0004)$.

CONCLUSION: The vast majority of this patient group will have epilepsy surgery. The main outcome predictor is correlation between MRI findings and ictal EEG localization. This suggests that patients with FCD and epilepsy whose EEG localization is concordant with MRI findings should be considered as epilepsy surgery candidates earlier in their course. This could improve their quality of life and cognitive function, and reduce burden on epilepsy services.

Keywords: Focal cortical dysplasia, Epilepsy, Seizure, Epilepsy surgery

\section{FL-011}

\section{Epilepsy \\ intraoperative ultrasound in epilepsy surgery caused by focal cortical dysplasia}

$\underline{\text { Salavat Valentinovich Mirkhaydarov }}^{1}$, Albert Akramovich Sufianov $^{2}$

${ }^{1}$ Federal State Autonomous Educational Institution of Higher Education I.M. Sechenov First Moscow State, Moscow, Russia

${ }^{2}$ Federal state-financed institution «FEDERAL CENTRE OF NEUROSURGERY» of ministry health of the Russia Federation (Tyumen), Tyumen, Russia

OBJECTIVE: determine the application of an intraoperative ultrasound (iUS) in epilepsy surgery caused by focal cortical dysplasia (FCD), highlight the advantages of iUS in comparing with another neurovisualisation and neuronavigation techniques. 
MATERIAL-METHODS: A retrospective study was analyzed a surgical treatment in 11 ( 9 male, 3 female) children with medical intractable epilepsy caused by histologically proved FCD at the department of pediatric neurosurgery of federal center of neurosurgery (Tyumen) from 2016 to 2018. 1 had FCD Ia $(9,1 \%), 1$ had FCD Ic $(9,1 \%), 2$ had FCD IIa $(18,2 \%), 7$ had FCD IIb $(63,6 \%)$. The age of patients was 6 months to 17 years old (mean age $10 \pm 6$ ).

All patients were operated with iUS. iUS was done with Flex Focus 800 ultrasound machine by BK Ultrasound. US transducers were High Frequency Linear 8870, Hockey Stick 8809, Craniotomy 8862.

During operation was detected location of FCD, completeness of removal of FCD.

RESULTS: IUS was performed to localize the lesion before opening the dura mater to make sure craniotomy wide enough and the lesion was fully exposed. Ultrasound characteristics, boundaries, and specific anatomical landmarks were acquired.

CONCLUSION: IUS provides real-time direct visualization of the explored area. It is widely available, cheap, not time consuming, give surgeon different planes which isn't available for intraoperative MRI. IUS completely safe for patient, compared to intraoperative CT. There is no need to use contrast for visualization of vessels surrounding the lesion, it is completely exclude side effects. This technique allows full evaluation of the lesion before dura opening and adds realtime information about anatomical landmarks, vascular and neural tissue. Moreover iUS can be fused with preoperative MRI or CT to increase resolution of images. It is dynamic methodic and allow to visualize structures any time during the operation. iUS can be used as a major technique of intraoperative neuronavigation.

Keywords: epilepsy, ultrasound, focal cortical dysplasia

\section{FL-012}

\section{Epilepsy \\ Epilepsy Surgery in Children Under Two Years of Age}

Joshua Pepper, William B Lo, Sunny Philip, Shakti Agrawal, Caroline Scott, Daren Martin Lane, Bryony Carr, Peter Bill, Buddhika Wimalachandra, Andrew Lawley, Stefano Seri, Anthony Richard Walsh

Epilepsy Surgery Service, Birmingham Children’s Hospital

OBJECTIVE: Medically intractable epilepsy in the very young represents a significant challenge to the epilepsy team and if untreated results in significant long term disability or death. Over the last few decades there has been an increased impetus to offer surgical intervention in younger children to improve not only long term seizure outcomes but neurocognitive and developmental milestones also. We report our data in children under two years of age, with medically intractable epilepsy who underwent surgery at our unit.

MATERIAL-METHODS: This is a retrospective study of all children under two years of age who underwent either resective or disconnection surgery for epilepsy at our unit. Seizure outcome was determined by the Engel Classification.

RESULTS: We performed 20 operations in 17 children. The average age at surgery was 1.0 years (standard deviation 0.6 ) with mean follow up of 2.9 years. The nature of the operation included hemispherotomy(11), lesionectomy(7) and lobar resection/disconnection(2). Underlying diagnosis includes hemimegaencephaly(5), focal cortical dysplasia(5), hemidysplasia(2), tuberous sclerosis(2) and other (3). Average operative time was $4-5 \mathrm{~h}$. At long term follow up three quarters (13/17) of patients were seizure free (Engel I), 2/17 had a worthwhile improvement (Engel III) and the remaining two children derived no benefit from surgery (Engel IV). There were no peri-operative deaths. Three children required second epilepsy surgery due to ongoing seizures. Surgery was abandoned in two cases due to operative blood loss. One child developed a pseudomeningocele. One child developed a cerebrospinal fluid leak and meningitis post operatively requiring return to theatre. No patient required a shunt. CONCLUSION: Epilepsy surgery in the very young results in excellent seizure outcome and can be considered a safe procedure.

Keywords: Epilepsy, very young, hemispherotomy, lesionectomy, disconnection

\section{FL-013}

\section{Epilepsy}

The use of robot-assistance enables more SEEG electrodes to be implanted and improves clinical outcome

Ioannis $\mathrm{N}$ Mavridis ${ }^{1}$, William $\mathrm{B}$ Lo $^{1}$, Buddhika Wimalachandra ${ }^{1}$, Sunny Philip ${ }^{2}$, Shakti Agrawal ${ }^{2}$, Caroline $\mathrm{Scott}^{3}$, Daren Martin Lamb ${ }^{3}$, Bryony $\mathrm{Carr}^{3}$, Peter Bill ${ }^{3}$, Andrew Lawley ${ }^{3}$, Stefano Seri ${ }^{3}$, Anthony Richard Walsh ${ }^{1}$

${ }^{1}$ Department of Neurosurgery, Birmingham Children's Hospital, Birmingham, West Midlands, England, United Kingdom

${ }^{2}$ Department of Neurology, Birmingham Children's Hospital, Birmingham, West Midlands, England, United Kingdom ${ }^{3}$ Department of Neurophysiology, Birmingham Children's Hospital, Birmingham, West Midlands, England, United Kingdom

OBJECTIVE: Since December 2016 we routinely performed stereoelectroencephalography (SEEG) procedures using the 
neuromate ${ }^{\circledR}$ robotic system (Renishaw plc). We aimed to study whether the use of robot was associated with a change of practice, surgical parameters and clinical outcomes, in particular seizure outcome and complications.

MATERIAL-METHODS: We retrospectively analyzed all SEEG cases of our department (December 2014-February 2020). Our sample consisted of 63 patients ( 24 boys and 39 girls), 4-18 years of age, who underwent 64 SEEG procedures. The neuromate ${ }^{\circledR}$ robotic system was used in 48 cases. The surgical parameters studied included the laterality of implantation (unilateral or bilateral), side of implantation (right or left), number of implanted electrodes (side-based and total), duration of recording (time from insertion until explantation of electrodes), and surgical complications. We also studied clinical outcomes of the first 32 patients (non-robot-assisted: 16; robot-assisted: 16), of which 23 proceeded to resective epilepsy surgery, using the Engel classification for outcome.

RESULTS: The mean number of implanted electrodes in robot-assisted cases was significantly higher than non-robotassisted ones ( 12.6 vs 7.6 electrodes, $p<0.0001$ ). The complication rate was the same between the two groups, namely $6.3 \%$. There was no difference between the non-robot and robot-assisted groups in terms of laterality of implantation, duration of recording and the proportion of patients proceeding to further resective epilepsy surgery (non-robot: 12/16, robot-assisted: 11/16, with one awaiting surgery). However, robot-assistance increased remarkably the proportion of SEEG patients who were seizure free (Engel class IA) at 12 months from $31.3 \%(5 / 16)$ to $56.3 \%(9 / 16)$ $(\chi 2=2.032, p=0.154)$.

CONCLUSION: The use of the neuromate ${ }^{\circledR}$ robotic system enables more SEEG electrodes to be implanted without increasing surgical complications in children and it is associated with improved epilepsy surgery outcome.

Keywords: clinical outcome, complications, Engel classification, epilepsy surgery, robot-assistance, SEEG electrodes

\section{FL-014}

\section{Epilepsy}

Language development after multilobar surgery of the posterior cortex in infants and young children with refractory epilepsy

Tomonori Ono $^{1}$, Ryoko Honda ${ }^{1}$, Yoshiaki Watanabe ${ }^{1}$, Shiro $\mathrm{Baba}^{2}$, Keisuke Toda ${ }^{3}$, Hiroshi Baba ${ }^{4}$.

${ }^{1}$ Epilepsy Center, National Nagasaki Medical Center, Omura, Japan

${ }^{2}$ Department of Neurosurgery, Nagasaki University School of Medicine, Nagasaki, Japan
${ }^{3}$ Department of Neurosurgery, National Nagasaki Kawatana Medical Center, Kawatana, Japan

${ }^{4}$ Epilepsy Center, Nishi-isahaya Hospital, Isahaya, Japan

OBJECTIVE: Cognitive decline, including language and intellectual impairments, is frequently associated with childhood refractory epilepsy. Resective or disconnective surgery (RDS) is utilized for seizures and may also have a significant influence on cognitive outcomes. However, with respect to multilobar surgeries, a concern exists that more brain functions may be lost following this more extensive surgery. The posterior cortex is most often the target of multilobar surgeries, however postoperative cognitive outcomes have not been investigated in detail.

MATERIAL-METHODS: We retrospectively reviewed the results for 20 children who underwent multilobar RDS of the posterior cortex and were followed for greater than 12 months after surgery. Among the 11 patients with lesions identified by MRI, RDS was an initial option. In the remaining 9 patients, stepwise procedures of callosotomy and RDS were applied due to lateralized features of the seizures and EEG after callosotomy. Age at initial surgery ranged 8-88 months, and follow-up ranged 12-71 months.

RESULTS: Nine patients remained seizure-free throughout the entire follow-up period. Developmental outcomes were assessed, using developmental quotient (DQ) scores, in 18 patients, including motor, language, and social subscales. Receptive language DQ significantly improved in seven patients and expressive language DQ significantly improved in seven patients. No patients experienced significant improvement in physical motor or manipulation DQ. Postoperative DQ gain was significantly higher in receptive language and social relationship DQ than in physical motor and manipulation DQ. These outcomes were independent of age at surgery, duration of illness, and postoperative seizure outcomes.

CONCLUSION: Extensive RDS of the posterior cortex had a more positive influence on receptive language than did motor development. This may occur because such language functions were formed in, or translocated to, the contralateral, healthier hemisphere during the course of the development of epilepsy. Our data also suggest that extensive surgery is beneficial for language development.

Keywords: Epilepsy surgery, Cognitive outcome, Language development, Children

\section{FL-015}

\section{Epilepsy}

Hemispherotomy for epilepsy in the very young 
Joshua Pepper ${ }^{1}$, William Lo ${ }^{1}$, Shakti Agrawal $^{2}$, Rana Mohammed $^{2}$, Jo Horton ${ }^{3}$, Selina Balloo ${ }^{3}$, Sunny Philip ${ }^{2}$, Ashish Basnat ${ }^{1}$, Welege Buddhkia ${ }^{1}$, Andrew Lawley ${ }^{2}$, Stefano Seri ${ }^{2}$, Anthony Richard Walsh ${ }^{1}$

${ }^{1}$ Department of Neurosurgery, Birmingham Children's Hospital, Birmingham, UK

${ }^{2}$ Department of Neurology, Birmingham Children's Hospital, Birmingham, UK

${ }^{3}$ Department of Neuropsychology, Birmingham Children's Hospital, Birmingham, UK

BACKGROUND AND AIM:Epilepsy is one of the most common neurological disorders in children. One third of very young children with epilepsy are resistant to medical treatment, which if left untreated can result in adverse outcome. While hemispherotomy is an established treatment its outcome is not that well reported in the very young child. We report the seizure and developmental results after hemispherotomy in children under three years of age.

METHODS:We reviewed a prospective database of all children under three years of age with medically intractable epilepsy who underwent hemispherotomy at our institution between 2012 and 2020. The demographics, epilepsy history, underlying etiology, operative and transfusion details, seizure and developmental outcome were analysed.

RESULTS: The mean age of seizure onset was 3 months (standard deviation [SD] 2.6 monthss) and age at surgery was 1.3 years (SD 0.77 years) with a mean follow up of 4 years. Diagnoses included hemimegaencephaly (5), hemidysplasia (2), hypoxic/hemorrhagic (2), traumatic (1), Sturge-Weber syndrome (1) and mild hemispheric structural abnormality with EEG/PET correlates (1). Eleven patients achieved Engel class I outcome and one achieved Engel IV at last follow up. No deaths, infections, cerebrovascular events or unexpected long-term neurological deficits were recorded. All children progressed neurodevelopmentally following surgery, but their developmental levels remained behind their chronological age with an overall mean composite vineland adaptive behaviour score of 57.72. One patient required insertion of a subdural peritoneal shunt, one patient required dural repair for a cerebrospinal fluid leak and one required aspiration of a pseudomeningocele. The operation was incomplete in two children due to blood loss, both of whom weighed under $5.7 \mathrm{~kg}$.

CONCLUSIONS:Hemispherotomy in children under the age of three offers excellent seizure control and an acceptable risk-to-benefit ratio in well-selected patients.

Keywords: hemispherotomy, epilepsy, pediatric
Intra-operative neurophysiological monitoring in selective dorsal rhizotomy

$\underline{\mathrm{Bo} \mathrm{Xiao}}^{1}$, Rong $\mathrm{Mei}^{2}$

${ }^{1}$ Department of Pediatric Neurosurgery, Shanghai Children's Hospital

${ }^{2}$ Department of Neurophysiology, Shanghai Children's Hospital

OBJECTIVE: The use of intra-operative neurophysiological monitoring (IONM) to guide selective dorsal rhizotomy (SDR) to treat spastic cerebral palsy (CP) has been practiced for decades. The word "selective" refers to the intra-operative selection and dissection of the afferent nerve rootlets in spinal cord which are considered to be involved more in spasticity, so as to achieve the purpose of reducing the muscle tone after the surgery. Since the importance and necessity of IONM in SDR have been questioned for years, the current review is trying to address those concerns via literature review.

MATERIAL-METHODS: In the current review we put our focus on history of IONM-guided SDR, evolving EMG interpretation methods and as well as their neuroelectrophysiological basis.

RESULTS: Taking a review of those EMG interpretation methods proposed over the past approximately 40 years, we could roughly categorize those methods into three major groups based on their basis of EMG interpretation patterns, with each being based on its own neurophysiological hypothesis. Though cases undergone these methods-guided SDR generally had good outcomes, it is still not clear which hypothesis fits more the neuroelectrophysiological nature in spinal cord of those spastic CP patients.

CONCLUSION: Selection of dorsal roots (rootlets) for rhizotomy in SDR is crucial, and directly correlated to the outcomes of CP cases which undergo such procedure. Giving the fact that those newly developed EMG interpretation methods over the past 20 years were mainly based on the clinical studies, their neuroelectrophysiological basis still remained unclear, though outcomes of $\mathrm{CP}$ patients undergone these methods-guided SDR were favorable, and surgery-related complications were extremely minimal. Nevertheless, IONM-guided SDR has provided an approach to better understandings of neuronal electrophysiological circuits in spinal cord in spastic CP patients, therefore giving the potential to optimize treatment.

Keywords: spasticity, selective dorsal rhizotomy, rootlet selection, neuroelectrophysiology, EMG interpretation

\section{FL-017}

\section{Functional}


Quality of life after selective dorsal rhizotomy: an assessment of family-reported outcomes using the CPQoL questionnaire

$\underline{\text { James MW Robins }}^{1}$, Isabel A Tulloch ${ }^{1}$, Abbey Boyle ${ }^{2}$, Kate Mccune $^{3}$, Rajib Lodh ${ }^{4}$, John R Goodden ${ }^{1}$

${ }^{1}$ Paediatric Neurosurgery, Leeds Children's Hospital, Leeds General Infirmary, Great George Street, Leeds LS1 3EX, UK ${ }^{2}$ University of Leeds Medical School, Leeds, UK

${ }^{3}$ Paediatric Physiotherapy, Leeds Children's Hospital, Leeds General Infirmary, Great George Street, Leeds LS1 3EX, UK ${ }^{4}$ Paediatric Neurorehabilitation, Leeds Children's Hospital, Leeds General Infirmary, Great George Street, Leeds LS1 3EX, UK

OBJECTIVE: Selective dorsal rhizotomy (SDR) is widely accepted as an effective procedure for management of lower limb spasticity in children with cerebral palsy. However, effects of the procedure on quality of life are not widely reported and less so using a structured and validated quality of life tool such as Cerebral Palsy Quality of Life Questionnaire (CPQoL).

Here, we present complete data for CPQoL outcomes for SDR patients operated in a single institution at 2 years follow-up.

MATERIAL-METHODS: Patients were operated over a 5 -year period by the same surgeon using the same technique in a single institution. CPQoL questionnaires were completed by patients and families pre-operatively and at 6 months, 1 year and 2 years postoperatively.

Data was collected prospectively.

RESULTS: A total of 78 patients (58 male, 20 female), age range 2.6-13.8 years (median 6.33) were included whom underwent SDR between October 2012-November 2017. All had complete follow-up up to 2 years post-procedure (most recent November 2019). Four patients were excluded due to incomplete follow-up data.

Statistically significant improvement was seen across five out of seven CPQoL domains and this was sustained to 2 years post-SDR.

CONCLUSION: We demonstrate using a validated Quality of Life Tool that SDR has a beneficial effect on the quality of life for patients with cerebral palsy at this length of follow-up.

Keywords: selective dorsal rhizotomy, spasticity, cerebral palsy, outcomes, quality of life

\section{FL-018}

\section{Functional}

\section{A Ten-Year Retrospective Review of Intrathecal Baclofen Delivery in Children at a Single Centre}

Neeraj Kalra, Isabel Tulloch, Sharron Peacock, Katie Davis, Andie Mulkeen, Rajib Lodh, John Goodden

Leeds Children's Hospital, Leeds, United Kingdom

OBJECTIVE: We review our experience of delivering the Gamma-aminobutyric acid agonist Baclofen intrathecally over the past ten years to manage the symptoms of children who have spasticity.

MATERIAL-METHODS: We performed a retrospective analysis reviewing the outcome data for consecutive children who underwent procedures involving intrathecal Baclofen delivery at a single centre between January 2010 and January 2020. Data was collected on patient demographics, the intervention performed and treatment outcomes.

RESULTS: In total, 80 patients underwent Baclofen-related procedures (including test doses and/or pump insertions and/ or revisions) with 71 test dose procedures being completed and 48 primary intrathecal Baclofen pumps being inserted. In total 160 procedures were completed on these patients (including surgeries to treat subsequent surgical complications). In the patients undergoing primary Baclofen pump insertion, mean patient age was 10 years (median age 9.4 years, range 3.9 to 16.7 years). The male: female ratio for pump insertion was 32 males: 16 females.

Of the primary pump insertion cohort, 6 patients (12.5\%) experienced post-operative infections requiring surgical intervention, 9 patients (18.8\%) experienced leak of cerebrospinal fluid at the catheter insertion site requiring further surgery and within the remaining cohort 3 patients (6.3\%) required pump/catheter revision within six months of primary surgery.

CONCLUSION: We have evaluated the experience of a single centre in performing procedures relating to intrathecal Baclofen delivery. We discuss the most frequent complications within this subset of patients and ways in which we have found to minimise these adverse events to optimise the patient care pathway.

Keywords: Intrathecal, Baclofen, Gamma-aminobutyric acid agonist, Spasticity, Pump, Catheter

\section{FL-019}

\section{Functional}

Selective Dorsal Rhizotomy: Goals of a new "0 Level" or "interlaminar approach" less invasive technique

Syril James, Sandro Benichi, Edouard Mazerand Department of Pediatric Neurosurgery, Necker-Enfants Malades Hospital, AP-HP and Paris Descartes University OBJECTIVE: The objective of this study was to describe a new less invasive surgical technique performed in France at the Necker Enfants Malades Hospital. The secondary aim 
was to analyze the short-term effects of this technique and eventually show the advantages regarding the previous ones. MATERIAL-METHODS: We used a retrospective case series analysis to compare 3 patients who underwent a Selective Dorsal Rhizotomy with a new "zero level" technique without laminectomy, to 5 matched controls who had undergone SDR with the multi-level technique or the single-level technique.

RESULTS: Postoperative pain and alongside the intake of analgesics are limited in time in the less invasive surgical technique, if compared to the other techniques. The values of the Cobb angle from the 1st week and the 3rd month after surgery were matched: children operated with the less invasive technique tended to remain stable over time.

CONCLUSION: SDR is nowadays a safe and useful intervention for targeted patients identified by strict criteria. This procedure effectively reduces spasticity associated with cerebral palsy by selective sectioning of the lumbosacral afferent nerve rootlets. The developing of an increasingly less invasive technique, the "0 level "allows the surgeon to decrease the length of intervention, supposing that time spent in working on a tiny space, after having acquired experience, is finally recuperated in not practicing laminoplasty. Patients experiment decreased post-operative pain and spine degeneration in time. Both surgical and rehabilitation professionals can benefit from a shorter hospitalization period, bringing also psychological benefits to families. Last but not the least the reduction of the dimensions of the scar encourage self-acceptance.

Keywords: Selective dorsal rhizotomy, 0 level surgery, spasticity, cerebral palsy

\section{FL-020}

\section{Functional}

Could pediatric cerebral palsy population with spasm in rectus femoris benefit from the procedure of our newly modified rhizotomy protocol guided single-level approach SDR?

Qijia Zhan ${ }^{1}$, Min Shen ${ }^{2}$, Wenbin Jiang ${ }^{1}$, Xidan $\mathrm{Yu}^{2}$, Chunxin $\mathrm{Xu}^{2}$, Shuyun Jiang ${ }^{3}$, Junlu Wang ${ }^{1}$, Rong $\mathrm{Mei}^{1}$, Bo Xiao ${ }^{1}$

${ }^{1}$ Department of Neurosurgery, Affiliated Shanghai Children's Hospital, Shanghai Jiaotong University, Shanghai, China

${ }^{2}$ The Disabled Rehabilitation Vocation Center of Shanghai, Shanghai, China

${ }^{3}$ Gait and Motion Analysis Center, Yueyang Hospital of Integrated Traditional and Western Medicine, Shanghai University of Traditional Chinese Medicine, Shanghai, China
OBJECTIVE: The spasticity of rectus femoris in pediatric cases with cerebral palsy (CP) decreased in our newly modified rhizotomy protocol guided single-level approach SDR (SL-SDR) procedure, though we exclude rectus femoris as one of the target muscles in our SL-SDR setting. Our aim is to find out how the spasticity of rectus femoris in pediatric $\mathrm{CP}$ cases decreased in SL-SDR procedure.

MATERIAL-METHODS: In the current study, we retrospectively conducted a cohort review of cases younger than 18 years of age diagnosed with spastic $\mathrm{CP}$ cases with muscle tone of rectus femoris which were $\geq 2$ grade (Modified Ashworth Scale) pre-op who undergone novel protocol guiding SL-SDR in the Department of Neurosurgery, Shanghai Children's Hospital since Jul. 2018 to Dec. 2019.

RESULTS: A total of 21 eligible cases were included in our study. Thirty-eight rectus femoris which was $\geq 2$ grade were identified during pre-OP assessment. The mean muscle tone of them was $2.1 \pm 0.3$ pre-OP. Based on our protocol, there were 70 rootlets evoked EMG response associated with rectus femoris $(48,9$, and 13 in target muscle of hip adductor, hamstring, gastrocnemius, respectively). Mean 2.2 weeks After SDR, there were 28 of 38 recuts femoris with the muscle tone decreasing by a mean of 1.3 degree in our cases $(p<0.05)$. In the meanwhile, the mean EMG amplitude of them was $\geq 100 \mu \mathrm{V}$ during the surgery. Kinematics of joints of knee and surface EMG of rectus femoris on the affected side in our cases demonstrated a major improvement during their gait cycles. No permanent surgery-related complications were recorded in the current study.

CONCLUSION: Our modified rhizotomy protocol guided SL-SDR can effectively decrease muscle tone of rectus femoris which were $\geq 2$ grade pre-op. Pediatric $\mathrm{CP}$ cases with spasm in rectus femoris benefit from the procedure of singlelevel SDR when guided by our novel rhizotomy protocol.

Keywords: novel rhizotomy scheme, spasm of rectus femoris, EMG responses interpretation, outcome measures

\section{FL-021}

\section{Germ Cell Tumors}

A Single UK Centre Analysis of Open Surgical Resection for Pineal Region Tumours in Children

Oliver Richards, Saira Nisar, Chloe Gelder, John Goodden, Paul Chumas, Atul Tyagi

Department of Neurosurgery, Leeds General Infirmary, Leeds, United Kingdom

OBJECTIVE: To ascertain if a supratentorial or infratentorial approach improved surgical resection or increased 
post-operative complications in the surgical treatment of pineal region tumours.

MATERIAL-METHODS: The relevant patient demographics, laboratory tests and data on ophthalmic deficits were collected for patients undergoing surgical intervention at a United Kingdom regional neurosurgical centre with the diagnosis of a pineal region tumour, between 2008-2018. Radiological data, including the pre- and post-operative resection volumes, anatomical relation to the deep venous structures and the location of residual tumour was also analysed. Patients who underwent biopsy only were excluded. Results were compared for patients who were treated with infratentorial or supratentorial/transtentorial approaches. Unpaired $\mathrm{t}$-test and chi-squared tests were used as appropriate.

RESULTS: Ten patients were identified. Three underwent endoscopic third ventriculostomy and biopsy only. The remaining seven underwent open surgical debulking; five through a supratentorial approach, two through an infratentorial approach. Five patients presented with gross ophthalmological signs that did not improve in four of the patients. There was no significant association between the choice of approach and these postoperative deficits or complications $(p=0.43)$. An average extent of resection of $91.3 \%$ (Range $15.7-100 \%)$ was achieved. There was no significant difference between the surgical approach and extent of resection $(p=0.09)$. There was no significant relationship between the deep venous structures (Internal Cerebral Vein and Vein of Galen) and the choice of approach. The supratentorial approach often left residual tumour within the infratentorial compartment.

CONCLUSION: This small, single centre study, whilst not demonstrating any statistically significant findings, has produced some interesting observations. Our unit tends to favour a supratentorial approach to these lesions, and there is no evidence of an increased risk of ophthalmic problems with doing so. Our resection volumes are comparable between approaches. However, each approach does risk leaving tumour residuum in predictable locations.

Keywords: pineal, extent of resection, complications, residuum

\section{FL-022}

\section{Global Pediatric Neurosurgery}

Scope of pediatric neurosurgery as a subspeciality in a neurosurgical referral centre of a low income country

Sagar Koirala, Suresh Bishokarma, Pratyush Shrestha Department of Neurosurgery, Upendra Devkota Memorial National Institute of Neurological and Allied Sciences, Kathmandu University, Kathmandu, Nepal
OBJECTIVE: The field of neurosurgery is expanding rapidly in Nepal with more than 70 neurosurgeons registered in the Nepalese Society of Neurosurgeons. Subspecialization in different field is the next step to go about. So, in this study we have looked into various pediatric neurosurgeries performed in a tertiary centre done over the past 10 years to see if pediatric neurosurgery is a viable neurosurgical subspeciality in today's context.

MATERIAL-METHODS: A retrospective study was performed at a tertiary level neurosurgical referral centre. All pediatric cases of age less than 18 years who were operated over 10 years period were included. Data was collected from operation lists and patients discharge summaries. Data were tabulated in Microsoft Excel and analyzed.

RESULTS: There were 601 surgeries performed. Male to female ratio was almost $2: !$. The youngest kid operated was 2 days old. Operative cases ranged from cranial and spinal trauma, hydrocephalus, posterior fossa tumors, sellar/suprasellar tumors, lobar mass, pineal tumors, brain abscess, vascular malformation, $\mathrm{CVJ}$ anomalies, spinal dysraphysm etc. CONCLUSION: There is enough variety as well as number of pediatric cases coming to a neurosurgical referral centre for surgery. Along with development of other subspeciality in neurosurgery, pediatric neurosurgery has an equal prospect as almost $40 \%$ of the population of Nepal ins under 18 years.

Keywords: pediatric neurosurgery, scope, subspeciality, low-income country

\section{FL-023}

\section{Global Pediatric Neurosurgery \\ Policy Recommendations for the Prevention of Spina Bifida in Low-and-Middle Income Countries}

Ashley K. Birch ${ }^{1}$, Jacob R. Lepard ${ }^{2}$, Jean Wilguens Lartigue $^{3}$, Jacquelyn Corley ${ }^{4}$, Kee B. Park ${ }^{3}$, Benjamin $C$. Warf $^{l}$

${ }^{1}$ Department of Neurosurgery, Boston Childrens Hospital, Boston, MA, USA

${ }^{2}$ Department of Neurosurgery, University of Alabama at Birmingham, Birmingham, AL, USA

${ }^{3}$ Program in Global Surgery and Social Change, Department of Global Health and Social Medicine, Harvard Medical School, Boston, MA, USA

${ }^{4}$ Department of Neurosurgery, Duke University Medical Center, Durham, NC, USA

OBJECTIVE: Neural tube defects (NTD) occur in approximately 300,000 neonates worldwide annually and are a leading cause of death, illness, and disability. Increasing momentum surrounding the adoption of National Surgical 
Obstetric and Anesthesia Plans (NSOAPs) necessitates practical roadmaps to better inform policy-makers in how best to address neurosurgical disease.

MATERIAL-METHODS: A literature review was completed by searching databases for the key words of neural tube defects, prevention, folate, and folic acid to accumulate all available scientific evidence regarding the prevention of spina bifida. Recommendations were constructed using the NSOAP framework and reviewed by leaders in the fields of neurosurgery and global health.

RESULTS: Only one third of the world's flour from industrial mills is fortified with folic acid, amounting to approximately $18 \%$ of NTD that are immediately preventable. Based upon this, it is recommended that countries immediately take measures to fortify processed wheat and flour products. Women of child-bearing age should also supplement with folic acid. More prevalent education regarding the importance of folate via online modules and printed materials is encouraged for healthcare workers. Close monitoring of geographic and demographic data is recommended to identify areas of greater incidence and prevalence. This will allow for more efficient use of preventative and therapeutic resources in high impact areas. The creation of laws and incentives will alleviate difficulty in non-profit and non-government organizations (NGO) entering the country and providing financial support for interventions.

CONCLUSION: The global burden of neurosurgical disease is too extensive to be addressed entirely from an operating theatre and greater policy level changes are needed to provide neurosurgical care delivery in an equitable fashion. This is a broad system issue in which nurses, surgeons, clinicians, public health workers, policy makers, and parents should become content experts and evidence-based advocates involved in finding ways to reduce this burden by making prevention a priority.

Keywords: Spina Bifida, Folic Acid, Global Health, Prevention Strategies

\section{FL-024}

\section{Global Pediatric Neurosurgery \\ Clinical characteristics and prognostic factors of intrac- ranial ependymoma in infants}

\section{Yu Jianzhong, Li Hao}

Department of Neurosurgery, Children's Hospital of Fudan University,Shanghai, China

OBJECTIVE: This study aims to identify the features and potential prognostic factors of intracranial ependymoma in infants.
MATERIAL-METHODS: Medical records of patients who were surgically treated between June 2008 and June 2019 and pathologically confirmed as intracranial ependymoma below 3 years old were collected from the Children's Hospital of Fudan University. Multiple statistical analyses were performed to identify potential prognostic factors, including Kaplan-Meier survival analysis using SPSS Statistics 19.0 software, log-rank test for analyzing the significance of the difference, and Cox regression multivariate analysis.

RESULTS: A total of 38 patients were investigated. Among them, 21 were male and 17 were female. There were 5 cases under 1 year old, 22 cases of 1-2 years old, and 11 cases of $2-3$ years old. 7 cases (18.4\%) were Grade I-II and 31 cases (81.6\%) were Grade III. 11 cases $(29.7 \%)$ were supratentorial and 26 cases $(70.3 \%)$ were infratentorial. The most common clinical symptoms served as the initial clinical manifestation of patients were intracranial hypertension symptoms, followed by neurological dysfunction symptoms. The median time from symptom onset to initial visit was 7 days. $32(84.2 \%)$ cases underwent total tumor resection and 6 $(15.8 \%)$ cases underwent subtotal resection. 14 patients received adjuvant therapy after surgery (radiotherapy and/ or chemotherapy). 10 patients had recurrence after surgery. Univariate analysis revealed that overall survival (OS) was affected by patients' age, the extent of surgical resection, presence or absence of postoperative adjuvant therapy, and the presence or absence of neurological dysfunction. Multivariate analysis showed that patients' age and surgical resection extent were independent factors associated with OS. CONCLUSION: Infratentorial ependymoma had higher incidence than supratentorial ones. Younger patients had poorer prognosis. Patients with neurological dysfunction symptoms before surgery had poorer prognosis. The surgical resection extent was positively correlated with the prognosis. Postoperative adjuvant therapy improved the prognosis.

Keywords: Infants, Ependymoma, Surgery, Adjuvant therapy, Prognostic factors

\section{FL-025}

\section{Global Pediatric Neurosurgery \\ Predictors of Delayed Access to Care for Pediatric Hydrocephalus in Vietnam}

Le Quang $\mathrm{My}^{1}$, Jacob Richard Lepard ${ }^{2}$, Tran Van $\mathrm{Si}^{3}$, Dang Do Thanh $\mathrm{Can}^{1}$, Cau Vu Hung ${ }^{3}$, Le Nam Thang ${ }^{3}$, James M Johnston $^{2}$, Brandon G Rocque ${ }^{2}$, Benjamin C Warf ${ }^{4}$

${ }^{1}$ Neurosurgical Department, Children's Hospital \#2, Ho Chi Minh City, Vietnam

${ }^{2}$ Department of Neurological Surgery, University of Alabama at Birmingham, Birmingham, Alabama, USA 
${ }^{3}$ Neurosurgical Department, National Hospital of Pediatrics, Hanoi, Vietnam

${ }^{4}$ Department of Neurological Surgery, Boston Children's Hospital, Boston, Massachusetts, USA

OBJECTIVE: Access to care and early referral are important components in timely treatment of pediatric hydrocephalus. The current study seeks evaluate for socio-economic predictors of late presentation with increased head size for newly diagnosed pediatric hydrocephalus in Vietnam.

MATERIAL-METHODS: All new patients presenting to Children's Hospital 2 in Ho Chi Minh City or the National Hospital of Pediatrics in Hanoi, Vietnam with diagnosis of hydrocephalus were included. Head circumferences and ages were used to create $\mathrm{z}$-scores of head size at presentation. Data from the General Statistics Office of Vietnam were used to assign socio-economic variables to each patient based upon their home province. Simple and multiple linear regression were used to find statistically significant predictors of increased z-score at presentation.

RESULTS: A total of 925 patients were included with $62.2 \%$ males (575/925) and mean age of 11.5 months (SD 20.4). Etiology of hydrocephalus was primarily non-postinfectious hydrocephalus $(77.4 \%, 716 / 925)$, followed by post-infectious hydrocephalus $(12.1 \%, 112 / 925)$, and myelomeningocele $(10.5 \%, 97 / 925)$. Multivariate linear regression demonstrated that the percentage of population living below the poverty line in the patient's home province was associated with increased $\mathrm{z}$-score at presentation $(\mathrm{p}=0.003)$. Additionally, the average female age at marriage was inversely correlated with z-score at presentation, with younger average age at first marriage being associated with increased z-score $(\mathrm{p}=0.038)$.

CONCLUSION: Increased understanding of socio-economic predictors of poor access to pediatric neurosurgical care will allow for more targeted and strategic interventions such as local public education and improved referral networks.

Keywords: Hydrocephalus; Vietnam; Global neurosurgery; access to care; poverty

\section{FL-026}

\section{Global Pediatric Neurosurgery}

First UK series of endoscopic strip craniectomy with post-operative helmeting for craniosynostosis: early report of peri-operative experience

Jonathan Halim $^{1}$, Curtis Budden ${ }^{3}$, Adikarige Silva ${ }^{2}$, Noor Ul Owase Jeelani ${ }^{2}$, David James Dunaway ${ }^{3}$, Juling Ong ${ }^{3}$, Greg James ${ }^{2}$

${ }^{1}$ Barts and the London School of Medicine, Queen Mary University of London, London, UK
${ }^{2}$ Department of Neurosurgery, Great Ormond Street Hospital, London, UK

${ }^{3}$ Craniofacial Unit, Great Ormond Street Hospital, London, UK

OBJECTIVE: Endoscopic strip craniectomy with postoperative helmeting $(\mathrm{ESCH})$ has emerged as a successful treatment for craniosynostosis, initially in North America. We report early outcomes from the first cohort of ESCH patients treated in the United Kingdom (UK).

MATERIAL-METHODS: Retrospective cohort study with electronic chart review.

RESULTS: 18 consecutive patients from first ESCH procedure in UK (May 2017) until end of 2019 were identified. The cohort consisted of 12 male and 6 female infants with craniosynostosis, with a mean age of 4.6 months (range 2.5-7.8 months) and weight of $6.8 \mathrm{~kg}$ (range 4.8-9.8 kg). The most commonly operated diagnosis was metopic synostosis $(n=8)$, followed by unicoronal $(n=7)$, sagittal $(n=2)$ and multisutural $(\mathrm{n}=1)$ synostoses. Median length of skin incision was $3 \mathrm{~cm}$ (range $2-10 \mathrm{~cm}$ ), with 15 patients having a single skin incision, 3 patients ( 2 sagittal/1 multisuture) having 2 incisions. 16/18 received no blood products, with 2 (both metopics) requiring transfusion (1 donor exposure). Mean operative time (including anaesthesia) was $96 \mathrm{~min}$ (range 40-127 min). The median length of hospital stay was 1 night, with no patient staying more than 2 nights (3 patients). 1 surgical complication was recorded, a suture abscess requiring oral antibiotics. All patients are currently undergoing helmet orthosis therapy and compliance so far has been $100 \%$. So far, no patients have required revisional surgery or squint surgery for strabismus.

CONCLUSION: Early peri-operative experience from the first UK cohort of ESCH suggests that this is a safe and well tolerated technique with low morbidity, need for transfusion and short hospital stay. Long-term results in terms of shape, cosmetic and developmental outcome are awaited and will be presented in due course.

Keywords: craniosynostosis, endoscopy, minimally invasive surgery, helmet orthosis

\section{FL-027}

Global Pediatric Neurosurgery

Surgical Systems Policy Recommendations for the Treatment of Hydrocephalus and Spina Bifida in Low and Middle Income Countries

Kathrin Zimmerman $^{1}$, Jacquelyn Corley ${ }^{2}$, Kee Park $^{3}$, John Mugamba $^{4}$

${ }^{1}$ School of Medicine, University of Alabama at Birmingham, AL, USA; Program in Global Surgery and Social Change, 
Department of Global Health and Social Medicine, Harvard Medical School, Boston MA, USA

${ }^{2}$ Department of Neurosurgery, Duke University Medical Center, Durham, NC, USA; Program in Global Surgery and Social Change, Department of Global Health and Social Medicine, Harvard Medical School, Boston MA, USA

${ }^{3}$ Program in Global Surgery and Social Change, Department of Global Health and Social Medicine, Harvard Medical School, Boston MA, USA

${ }^{4}$ Cure International

OBJECTIVE: Hydrocephalus and spina bifida are a major cause of morbidity and mortality worldwide and are among the most common neurosurgical disorders in children. As countries begin to adopt National Surgical Obstetric and Anesthesia Plans (NSOAPs), it is essential that pediatric neurosurgical conditions are included. The purpose of this study was to provide evidence-based policy recommendations for addressing pediatric neurosurgical disease.

MATERIAL-METHODS: A scoping review of the literature was conducted to compile and evaluate all scientific evidence regarding surgical systems and capacity required to treat hydrocephalus and spina bifida in the global context. Comprehensive recommendations were constructed using the NSOAP framework and reviewed by a panel of pediatric neurosurgery and policy experts. Recommendations were made in the following categories: infrastructure, workforce, service delivery, financing, information management, and governance.

RESULTS: We recommend that all referral hospitals as defined by the World Health Organization have the capacity to provide pediatric neurosurgical services. We recommend that there needs to be approximately one pediatric neurosurgeon per 52,000 children globally, based on current global burden of disease, and that surgical training centers are needed to meet these demands. We recommend that pediatric neurosurgery care be embedded into universal health coverage.

CONCLUSION: To address pediatric neurosurgical disease it is essential to advocate for evidence based surgical systems strengthening worldwide.

Keywords: hydrocephalus, pediatrics, spina bifida

\section{FL-028}

\section{Global Pediatric Neurosurgery \\ Minimal invasive cranioplasty in syndromic multisuture craniosynostosis}

Martina Messing Jünger, Friederike Knerlich Lukoschus, Andreas Röhrig
Neurochirurgische Abteilung, Asklepios Klinik, Sankt Augustin, Germany

OBJECTIVE: Early minimal invasive stripcraniectomies have become a standard procedure in single suture craniosynostoses, e.g. in sagittal synostosis. In order to prevent extreme deformities and complications related to increased ICP this technique can also be beneficial in syndromic multisuture craniosynostosis.

MATERIAL-METHODS: This is a retrospective study of a consecutive single unit series of patients who underwent minimal invasive stripcraniectomies in patients with syndromic multisuture craniosynostosis. Demographic and clinical data as well as surgical and follow up information have been investigated. The standard surgical procedure was excision of all cranial sutures (except the metopic suture) in all cases. The cosmetic results have been evaluated.

RESULTS: 8 patients have been operated so far. All of them were operated between week 4 and 12. The following syndromes have been treated: Apert (2), Muenke, Baere-Stevenson-cutis-gyrata, familiar TBC1D32-variant, Saethre-Chotzen, Mercedes-Benz- and Kleeblattschaedeldeformity of unknown genetic background (1 each). In all but one (Mercedes-Benz-deformity) patients, between 1 and 3 additional cranioplastic surgeries became necessary after 6 months of age. No surgical complications occurred. The child with Baere-Stevenson-cutis-gyrata-syndrome died at 2 years of age. He had tracheostoma due to choanal atresia since birth and vp-shunt. All others have no further complications, e.g. good eye status and no sleep apnea and are clinically stable (follow up $7-75$ months). The cosmetic results are good or very good after initial minimal invasive procedures.

CONCLUSION: Early minimal invasive stripcraniectomies are able to prevent extreme craniofacial deformities and complications related to increased ICP in syndromic multisuture craniosynostosis. The technique is safe, even in very young and compromised children and does not interfere with secondary surgical procedures.

Keywords: minimal invasive cranioplasty, multisuture craniosynostosis, syndromic craniosynostosis

\section{FL-029}

\section{Global Pediatric Neurosurgery \\ Clinical variety and prognosis of arachnoid cysts in children}

$\underline{\text { Stephanie Jünger }}{ }^{1}$, Friederike Knerlich Lukoschus ${ }^{2}$, Andreas Röhrig $^{2}$, Jasmin Al Hourani ${ }^{2}$, Sandra Kunze ${ }^{2}$, Martina Messing Jünger ${ }^{2}$ 
${ }^{1}$ Neurochirurgische Klinik, Universitätsklinik Köln, Köln, Germany

${ }^{2}$ Neurochirurgische Abteilung, Asklepios Klinik, Sankt Augustin, Germany

OBJECTIVE: Arachnoid cysts occur in different intracranial locations and the etiology is not yet fully understood. Management and prognosis are depending on the clinical presentation. Prenatal counseling on arachnoid cysts is becoming increasingly important and requires a profound knowledge regarding this entity.

MATERIAL-METHODS: This retrospective study of a consecutive single unit (5 surgeons) series of patients who underwent arachnoid cyst surgery between January 2010 and September 2019, provides demographic, clinical and imaging data and information about surgical treatment and outcome. Only symptomatic patients were operated. Statistics (SPSS, Version 25).

RESULTS: A total of 63 patients (71.4 male, 28.6 female) have been operated. Mean age was 50 months (range 0-191 months). Mean follow up was 40 months (0-121 months). 51(81\%) patients presented with symptoms/ signs of raised ICP. Focal neurological deficits were found in $16(30.2 \%)$, headache present in $9(14.3 \%)$ children. Galassi I-III cysts represent the predominant type $(19 ; 30.2 \%)$, followed by suprasellar $(10 ; 15.9 \%)$, quadrigeminal $(8 ; 12.7 \%)$, retrocerebellar, cerebellopontine angle and midline (each $7 ; 11.1 \%)$ and atypical $(5 ; 7.9 \%)$. Endoscopic and microsurgical fenestration have been performed in $17(27 \%)$ and $37(58.7 \%)$, additional insertion of a stent in $4(6.3 \%)$ or a secondary shunt in 5(7.9\%) cases. In $21(33.3 \%)$ patients one and in $8(12.7 \%)$ a second intervention became necessary. Complication/reintervention rate was $38 \%$. Reoperation rate was significantly higher in children $<1$ year of age $(\mathrm{p}=0.003)$. Cyst volume finally reduced in $85.7 \%$ and remained stable in $14.3 \% .70 \%$ of the patients were symptom free, $5 \%$ suffered from headache, $22 \%$ showed developmental disorders. All 7 midline cysts with corpus callosum agenesis (CCA) were male and showed developmental delay. All focal neurological symptoms resolved.

CONCLUSION: Arachnoid cysts occur in various intracranial locations. Complications and outcome are related to the location and the recurrence and revision rate is significantly higher in young infants. Midline cysts with CCA have a high chance of developmental disorders.

Keywords: arachnoid cyst, midline cysts with corpus callosum agenesis, CCA, Galassi

\section{FL-030}

\section{Global Pediatric Neurosurgery}

Brachytherapy in Paediatric Craniopharyngiomas: A Systematic Review and Meta-analysis of Recent Literature

Mariana M. Guimarães, Daniel D. Cardeal, Manoel J. Teixeira, José Erasmo D. C. Lucio, Felipe H. Sanders, Rodrigo K. Kuromoto, Hamilton Matushita

Department of Neurosurgery, University of São Paulo, São Paulo, Brazil

OBJECTIVE: Brachytherapy has been indicated as an alternative option for treating cystic craniopharyngiomas. Despite the difficulties regarding the complex nuclear infrastructure and consequent small number of brachytherapy studies, recent discoveries relating tumour characteristics to sensitivity to brachytherapy have stimulated this extensive systematic review and meta-analysis of the recent results aiming to summarise its efficacy and complications.

MATERIAL-METHODS: The systematic review was structured using PRISMA statements. The MEDLINE database was systematically reviewed from March 2010-February 2021 to identify qualified trials dealing with radioisotope brachytherapy in cystic craniopharyngiomas in the paediatric population, emphasising tumour control rates and complications.

RESULTS: A total of 228 individuals were analysed, of which 66 were children. The minimum average follow-up was five years. Considering the paediatric trials, partial and complete responses were achieved in $89 \%$ of patients with exclusively cystic lesions, compared to $58 \%$ in non-exclusively cystic lesions. The former observed progression in $3 \%$ of patients, while in the latter, $35 \%$ was reported. Visual and endocrine improvement reached $64 \%$ and $20 \%$, respectively, in the first group, in contrast to $48 \%$ and $7 \%$ in the second group, respectively. There were similar results considering the non-exclusive paediatric series, but with less expressive numbers.

CONCLUSION: These results reinforce the positive impact of radioisotope brachytherapy in the treatment of predominant monocystic or multicystic craniopharyngiomas, especially in the paediatric population, grounded by impressive tumour control rates, lower morbidities and single application in the majority of the cases. In the future, specific morphological tumour characteristics might be considered for a more assertive patient selection.

Keywords: craniopharyngioma, irradiation, brachytherapy, radioisotope

\section{FL-031}

Global Pediatric Neurosurgery 
Surgical treatment of isolated and syndromic craniosynostosis. Outcome in 96 consecutive cases

Oscar Guillermo García González ${ }^{1}$, Eduardo Morales Valencia $^{2}$, Carlos Morales Valencia ${ }^{3}$, Aarón Emanuel Serrano Padilla $^{4}$, Rubí Sotelo Serna ${ }^{4}$, Erick Moreno Pizarro ${ }^{4}$

${ }^{1}$ Department of Pediatric Neurosurgery,HospItal Regional de Alta Especialidad del Bajio,León,Guanajuato

${ }^{2}$ Universidad Nacional Autonoma de Mexico, León, Guanajuato

${ }^{3}$ Universidad Autónoma del Estado de México, Toluca, México

${ }^{4}$ Universidad de Guanajuato, León, México

OBJECTIVE: To review the results of the surgical treatment of craniosynostosis in 96 consecutive patients.

MATERIAL-METHODS: We retrospectively review. 96 cases of craniosynostosis were treated between August 2009 and March 2020. 14 syndromatic cases (7 Apert, 2 Crouzon, 5 Mercedes-Benz) 82 non-syndromic cases (35 sagittal, 19 coronal, 14 methopic, 3 lambdoid, 10 multiple sutures and 1 craneoestenoses shunt-related), The data associated with length of stay, blood loss, and operating times were reviewed. We also investigated the differences between endoscopically assisted suturectomy (EAS) versus open calvarial vault reconstruction (OCR).

RESULTS: The average age at the time of surgery was 8.9 months (range, 3 months to 3 years) with predominance in males (66\%). The surgical treatment consisted of 1) classic suturectomy ("bear skin") plus $360^{\mathrm{a}}$ remodeling in the sagittal cases, 2) fronto-orbital advancement plus frontal remodeling for unilateral or bilateral coronal cases, 3) parieto-occipital advance more parietal remodeling ("sunrise") and occipital ("barrel stave") in unilateral or bilateral lambdoid cases, 4) Combination of the above techniques in caes with affection of 2 or more sutures and 5) suturectomies assisted by endoscopy Some post-surgical complications occur (CSF leak and foreign body reaction to the suture and plates of polyglycolic acid; One transoperative death and another in the following weeks. No case of recurrence after suturectomy. The surgical duration, blood loss, days in ICU, and hospital length of stay were all significantly lower in the EAS group vs OCR (all $\mathrm{P}<0.05$ ).

CONCLUSION: Based on our experience, the classic surgical techniques allow an immediate correction to improve the volume of the cranial vault, as well as the resolution of the encephalic compression, with a very low percentage of complications. EAS offers a safe and effective treatment for sagittal craniosynostosis that is comparable in outcome to OCR, with no increase in morbidity and a shorter length of stay.

Keywords: Craniosynostosis, cranial vault reconstruction, bioabsorbable material, endoscopic assisted suturectomy
FL-032

Global Pediatric Neurosurgery

$A$ report on the separation of our 4th set of Craniopagus (CPT 4) twins at GOSH - are we learning from our experience?

Noor Ul Owase Jeelani ${ }^{1}$, David J Dunaway ${ }^{2}$, Juling Ong ${ }^{2}$, Gregory James ${ }^{1}$, Zubair Tahir ${ }^{1}$, Curtis Budden ${ }^{2}$, Silvia Schievano ${ }^{3}$, Richard Hayward ${ }^{1}$

${ }^{1}$ Department of Neurosurgery, Great Ormond Street Hospital, London, UK

${ }^{2}$ Department of Craniofacial Surgery, Great Ormond Street Hospital, London, UK

${ }^{3}$ FaceValue Research Team, University College London, London, UK

OBJECTIVE: We previously reported at the ISPN, our results for 3 sets of CPT twins separated at our institution between 2006 and 2019. Here we present our 4th set, undertaken in 2019-20, highlighting the surgical lessons learned and refined from our previous experiences.

MATERIAL-METHODS: A craniopagus pair of boys were referred to our charity, Gemini Untwined, at 13 months of age. They were of the O'Connell Total Vertical Configuration-type 2, similar to our most recent set, CPT-3.

The skin, bone and venous anatomy was similar to our previous case CPT-3; the dural defect and the brain interface was more extensive.

A logistical prompt, required us to change our technique. We undertook skin expansion first and then the neuro-vascular separation over 3 stages spanning 2 weeks. The total CPT- 4 separation process took 7 weeks. There was further refinement of our planning and surgical technique based on our previous experience.

RESULTS: Both twins sustained an infarct in their right parietal regions, as a result of ligation of the arterial cross over, venous congestion and separation of the fused brains. Three months post separation, Twin 1 has no obvious neurological deficit.

Twin 2 is making good, steady progress. He is able to sit with minimal support, uses both upper limbs purposefully, feeding and has a few words to his vocabulary.

CONCLUSION: Given our experience of 4 sets of CPT cases, with current technology, a multidisciplinary approach and staged surgical planning, it is possible to separate a significant proportion of CPT twins previously deemed un-separable. There is morbidity associated with these surgeries and it is for the medical and ethical community to decide on what level of neurological deficits are acceptable to achieve separation. Our charity, Gemini Untwined (www.gemin iuntwined.org), aims to collate a global repository of information on CPT cases to further these discussions. 
Keywords: Craniopagus, Global charity, Innovation, 3D modelling, virtual reality

\section{FL-033}

\section{Global Pediatric Neurosurgery \\ Incidence and Outcomes of Myelomeningocele in a ter- tiary referral hospital in the Philippines}

Juan Silvestre Grecia Pascual ${ }^{1}$, Reina Juno Uy Sumatra ${ }^{2}$, Mykha Marie Bajador Tabuzo ${ }^{2}$, Immanuel Villacorta Silverio $^{2}$, Edmund John Beltran Cayanong ${ }^{1}$, Ronnie Enriquez Baticulon ${ }^{1}$, Jacinto Blas Velez Mantaring Iii $^{3}$

${ }^{1}$ Division of Neurosurgery, Department of Neurosciences, College of Medicine and Philippine General Hospital, University of the Philippines Manila

${ }^{2}$ College of Medicine and Philippine General Hospital, University of the Philippines Manila.

${ }^{3}$ Division of Neonatology, Department of Pediatrics, College of Medicine and Philippine General Hospital, University of the Philippines Manila

BACKGROUND AND AIM:Myelomeningocele (MM) is a preventable spina bifida defect that disproportionately affects low-middle income countries like the Philippines. Despite multidisciplinary medical and surgical care, it still causes significant morbidity and mortality. This study aims to determine the incidence, management, and outcome of MM patients seen in the largest tertiary referral center in Manila, Philippines.

METHODS:A retrospective review of records was done among patients with MM treated at the Philippine General Hospital (PGH) from 2015-2019. Demographic and in-hospital care data were obtained. Outcomes considered were 30-day readmission for wound complications, 1-year readmission for shunt infection or malfunction, and mortality.

RESULTS: A total of 225 patients with MM were seen at the PGH from 2015-2019, 133 records were reviewed and included in the analysis. There was a slight male predominance (52\%), with $36 \%$ born in-hospital. Of the outside consults, $30 \%$ came from within the capital city. The most common location of MM was lumbosacral (66\%\%). Most frequent in-hospital on-board services were neurosurgery $(100 \%)$, pediatric neurology $(100 \%)$, and neonatology (38\%). Median time from birth or consult to repair was 6 days. Permanent CSF diversion was needed in 77\% and the most common type was ventriculoperitoneal shunt insertion (98\%). Median time from repair to CSF diversion was 15 days for all patients requiring this. Readmissions for wound complications, shunt infection and shunt malfunction occurred in 5\%,16\%, and $11 \%$, respectively. There were 7 mortalities with the most common cause being septic shock.
Pre-repair rupture of MM was significantly related to shunt infection on univariate analysis $(\mathrm{p}=0.011)$.

CONCLUSIONS:The experience in the PGH differs from established standards in MM care, particularly with regards to timing of surgery and on-board services. There is a need to identify in-hospital factors that affect care of MM patients in order to fully treat them.

Keywords: Myelomeningocele, spina bifida, CSF diversion, VPS

\section{FL-034}

\section{Hydrocephalus}

The movement of particles in cerebrospinal fluid is regulated by its size and molecular weight

Takuya Akai ${ }^{1}$, Toshihisa Hatta ${ }^{2}$, Shunsuke Yamamoto ${ }^{1}$, Satoshi Kuroda ${ }^{1}$

${ }^{1}$ Department of Neusosurgery, Toyama University, Toyama, Japan

${ }^{2}$ Department of Anatomy, Kanazawa Medical University, Kahoku, Japan

OBJECTIVE: Cerebrospinal fluid (CSF) may work as a key transporter of brain waste. Recently, new CSF drainage tracts were proposed; 1) cerebral interstitial tissue into capillary vessels, 2) meningeal lymphatic system, 3) nasal mucosal lymphatic system. These drainage ability will be decreased depend on age, and its correlation to dementia is suggested.

In this study, we investigated the extracranial outflow of particles in CSF. The analysis of CSF outflow mechanism will contribute for prevention of brain aging and cerebral degenerative disease, and treatment of hydrocephalus.

MATERIAL-METHODS: Mouse fetuses at a gestational age of 13 days were used. The fetuses enveloped by an amnion were exposed out of uterus. Fluorescent proves with differing molecular weights (MW) or gold particles with different size were injected into the lateral ventricle. The blood flow to fetus was preserved, and the movements of probes were monitored in live. In gold particle study, the fetuses were put back in dam for decided duration. And the fetuses were stained with silver, and transparented for observation. The lymphatic system was detected by immunostaining with LYVE-1 antibody.

RESULTS: 1) Fluorescent study: The injected probes dispersed into the 3 rd ventricle and aqueduct immediately, but did not move into the 4 th ventricle and spinal canal. With an injection of low and high molecular weight (LMW, HMW) probes conjugate, both probes dispersed into the brain, but only the LMW probe dispersed into the whole body. 2) Gold 
particle study: The accumulation of gold particles on the frontal skull base was observed. Only small size particle $(2 \mathrm{~nm})$ was found in liver at $15 \mathrm{~min}$ after injection.

CONCLUSION: The extracranial outflow of particles in CSF is regulated by its MW and size. The main channel may locate on the frontal skull base. The particles may drain into lymphatic system quickly.

Keywords: cerebrospinal fluid, outflow, channel, regulation, live image

\section{FL-035}

\section{Hydrocephalus}

Thrombocytopenia: an indicator for escaping posthemorrhagic hydrocephalus after intraventricular hemorrhage in neonates?

Ahmed El Damaty, Luca Giannone, Andreas Unterberg, Heidi Bächli

Heidelberg University Hospital

OBJECTIVE: Posthemorrhagic hydrocephalus (PHH) is a rare but serious complication among premature neonates, with consequences including mortality and severe neurodevelopmental disabilities. The causes of PHH are still not entirely understood, and its prevention and treatment are controversial. We tried to analyse the risk factors for such complication in our cohort.

MATERIAL-METHODS: We reviewed our neonatology data bank and included all patients who were born in the period from 1999-2014 with a gestational age below 28 weeks and suffered from an intraventricular hemorrhage (IVH) of any degree. We collected gestational age, gender, birth weight, IVH degree (I-IV), comorbidities, therapy measures, complications, period from first diagnosis PHH to implantation of a VP shunt, protein content of cerebrospinal fluid content before shunt implantation, clinical follow-up. All patients with a follow-up period of less than one year were excluded from the study.

RESULTS: We identified 180 patients, the cohort was divided into two subgroups, "B1" with 37 cases (patients with IVH + PHH) and "B2" with 143 cases (patients with IVH without $\mathrm{PHH}$ ). In group B1, $11 \%$ of patients had an IVH grade I, $19 \%$ IVH grade II and 70\% an IVH III. or IV. degrees. 19 patients were treated with a Rickham reservoir or EVD. A total of 19 shunts were implanted. 10 patients $(27 \%)$ die, only 1 patient showed thrombocytopenia. In subgroup B2, the majority of patients showed $51 \%$ IVH grade I, severe IVH grade III was present in $22 \% .25 .9 \%$ suffered from thrombocytopenia. The IVH grade showed strong significance (p-value: 0.0005). We found thrombocytopenia significantly higher in patients who did not develop PHH. (p-value: 0.002).

CONCLUSION: According to our results, thrombocytopenia could play a decisive role in avoiding development of $\mathrm{PHH}$ as a sequel of IVH. We recommend a randomized controlled trial to assess efficacy of antiplatelet drugs in avoiding $\mathrm{PHH}$ in this vulnerable group.

Keywords: Hydrocephalus, Posthemorrhagic

\section{FL-036}

\section{Hydrocephalus \\ Retrospective study of fetal ventriculomegaly; natural course and neurodevelopmental status}

\section{Farideh Nejat, Zohreh Habibi, Ghazaleh Kheiri}

Children's Medical Center, Tehran University of Medical Sciences

OBJECTIVE: Prenatal ventriculomegaly is diagnosed in 0.3 to $2 \%$ of foetuses based on lateral ventricular diameter in fetal ultrasound. It is classified as mild (10-12 mm), moderate $(12-15 \mathrm{~mm})$ and sever more than $15 \mathrm{~mm}$ which caused by perinatal infections, focal or generalized loss of brain volume, CSF pathway obstruction (leading to hydrocephalus), or some CNS malformations.

MATERIAL-METHODS: All pregnancies with mild and moderate ventriculomegaly referred to our clinic for consultation between 2010- 2018 were included in this study. We retrospectively collected demographic and ultrasonographic data and information on associated abnormalities. Telephone interviews were used to obtain information about pregnancy outcomes and long-term neurodevelopmental (CDC) followup and ventriculomegaly progression. All alive cases were followed for at least one year.

RESULTS: Among 121 cases of mild and moderate ventriculomegaly, 94 had complete information. The average gestational age at the time of diagnosis/counselling for the mild group was 7.3 and for the moderate group, 7.48 months. The mean age of children at the parental telephone interview was 34.04 months (range 12 to 110 months).

In follow up ultrasound scan, the width of ventricular dilation regressed in 5 cases, and all of the cases were from the moderate VM group. Ventricular width remained the same in 67 cases and progressed in 12 cases. $89.3 \%$ of mild group and $67.5 \%$ cases in the moderate group remained stable. 39 children had associated anomalies with 10 corpus callosum agenesis being the most frequent abnormality.

In this study, 6 pregnancies chose abortion, 7 cases died during neonatal time, and one of cases had stillbirth. In follow-up 59.8\% had normal neurodevelopment (mild $=73.3 \%$, 
moderate $=51.4), 16.1 \%$ had mild delay $(\operatorname{mild}=6.7 \%$, moderate $=24.3 \%$ ), $5.7 \%$ had moderate delay (mild $=0 \%$, moderate $=10.8 \%$ ), and $18.4 \%$ were diagnosed with severe delay $($ mild $=20 \%$, moderate $=13.5 \%, \mathrm{P}=0.024)$.

CONCLUSION: Children with fetal ventriculomegaly have a less risk of ventricular size progression and neurodevelopmental delay.

Keywords: fetal ventriculomegaly, ultrasound, neurodevelopment, progression

\section{FL-037}

\section{Hydrocephalus}

Initial outcomes of endoscopic third ventriculostomy with choroid plexus coagulation $(\mathrm{ETV}+\mathrm{CPC})$ in Vietnam

Tran Van $\mathrm{Si}^{1}$, Jacob Richard Lepard ${ }^{2}$, Cau Vu Hung ${ }^{3}$, Le Nam Thang ${ }^{1}$, James M Johnston ${ }^{2}$, Brandon G Rocque ${ }^{2}$, Benjamin C Warf ${ }^{4}$

${ }^{1}$ Neurosurgery Department, National Hospital of Pediatrics, Hanoi, Vietnam

${ }^{2}$ Department of Neurological Surgery, University of Alabama at Birmingham, Birmingham, Alabama, USA

${ }^{3}$ Neurology Department, National Hospital of Pediatrics, Hanoi, Vietnam

${ }^{4}$ Department of Neurosurgery, Boston Children's Hospital, Boston, Massachusetts, USA

OBJECTIVE: Pediatric hydrocephalus is a major cause of morbidity in low- and middle-income countries (LMICs). Endoscopic third ventriculostomy with choroid plexus coagulation (ETV + CPC) has been shown to be a viable treatment option with significant advantages unique to resource limited settings. However, to date, the majority of ETV + CPC outcomes data are limited to sub-Saharan Africa, and the utility of its application in other global regions therefore has not yet been studied.

MATERIAL-METHODS: All cases of endoscopic management of hydrocephalus at the National Hospital of Pediatrics in Hanoi, Vietnam were reviewed. All cases were done over a two-year period between January 2017 and December 2018. Demographics and clinical outcomes data were collected. Patients with less than 6 months follow-up were excluded.

RESULTS: A total of 155 patients underwent ETV or $\mathrm{ETV}+\mathrm{CPC}$ during the study period. The mean and median age at surgery was 2.6 and 1.1 years, respectively (range 0.6 months to 14.5 years). The most common etiologies of hydrocephalus were post-infectious $(44 / 155,28.4 \%)$, myelomeningocele $(4 / 155,2.6 \%)$, tumor $(13 / 155,8.4 \%)$, and congenital or other causes $(94 / 155,60.6 \%)$. Two patients had post-operative infections, and four cases were complicated by post-operative CSF leak. Treatment failure was noted in 47 patients $(30.3 \%)$ with a mean and median time to failure of 2.7 and 1.5 months, respectively. Of the failures, 12 underwent successful redo ETV and 35 received a ventriculoperitoneal shunt. Ultimately, of the 155 patients that underwent endoscopic management of their hydrocephalus, $121(78.1 \%)$ were successfully treated without need of shunting.

CONCLUSION: The initial data for outcomes of $\mathrm{ETV}+\mathrm{CPC}$ in Vietnam indicate very favorable results with the potential to alter the regional burden of shunt dependent hydrocephalus.

Keywords: Hydrocephalus; endoscopy; choroid plexus coagulation; global neurosurgery; post-infectious hydrocephalus

\section{FL-038}

\section{Hydrocephalus}

No difference in shunt survival between gravitational versus standard fixed-pressure ventriculoperitoneal shunt valves: a 5 year cohort study in 109 children

Chloe Koh $^{1}$, Jennifer Katy Saunders ${ }^{1}$, Jody O' connor $^{2}$, Kristian Aquilina ${ }^{2}$, Greg James ${ }^{2}$

${ }^{1}$ Barts and the London School of Medicine, Queen Mary University of London, London, UK

${ }^{2}$ Department of Neurosurgery, Great Ormond Street Hospital, London, UK

OBJECTIVE: Ventriculoperitoneal shunts (VPS) are a standard treatment in paediatric hydrocephalus. A number of different valve types are available, with some suggestion that gravitational valves $(\mathrm{GV})$ may confer a benefit compared to traditional fixed-pressure valve (FPV), for example reducing need for revision for malfunction. We studied 5 years outcome for VPS to evaluate time to revision for different valve types.

MATERIAL-METHODS: Retrospective cohort review of all children who underwent primary VPS insertion from 01/01/2013 until 31/12/2014.

RESULTS: 09 children were identified, 63 male and 46 female. Mean age at VPS insertion was 2.5 years (range 1 day-16 years). The most common hydrocephalus aetiology was tumour-related (26), followed by post-haemorrhagic (20), congenital (19), idiopathic/other (16), myelmeningocoele (12), post-infective (4), cranial dysraphism (4), craniosynostosis (3), post-traumatic (2) and vascular (2). 42/109 $(38.5 \%)$ of VPS required revision in the 5 year study period. 38 revisions were for dysfunction (37 underdrainage, 1 overdrainage) and 4 were for infection. The most commonly used 
FPV was Codman Precision (54) and GV was PaediGAV (32). 5 year survival for Precision FPV was $56 \%$ and for PaediGAV was 59\%. Chi squared analysis showed no difference at shunt survival between FPV and GV at 30 days $(\mathrm{P}=0.84), 1$ year $(\mathrm{P}=0.78)$ and 5 years $(\mathrm{P}=0.73)$. Median time to revision for revised shunts were 227.5 days for FPV and 126.5 days for $\mathrm{GV}$.

CONCLUSION: Modern GV designs do not appear to be superior to traditional FPV in terms of shunt survival. There was a trend to earlier failure in GV balanced by lower long-term blockage rate, however this did not reach significance. As per previous studies in the field, there remains an absence of evidence to suggest one type of valve is superior to another in paediatric hydrocephalus.

Keywords: Hydrocephalus, ventriculoperitoneal shunt, shunt valve

\section{FL-039}

\section{Hydrocephalus}

\section{Altered CSF dynamics following surgical intervention} for Chiari malformation in a paediatric population-a 13 year cohort study

Neeraj Kalra, Soumya Mukherjee, John Goodden, Gnanamurthy Sivakumar, Atul Tyagi, Paul Chumas

Department of Paediatric Neurosurgery, Leeds Children's Hospital, Leeds, England

OBJECTIVE: Chiari I malformations are known to affect 1 in 1000 births, and are defined as tonsillar herniation greater than $5 \mathrm{~mm}$ below the foramen magnum. There are reported changes in CSF dynamics associated with surgical decompression for Chiari malformation. We aim to assess our experience with this.

MATERIAL-METHODS: We interrogated our paediatric database for patients who underwent foramen magnum decompression for Chiari malformation in Leeds Children Hospital between February 2006 and February 2019. We subsequently analysed their medical records to assess for any hydrocephalus or change in CSF dynamics either before or after decompression, as well as any CSF diversion undertaken.

RESULTS: 61 paediatric patients (range, $2-15$ years) underwent foramen magnum.

decompression (FMD) for Chiari malformation. Of these, $5(8 \%)$ presented with hydrocephalus, for which they underwent CSF shunting (3 ventriculoperitoneal shunts; 2 ventriculo-atrial shunts), followed by FMD. 50 cases (82\%) underwent FMD with no preceding or post-operative problems with CSF dynamics and who did not require any further procedures. Six cases of Chiari (12\%) did require
CSF diversion following FMD: three cases of post-operative hydrocephalus, for which one underwent ventriculoperitoneal shunting 8 days after FMD, whilst 1 underwent endoscopic third ventriculostomy both more than four years after FMD; one case was complicated by CSF leak and required a lumbar drain for temporary CSF diversion; finally there were 1 cases of persistent headache with small ventricles on $\mathrm{CT}$ and MRI, but with raised pressure on formal intracranial pressure monitoring and lumbar puncture measurements and who underwent ventriculoperitoneal shunting at 12 months following the original FMD surgery with good symptomatic relief.

CONCLUSION: Our data corroborates the wider reports of a complex and not fully understood relationship between Chiari malformation and altered CSF dynamics following surgical decompression.

Keywords: Chiari malformation, intracranial hypotension, cerebrospinal fluid leak, intracranial hypertension, paediatrics

\section{FL-040}

\section{Hydrocephalus \\ Management of Middle Cranial Fossa Arachnoid Cysts: A pilot survey study of members of the Canadian Pedi- atric Neurosurgery Study Group}

Albert $\mathrm{Tu}^{1}$, Pramath Kakodkar ${ }^{8}$, Ziyad Makoshi ${ }^{2}$, Aria Fallah $^{3}$, Alexander Weil ${ }^{4}$, Ash Singhal ${ }^{5}$, George Ibrahim ${ }^{6}$, Aaron Robison ${ }^{7}$, Paul Steinbok ${ }^{5}$

${ }^{1}$ Children's Hospital of Eastern Ontario

${ }^{2}$ University of Ottawa

${ }^{3}$ Mattel Children's Hospital

${ }^{4}$ St Justine Children's Hospital

${ }^{5}$ Children's Hospital of British Columbia

${ }^{6}$ Hospital for Sick Children

${ }^{7}$ Loma Linda University Hospital

${ }^{8}$ Universty of Saskatchewan

OBJECTIVE: Middle cranial fossa arachnoid cysts are a common entity and may be found in association with a variety of presenting symptoms. Management is highly controversial without clear guidelines and anecdotally varies from surgeon to surgeon. We seek to elicit the current management zeitgeist of a selected group of clinicians.

MATERIAL-METHODS: We conducted an online survey distributed to the Canadian Pediatric Neurosurgery Study Group (CPNSG), a cohort of Canadian and Canadian trained pediatric neurosurgeons. 8 clinical scenarios involving Galassi III arachnoid cysts (control, growing, young age, headache, seizure, motor deficit, developmental delay, and cyst hemorrhage) and a variety of patient factors 
were presented as variables that could lead to surgical or conservative management. Interventions were also investigated. Respondent demographics were also characterized to identify potential provider determinants of management. RESULTS: Of the 58 members of the CPNSG surveyed, 21 (36\%) completed the survey. $95 \%$ of respondents were in a consultant practice in North America. Over $80 \%$ of respondents elected non operative management for headache, seizures, young age, developmental delay, hemorrhage into, and a growing cyst. Only 35\% of surgeons intervened for the presence of neurologic deficit. The most common treatment was microsurgical fenestration of the cyst followed by endoscopic fenestration. The presence of papilledema was the most common indication for further intervention in $95 \%$ of respondents.

CONCLUSION: The results of this survey suggest that in this cohort of practitioners, management of Galassi III arachnoid cysts is mostly non surgical, with papilledema being the main indication for further intervention. This survey serves as a pilot for a survey that will be distributed to members of the International Society of Pediatric Neurosurgeons and the final results of which are still pending.

Keywords: arachnoid cyst, survey, papilledema, best management, guidelines

\section{FL-041}

\section{Hydrocephalus \\ Endoscopic treatment for complications related to CSF shunt}

Kenichi Nishiyama

Department of Neurosurgery, Niigata Medical Center, Niigata, Japan

OBJECTIVE: To clarify the management strategy and surgical technique with endoscope for complications related to CSF shunt.

MATERIAL-METHODS: Complications of CSF shunts were roughly classified into four types as follows; Obstruction, over-drainage, infections, Rostral MB syndrome. Endoscopic operations for those four types were reviewed retrospectively. Indications, goals of operation and some special techniques are discussed following the review.

RESULTS: Diversion of CSF pathway with endoscope was a major procedure for functional impairment of CSF shunt, which causes simple-complex hydrocephalus, such as unilateral hydrocephalus or isolated fourth ventricle. This procedure works not only as bypass, but also as buffer for pulsatile pressure in the ventricles. To replace a simple shunt is considered a standard goal. But patients with communicated hydrocephalus including slit-ventricle syndrome were successfully converted into shunt-independent state. ETV was effective for Parkinsonism and mutism i.e. rostral midbrain syndrome following CSF shunt, since repeated attacks of ventricular dilation and relaxation by changing shunt pressure setting makes damages of MB reticular formation. Endoscopic CSF lavage was indicated as an initial treatment for ventriculitis in some cases. In addition, endoscopic choroid plexus cauterization makes time for delay in shunt replacement and was indicated in infectious complications. CONCLUSION: Endoscopic procedures should be considered in the first place for treatment of shunt-related complications. The main objective is re-setting of a simple CSF shunt, however, convert into shunt-independency with resolution of symptoms might be possible in certain cases.

Keywords: neuroendoscope, hydrocephalus, shunt, complication, rostral midbrain syndrome, slit-ventricle syndrome

\section{FL-042}

\section{Hydrocephalus \\ Predicting ETV success with the ETVSS: Does age matter?}

Abbey Boyle $^{1}$, Maisie Ingram ${ }^{1}$, Gnanamurthy Sivajumar ${ }^{2}$, Atul Tyagi ${ }^{2}$, Paul Chumas ${ }^{2}$, Ryan Mathew ${ }^{2}$, John Goodden ${ }^{2}$ ${ }^{1}$ Leeds Medical School, Leeds, UK

${ }^{2}$ Department of Neurosurgery, Leeds General Infirmary, Great George Street, Leeds, LS1 3EX, UK

OBJECTIVE: Assess the validity of the Endoscopic Third Ventriculostomy Success Score (ETVSS) as a short- and long-term clinical predictor of outcomes from endoscopic third ventriculostomy (ETV) at Leeds Children's Hospital (LCH).

MATERIAL-METHODS: 104 patients treated with ETV between January 2006 and March 2019 were retrospectively identified. 14 patients required one repeat ETV, of which 2 required a second repeat ETV: 120 procedures in total. ETVSS was calculated for each patient. The discriminative ability of ETVSS to predict ETV outcome at 30 days, 6-months, 1-, 2- and 5-years was analysed using area under the curve (AUC) analysis. Logistic multivariable regression and chi-squared analyses were used to determine additional prognostic value of age and aetiology.

RESULTS: ETV success rate at 6-months was $57.69 \%$ compared to a mean ETVSS of $71.53 \%$. AUC analysis showed adequate discrimination and therefore predictive value for success at 6 -months by ETVSS (AUC $=0.72$ ). At other follow-up timepoints of 30 days, 1-, 2- and 5-years, inadequate discrimination by ETVSS was shown. Logistic multivariable regression, considering aetiology and age group, demonstrated success rate at 6-months, 1- and 2-years to be 
significantly impacted by patient age. Furthermore, a significant difference between the success rates at 6-months, 1 - and 2-years was seen between patients below and above 24 months of age.

CONCLUSION: The ETVSS shows moderate predictive ability in this cohort at 6-months. However, ETVSS does not appear to hold significant value in predicting success at any other timepoint in our cohort. Age appears to be the key determining factor of ETV success in our cohort from 6 months to 2 years of follow-up. Therefore, we propose an adjustment of the age groups within the ETVSS to reflect the difference in outcomes in patients below and above 24 months of age.

Keywords: paediatric neurosurgery, hydrocephalus, clinical prediction modelling

\section{FL-043}

\section{Hydrocephalus \\ Myelomeningocoele related Hydrocephalus: early vs late treatment}

Taimoor Ali, Muhammad Usman

Neurosurgery department,Lady Reading hospital Peshawar,Pakistan

OBJECTIVE: To compare outcomes of early vs late VP shunt in patients with Myelomeningocele related hydrocephalus.

MATERIAL-METHODS: We studied 42 patients who presented to our unit between September 2017 to December 2019.Patients were divided in two groups.

In the early Group Twenty patients were included with MMC and severe hydrocephalus as diagnosed clinically (showing signs of increased intracranial pressure) and radiologically VP shunt was inserted in same sitting with MMC repair.In the late group twenty two patients were included with MMC and mild hydrocephalus, VP shunt insertion was delayed up to two weeks following MMC repair.

Patients in both groups were followed for twelve weeks for outcomes in-terms of VP shunt infections, CSF leak from MMC repair site, and delayed wound healing of MMC repair site.

RESULTS::The average age was three days with a range of 1 to 5 days and 6 days with a range of 4 to 14 days in early group and late group respectively.Male to female ratio was $1: 1$ in both groups.

In the early group VP shunt infection rate was $25 \%$, CSF leak was $10 \%$,delayed wound healing was $5 \%$ while mortality rate was $10 \%$.
In the late group VP shunt infection rate was $9.09 \%, \mathrm{CSF}$ leak was $40.9 \%$, delayed wound healing was $18.1 \%$ while mortality was $4.54 \%$.

CONCLUSION: Same sitting VP shunt for MMC related hydrocephalus has better outcomes in-terms of CSF leak and wound healing than delayed VP shunt but carries greater risk of VP shunt infection rate.

Keywords: Myelomeningocele,Hydrocephalus, Ventriculop eritoneal shunt, Cerebrospinal fluid leak.

\section{FL-044}

\section{Hydrocephalus}

Paediatric Ventriculo-Atrial Shunts: a 10 year singlecentre experience

Yasir Chowdhury, Katie Herbert, Desiderio Rodrigues, Guirish A Solanki, Anthony R Walsh, William B Lo Department of Neurosurgery, Birmingam Children's Hospital, Birmingham, United Kingdom

OBJECTIVE: The peritoneum is the cavity of choice for cerebrospinal fluid (CSF) shunting. When abdominal contraindications are present then ventriculoatrial (VA) shunts are an alternative route for treatment for hydrocephalus. We present a single-centre experience of indications, outcomes and complications in VA shunts.

MATERIAL-METHODS: A retrospective single-centre case-series analysis of all new VA shunts inserted over a 10 year period.

RESULTS: 13 children ( 5 boys, 8 girls) ranging from ages 46 days to 16 years underwent VA shunting between 2007 and 2017. Indications included: persistent abdominal septated CSF collections (6 children, 46\%); abdominal adhesions (3, 23\%); CSF peritoneal malabsorption $(3,23 \%)$ and recurrent intra-abdominal infection $(1,8 \%)$.

The interval between a child's first VP and VA shunt ranged from 1 day to 15 years. A median $1 \mathrm{VP}$ shunt revision was performed (range 0-6) prior to insertion of a VA shunt. The median age of VA shunt insertion was 7.8 years.

Intraoperatively, open $(5,33 \%)$ and percutaneous $(10$, $67 \%)$ techniques were used. The median follow up from the last VA shunt surgery was 6 years (range: 5 months - 93 months).

There were six short-term $(<30$ days $)$ complications requiring revisions including: cardiac catheter (CC) fracture, valve disconnection, $\mathrm{CC}$ malposition, $\mathrm{CC}$ block, ventricular catheter block and infection. Five long-term ( $>30$ days) complications requiring revisions included $2 \mathrm{CC}$ blockages, early removal (suspected infection), $\mathrm{CC}$ fracture and $\mathrm{CC}$ 
malposition. Two children with VA shunts required conversion back to VP shunts. After further abdominal complications both were changed back to VA shunt.

CONCLUSION: Our case-series did not identify any severe cardiopulmonary or shunt nephritis morbidity that is historically associated with the procedure. The majority of complications were surgery or hardware-related.

Intraoperative techniques varied widely amongst this cohort of patients. The frequency of VA shunts will remain low, developing a standardised procedural technique may lead to lower complication rates.

Keywords: Hydrocephalus Ventriculoatrial shunt CSF

\section{FL-045}

\section{Hydrocephalus}

Standardized protocol for perioperative managements of CSF shunting in hydrocephalic infants decreases shunt infection and shunt malfunction: a retrospective singlecenter study

Naoki Kagawa $^{1}$, Yasuyoshi Chiba ${ }^{2}$, Ryuichi Hirayama ${ }^{1}$, Hitomi Arahori ${ }^{3}$, Yasuji Kitabatake ${ }^{3}$, Tomoyuki Maruo ${ }^{4}$, Chisato Yokota $^{5}$, Tomoyoshi Nakagawa ${ }^{1}$, Masahiro Nonaka ${ }^{6}$, Kazuko Wada ${ }^{7}$, Haruhiko Kihsima ${ }^{1}$.

${ }^{1}$ Department of Neurosurgery, Osaka University Graduate School of Medicine, Osaka, Japan

${ }^{2}$ Department of Neurosurgery, Osaka Women's and Children's Hospital, Osaka, Japan

${ }^{3}$ Department of Pediatrics, Osaka University Graduate School of Medicine, Osaka, Japan

${ }^{4}$ Department of Neurosurgery, Otemae Hospital, Osaka, Japan

${ }^{5}$ Department of Neurosurgery, Suita Municipal Hospital, Osaka, Japan

${ }^{6}$ Department of Pediatric Neurosurgery, Kansai Medical University Hirakata Hospital, Osaka, Japan

${ }^{7}$ Department of Pediatrics, Osaka Women's and Children's Hospital, Osaka, Japan

OBJECTIVE: Shunt infection and shunt malfunction are common complications for CSF shunting in hydrocephalic infants. Prevention of these complications in shunt surgery is important for improvement of clinical outcome and reduction of hospital cost. In Japan, many young neurosurgeons and other medical staffs participate in shunt surgeries and perioperative management at University hospitals like our institution. In an effort to improve the clinical outcome of ventriculoperitoneal shunting, a standardized protocol was developed with the cooperation of some expert pediatric neurosurgeons and pediatricians. We analyzed clinical parameters associated with shunt infection and shunt malfunction and estimated the effect of the protocol associated with perioperative management.

MATERIAL-METHODS: Standardized protocol containing preoperative and postoperative management was introduced at shunt surgeries in in children from 2007 at Osaka University Medical Hospital. 235 ventriculoperitoneal shunting procedures for 128 children were performed from 1994 to 2019. We compared some clinical outcome and parameters before (group A) and after (group B) standardized protocol. We analyzed infection rate, shunt survival time, CRP value, duration of antibiotics administration, other clinical parameters between group A and group B.

RESULTS: Total infection rate of all shunting procedures was $10.2 \%$. Infection rate was $9.5 \%$ in infants under 12 months of age. Infection rate for infants significantly decreased from $18.4 \%$ (group A) prior to the protocol to $2.0 \%$ (group $B$ ) while using the protocol $(\mathrm{p}<0.01)$. In group $\mathrm{B}$, both administration period of antibiotics and highest value of CRP remarkably improved due to introduction of the protocol. Shunt survival time was prolonged by the protocol $(\mathrm{p}=0.027)$.

CONCLUSION: Standardized protocol for perioperative practice can improve shunt infection rate and shunt survival time and reduce hospital cost, should be used in other educational institution. Further multicenter study is needed to verify effectiveness of this protocol.

Keywords: CSF shunting, infantile hydrocephalus, perioperative management, standardized protocol, infection rate, shunt survival time

\section{FL-046}

\section{Hydrocephalus \\ Aqueductal Stenosis in Children}

Anna Prajsnar Borak, Joachim Oertel

Department of Neurosurgery, University Hospital of Saarland, Homburg, Germany

OBJECTIVE: Endoscopy for obstructive hydrocephalus has become well-established treatment option. However, endoscopic management of aqueductal stenosis (AS) -related hydrocephalus in children, due to its peculiar characteristics and multifold etiology is associated with varied success rate. We report on our experiences for CSF restoration due to AS. MATERIAL-METHODS: Between December 2010 and April 2020, 41 endoscopic procedures were performed in 27 children with AS (13 males, 14 females, mean age 7,3 years, range 1 month to 18 years). Idiopathic AS (10 cases), isolated fourth ventricle after shunting ( 5 cases), posthemorrhagic AS (4 cases), tumour (4 cases), pineal cyst (1 case), post-inflammatory AS (1 case), AS associated with 
septo-optic dysplasia (1 case) and vascular malformation with complete occlusion of aqueduct (1 case), caused the AS -related hydrocephalus. In 15 patients, endoscopic third ventriculostomy (ETV) was the first treatment option and in 6 children, second procedure of choice. Simultaneously with ETV, endoscopic tumour biopsy (3 cases), pineal cyst fenestration ( 1 case) and tumour resection (1 case) were performed. 9 endoscopic transaqueductal stents were placed in 6 children.

RESULTS: Success rate varied in regard to underlying pathology. Postoperatively, 19 children (70\%) with hydrocephalus-related, clinical symptoms, improved or resolved completely, and 8 patients (30\%) presented with stable course. There were no intraoperative complications, and no endoscopy-related morbidity. Two patients with postoperative wound healing and one child with postoperative ventriculitis, underwent revision surgery. Shunt dependency was verified in 14 children (51\%). The mean follow-up period, ranged from 3 to 288 months, mean; 71,5 months.

CONCLUSION: Underlying mechanism and cause of aqueductal stenosis in children plays an imperative role in decision making and selection of the primary treatment strategy, determining success rate. Endoscopic transaqueductal stenting is a valuable alternative for IFV. ETV is feasible first line treatment option for tumour related-, and idiopathic AS.

Keywords: Hydrocephalus, Endoscopy, Transaqueductal Stent Placement, Aqueductal Stenosis

\section{FL-047}

\section{Hydrocephalus \\ Fourth ventricular shunts in the paediatric patients: sur- vival analysis}

Claudia L Craven, Adikarige Silva, Kristian Aquilina Department of Paediatric Neurosurgery, Great Ormond Street Hospital for Children NHS Foundation Trust, London, United Kingdom

OBJECTIVE: To determine the safety profile and longevity of fourth ventricular shunts in paediatric patients.

MATERIAL-METHODS: A single centre retrospective cohort of paediatric patients requiring a fourth ventricular shunt, between 2007-2020. Inclusion criteria: Any patient under the age of 18 years, who underwent any fourth ventricular catheter insertion. Baseline demographic data was obtained from a prospectively maintained operative database. Outcome measures included shunt complication rates and survival, analysed using a Kaplan-Meier curve.

RESULTS: Demographics: A total of 11 fourth ventricular shunt operations were performed in ten patients (3 M: $7 \mathrm{~F})$ aged $3 y \mathrm{~m} \pm 4 \mathrm{y} 5 \mathrm{~m}(\mathrm{mean} \pm \mathrm{SD})$, between 2007 and
2020. Indications for fourth ventricular catheterisation included trapped fourth ventricle (TFV) and Dandy-Walker syndrome. Two patients had an associated syrinx which improved post shunting.

Shunt constructs: Of the 11 shunt procedures, eight were additions of a fourth ventricular catheter to an existing supratentorial shunt construct, two were single fourth ventricular catheters, and one was a revision/replacement of the fourth ventricular catheter. Four were inserted with assistance of neuronavigation.

Complications: There was a single case of proximal obstruction, occurring 5 months post catheter insertion. The proximal fourth ventricular catheter was subsequently replaced. There were no other reported shunt related complications in this group.

Survival: Median follow-up was 64.2 months (range 11 days -158 months). Shunt failure in this given follow-up period is a rare event, therefore median survival is undefined (greater than 50\% at the last time point of 158 months) and minimum median survival is 13 years.

CONCLUSION: Fourth ventricular shunt placement appears to be a safe and robust treatment option for patients requiring infra-tentorial CSF diversion. Furthermore, it may be less invasive than alternative surgical techniques, such aqueductoplasty or open posterior fossa surgery.

Keywords: Fourth ventricular shunt, Hydrocephalus, Dandy-Walker, Encysted fourth ventricle

\section{FL-048}

\section{Hydrocephalus}

The impact of age on the rate of ventriculoperitoneal shunt failure in patients with hydrocephalus

Alexandra Jayne Nelson $^{1}$, Geraint Sunderland ${ }^{2}$, Elizabeth Conroy $^{3}$, Michael Jenkinson ${ }^{4}$, Conor Mallucci ${ }^{2}$

${ }^{1}$ University of Liverpool, Liverpool, United Kingdom

${ }^{2}$ Department of Paediatric Neurosurgery, Alder Hey Children's Hospital, Liverpool, United Kingdom

${ }^{3}$ Clinical Trials Research Centre, University of Liverpool, Liverpool, United Kingdom

${ }^{4}$ Department of Neurosurgery, Walton Centre National Health Service Foundation Trust, Liverpool, United Kingdom

OBJECTIVE: To identify the impact of age on ventriculoperitoneal shunt (VPS) failure rate after initial shunt placement for patients with hydrocephalus.

MATERIAL-METHODS: Analysis of derived data sets from a multicentre, single-blind randomised controlled trial (BASICS). BASICS included 1605 patients with hydrocephalus of any aetiology and age undergoing de novo VPS 
insertion. Details on patient demographics, aetiology of hydrocephalus, valve type and shunt revision operations were collected from the data sets.

RESULTS: Of the 1605 patients; 817 (51\%) were male, 788 (49\%) were female and the median age of VPS insertion was 42.5 years old (IQR 0.8-69.6). Patients were followed up between 6-24 months (median 22 months (IQR 10-24)). There were 398 shunt revisions (25\%) for all causes during the follow up period of the trial. The overall infection rate was approximately $5 \%(\mathrm{n}=78)$ and the overall clean revision rate was approximately $19 \%(\mathrm{n}=304)$. Neonatal patients ( $<1$ month old) had the highest rate of shunt failure for all causes at $45 \%$ and elderly patients ( $>65$ years old) had the lowest rate of shunt failure for all causes at $12 \%$ (RR 3.673, CI 2.726-4.949). Rate of shunt failure in 1 month to 1 -year-olds, 1 to 5 -year-olds, 5 to 16 -year-olds, 16 to 65 -year-olds was $37 \%, 33 \%, 31 \%$ and $23 \%$ respectively. Kaplan Meier curves indicated a significant difference in the rate of shunt failure between all six age categories which was proportional over time ( $p<0.01$, df 5$)$. Age is significantly associated with the rate of shunt failure with each advancing year decreasing the risk of shunt revision by $2 \%$ and each advancing decade decreasing the risk of revision by $18 \%$ (HR 0.98, CI 0.979-0.986, $\mathrm{p}<0.01$ ).

CONCLUSION: Age is independently associated with the rate of shunt failure in paediatric patients with VPS, in particular neonates and those born preterm are at highest risk.

Keywords: Hydrocephalus, ventriculoperitoneal shunt, shunt failure

\section{FL-049}

\section{Hydrocephalus}

CSF Diversion for Post-Hemorrhagic Hydrocephalus: Predictive Factors for Definitive CSF Diversion in a Reservoir Wean Trial

Jackson Pearce $^{1}$, Chistopher Litts ${ }^{1}$, Thomas Larrew ${ }^{2}$, Mohammed Alshareef ${ }^{2}$, Libby Infinger ${ }^{2}$, Tyler Vasas ${ }^{1}$, Paul Nietert $^{3}$, Ramin Eskandari ${ }^{2}$

${ }^{1}$ College of Medicine, Medical University of South Carolina, Charleston, USA

${ }^{2}$ Department of Neurosurgery, Medical University of South Carolina, Charleston, USA

${ }^{3}$ Department of Public Health Sciences, Medical University of South Carolina, USA

BACKGROUND AND AIM:Post-hemorrhagic hydrocephalus (PHH) continues to account for significant morbidity and.

mortality in preterm infants, and protocols for temporizing treatment and the need for definitive cerebrospinal fluid
(CSF) diversion vary dramatically. We report findings from a modified protocol of early ventricular reservoir placement with prolonged weaning via ventricular taps and the implications for permanent CSF diversion.

METHODS:An institutional IRB-approved weaning trial is currently active in which preterm infants with PHH follow a weight-based ventricular reservoir tapping protocol until 38 weeks gestational age (GA), and then undergo an attempted wean with staggered reservoir taps following three stable head ultrasounds. 27 patients from 2017-2021 with ventricular reservoirs placed for PHH attempted a wean trial. Demographics, ventricle size, and outcomes were analyzed. Biventricular diameter and third ventricle diameter at the foramen of Monro (BVD-FoM, 3 V-FoM) were used for ventricular measurements.

RESULTS: 39 patients were included with 12 in the weightbased tapping group and 27 in the imaging-based tapping group. All patients in the weight based-tapping group had permanent CSF diversion at 6-month follow up, while $31 \%$ (12 of 27) in the imaging-based tapping group were successfully weaned without permanent CSF diversion at last follow up. Weaned patients were older at birth than patients requiring, $p=0.01$. Weaned patients were older at reservoir placement compared to VPS patients, $p=0.011$. Weaned patients were heavier at birth compared to those requiring VPS, $\mathrm{p}=0.011$, and also heavier at reservoir placement than VPS patients, $\mathrm{p}=0.04$.

CONCLUSIONS:Non-modifiable factors such as age and birthweight are predictive of successful weaning. Our ongoing weaning protocol demonstrates promising results regarding trends in a modifiable factor, BVD-FoM at 38 weeks GA, which will require increased patient enrollment to establish statistical significance.

Keywords: hydrocephalus, cerebrospinal fluid diversion, post-hemorrhagic hydrocephalus, ventriculoperitoneal shunts

\section{FL-050}

\section{Hydrocephalus}

Post-haemorrhagic hydrocephalus is associated with poorer surgical and neurodevelopmental outcomes than other aetiologies of infantile hydrocephalus

Malak Mohamed ${ }^{1}$, Saniya Mediratta ${ }^{2}$, Aswin Chari ${ }^{3}$, Gregory James ${ }^{3}$, William Dawes ${ }^{4}$, Kristian Aquilina ${ }^{3}$

${ }^{1}$ Great Ormond Street Institute of Child Health, University College London, London, United Kingdom; Leeds School of Medicine, University of Leeds, Leeds, United Kingdom ${ }^{2}$ Division of Neurosurgery, Department of Clinical Neurosciences, Addenbrooke's Hospital \& University of Cambridge, Cambridge, United Kingdom 
${ }^{3}$ Department of Neurosurgery, Great Ormond Street Hospital, London, United Kingdom

${ }^{4}$ Department of Neurosurgery, Alder Hey Children's Hospital, Liverpool, United Kingdom

BACKGROUND AND AIM:This retrospective cohort study aimed to investigate the surgical and neurodevelopmental sequelae of infantile hydrocephalus, and to evaluate whether outcomes are disproportionately poorer in posthaemorrhagic hydrocephalus ( $\mathrm{PHH}$ ) compared to other hydrocephalus aetiologies.

METHODS:All infants with hydrocephalus, who had ventriculoperitoneal shunts inserted at Great Ormond Street Hospital between 2008 and 2018, were evaluated. Demographic, surgical, and neurodevelopmental data were extracted from electronic health records. Shunt survival, neurodevelopmental outcome (NDO), cerebral palsy, epilepsy, speech delay, education, behavioural disorders, endocrine dysfunction, and mortality were analysed by aetiology. RESULTS: 323 infants with median gestational age 37.0 (23.29-42.14) weeks and birthweight $2640 \mathrm{~g}$ (525-4684 g) were evaluated. The most common aetiology was PHH (31.9\%), which was associated with a significantly higher five-year shunt revision rate (62.7\%), revision beyond a year (40.7\%), and median number of revisions (1) than congenital or 'other' hydrocephalus ( $\mathrm{p} \leq 0.01)$. Cox regression demonstrated poorest shunt survival in $\mathrm{PHH}$, related to gestational age at birth $(\mathrm{p}=0.01)$ and corrected age at shunt insertion $(\mathrm{p}=0.01)$.

PHH had the highest severe disability rate, increasing with age to $65.0 \%$ at ten years, compared to genetic (45.5\%), 'other' (22.2\%), congenital hydrocephalus (18.2\%) and neural tube defects $(0 \%)$; multivariate regression demonstrated significant differences in NDO between aetiologies at one $(\mathrm{p}=0.01)$, two $(\mathrm{p}<0.01)$ and five years $(\mathrm{p}=0.03)$. PHH also had the highest cerebral palsy rate $(62.6 \%, \mathrm{p}<0.01)$. While epilepsy $(43.2 \%, \mathrm{p}<0.01)$, speech delay $(82.8 \%$, $\mathrm{p}<0.01)$ and special education rates $(50.0 \%, \mathrm{p} \leq 0.01)$ were significantly higher in PHH than in some aetiologies, odds of behavioural disorders did not differ between groups $(p=0.23)$. Only genetic hydrocephalus had significantly higher odds of endocrine dysfunction $(p=0.01)$ and mortality $(\mathrm{p}=0.04)$ than PHH.

CONCLUSIONS:PHH is associated with poorer surgical and NDO than other hydrocephalus aetiologies, excluding genetic hydrocephalus. Research is urgently required into measures of reducing neurodisability following PHH. Long-term follow-up is essential to optimise support and outcomes.

Keywords: hydrocephalus, intraventricular haemorrhage, neonates, ventriculoperitoneal shunts, neurodevelopmental outcome
FL-051

\section{Hydrocephalus \\ Ventriculoperitoneal shunt drainage increases with grav- ity and cerebrospinal fluid pressure pulsations: benchtop model}

Joyce Koueik $^{1}$, Bermans J Iskandar ${ }^{1}$, Zhe Yang ${ }^{2}$, Kraemer Mark $^{1}$, Armstrong Stephanie ${ }^{1}$, Aimee Broman ${ }^{4}$, Victor Wakim $^{1}$, Joshua Medow ${ }^{5}$, Christopher Luzzio ${ }^{6}$, David $\mathrm{Hsu}^{3}$ ${ }^{1}$ Departments of Neurological Surgery

${ }^{2}$ Electrical Engineering

${ }^{3}$ Neurology

${ }^{4}$ Biostatistics and Medical Informatic

${ }^{5}$ Biomedical Engineering5

${ }^{6}$ Mechanical Engineerin

BACKGROUND AND AIM:There have been few improvements in modern shunt technology since John Holter introduced the silicon valve, with overdrainage remaining a major source of complications. The present study aims to better understand why valves are afflicted by supra-normal CSF flow rates under physiologic conditions. We present in-vitro benchtop analyses of fluid flow through a differential pressure valve under simulated physiological conditions.

METHODS:The PV benchtop valve testing platform that comprises a rigid pseudo-ventricle, compliance chamber, pulsation generator and pressure sensors was used to measure flow rates through a differential pressure shunt valve under the following simulated physiological conditions: orientation (horizontal and vertical), compliance (low, medium and high), and pulsation generator force (low, medium and high).

RESULTS: Our data show that pulse pressures are faithfully transmitted from the ventricle into the shunt (ventricular) catheter; that lower compliance and higher pulse generator forces lead to higher pulse pressures in the pseudo-ventricle; and that both gravity and higher pulse pressure lead to higher flow rates. The presence of a valve mitigates but does not eliminate these higher flow rates.

CONCLUSIONS:It's well understood that CSF shunt valves are prone to overdrainage related to gravitational forces in the vertical position, which has motivated the development of gravitational valves and antisiphon devices. This study indicates that overdrainage is not limited to the vertical position, but that in addition, pulse pressures that simulate physiological and pathological CSF pressure pulsations increase outflow in both the horizontal and vertical positions, and are dependent on (brain) compliance. A deeper understanding of the physiological parameters that affect intracranial pressure and CSF flow through shunt systems is necessary prerequisite to the design and development of novel valves. 
Keywords:: Hydrocephalus, Cerebrospinal fluid (CSF) shunts, Shunt malfunction, CSF Overdrainage

\section{FL-052}

\section{Infection}

Predictors and surgical outcome of spontaneous subdural empyema in children: An institutional experience

\author{
Subhas Konar ${ }^{1}$, Dhaval Shukla ${ }^{2}$ \\ ${ }^{1}$ Subhas Konar, Dept. of Neurosurgery, NIMHANS, \\ Bangalore \\ ${ }^{2}$ Dhaval Shukla,Dept. of Neurosurgery, NIMHANS, \\ Bangalore
}

OBJECTIVE: The aim of the study was to report the aetiology, clinical features, microbiology, surgical outcome and other various predictors related with the outcome of spontaneous subdural empyema.

MATERIAL-METHODS: This is a retrospective study conducted in a tertiary hospital for 12 years. Children, up to 18 years with a diagnosis of subdural empyema (spontaneous) with infective aetiology were included in the present cohort. Post traumatic, post-surgery and tubercular aetiology of subdural empyema were excluded from the study. Glasgow outcome scale was used for outcome assessment at the end of three months. For analysis purpose, the demographic data, clinical features, radiological data, microbiology, type of surgery, complication data were categorized, and univariate and regression analysis were performed to identify the factors associated with outcome.

RESULTS: Ninety-eight (98) children were included in the study and the mean age was 10.8 years. Otogenic origin $(34.6 \%)$ was the maximum followed by meningitis (14.2\%) 0.76 children presented with GCS more than 8 and the supratentorial location was the commonest location.37\% children had sterile culture and Beta-haemolytic Streptococcus $(10 \%)$ is the commonest organism.73.5\% children underwent craniotomy or craniectomy and rest burr-hole evacuation. Cortical venous thrombosis $(10.2 \%)$ is the commonest complication in our cohort. The other complications were infraction (6.1\%), new-onset seizure (4.08\%), bone flap osteomyelitis (4.08\%). Thirteen cases had a recurrence of pus collection and common in craniotomy (11 cases) in compare to the burr-hole group ( 2 cases). We have found that age less than 10 years (OR:0.21, $\mathrm{P}=0.03), \mathrm{GCS}<8(\mathrm{OR}: 0.11$, $\mathrm{P}=0.002)$, cortical venous thrombosis (OR:15.17, $\mathrm{P}=0.001)$ and presence of infraction $(\mathrm{OR}: 7, \mathrm{P}=0.05)$ were strongly associated with unfavourable outcome. In regression analysis, only GCS less than $8(\mathrm{P}=0.002)$ and presence of infraction $(\mathrm{P}=0.01)$ had a significant impact on the unfavourable outcome.
CONCLUSION: Prompt diagnosis and immediate intervention is the goal of management of subdural empyema especially in children.

Keywords: Children,Subdural,Empyema,Outcome,Predic tor

\section{FL-053}

\section{Infection}

The Effect of External Ventricular Drain Tunneling Length on CSF Infection rate in Pediatric Patients: A Randomized, Double-blind, Three-arm Controlled Trial

Zohreh Habibi, Esmaeil Mohammadi, Sina Azadnajafabad, Sara Hanaei, Keyvan Tayebi Meybodi, Farideh Nejat Department of pediatric neurosurgery, Children's Medical Center Hospital, Tehran University of Medical Sciences, Tehran, Iran

BACKGROUND AND AIM:The role of tunneling of external ventricular drain (EVD) more than standard $5 \mathrm{~cm}$ for controlling device-related infections remains controversial. METHODS:This is a randomized, double-blind, three-arm controlled trial done in Children's Medical Center in Tehran. Patients ( $<18$ years-old) with temporary hydrocephalus requiring EVD and no evidence of CSF infection or prior EVD insertion were enrolled. Patients were randomly assigned (1:1:1) into arms A:5 [standard], B:10, or C:15-cm EVD tunnel lengths. The investigators, parents, and analyst were masked. The surgeon was informed of the length just before operation by monitoring board. Patients were followed until EVD's fate was established. Infection rate and other complications related to EVD were assessed. This study is registered with IRCT.ir [IRCT20160430027680N2]. RESULTS: 105 patients were enrolled in three random groups $(\mathrm{A}=36, \mathrm{~B}=35$, and $\mathrm{C}=34)$. $\mathrm{EVD}$ was removed due to no further need in most cases $(67.6 \%)$, followed by converting to new EVD or ventriculoperitoneal shunt (15.2\%), infection (11.4\%), and spontaneous discharge without further CSF diversion requirement (5.7\%). No statistical difference was found in infection rate $(\mathrm{P}$-value $=0.47)$ or EVD duration $(\mathrm{P}$-value $=0.81)$ between three groups. No group reached the efficacy point sooner than standard group (B: hazard ratio 1.21 [95\% confidence interval: 0.75-1.94], $\mathrm{P}$-value $=0.429 ; \mathrm{C}: 1.03$ [0.64-1.65], P-value $=0.91$ ).

CONCLUSIONS:EVD tunnels of $5 \mathrm{~cm}$ and longer did not show difference in the infection rate in pediatric patients. Indeed, tunneling lengths of $5 \mathrm{~cm}$ and greater seem to be equally effective in preventing EVD infection. [Accepted to be published in Journal of Neurosurgery Pediatrics, https:// doi.org/10.3171/2020.9.PEDS20748]. 
Keywords: Hydrocephalus, External Ventricular Drain, Tunneling Length, Infection, Pediatric, Randomized Controlled Trial

\section{FL-054}

\section{Infection}

Implementation of a Shunt Insertion Protocol and its Effect on Institutional Shunt Infection Rates

Miriam Claire Ortua Dy ${ }^{1}$, Juan Silvestre Grecia Pascual ${ }^{1}$, Ronnie Enriquez Baticulon ${ }^{2}$, Kathleen Joy Ong Lopez Khu ${ }^{1}$ ${ }^{1}$ Division of Neurosurgery, Department of Neurosciences, University of the Philippines - Philippine General Hospital ${ }^{2}$ Department of Anatomy, College of Medicine, University of the Philippines Manila

BACKGROUND AND AIM:The shunt protocol developed by the Hydrocephalus Clinical Research Network (HCRN) significantly reduced institutional ventriculoperitoneal shunt infection rates. The present study determined the effect of implementing a standardized shunt infection prevention protocol on cerebrospinal fluid (CSF) shunt infection rates at the Philippine General Hospital (PGH).

METHODS:The PGH shunt protocol, closely adapted from the 2007 HCRN shunt protocol was prospectively implemented in all CSF shunting procedures at PGH from July 1, 2019 to March 31, 2020. All patients shunted from July 1, 2018 to June 30, 2019 served as the control cohort. Primary outcome was shunt infection.

RESULTS: A total of 194 procedures were performed after protocol implementation and a control cohort consisted of 269 procedures. Sex (male $52.78 \%$ vs $49.48 \%$ ) and age (pediatric age in $74.34 \%$ vs $77.83 \%$ ) distribution among control and post protocol cohorts were identical. Shunt infection rates decreased after implementation of the PGH shunt protocol $(11.89 \%$ vs $7.73 \%, p=0.094)$. Perfect compliance to the protocol was observed in $26.28 \%$ of procedures, while 10 of the 11 steps were adhered to in $38.65 \%$. No protocol factors were associated with shunt infection on univariate analysis.

CONCLUSIONS:The implementation of a standardized shunt protocol showed a trend toward reduction in shunt infection rates at the Philippine General Hospital. The study has established a standardized peri-operative and intra-operative care for patients undergoing shunting procedures in the institution. Future studies may be geared toward assessment of new interventions or increasing adherence to a shunt protocol.

Keywords: Hydrocephalus, shunt infection, standardized protocol

\section{FL-055}

Innovation and technology

Technological innovations in the management of spinal cord tumors in children

Luca Massimi, Paolo Frassanito, Federico Bianchi, Gianpiero Tamburrini

Neurochirurgia Pediatrica, Fondazione Policlinico Universitario A. Gemelli IRCCS, Roma, Italy

OBJECTIVE: Some tools to improve the management of pediatric spinal cord tumors (pSCT) are now a standard option (e. g. intraoperative neurophysiological monitoring, INM), while others have been recently introduced (e. g. piezosurgery, exoscope). Herein, we present our experience with piezosurgery and exoscope.

MATERIAL-METHODS: All children with pSCT consecutively admitted to our Institution between 2014 (when piezosurgery was acquired) and 2019 were considered (mean follow-up: 2.9 years). The comparison between piezorurgery (group A) and traditional osteotomes (group B) was based on the following variables: surgical times (laminotomy alone), dural tears, estimated blood loss (EBL) and transfusion rate, spinal sequelae (cifosis, scoliosis). The exoscope has been used in the last 5 cases; therefore, it is presented with iconographic purposes.

RESULTS: 23 children (mean age: 7,4 years, M/F ratio: 0,8) underwent surgery under INM and microscopic magnification except for the last 5 cases (INM and exoscope). Group A consists of 12 children where laminotomy was realized by piezosurgery; group B is composed by 11 children treated by traditional osteotome. With the limit of the small number of cases and the relatively short follow-up, no signifcant differences between the two groups were observed about EBL, transfusion rate and spinal sequelae. Two dural tears occurred in group B. The operating times were longer in group A, although significantly reduced by the introduction of piezosurgery plus. Exoscope provided a magnification of the surgical details superior than microscope; a learning curve is required, though neurosurgeons confident with neuroendoscopy are favored. These results are discussed also in comparison to the historical series of spinal cord tumors. CONCLUSION: The techincal and anatomical skills of the neurosurgeon together with the use of INM remain the main factors affecting the outcome of pSCT. However, the introduction of new tools, like piezosurgery and exoscope, can promote the reduction of postoperative complications.

Keywords: spinal cord tumors, piezosurgery, exoscope

\section{FL-056}


Innovation and technology

State of the art in neurosurgery: navigation-assisted neuroendoscopy. Our Experience

Dan Aurel Nica, Ramona Manuela Savu, Maria Alexandra Andrei, Horatiu Alexandru Moisa

Department of pediatric neurosurgery, "Grigore Alexandrescu" Emergency Children's Hospital, Bucharest, Romania

OBJECTIVE: Neuronavigation-assisted neuroendoscopy (NAN) is a relatively new technique which combines the use of an endoscope with that of real-time neuronavigation. The result of this combination renders endoscopy to be easier and safer to perform, offering neurosurgeons the chance to tackle complex pathologies otherwise extremely difficult to treat. In this retrospective study the authors review their experience using neuronavigation-assisted endoscopy for the treatment of complex neurosurgical cases in children.

MATERIAL-METHODS: In the 4 years since the inauguration of our clinic, we performed 350 surgical interventions, out of which 32 were endoscopies. We approached patients with rare tumors (such as dumbbell trigeminal schwannoma) multilocular hydrocephalus, brainstem tumors and basal ganglia tumors. Our study reviews our experience with classic endoscopy and NAN and performs a comparisson between the two subsequently highlighting indications as well the pros and cons of using both techniques.

RESULTS: In our series of cases there was no techniquerelated fatality. All cases in which NAN was used were carefully selected and the purpose of surgery was achieved. Even though oprational success was achieved, there were some cases - including malignancies or refractory multilocular hydrocephalus where treatment failed.

CONCLUSION: Navigated neuroendoscopy is a safe technique which can be used in treating difficult neurosurgical cases in pediatric neurosurgery. Real time location feed-back coupled with visual double-checking offers a large degree of comfort to the operating surgeon. Future perspectives for endoscopy are bright as the progress of science and robotics allows for further miniaturization, better optics, better control and better instruments.

Keywords: endoscopy, navigation, rare conditions, techniques, experience

\section{FL-057}

\section{Innovation and technology 3D and virtual reality applications in neurosurgical oncology}

Andrea Carai ${ }^{1}$, Nicola Onorini ${ }^{1}$, Angela Mastronuzzi ${ }^{2}$, Giovanna Stefania Colafati ${ }^{3}$, Alessia Carboni ${ }^{3}$, Eleonora Piccirillo $^{3}$, Giada Del Baldo ${ }^{2}$, Carlotta Ginevra Nucci ${ }^{1}$, Alessandro De Benedictis ${ }^{1}$, Carlo Efisio Marras ${ }^{1}$

${ }^{1}$ Neurosurgery Unit, Bambino Gesù Children's Hospital, Rome, Italy

${ }^{2}$ Neuro-Oncology Unit, Bambino Gesù Children's Hospital, Rome, Italy

${ }^{3}$ Imaging Department, Bambino Gesù Children's Hospital, Rome, Italy

${ }^{4}$ Department of Radiology, University of Chieti, Chieti, Italy

OBJECTIVE: Tridimensional (3D) rendering of volumetric neuroimaging sequences is becoming increasingly popular in neurosurgical practice. The recent availability of virtual reality (VR) applications is a very promising tool to assist in planning and execution of complex surgical procedures. MATERIAL-METHODS: We describe the use of the Surgical Theatre, a commercially available 3D VR workstation, in a series of 80 consecutive cases.

3D VR information was used in different settings: planning of the procedure, sharing of information within the team and with families at the time of informed consent, intraoperatively, post-operatively as a debriefing tool.

Benefits and limitations of the system for a well established pediatric neurosurgical practice were recorded.

RESULTS: The Surgical Theatre allowed a very effective reconstruction of virtual anatomical models in all cases. Quality of the rendering is highly influenced by image quality for which a strong cooperation with neuro-radiology is mandatory.

3D VR was extremely effective during MDT discussions, influencing treatment strategy, especially in selected cases. Use during informed consent was always beneficial, allowing a faster and more effective communication. $15 \%$ families refused to use VR, fearing it would have been a traumatic experience.

Use during and after surgery was mostly beneficial in deep seated supratentorial lesions, intra-axial brainstem and cerebellar tumors and for lesions with significant vascular relationships. Integration with the navigation system allowed a precisely tailored approach in all cases.

CONCLUSION: The Surgical Theatre is a very promising in the setting of pediatric neurosurgical oncology. Our preliminary experience suggests it significantly contributes to surgical quality, even in the hand of an experienced pediatric neurosurgical team.

More data will be necessary to improve its use as a communication tool with families.

Keywords: Virtual Reality, 3D, neurosurgical oncology, brain tumor 


\section{FL-058}

\section{Innovation and technology}

'Dynamic' intraoperative ultrasound (dIOUS): How pressure irrigation of the surgical cavity may enhance IOUS diagnostic accuracy

Gianpiero Tamburrini ${ }^{1}$, Paolo Frassanito ${ }^{2}$, Luca Massimi ${ }^{1}$, Lazaro De Lima ${ }^{2}$, Federico Bianchi ${ }^{2}$

${ }^{1}$ Catholic University Medical School, Rome, Italy

${ }^{2}$ Fondazione Policlinico Gemelli, IRCCS, Rome, Italy

OBJECTIVE: To evaluate the role of dynamic intraoperative US (dIOUS) as a technique to improve the reliability of intraoperative US after intracranial lesions removal.

MATERIAL-METHODS: Ten consecutive patients operated for an intracranial lesion in the last 6 months at our Institution were included in the present study. A 'dynamic' IOUS (dIOUS) assessment of the surgical cavity was performed in all cases: namely, at the end of the microsurgical excision of the "tumor", the microconvex probe was used to perform an ultrasound examination of the surgical field before and after a gentle pressure injection of Ringer lactate, this way inflating the residual cavity and stretching its walls.

RESULTS: The dIOUS assessment improved the interpretation of the IOUS images in all cases. It specifically allowed to distinguish between true and false hyperechoic nodular images, which were present at the end of surgery and might have suggested a "tumor" residual.

Concordance of dIOUS with postoperative MR was confirmed after surgery in all the patients.

CONCLUSION: Surgical debris, air as well as the collapse of the surgical cavity may hamper the correct interpretation of IOUS findings after microsurgical "tumor" excision. To overcome this limit, the acquisition of dIOUS scans represented in this preliminary experience a useful and reproducible technical adjunct.

Keywords: intraoperative ultrasound, tumor removal

\section{FL-059}

\section{Innovation and technology}

Deep Learning-Based Single-Cell RNA Sequencing Differentiation Identifies Simple and Complex Transcriptional Networks for Subpopulation Classification

\section{Eric Prince, Todd Hankinson}

Department of Neurosurgery, University of Colorado Anschutz Medical Campus, Aurora, USA
OBJECTIVE: Genomic assays capable of cellular resolution (i.e. scRNA-seq) are becoming ubiquitous in biomedical research. Machine learning, and the subtype known as Deep Learning, have broad application within scRNA-seq analytics. However, methods to facilitate the classification of cell populations are lacking. We present the novel computational framework HD Spot, which generates interpretable and robust Deep Learning classifiers that enable unbiased interrogation of linear and non-linear genomic signatures. MATERIAL-METHODS: HD Spot is written in python and relies on Google's TensorFlow2 deep learning framework. Four test datasets of immune cells were obtained from the publicly available Seurat repository, generated using the 10X chromium platform. Data preprocessing used standard Seurat methodology. HD Spot generated optimized classifiers via a custom platform. Network interpretability was achieved using Shapley values. Ontology analysis was performed using Metascape.

RESULTS: HD Spot identified meaningful ontologic signatures across all tested datasets. In the binary case of control versus IFN-B stimulated CD4 + T cells, gene ontologies reflected $\mathrm{Th} 0$ and $\mathrm{Th} 2 \mathrm{~T}$ cell populations, congruent with $\mathrm{T}$ cell activation. In the 9-class case of PBMCs, HD Spot identified meaningful gene networks characteristic of the ground-truth populations using raw feature counts alone. When feature counts are processed into expression values, HD Spot demonstrates increased specificity of top genes and respective ontologies between subpopulations.

CONCLUSION: This work introduces a broadly applicable computational tool for the advanced bioinformatician to decipher complex cellular heterogeneity (e.g., tumors) in an unbiased way. Additionally, HD Spot lowers the barrier for novice bioinformaticists to derive actionable insights from their data.

Keywords: Deep Learning, Single Cell Sequencing, Automated Analyses, Subtyping

FL-060

Moya-Moya disease

Indirect revascularization for moyamoya patients more than 40 years of age

Meng Fai Kuo ${ }^{1}$, Sheng Che $\mathrm{Chou}^{2}$, Ya Fang Chen ${ }^{3}$, Chung Wei $\mathrm{Lee}^{3}$

${ }^{1}$ Division of Neurosurgery, Department of Surgery, National Taiwan University Hospital, National Taiwan University College of Medicine, Taipei City, Taiwan

${ }^{2}$ Division of Neurosurgery, Department of Surgery, National Taiwan University Hospital Yun-Lin Branch, National 
Taiwan University College of Medicine, Yun-Lin County, Taiwan

${ }^{3}$ Department of Medical Imaging, National Taiwan University Hospital, National Taiwan University College of Medicine, Taipei City, Taiwan

OBJECTIVE: This study aims to evaluate the effectiveness of indirect revascularization in moyamoya patients more than 40 years of age.

MATERIAL-METHODS: From January 2013 to June 2019, moyamoya patients more than 40 years of age underwent indirect revascularizations were studied. The indirect revascularization procedures included encephalo-duroarterio-synangiosis, encephalo-myo-synangiosis, and encephalo-pericranio-synangiosis in hypoperfusion area. The pre-operative and post-operative clinical conditions, cerebral angiography, and semi-quantitative time-to-peak (TTP) scoring (ranged 0-14) of the magnetic resonance perfusion study were used to evaluate the revascularization effect.

RESULTS: During the study period, 50 adult moyamoya patients underwent indirect revascularization in our hospital. Seventeen patients ( 27 cerebral hemispheres) more than 40 years of age were included in this study. There were 14 females and 3 males. The mean age was $47.9 \pm 6.4$ years. The preoperative Suzuki stages were I, II, III, IV, V, and VI in $1,1,9,13,0$, and 3 hemispheres, respectively. After a mean follow-up period of $33.5 \pm 20.6$ months, all patients had improvement or stabilization of their clinical conditions. Available post-operative angiography demonstrated Matsushima grading A or B in 11 of 13 hemispheres. The TTP score of all 27 hemispheres improved from $5.0 \pm 3.2$ preoperatively to $11.3 \pm 2.0$ after surgery $(p<0.001)$. Eight of 13 non-operative hemispheres had improvement of perfusion after contralateral revascularization. The complication rate was $14.8 \%$. All were transient except one with mild motor weakness without fully recovery after 15-month follow-up. CONCLUSION: Indirect revascularization can be an alternative treatment with acceptable results and low complication rate in middle-aged patients with moyamoya disease.

Keywords: Moyamoya disease, cerebrovascular disease/ stroke, revascularization

\section{FL-061}

\section{Moya-Moya disease \\ EDAS for Moyamoya disease: How we do it and our experience of 65 operations}

Md Ziauddin, Sudipta Kumar Mukherjee, Sheikh Md. Ekramullah, Dm Arman

National Institute of Neurosciences and Hospital
OBJECTIVE: Moyamoya disease is frequently being diagnosed in Bangladesh in recent years. In our institution we are regularly doing indirect revascularization i.e. Encephalo-duro-arterio-synangiosis(EDAS) for last 6 years for childhood Moyamoya disease. After starting we made some modification of operative technique with time. It's a simple and safe surgery for the serious and devastating disease. As our outcome is quite satisfactory I want to show our operative technique and its outcome.

MATERIAL-METHODS: We operated 59 cases in National Institute of Neurosciences and Hospital, Dhaka, Bangladesh. there were 6 bilateral cases. So total number of cases are 65 in about 6 years time. We follow some definitive steps in our institution which is shown in these paper. Outcome is observed by a retrospective analysis of the results.

RESULTS: The Modified Rankin Scale (mRS) of our series were- $72 \%$ good mRS (mRS 0, 1, 2);26\% moderate mRS (mRS 3, 4, 5) and 2\% death. Three patients had post operative stroke out of 65 surgeries (3/65). One mortality was observed in our cohort. We also face Flap necrosis, CSF leakage.

CONCLUSION: EDAS is a gold standard surgery for childhood Moyamoya disease. We are getting promising result of our operated cases. Only we need is longer follow up of our operated cases and awareness about its diagnosis and management.

Keywords: Moyamoya disease, EDAS, Indirect revascularization

\section{FL-062}

\section{Moya-Moya disease}

Surgical revascularisation (SR) for Paediatric Moya Moya (MM): early outcomes from a surgical apprenticeship and lessons learned

Adikarige Silva $^{1}$, Sanjay Bhate ${ }^{2}$, Vijeya Ganesan ${ }^{2}$, Dominic Nolan Paul Thompson ${ }^{1}$, Greg James ${ }^{1}$

${ }^{1}$ Department of Neurosurgery, Great Ormond Street Hospital, London, UK

${ }^{2}$ Department of Neurology, Great Ormond Street Hospital, London, UK

OBJECTIVE: To analyse early outcomes of surgical revascularisation (SR) for paediatric Moya Moya (MM) during and following a surgical mentorship.

MATERIAL-METHODS: From November 2017, a newlyappointed attending neurosurgeon was mentored in SR for paediatric MM with a view to further service development by a senior attending. The early outcomes for this cohort of 
patients is compared to a historical one of 73 patients at our institution.

RESULTS: We identified 22 children that underwent 36 indirect SRs. Patient demographics (aetiology, mean age at surgery, proportion of patients with anterior and posterior circulatory, and bilateral disease) were almost identical between cohorts.

The first 14 children (23 SRs) underwent encephalo-duroarterio-myo-synangiosis (EDAMS). The last 8 children (13 SRs) underwent EDAMS with incorporation of additional transfixing sutures of the perivascular adventitial cuff to the exposed pial surface (EDAMS + Pial Synangiosis). This change in practice was precipitated by a graft failure in a patient with a previously atrophic brain that developed a post-operative subdural collection with antecedent graft detachment away from the cortical surface.

The first 6 patients had 11 SRs performed jointly by surgeon and mentor, followed by 10 patients with 16 SRs performed independently by the surgeon. The last 6 patients had 9 SRs performed by the surgeon mentoring a senior fellow. There were no differences in outcome between the 3 groups.

There were no peri-operative mortalities and, in comparison to the historical cohort, a similar proportion had a recurrent arterial ischaemic event (AIS) necessitating a 2nd SR (1/22 vs 3/73). Differences between the two cohorts were in length of follow up (median 6.7 months VS 34 months) and the decision to withhold antiplatelet therapy for 10 days preoperatively in the historical cohort.

CONCLUSION: Our early outcomes demonstrate the value of mentorship for safely incorporating new surgeons in developing an existing service.

Keywords: moyamoya, pial synangiosis, EDAMS, training, mentorship

\section{FL-063}

\section{Moya-Moya disease \\ Functional outcomes post encephalo-duro-arteriomyo synangiosis procedure for Moyamoya Disease: A single centre experience}

Neeraj Kalra, Melissa Bautista, Helen Mccullagh, Karen Pysden, Gnanamurthy Sivakumar, Paul Chumas, John Goodden, Atul Tyagi

Department of Paediatric Neurosurgery, Leeds Children's Hospital, Leeds, England

OBJECTIVE: Moyamoya disease is a progressive, occlusive arteriopathy of the intracranial vessels causing an increased risk of stroke. It often results in functional impairment and decrease in quality of life, both in the presence and absence of stroke. Revascularisation is the accepted treatment for patients with symptomatic moyamoya disease, preventing further stroke. In Leeds Children's Hospital we use the encephalo-duro-arterio-myo-synangiosis (EDAMS) technique to facilitate revascularisation. We aim to assess the quality of life outcomes of patients who have undergone operative intervention at our unit for moyamoya disease.

MATERIAL-METHODS: Paediatric patients with operated moyamoya disease from Leeds Children Hospital between February 2009 and January 2019 were included. Patient awaiting primary surgery were excluded. Patients were contacted via telephone and the Paediatric Quality of Life Inventory 4.0 (PedsQL) questionnaire administered via parent proxy. Quality of life outcomes were assessed using the PedsQL questionnaire score, which measures physical, emotional social and school functioning domains. This score was then converted in to a health-related quality of life (HRQOL) score. Scores in each domain and mean scores were compared to normative data.

RESULTS: This study included 11 children, 5 male, median age 6.8 years (range 22 months to 15.5 years) and 9 children underwent bilateral operations. Mean parent proxy PedsQL score was 66 (range 12.5-98.4), with a mean score of 61.9 in physical function, 88.9 in emotional function, 70.9 in social function and 58.7 in school function. This was lower than healthy controls overall and in each individual domain except emotional function which was similar to normative data.

CONCLUSION: Children with moyamoya disease have a lower quality of life than healthy controls within this series. This suggests that children with moyamoya should be offered additional psychosocial support within the community.

Keywords: Moya moya, neurovascular, paediatrics

\section{FL-064}

\section{Moya-Moya disease}

Effectiveness and limitations of indirect anastomosis for pediatric patients with moyamoya disease

Hirofumi Oguma ${ }^{1}$, Taku Uchiyama ${ }^{1}$, Azumi Kaneoka ${ }^{1}$, Kensuke Kawai ${ }^{1}$, Akira Gomi ${ }^{2}$

${ }^{1}$ Department of Neurosurgery, Jichi Medical University, Tochigi, Japan

${ }^{2}$ Department of Pediatric Neurosurgery, Jichi Children's Medical Center Tochigi

OBJECTIVE: Both direct and indirect anastomosis are performed for pediatric patients with moyamoya disease. However, it remains unclear which is more advantageous. We assessed the indications and limitations of indirect anastomosis by evaluating cases treated at our institute. 
MATERIAL-METHODS: We conducted a retrospective study of 38 cerebral hemispheres in 21 patients with moyamoya disease who underwent surgery at our hospital from 2003 to 2018. In this study, outcomes were defined as follows: good, postoperative improvement in symptoms and collateral anastomosis was well developed; fair, symptoms improved postoperatively but development of collateral anastomosis was insufficient; and poor, both postoperative improvement of symptoms and development of collateral anastomoses were insufficient.

RESULTS: Thirty-two hemispheres underwent indirect anastomosis (17 EDAS, 14 EDAS + EMS/EGS, 1 Ribbon method). Age at surgery was 3-21 years (mean, 9.3 years). Six hemispheres underwent both direct and indirect anastomosis (6 STA-MCA + EDMS). The age of these patients at surgery was $7-18$ years (mean, 10.7 years). Of the 32 cases of indirect anastomosis, outcomes in 22 were good, 6 were fair, and 4 were poor. The proportion of good outcomes was $4 / 11(36 \%)$ in the case of patients aged $\leq 7$ years at surgery, and 18/21 (86\%) in cases aged $\geq 8$ years. In the direct and indirect anastomosis group, 5 cases had a good outcome and 1 had a fair outcome. On the other hand, long-term angiographic findings showed that direct anastomoses gradually disappeared as collateral vessels developed from the indirect anastomosis. The incidence of postoperative infarction was $3 / 32(9 \%)$ in the indirect anastomosis group and 2/6 (33\%) in the direct and indirect group.

CONCLUSION: Indirect anastomosis is often sufficient for children age 8 and older. Direct and indirect anastomosis may be more effective, but complications should be noted. It is important that the collateral supply from indirect anastomosis gradually becomes dominant.

Keywords: moya-moya disease indirect anastomosis

\section{FL-065}

\section{Neuro-Oncology \\ CDKN2A deletion in supratentorial ependymoma with RELA alteration indicates a dismal prognosis - a retro- spective analysis of the HIT ependymoma trial cohort}

Stephanie Theresa Jünger $^{1}$, Felipe Andreiuolo ${ }^{1}$, Martin Mynarek $^{2}$, Evelyn Dörner ${ }^{1}$, Anja Zur Mühlen ${ }^{1}$, Natalia Valez-Char ${ }^{1}$, Katja Von Hoff ${ }^{2}$, Stefan Rutkowski ${ }^{2}$, Monika Warmuth-Metz ${ }^{3}$, Rolf Dieter Kortmann ${ }^{4}$, Beate Timmermann ${ }^{5}$, Andre Von Bueren ${ }^{6}$, Torsten Pietsch ${ }^{1}$

${ }^{1}$ Department of Neuropathology, DGNN Brain Tumor Reference Center, University of Bonn, Bonn, Germany

${ }^{2}$ Department of Pediatric Hematology and Oncology, University Hospital Hamburg-Eppendorf, Hamburg, Germany ${ }^{3}$ Institute of Diagnostic and Interventional Neuroradiology, University Hospital Würzburg, Würzburg, Germany
${ }^{4}$ Department of Radiation Oncology, University Hospital Leipzig, Leipzig, Germany

${ }^{5}$ Westdeutsches Protonentherapiezentrum, Essen, Germany ${ }^{6}$ Department of Pediatrics, Obstetrics and Gynecology, Division of Pediatric Hematology and Oncology, University Hospital Geneva, Geneva, Switzerland

OBJECTIVE: Since supratentorial RELA-fusion positive ependymomas are considered a biologically distinct disease, we aimed to identify histological and genetic predictors of outcome in a defined cohort of pediatric patients.

MATERIAL-METHODS: We analyzed 54 RELA ependymomas in children treated according to HIT2000-E protocols. All cases underwent central neuropathological review. Genome-wide copy number alterations were assessed by molecular inversion probe or SNP array. RELA alterations were detected by RT-PCR or sequencing and assessment of nuclear p65-RelA protein. Copy number alteration of the $C D K N 2 A$ (cyclin dependent kinase inhibitor $2 \mathrm{~A}$ ) locus and concordant p16 protein expression were analyzed.

RESULTS: Fifty-two tumors were classified as WHOgrade III (96.3\%) with high mitotic activity in 39 cases (72.2\%), vascular proliferation in $47(87.0 \%)$, necrosis in 43 (79.6\%) and clear cell morphology in 19 (35.2\%). All tumors harbored RELA alterations. Homo- or heterozygous $C D K N 2 A$ deletions were detected in $9(16.7 \%)$ and 14 (25.9\%) cases, respectively. p16 protein expression was lost in all cases with homozygous deletion. Median followup was 5.4 years with 5-years EFS and OS of $74.1 \%$ and 92.6\%. In Kaplan-Meier analysis high mitotic activity was related to shorter EFS $(\mathrm{p}=0.016)$ and clear cell morphology to longer OS ( $\mathrm{p}=0.039) ; C D K N 2 A$ deletion was associated with shorter OS (homozygous deletion, $\mathrm{p}=0.009$; homo-or heterozygous deletion, $\mathrm{p}=0.034$ ). No correlation between $C D K N 2 A$ deletion and high mitotic activity was found but with higher age at diagnosis $(\mathrm{p}=0.001)$.

CONCLUSION: Deletion of CDKN2A occurred in $42.6 \%$ of supratentorial ependymomas with RELA alteration and represented a genetic predictor of worse overall outcome in pediatric patients.

Keywords: Ependymoma, pediatric, supratentorial, genetic, $\{$ CDKN2A $\},\{$ RELA $\}$

\section{FL-066}

Neuro-Oncology

Management of pediatric tectal plate gliomas

Chenran Zhang ${ }^{1}$, Frederick Boop ${ }^{2}$, Ruopin Chen ${ }^{1}$

${ }^{1}$ Department of Pediatric Neurosurgery, Xinhua Hospital, Shanghai, China 
${ }^{2}$ department of Neurosurgery, St Jude Children's Research Hospital, Memphis,TN, USA

OBJECTIVE: Tectal glioma (TG) is a rare low-grade tumor occurring predominantly in the pediatric population. They account for $5 \%$ of brain stem tumors and are most commonly indolent, slow-growing lesions limited to the rostral mesencephalon. Risk factors associated with inferior outcome and long-term sequelae of TG have not been well-documented. MATERIAL-METHODS: We retrospectively reviewed 25 cases of TGs treated at Shanghai Xinhua Hospital from 2008 to 2018. Patient's clinical data were summarized, imaging and pathology specimen were centrally reviewed.

RESULTS: Twenty-five patients with TG were included. The main complaint of admission was headache secondary to obstructive hydrocephalus. Eighteen $(72 \%)$ were male. Median age at diagnosis was 7.8 years (range, 0.01-20.5). Median follow-up was 4.5 years (range, $0.5-17.0$ ). Cerebrospinal fluid diversion was accomplished in all cases, 17 cases with ETV and 8 with VP shunt. Fifteen cases (60\%) underwent tumor-directed surgery: 3 with gross or near total resection, 10 with partial resection, 2 with biopsy. Six patients (24\%) received chemo and radiation therapy and four (16\%) cases was followed without further treatment. Twelve cases were pilocytic astrocytoma and three cases aligned best with diffuse astrocytoma. Lesion $\geq 3 \mathrm{~cm} 2$, contrast enhancement and cystic changes at presentation were risk factors for progression. Among those with tumor tissue available, BRAF duplication (a marker of KIAA1549-BRAF fusion) and BRAF V600E mutation were detected in 25\% and $7.7 \%$, respectively. Median follow-up was 4.5 years. 23 cases were stable without tumor progression. One patient died from the postoperative complication and the other died from tumor relapse.

CONCLUSION: TG is an indolent, chronic disease with unique clinical and molecular profiles. Large size, contrast enhancement and cystic changes are risk factors for progression.

Keywords: tectal plate gliomas, low grade glioma, BRAF V600E mutation

\section{FL-067}

\section{Neuro-Oncology}

Supraorbital Keyhole Approach in pediatric patients: Lessons Learned from 25 Operative Cases

Ricardo Santos De Oliveira, Matheus Fernando Manzolli Ballestero, Vinicius Marques Carneiro

Division of Neurosurgery, Department of Surgery and Anatomy, School of Medicine of Ribeirao Preto University of Sao Paulo, Ribeirao Preto, Sao Paulo, Brazil
OBJECTIVE: The supraorbital keyhole approach through an eyebrow incision has been a potentially less invasive approach as an alternative to the standard pterional craniotomy. We aimed to review procedures for anterior and middle cranial fossa lesions and identify lessons learned from addressing various pathologies through this approach.

MATERIAL-METHODS: We retrospectively reviewed 25 consecutive patients who underwent this approach. We documented patients' age, sex, pathology, clinical results, extent of tumor resection, complications, use of endoscope, and cosmetic results.

RESULTS: Our series addressed a variety of pathologies. Male patients accounted for $64 \%$ of the cohort and mean age was 9.9 years ( 4 months- 17 years). Notably, $80 \%$ of patients underwent resection of extra-axial masses. Craniopharyngioma were the most frequent neoplasm of this series with 10 cases each one. Gross total resection was achieved in $74.4 \%$ of all neoplasic lesions according to postoperative imaging. One child showed a subdural collection. No cerebrospinal fluid fistula or rhinorrhea occurred. Four weeks postoperatively, $95 \%$ of patients demonstrated acceptable cosmetic results. The mean follow-up was 48 months.

CONCLUSION: The supraorbital keyhole approach may be safely used for various lesions within the anterior and middle cranial fossa. The effectiveness and limitations of this approach and possible complications are discussed. This is a valuable approach for selected patients.

Keywords: supraorbital approach, keyhole approach, pediatric neurosurgery, oncology, skull base

\section{FL-068}

\section{Neuro-Oncology \\ Protein signature of pediatric craniopharyngioma}

Luca Massimi ${ }^{1}$, Claudia Martelli ${ }^{2}$, Riccardo Serra ${ }^{3}$, Ilaria Inserra $^{2}$, Diana Valeria Rossetti ${ }^{2}$, Federica Iavarone ${ }^{2}$, Federica Vincenzoni ${ }^{2}$, Massimo Castagnola ${ }^{5}$, Andrea Urbani $^{6}$, Paolo Frassanito ${ }^{1}$, Gianpiero Tamburrini ${ }^{1}$, Claudia Desiderio ${ }^{4}$

${ }^{1}$ Fondazione Policlinico Universitario A. Gemelli IRCCS, Neurochirurgia Pediatrica, Roma, Italy

${ }^{2}$ Istituto di Biochimica e Biochimica Clinica, Università Cattolica del Sacro Cuore, Roma, Italy

${ }^{3}$ Fondazione Policlinico Universitario A. Gemelli IRCCS, Roma, Italy

${ }^{4}$ Istituto di Chimica del Riconoscimento Molecolare, Consiglio Nazionale delle Ricerche, Roma, Italy

${ }^{5}$ Laboratorio di Proteomica e Metabonomica, IRCCS-Fondazione Santa Lucia, Roma, Italy

${ }^{6}$ Area Diagnostica di Laboratorio, Fondazione Policlinico Universitario Agostino Gemelli-IRCCS, Roma, Italy 
OBJECTIVE: Pediatric adamantinomatous craniopharyngioma (pAC) is a major clinical challenge because of the local aggressiveness, which produces neurological symptoms and hormonal dysfunction, and the possible problems in the surgical resection. The pAC cystic component has favored several proteomic studies on the characterization of the intracystic fluid, with the goal to develop therapeutic strategies against this tumor. Poor explorations have been performed so far on the solid part of the tumor, principally investigated by transcriptomics technologies.

MATERIAL-METHODS: The surgical specimens of 7 children affected by pAC have been analyzed by liquid chromatography-mass spectroscopy to preliminary assess the proteomic profile of the tumor by an integrated top-down/ bottom-up approach.

RESULTS: Thymosin beta 4, ubiquitin, calmodulin, S100 proteins, prothymosin $\alpha$ isoform 2 , alpha-defensins $1-4$, and fragments largely belonging to vimentin, hemoglobin, and glial fibrillary acidic protein characterized the intact proteome. In addition, proteins involved in calcium modulation, innate immunity, inflammation, CCKR and integrin signaling, and gonadotropin-releasing hormone receptor pathways were found. A total number of 1798 unique elements were identified by a bottom-up approach, 433 proteins being commonly characterized in $85.7 \%$ of the samples analyzed.

CONCLUSION: The identification of alpha-defensins, previously characterized in pAC intracystic fluid, reinforced the hypothesis on the role for inflammation in the tumor progression. The gene ontology classification of the numerous identified proteins showed the involvement of adherence system, intermediate filaments, and actin cytoskeleton, and the involvement of elements of the Wnt, FGF, and EGFR signaling pathways. These findings suggest a role of these proteins and these pathways in the tumor pathogenesis. These results confirm our previous proteomic data on the PAC intracystic fluid and offer a preliminary overview of the whole tissue protein phenotype, adding new hints towards the comprehension of this still obscure pediatric brain tumor.

Keywords: craniofaringioma, proteomics

\section{FL-069}

\section{Neuro-Oncology}

Stereotactic brainstem biopsies in the management of diffuse intrinsic pontine gliomas

Suresh N Magge ${ }^{1}$, John S Myseros ${ }^{1}$, Lindsay Kilburn ${ }^{2}$, Robert F Keating ${ }^{1}$

${ }^{1}$ Division of Neurosurgery, Children's National Hospital, Washington, DC

${ }^{2}$ Division of Oncology, Children's National Hospital, Washington, DC
OBJECTIVE: Biopsies had been previously avoided in the setting of diffuse intrinsic pontine gliomas (DIPG's), given high morbidity and lack of therapeutic options. In recent years, stereotactic biopsies have become more common, often done as part of clinical trials. We present our series of stereotactic biopsies of DIPG's.

MATERIAL-METHODS: This was an IRB-approved retrospective review of patients at Children's National Hospital (Washington, DC) from 2015 to 2019 who underwent stereotactic biopsy when DIPG was suspected. Charts were reviewed for clinical and radiographic information.

RESULTS: There were 23 patients who underwent stereotactic brainstem biopsies ( 11 male, 12 female). All surgeries were done via a trans-middle cerebellar peduncle approach, using frameless navigation (10 left side, 13 right side). Mean age at surgery was 7.68 years (range 3.24 years to 25.95 years). The most common presenting symptoms were gait instability (18/23), facial weakness (11/23), and visual complaints (10/23). All biopsies except one were done after diagnosis and before radiation treatment (mean time from diagnosis to upfront biopsy was 7.6 days). One biopsy was done after radiation treatment. Pathology showed 22/23 to be DIPG's with the following WHO grades: Grade II: 1, Grade III: 1, Grade IV: 19, unspecified: 1. Of DIPG samples that were tested for H3K27M mutation, 19/19 were positive for the mutation. 1/23 was not a DIPG, and was called hypercellular glial tissue. 3 patients had postoperative morbidities ( 2 facial weakness, 1 paresthesias). There were no surgical mortalities. We also show a novel but simple method of merging CT and MRI scans with frameless navigation to improve accuracy.

CONCLUSION: Stereotactic biopsies of DIPG's can be done safely and effectively. Accuracy of frameless navigation for lesions in the posterior fossa can be challenging, and we discuss a novel but simple method to improve accuracy of frameless navigation in the posterior fossa.

Keywords: diffuse intrinsic pontine glioma, DIPG, biopsy, brain tumor, stereotactic

\section{FL-070}

\section{Neuro-Oncology}

Optic pathway tumor in children: toward a new classification for neurosurgical use

Stella Irene $^{1}$, Helleringer Mathieu ${ }^{1}$, Joud Anthony ${ }^{1}$, Chastagner Pascal ${ }^{2}$, Remen Thomas ${ }^{3}$, Klein Olivier ${ }^{1}$

${ }^{1}$ Pediatric Neurosurgical Unit, Nancy Regional University Hospital, University of Lorraine, France

${ }^{2}$ Pediatric Onco-hematology Department, Nancy Regional University Hospital, University of Lorraine, France 
${ }^{3}$ Methodologic, Data-management and Statistics Unit, Nancy Regional University Hospital, University of Lorraine, France

OBJECTIVE: Optic pathway tumors (OPT) represent a challenge for pediatric neurosurgeons. Role of surgery is debated due to the high risk of iatrogenic damage, and in lasts decades it lost its importance in favor of chemotherapy. However, in some cases surgery is necessary to make biomolecular and histological diagnosis, to manage intracranial hypertension $(\mathrm{IH})$ and to cooperate with medical therapies in controlling tumor relapse. With the aim to standardize selection of surgical OPT cases, we propose a simple, practical and reproducible classification.

MATERIAL-METHODS: We retrospectively analyzed data of 38 patients with OPT treated at our institution (1990-2018). After careful analysis of MRI images, we describe a new classification system. Group 1: lesion limited to one or both optic nerve(s). Group 2: chiasmatic lesions extending minimally to hypothalamus. Group 3: hypothalamo-chiasmatic exophitic lesions invading the third ventricle; they can be further divided on the base of concomitant hydrocephalus. Group 4: hypothalamo-chiasmatic lesions extending widely in lateral direction, toward the temporal or the frontal lobes. Patients' data and adopted treatment are reported and analyzed, also depending on this classification. RESULTS: Twenty children were operated on for treatment of OPT during the study period. Permanent clinical impairment was noted in $5(25 \%)$ of operated patients, while visual improvement was noted in 1 patient. OS rate was $100 \%$ at 5 years, with a median follow up of 9 years (ranging from 2 to 23). Prevalence of intracranial hypertension and proportion of first-line surgical treatment decision were significantly higher in groups 3-4 compared to groups 1-2 ( $\mathrm{p}<0.001$ for both tests).

CONCLUSION: Surgery can offer a valuable therapeutic complement for OPT without major risk of iatrogenic damage. Surgery is indispensable in cases presenting with $\mathrm{IH}$, as in groups 3 and 4 lesions. Eligibility of patients to surgery can be based on this new classification system.

Keywords: Optic Pathway tumor, glioma, pediatric neurosurgery, hypothalamus, chiasmatic tumors

\section{FL-071}

\section{Neuro-Oncology \\ Optimising surgical management of optic pathway gliomas}

Ciaran Scott Hill, Kristian Aquilina

Great Ormond Street Hospital, London, UK
OBJECTIVE: Optic pathway gliomas (OPG) account for 5\% of paediatric brain tumours. Most are WHO grade 1 pilocytic astrocytomas, and one third arise in the context of NF1. OPGs are associated with long-term morbidity related to hydrocephalus, visual impairment, and endocrine and hypothalamic dysfunction. Management is highly individualized; traditionally surgery has been used to treat hydrocephalus, decompress cysts, confirm diagnosis and reduce tumour bulk when other treatments have failed. Their natural history and the accumulation of morbidity, and the influence of surgery on both, remain unclear. The objective of the study is to identify the long-term outcome of surgery for OPGs, and to evaluate how the recent availability of molecular therapies, such as MEK pathway inhibitors, have influenced surgical management.

MATERIAL-METHODS: The department of neurosurgery at Great Ormond Street Hospital has maintained an operative and referral database of all cases since the 1980s. This allowed us to extract a comprehensive database of patients with OPGs. A retrospective analysis of these cases allowed us to correlate key factors relating to outcomes following surgical management of OPGs.

RESULTS: We collated data from over 100 patients with OPG and present results relating to patient demographics (age, sex, presenting symptoms), vision, tumour characteristics on presentation (size, location, histology/molecular profile, NF status), and treatment (operation types and details + non-surgical interventions). These are correlated to a range of short and long-term patient outcomes including overall and progression free survival, neurological/visual/ endocrine/hypothalamic dysfunction, and cerebrospinal fluid diversion rates.

CONCLUSION: The data from this study allows a discussion on which patients benefit most from surgery, the disease stage at which surgery is most effective, as well as the longterm outcome of surgery.

Keywords: Optic pathway glioma, surgery, outcomes, management

\section{FL-072}

\section{Neuro-Oncology}

A New Consideration of Intra - Operative Frozen Section in Management of Pineal Region Tumor

Muhammad Reza Arifianto, Muhammad Fakhri Raiyan Pratama, Wihasto Suryaningtyas, Muhammad Arifin Parenrengi

Department of Neurosurgery, Dr. Soetomo General Hospital / Faculty of Medicine - Universitas Airlangga, Surabaya, Indonesia 
OBJECTIVE: The pineal region is a rare site for primary tumors of the central nervous system and it often leads to devastating neurosurgical complication related to high morbidity, high mortality and high disability. Complex site of anatomy and surgically intimidating environment need comprehensive management strategy to get optimum result. Most of current literatures rely from the serum marker result. Current study aims to give better insight about the use of frozen section to adopt new consideration in proposed guideline on our experience in single institution.

MATERIAL-METHODS: The authors performed a retrospective analysis between January 2012 and March 2019 involving patients whose tumors met the definition criteria of Pineal region tumor and underwent surgery in Dr. Soetomo Academic General Hospital. The clinical course, management, surgical approach and histopathology results were recorded.

RESULTS: 19 patients were included in the study with the average age of patients was 9,89 yo (1-18 yo) with 2:1 preponderance of male. Out of 13 patients came with hydrocephalus underwent ventriculoperitoneal shunt (single/double staged surgery). From histopathology result, Germinoma (8/19) was the most frequent case followed by NGGCT (3/19), pineal parenchymal tumor (2/19), and others (Pilocytic Astrocytoma, Atypical Teratoid/ Rhabdoid Tumor, and Ependymoma). Surgical procedure was done with different approach based on patient's individual condition, mostly using Occipital Transtentorial Approach with the result of nine patients underwent only biopsy, five with partial-subtotal resection and total resection in five patients. CONCLUSION: Regardless of surgical approach, decisionmaking in the management of pineal region tumors remains the same. Frozen section during operation in combination with serum/cerebro-spinal fluid marker can determine the goal of surgical resection to avoid high mortality and morbidity. The combination might alleviate the uncertainty of determining the follow-up management. However, further studies are warranted to confirm our findings to form a recommendation or protocol as standardized approach for pineal region tumor management.

Keywords: Pineal region tumor, frozen section, serum marker, germinoma, outcome

\section{FL-073}

\section{Neuro-Oncology}

Non-operative management of suspected calvarial Langerhans Cell Histiocytosis in children - a prospective multicenter study

\author{
Paul Steinbok $^{1}$, Alex Cheong ${ }^{1}$, David Dix ${ }^{3}$, David I \\ Sandberg ${ }^{2}$
}

${ }^{1}$ Division of Neurosurgery, Department of Surgery, British Columbia Children's Hospital, Vancouver, BC, Canada; Department of Surgery, University of British Columbia, Vancouver, BC, Canad

${ }^{2}$ Departments of Pediatric Surgery and Neurosurgery, Children's Memorial Hermann Hospital and Mischer Neuroscience Institute, Houston, Texas, U.S.A

${ }^{3}$ Division of Hematology and Oncology, Department of Pediatrics, British Columbia Children's Hospital, Vancouver, BC, Canada

OBJECTIVE: The usual treatment of solitary calvarial Langerhans Cell Histiocytosis (C-LCH) is surgical resection, but two studies have reported spontaneous resolution of C-LCH. This multicenter study evaluated outcomes of children with solitary C-LCH managed with observation only. MATERIAL-METHODS: Prospective multicenter study of children with suspected solitary C-LCH based on typical clinical and radiological features, supplemented with bone scans and/or skeletal survey. Management was observation only. For safety reasons, rescue intervention (surgery) was prescribed if the lesion increased rapidly, continued increasing at 2 months, was not stabilizing or getting smaller at 3 months or if pain was unacceptable to patient. Patients, who declined participation in the observational study and opted for surgery, were also followed. Outcomes were determined at 1 year.

RESULTS: 29 children, mean age 11.5 years were enrolled from 8 centers. Observation only was done in 18,15 of whom reached 1 year follow-up, with complete regression and reconstitution of the bony cranial defect in 100\%. 11 patients opted for surgery as initial treatment, and in 10, where pathology was available, LCH was confirmed. At initial consultation, lesion size ranged from 0 (disappeared already) to $75 \mathrm{~mm}$. For the observational arm, all lesions decreased or disappeared by 2 months. The average number of radiologic investigations was 4.4 for the observational versus 6.8 for the surgical group.

CONCLUSION: Observation may be the preferred initial management of probable solitary C-LCH. Concerns about the rare misdiagnosis could be addressed by application of specific criteria to initiate surgical intervention.

Keywords: Cranial defect, skull tumor, treatment, diagnosis, histiocytosis, eosinophilic granuloma

\section{FL-074}

\section{Neuro-Oncology}

Epidemiology of pediatric primary brain tumors: A fiveyear review from a single Indonesian tertiary health-care center 
Muhammad Arifin Parenrengi, Yunus Kuntawi Aji, Wihasto Suryaningtyas

Pediatric Neurosurgery Division, Department of Neurosurgery Faculty of Medicine Universitas Airlangga/dr. Soetomo General Academic Hospital, Surabaya, Indonesia

OBJECTIVE: There are several reports regarding the epidemiology of pediatric primary brain tumors. However, little is known about the profile in Indonesia, particularly in Surabaya. The authors report the results of epidemiological analysis of a retrospective review of childhood primary brain tumors in a single tertiary health-care center.

MATERIAL-METHODS: We conducted a retrospective study of all cases of primary brain tumors from 1 to 18 years diagnosed at the Department of Neurosurgery Faculty of Medicine Universitas Airlangga/dr. Soetomo General Academic Hospital, Surabaya, Indonesia between January 2015 and December 2019.

RESULTS: One hundred and fifty-one primary brain tumors were reviewed with a mean of 30.2 cases per year. According to the gender, $56.9 \%$ (86 cases) of the tumors occurred in males. The mean age was 9.24 years. Of all the tumors, $55 \%$ were situated in the supratentorial compartment. In the infratentorial compartment, 78\% (53 cases) of tumors are located in the cerebellum. In the supratentorial compartment, $35 \%$ (29 cases) of tumors are located in the sellar/suprasellar region. The most common types of tumors diagnosed were pilocytic astrocytoma and medulloblastoma together accounting for half of the cases (50\%, 75 cases) $(26.5 \%$ and $23.5 \%$ respectively), followed by glioblastoma multiforme (GBM) (6\%, 9 cases), ependymoma (6\%, 9 cases), germinoma (5.3\%, 8 cases), craniopharyngioma ( $4.6 \%, 7$ cases), ganglioglioma (3.3\%, 5 cases), supratentorial central nervous system (CNS) embryonal tumor/primitive neuroectodermal tumor (PNET) (2.6\%, 4 cases), and meningioma (2.6\%, 4 cases). The other tumors represent $20.2 \%$.

CONCLUSION: This retrospective study on 151 consecutive patients established a baseline of pediatric primary brain tumors pattern on the basis of histopathological experience at a tertiary health-care center in Surabaya, Indonesia.

Keywords: Epidemiology, Pediatric, Primary brain tumor, Indonesia, Histopathology, Neurosurgery

\section{FL-075}

\author{
Neuro-Oncology \\ Blood-brain barrier disruption and enhanced drug \\ delivery with an unfocused ultrasound device in the \\ brainstem - A preclinical study in healthy and tumor- \\ bearing mice
}

Kévin Beccaria $^{1}$, Ludivine Le Dret $^{2}$, Emmanuèle Lechapt $^{3}$, Manon Lancien ${ }^{2}$, Alexandre Plessier ${ }^{2}$, Guillaume Bouchoux ${ }^{4}$, Michael Canney ${ }^{4}$, Atmane Seck ${ }^{5}$, Lionel Mercier $^{5}$, Karine Ser Le Roux ${ }^{6}$, Stéphanie Puget ${ }^{1}$, Lauriane Goldwirt ${ }^{7}$, Angelo Paci ${ }^{5}$, Alexandre Carpentier ${ }^{8}$, David Castel $^{2}$, Jacques Grill ${ }^{9}$

${ }^{1}$ Department of Pediatric Neurosurgery, Necker-Enfants Malades Hospital, Université de Paris, Paris, France

${ }^{2}$ UMR8203 "Vectorologie et Thérapeutiques Anticancéreuses," CNRS, Gustave Roussy, Université Paris-Sud, Université Paris-Saclay, Villejuif, France

${ }^{3}$ Department of Neuropathology, GHU Paris-Neurosciences, Sainte-Anne Hospital 1, rue Cabanis, 75,014, Paris, France ${ }^{4}$ CarThera, Institut du Cerveau et de la Moelle épinière (ICM), Paris, France

${ }^{5}$ Pharmacology department, Biology and Medical Pathology department, Gustave Roussy et Université Paris Saclay, Villejuif, France

${ }^{6}$ Gustave Roussy Comprehensive Cancer Center, 94,805 Villejuif, France; Université Paris Saclay; Plate-forme d'évaluation préclinique, UMS 23/3655, 94,805 Villejuif, France

${ }^{7}$ Pharmacology Department, Saint-Louis Hospital, AP-HP, 1 Avenue Claude Vellefaux, F-75010, Paris, France; INSERM UMR S976, Université de Paris, Paris, France

${ }^{8}$ Department of Neurosurgery, Sorbonne Université, UPMC Univ Paris 06, Assistance Publique-Hôpitaux de Paris (APHP), Hôpitaux Universitaires La Pitié-Salpêtrière, Paris, France ${ }^{9}$ Department of Pediatric Oncology, Gustave-Roussy, Université Paris-Sud, Université Paris-Saclay, Villejuif, France

OBJECTIVE: Diffuse Intrinsic Pontine Gliomas (DIPGs) have a dismal prognosis. The blood-brain barrier (BBB) plays a major role in the failure of medical treatments by inhibiting adequate penetration of therapeutic agents in the infiltrated tissue. We assessed the feasibility and safety of BBB disruption (BBBD) with Low Intensity Pulsed Ultrasound (LIPU) in the brainstem of healthy and tumor-bearing mice with an unfocused ultrasound device in association with microbubbles and evaluated drug delivery to the brainstem.

MATERIAL-METHODS: BBBD was assessed in healthy nude mice and in cell-derived orthotopic xenograft (CDOX) models of DIPG. Sonications were performed with the SonoCloud ${ }^{\circledR}$ device at a frequency of $1.05 \mathrm{MHz}$ and acoustic pressures varying from 0.3 to $0.5 \mathrm{MPa}$. BBBD was evaluated with Evans blue dye and $70 \mathrm{kDa}$ fluorescent dextrans. Clinical parameters were monitored during sonications. Histological analysis of the brainstem parenchyma and tumor tissue was performed. The pharmacokinetics of irinotecan and panobinostat in plasma and brainstem were assessed, 
with or without BBBD, in tumor-bearing mice and healthy nude mice, respectively.

RESULTS: We observed a significant blue coloration and fluorescence in healthy brain and tumor tissue, demonstrating that BBBD was obtained with the SonoCloud ${ }^{\circledR}$ device. Sonications at 0.3 MPa were well-tolerated without induced histological lesions. In CDOX mice, irinotecan concentrations were significantly higher in sonicated pons at 30 and 120 min after treatment, compared to non-sonicated pons (concentration ratios 4.3, p $<0.001$ and 2.8, $\mathrm{p}<0.05$, respectively, two-way ANOVA). A significant increase in panobinostat concentration in sonicated pons compared to nonsonicated pons of healthy nude mice was observed three hours after treatment (concentration ratio 1.41, p $<0.05$, two-way ANOVA).

CONCLUSION: Safe LIPU-induced BBBD was obtained in brainstem of healthy and tumor-bearing mice with the SonoCloud ${ }^{\circledR}$ device. US-BBBD allowed for increased delivery of irinotecan and panobinostat in murine DIPG CDOX model and healthy brainstem.

Keywords: DIPG, Brainstem, Blood-Brain barrier, Ultrasound, LIPU, Panobinostat

\section{FL-076}

\section{Neuro-Oncology}

Usefulness of bevacizumab in maintaining quality of life at the time of diffuse intrinsic pontine glioma relapse

\author{
Akira Gomi ${ }^{1}$, Taku Uchiyama ${ }^{2}$, Hirofumi Oguma ${ }^{2}$, Takashi \\ Yamaguchi $^{2}$, Kensuke Kawai ${ }^{2}$ \\ ${ }^{1}$ Department of Pediatric Neurosurgery, Jichi Children's \\ Medical Center Tochigi, Jichi Medical University, Tochigi, \\ Japan \\ ${ }^{2}$ Department of Neurosurgery, Jichi Medical University, \\ Tochigi, Japan
}

OBJECTIVE: Even in the age of molecular diagnosis, diffuse intrinsic pontine glioma (DIPG) is still a dismal disease, and there is no effective treatment. The usefulness of bevacizumab for DIPG relapse is reported.

MATERIAL-METHODS: The treatment and outcomes of 10 patients with DIPG who were treated at our institute since 2001 were retrospectively reviewed. All patients were diagnosed with DIPG by MRI imaging and underwent radiation therapy first. Chemotherapy was performed in combination with radiation therapy in 4 cases, and 3 of them did not receive chemotherapy at the time of relapse (Untreated Group). In 7 cases, chemotherapy was performed at the time of relapse with ACNU/vincristine or interferon beta (Other Treatment Group), and 2 cases with bevacizumab (Bv
Group). The change in the Karnofsky Performance Status Scale (KPS) from the time of relapse was compared.

RESULTS: The average overall survival (OS) for all 10 cases was 10.0 months, 8.1 months in the Untreated Group, 9.5 months in the Bv Group, and 11.4 months in the Other Treatment Group. No prolongation of OS by bevacizumab was observed. However, it was only in the Bv Group that the KPS increased from the time of relapse. Comparison of the KPS at the time of relapse and the KPS after 4 months showed that the $\mathrm{Bv}$ Group remained unchanged or increased from 80 to 90 , while the Untreated Group decreased by 60-100, and the Other Treatment Group also decreased by 20-50. In the Other Treatment Group, hospitalization was required for treatment, and side effects of bone marrow suppression were observed. However, in the Bv Group, outpatient treatment was possible, there were no side effects, and all could be observed at home.

CONCLUSION: From the above results, bevacizumab appears useful for palliative treatment for maintaining quality of life after DIPG relapse.

Keywords: bevacizumab, diffuse intrinsic pontine glioma, quality of life

\section{FL-077}

\section{Neuro-Oncology \\ H3K27M mutation revertance restores H3K27 trimeth- ylation in diffuse midline glioma}

Cody Lee Nesvick ${ }^{1}$, Charles A. Day ${ }^{2}$, Liang Zhang ${ }^{1}$, Edward H Hinchcliffe $^{2}$, David J. Daniels ${ }^{1}$

${ }^{1}$ Department of Neurologic Surgery, Mayo Clinic, Rochester, MN, USA

${ }^{2}$ Department of Cell Biology, Hormel Institute, Austin, MN, USA

OBJECTIVE: Diffuse midline glioma (DMG) is a lethal brain tumor that typically occurs in children. Numerous studies have demonstrated the central role of the H3K27M mutation and secondary loss of H3K27 trimethylation (H3K27me3) in DMG tumorigenesis. Understanding how the H3K27M mutation alters the epigenetic landscape of the cell is necessary for revealing molecular targets that are critical to tumorigenesis.

MATERIAL-METHODS: To investigate the epigenetic effects of H3K27M mutation in DMG, we developed revertant DMG cell lines with the mutant methionine residue reverted to wildtype (i.e., M27K). Revertant cells were analyzed for epigenetic changes and phenotypic differences in vitro and in vivo. 
RESULTS: H3M27K DMG cells grew in culture but displayed diminished proliferative capacity. H3M27K cells demonstrated total loss of H3K27M expression and restored trimethylation of H3K27 and H3K4. Furthermore, consistent with the hypothesis that the H3K27M mutation impacts H3 phosphorylation via expression of Aurora Kinase during mitosis, H3M27K cells demonstrated reduced expression of both Aurora Kinase A and phosphorylation of $\mathrm{H} 3$ serine residues 10 and 28. In line with the critical role of $\mathrm{H} 3 \mathrm{~S} 10$ phosphorylation in chromatin segregation, H3M27K cells also demonstrated restored chromosome segregation compared to H3K27M cells. In vivo data will be discussed.

CONCLUSION: Revertance of the H3K27M mutation reduces tumorigenesis in DMG. Isogenic H3M27K cells have reversal of key epigenetic changes associated with oncogenesis in DMG. The revertant H3M27K DMG model is a useful tool to investigate the downstream epigenetic reprogramming specific to $\mathrm{H} 3 \mathrm{~K} 27 \mathrm{M}$ mutation in these tumors.

Keywords: Diffuse midline glioma, H3K27M, histone, DIPG

\section{FL-078}

\section{Neuro-Oncology}

An Organotypic Chunk Culture Technique To Study Disease Mechanism and Develop Targeted Therapeutics For Pediatric Adamantinomatous Craniopharyngioma

Trinka Vijmasi ${ }^{1}$, Eric Prince ${ }^{1}$, Astrid Hengartner ${ }^{1}$, Susan Staulcup $^{1}$, Andrea Griesinger ${ }^{2}$, Andrew Donson ${ }^{2}$, Nicholas Foreman $^{2}$, Ahmed Gilani ${ }^{3}$, Todd Hankinson ${ }^{1}$

${ }^{1}$ Department of Neurosurgery, University of Colorado Denver, Anschutz Medical Campus, Aurora, CO, 80,045, USA ${ }^{2}$ Department of Pediatrics, University of Colorado Anschutz Medical Campus, Aurora, CO, 80,045, USA

${ }^{3}$ Department of Pathology, University of Colorado Denver, Anschutz Medical Campus, Aurora, CO, 80,045, USA

OBJECTIVE: Advances in the treatment of Adamantinomatous Craniopharyngioma (ACP) face challenges with translation to clinical study due to the absence of robust culture models of the disease. We optimized a technique for culturing human ACP tissue in an organotypic chunk culture format that retains the tumor microenvironment for a duration sufficient to evaluate potential targeted therapeutics. MATERIAL-METHODS: Intraoperatively collected tumor tissue from pediatric ACP was cut into volumes of approximately $3 \mathrm{~mm}^{3}$ and rested over a semi-permeable insert placed in the wells of a 6 -well plate. Specimens were cultured in (1) Control media, media containing (2) Tocilizumab, (3) Trametinib, and (4) combination of Tocilizumab and Trametinib, for 24 and $96 \mathrm{~h}$. Specimens were harvested for paraffin embedding, protein and gene expression assays. Supernatants were collected to assay secreted components. Immunohistochemistry for Pan-Cytokeratin, betaCatenin, cleaved Caspase-3, Ki-67, and Phospho-ERK was performed.

RESULTS: Characteristic histologic features of ACP with epithelial cells with palisading nuclei, wet keratin and ghost cells were identified. Tumor sections were markedly positive for Pan-CK and Beta-Catenin. Ki-67 and cleaved Caspase-3 were restricted to a small fraction of cells. Phospho-ERK staining was observed mostly in the peripheral palisading epithelium and it appeared to be decreased with Trametinib treatment. The response to experimental drugs shall be further characterized using assays of gene expression and protein expression in tissue and secreted components.

CONCLUSION: The organotypic chunk culture technique appears to maintain the viability and integrity of ACP tumors for several days. The response of tumors to experimental drugs can be investigated. This may serve as a suitable model for pre-clinical studies to develop targeted therapeutics for pediatric ACP.

Keywords: Adamantinomatous Craniopharyngioma, Chunk Culture, Trametinib, Tocilizumab

\section{FL-079}

\section{Neuro-Oncology \\ Cyst Fluid Cytokines May Promote Epithelial-to- Mesenchymal Transition In Pediatric Adamantinoma- tous Craniopharyngioma}

Trinka Vijmasi ${ }^{1}$, Eric Prince ${ }^{1}$, Astrid Hengartner ${ }^{1}$, Chibueze Agwu $^{2}$, Susan Staulcup ${ }^{1}$, Maryna Pavlova ${ }^{3}$, Andrea Griesinger $^{4}$, Andrew Donson ${ }^{4}$, Kathleen Dorris ${ }^{4}$, Michael Handler $^{5}$, Todd Hankinson ${ }^{1}$

${ }^{1}$ Department of Neurosurgery, University of Colorado Denver, Anschutz Medical Campus, Aurora, CO, 80,045, USA ${ }^{2}$ College of Arts and Sciences, Washington University in St. Louis, St. Louis, MO, 63,130

${ }^{3}$ Gates Center for Regenerative Medicine, Department of Dermatology, University of Colorado Denver, Anschutz Medical Campus, Aurora, CO, USA

${ }^{4}$ Department of Pediatrics, Children's Hospital Colorado, Aurora, CO, USA

${ }^{5}$ Department of Neurosurgery, Children's Hospital Colorado, Aurora, CO, USA

OBJECTIVE: Despite poor clinical outcomes, no targeted therapies have been established for the treatment of Adamantinomatous Craniopharyngioma (ACP). The only known genetic aberration is a mutation in CTNNB1 that results in 
the nuclear accumulation of beta-catenin. Nuclear betacatenin is an established inducer of Epithelial-to-Mesenchymal Transition (EMT). ACP cyst fluid is enriched with pro-inflammatory and SASP cytokines, many of which are also directly implicated in EMT. We sought to investigate the role of EMT in ACP pathology.

MATERIAL-METHODS: Normal human epithelial cells were cultured and treated with ACP cyst fluid (10\%) for 1 , 2, 4 and 8 days. Cell morphology was monitored by live-cell brightfield microscopy. The expression of EMT associated genes, ZEB1, ZEB2, SNAI-1, SLUG, TWIST, E-Cadherin, Beta-Catenin and Vimentin was determined by RT-qPCR. RESULTS: ACP cyst fluid treated epithelial cells were markedly transformed into long, spindle-shaped cells. ACP cyst fluid treatment resulted in the progressive upregulation of $\mathrm{ZEB} 2$ over 8 days $(\mathrm{RQ}=12.0 ; \mathrm{P}<0.01)$, the progressive upregulation of SNAI-1 over 4 days $(\mathrm{RQ}=5.1 ; \mathrm{P}<0.05)$ and upregulation of Vimentin $(\mathrm{RQ}=2.2 ; \mathrm{p}<0.01)$, identified only on Day 8.

CONCLUSION: ACP cyst fluid can induce EMT-like changes in normal human epithelial cells. In conjunction with the frequency of beta-catenin mutation in ACP, it is possible that EMT plays a crucial role in the pathology of ACP. Understanding ACP pathology in the context of the EMT paradigm may aid the development of new targeted therapeutics.

Keywords: Epithelial-to-Mesenchymal Transition, Adamantinomatous Craniopharyngioma, ZEB2, SNAI-1, beta-catenin

\section{FL-080}

\section{Neuro-Oncology \\ Endoscopic Endonasal Skull-base Surgery in the Pediat- ric Population: A single centre experience}

Hanan Algethami, Doron Sommer, Olufemi Ajani, Keseva K Reddy, Blake A Yarascavitch

McMaster University

OBJECTIVE: There are number case series of pediatric endonasal skull-based surgery in the literature. We aim to describe the recent experience of the transition to endonasal surgery at McMaster Children's Hospital.

MATERIAL-METHODS: A retrospective case series review of endonasal skull-based operations from 2015-2020 was performed from a single author case $\log$ and reviewed for patient demographics, type of operation performed, pathology and peri-operative complications.

RESULTS: A total of 23 operations in 16 patients were performed during this period. Sixty-nine percent were male. Average age at operation was 11.04 years (range
2.98 - 17.46) and there were two peri-operative complications (one CSF leak, one acute hydrocephalus). Pathologies included craniopharyngioma (5), juvenile nasal angiofibroma (5), germinoma (3), meningioma (2), fibrous/osseous lesions (3), lymphoma (2), abscess (1), sarcoma (1) and encephalocele (1). Operations were classified as resections/debulking in 15 (68\%), biopsy in 5 (23\%), optic nerve decompression in $1(5 \%)$, and encephalocele repair in $1(5 \%)$ and CSF leak repair in $1(5 \%)$.

CONCLUSION: The present series describes our recent experience with endonasal skull-based surgery in the pediatric population. This adds to the growing available literature demonstrating the feasibility of this type of operation for a variety of pathologies despite traditional concerns regarding adequate access.

Keywords: endonasal, endoscopic, skull base, craniopharyngioma, germinoma

\section{FL-081}

\section{Neuro-Oncology \\ Quantitative MR Imaging Features Associated With Unique Transcriptional Characteristics in Pediatric Ada- mantinomatous Craniopharyngioma: A Potential Guide For Therapy}

David Mirsky $^{1}$, Eric Prince ${ }^{2}$, Susan Staulcup ${ }^{2}$, Astrid Hengartner $^{2}$, Trinka Vijmasi ${ }^{2}$, Todd Hankinson ${ }^{2}$

${ }^{1}$ Department of Radiology, University of Colorado Anschutz Medical Campus, Aurora, USA

${ }^{2}$ Department of Neurosurgery, University of Colorado Anschutz Medical Campus, Aurora, USA

OBJECTIVE: Adamantinomatous Craniopharyngioma (ACP) is associated with clinical heterogeneity, with some patients suffering substantial morbidity and others having a relatively straightforward clinical course. Neither radiographic nor genetic characteristics that explain this variability have been identified.

MATERIAL-METHODS: Through the Advancing Treatment for Pediatric Craniopharyngioma (ATPC) consortium we accumulated preoperative MRIs and tumor RNA for 50 unique ACP patients. MRIs were assessed quantitatively for 28 different features and analyzed using Multiple Factor Analysis (MFA) and optimal clustering was determined via maximization of Bayesian Information Criterion (BIC). Following bulk RNAseq, differential expression and pathway enrichment were performed using standard methodologies (i.e., DESeq2 and GSEA).

RESULTS: MRI features were well represented in the first 3 dimensions of MFA (variance explained $=67.32 \%$ ); specifically tumor/cyst size, ventricular size, and cyst fluid 
diffusivity. Using this three-way axis, we identified 3 patient subgroups. Transcriptional differences between these subgroups indicated one group was enriched for DNA damage response and MYC related pathways, one group enriched for $\mathrm{SHH}$, and one group enriched for $\mathrm{WNT} / \beta$-catenin and EMT-related pathways.

CONCLUSION: This preliminary work suggests that there may be unique gene expression variants within ACP, which may be identified preoperatively using easily quantifiable MRI parameters. These radiogenomic signatures could provide prognostic information and/or guidance in the selection of antitumor therapies for children with ACP.

Keywords: Adamantinomatous Craniopharyngioma, Quantitative Imaging, Imaging Genomics, Machine Learning

\section{FL-082}

\section{Neuro-Oncology}

Adamantinomatous Craniopharyngioma Resides Outside the Blood Brain Barrier

Eric Prince $^{1}$, Trinka Vijmasi ${ }^{1}$, Jennifer Mcwilliams ${ }^{2}$, Astrid Hengartner ${ }^{1}$, Susan Staulcup ${ }^{1}$, Nicholas Foreman ${ }^{3}$, Kimberly Jordan ${ }^{2}$, Kathleen Dorris ${ }^{3}$, Lindsey Hoffman ${ }^{4}$, Todd Hankinson ${ }^{1}$

${ }^{1}$ Department of Neurosurgery, University of Colorado Anschutz Medical Campus, Aurora, USA

${ }^{2}$ Department of Immunology and Microbiology, University of Colorado Anschutz Medical Campus, Aurora, USA

${ }^{3}$ Department of Neurooncology, Children's Hospital Colorado, Aurora, USA

${ }^{4}$ Department of Neurooncology, Pheonix Children's Hospital, Pheonix, USA

OBJECTIVE: Adamantinomatous craniopharyngioma (ACP) is a devastating skull-base tumor believed to derive from epithelial remnants of the primordial craniopharyngeal duct (Rathke's pouch), which gives rise to the anterior pituitary gland. Genetically engineered mouse models of ACP demonstrate that perturbation of the fetal anterior pituitary can generate tumors analogous to ACP. Clinical and preclinical data indicate that IL-6 blockade may contribute to ACP tumor control, with the most common agent being the humanized monoclonal antibody, tocilizumab. This agent demonstrated poor blood-brain barrier (BBB) penetration in primates. We present findings from two children enrolled on a phase 0 clinical trial (NCT03970226) of a single dose of preoperative intravenous tocilizumab prior to resection of newly diagnosed ACP.

MATERIAL-METHODS: Blood samples were obtained at multiple timepoints. Serum was isolated via ficoll separation. Tumor tissue and cyst fluid were obtained 4-6 h following the single IV dose of tocilizumab. Tissue was snap-frozen. Tumor was homogenized in RIPA buffer. Free tocilizumab in serum, cyst fluid, and tumor tissue was measured using enzyme-linked immunosorbent assay (ELISA) against a standard curve.

RESULTS: Both patients in this trial demonstrated clinically relevant levels of tocilizumab $(\geq 4 \mu \mathrm{g} / \mathrm{mL})$ in serum, cyst fluid, and tumor tissue, compared to undetectable levels in control samples.

CONCLUSION: ACP resides outside BBB protection. In addition to demonstrating the feasibility of systemic delivery of tocilizumab, these findings indicate that other large molecules, including those known to have poor BBB penetration, may be systemically delivered as part of an antitumor regimen in the treatment of ACP.

Keywords: Adamantinomatous Craniopharyngioma, Blood Brain Barrier, Tocilizumab

\section{FL-083}

\section{Neurotrauma/Critical Care}

Nakamura type I acute subdural hematoma in infant: Re-evaluation and contemporary role in diagnosis of AHT

Young Soo Park ${ }^{1}$, Nobuhiko Aoki ${ }^{2}$, Hiroshi Nishimoto ${ }^{3}$, Tamotsu Miki ${ }^{4}$, Masahiro Nonaka ${ }^{5}$, Mihoko Kato ${ }^{6}$, Nobuhito Morota $^{7}$

${ }^{1}$ Department of Neurosurgery and Children's Medical Center, Nara Medical University

${ }^{2}$ Department of Neurosurgery, Tokyo Metropolitan Tama Medical Center

${ }^{3}$ Division of Neurosurgery, Takenotsuka Noushinkei Rehabilitation Hospital

${ }^{4}$ Department of Neurosurgery, Tokyo Medical University

${ }^{5}$ Department of Neurosurgery, Kansai Medical University

${ }^{6}$ Department of Neurosurgery, Aichi Children's Health and Medical Center

${ }^{7}$ Division of Pediatric Neurosurgery, Kitasato University School of Medicine

OBJECTIVE: In Japan, acute subdural hematoma (ASDH) in infants has long been known to be caused by mild trauma at home, and is called Nakamura Type I. On the other hand, in Western countries, if there are three signs of subdural hematoma, retinal hemorrhage, and cerebral edema (= trias), shaken baby syndrome (SBS) immediately abusive head trauma (AHT) should be considered. This discrepancy has caused great confusion in diagnosis of AHT. This paper will evaluate and discuss the contemporary role of Nakamura Type I. 
MATERIAL-METHODS: Infantile ASDHs were accumulated from multiple centers in Japan and papers published from Japan were reviewed. The authors evaluated injured mechanism, neuroimaging findings, retinal hemorrhage and outcome in accidental ASDHs and compared with abusive ASDHs.

RESULTS: As already reported in the 1960s, the features of Nakamura type I ASDH were as follows: 1) Most affected infants were concentrated at 8-10 months of age. 2) Most of the injured mechanism were falls down backwards from stand posture holding on to things and almost all infants got a bruise on the occipital. 3) Subdural hematoma mainly located in interhemispheric fissure and bridging vein was damaged. Hematoma was caused by damage to bridging vein and associated cerebral contusion was extremely rare. 4) Retinal hemorrhage occurred in 20-60\%, but did not cause severe visual impairment. 5) Outcomes were good in $90 \%$, but $10 \%$ were more severe. Poor outcome was related to delayed surgical treatment and status convulsion.

On the other hand, in abusive ASDH, the majority had poor outcomes, secondary widespread brain damage due to respiratory disorders was a decisive factor and delays in hospital transport were also crucial issues.

CONCLUSION: It is extremely inappropriate to conclude as AHT solely due to the presence of the trias, and the possibility of Nakamura Type I should be considered especially in judgment by abuse experts.

Keywords: Nakamura Type I, infantile ASDH, SBS, AHT, judgment

\section{FL-084}

\section{Neurotrauma/Critical Care}

Decompressive craniectomies in children during the years 2009 - 2019 in Finland

Niina Salokorpi ${ }^{1}$, Tommi Korhonen ${ }^{1}$, Minna Oinas ${ }^{2}$, Atte Karppinen $^{2}$, Susanna Koponen ${ }^{3}$, Antti Lindgren ${ }^{3}$, Henna Henttonen $^{4}$, Minna Rauhala ${ }^{4}$, Anna Kotkansalo ${ }^{5}$

${ }^{1}$ Department of Neurosurgery, Oulu University Hospital, Oulu, Finland

${ }^{2}$ Department of Neurosurgery, Helsinki University Hospital, Helsinki, Finland

${ }^{3}$ Department of Neurosurgery, Kuopio University Hospital, Kuopio, Finland

${ }^{4}$ Department of Neurosurgery, Tampere University Hospital, Tampere, Finland

${ }^{5}$ Department of Neurosurgery, Turku University Hospital, Turku, Finland

OBJECTIVE: Decompressive craniectomy (DC) is an effective treatment of acute intracranial hypertension following traumatic brain injury (TBI) or stroke. No previous data on DC in pediatric population in Finland exists.

MATERIAL-METHODS: We evaluated all patients aged $\leq 16$ years who underwent DC during 2009-2019 in all five Finnish University Hospitals, where all acute neurosurgical cases are treated in Finland. Average follow-up time was 4 years.

RESULTS: In total, 83 craniectomies were done. Mean age at operation was 13 years (range 1 to 16 years), 59(71\%) were male. Twenty-four patients (29\%) died, most of whom within one week after DC and only one 3 years later. The primary insult was TBI in 61 cases, stroke in 8 , intracerebral haemorrhage in 9, encephalitis in 3 and diabetic ketoacidosis in one. One-sided craniectomy was perfomed for 63 patients, bifrontal for 11, bilateral for 8 and occipital for one. Cranioplasty was performed in 59 patients, in average 3 months after the DC. Autologous bone was used in 40 cases, and custom-made implants in the rest. Eleven cases required re-cranioplasty, 5 due to autologous bone resorption, and 5 due to infection. In 37 cases outcome was good or very good, with children able to continue normal education. 11 had moderate cognitive impairments and were dependent on helpgivers in daily life, and only one was in vegetative state. CONCLUSION: In this nationwide study, TBI was a main cause of acute intracranial hypertension in pediatric patients requiring DC. One-third of children who underwent DC died with majority of deaths occurring within first $48 \mathrm{~h}$. Surviving patients had a high probability of good recovery (63\%). Autologous bone resorption in our population was only $12.5 \%$.

Keywords: decompressive craniectomy, traumatic brain injury, cranioplasty, autologous, bone resorption

\section{FL-085}

\section{Neurotrauma/Critical Care}

Risk Factors of neurological deterioration in Pediatric Traumatic Epidural Hematoma

\section{Yi Zhang, Wei Shi, Rui Zhao, Hao Li}

Department of Neurosurgery, Children's Hospital of Fudan University, ShangHai, China

OBJECTIVE: To identify clinical factors associated with the neurological deterioration of traumatic epidural hematoma in children, provide evidence for early clinical intervention in potentially worsening cases.

MATERIAL-METHODS: A total of 203 hospitalized cases of "epidural hematoma" in our hospital in the past 5 years were Retrospective reviewed, and 168 cases were finally enrolled in the study. According to the Exclusion criteria, the patients were divided into stable group and exacerbation 
group, of which 104 cases were stable and 64 cases were exacerbation group. Presenting clinical and radiographic features were compared to find the significant differences relevant factors between the two groups.

RESULTS: GCS scores were similar at the time of treatment (14 vs 15$)$, while of which $38.1 \%(64 / 168)$ showed aggravating symptoms. The group exacerbation was more likely to present with altered mental status (34\% VS $15.4 \%$ $\mathrm{P}=0.004)$, temporal hematoma and mass effect (56.2\% VS $32 \% \mathrm{P}=0.012,53.1 \% \mathrm{VS} 13.5 \% \mathrm{P}=0.043$ ), lower average $\mathrm{CT}$ value hematoma $(48.4 \pm 6.77 \mathrm{VS} 60.7 \pm 5.25 \mathrm{P}=0.043)$, and increased D-dimer level $(42.2 \%$ VS $20.2 \% \mathrm{P}<0.01)$. Lower average $\mathrm{CT}$ value (OR: $13.1 ; 95 \% \mathrm{CI}: 0.5-1.8, \mathrm{P}<0.001)$ and mass effect(OR: 4.37 ; 95\% CI: 1.1-14.6,P $<0.05$ ) were predictive of poor clinical outcome in multivariate analysis. CONCLUSION: Traumatic epidural hematoma in children could be aggravated, clinical consciousness status and imaging examinations were useful for determining disease changes. The first CT Hu value and the occupancy effect were predictive of poor clinical outcome.

Keywords: Traumatic brain injury; epidural hematoma; neurological deterioration; conservative treatment.

\section{FL-086}

\section{Neurotrauma/Critical Care}

Mass lesion evacuation in the young presenting comatose carries similar outcome over last 2 decades: single institution series of 256 cases

Leon Levi ${ }^{1}$, Mony Benifla ${ }^{3}$, Josef Benari ${ }^{2}$, Vladimir Shapira $^{3}$, Iddo Paldor ${ }^{1}$, Marius Constantinescu ${ }^{1}$, Gill Sviri ${ }^{1}$ ${ }^{1}$ Neurosurgery department, Rambam healthcare campus, Haifa, Israel

${ }^{2}$ Pediatric intensive care department, Rambam healthcare campus, Haifa, Israel

${ }^{3}$ Pediatric neurosurgery unit, Rambam healthcare campus, Haifa, Israel

OBJECTIVE: Active neurotrauma registries may identify areas for needed improvement. To lessen the variability, we can use simple casemix benchmark either to reassess individual cases that are outliers of the prediction or changes over time period. The simple to use IMPACT-core + CT methodology was added into previous cohort but as it was validated on age 14 and up we added age subgroup of 14-18 to the traditional pediatric series. Severe brain injury $(\mathrm{GCS}<9, \mathrm{SHI})$ verified in neurotrauma and in need of mass evacuation is more homogenous group than relying on prehospital description. We will show the major changes in patterns of management and outcome in a single institute.
MATERIAL-METHODS: Records of consecutive acute admissions after SHI, age 0-18 during 1999-2018 and neurosurgery for mass lesion evacuation were matched with acute management. 6-month outcome and additional neurosurgery and rehabilitation needs were assessed.

RESULTS: There were 73 age $0-3,108$ age $4-13$ and 75 up to 17.99. Craniotomy was used to evacuate mass without midline shift in $73 \%$ of babies (vs $52 \%, \mathrm{p}=0.0086$ ). 111 patients had midline shift and 57 had decompressive craniectomy on the first operation while 26 of the 54 had to come back for secondary DC. There was significant change in mortality among age groups and periods between first CT with midline shift and absence of this $(28.6 \%$ vs. $2.8 \%$ mortality, $\mathrm{p}<0.0001)$. Resource utilization as hospital days and number of procedures was significantly higher in patients with shift ( $31 \pm 27$ vs $11 \pm 11, p=0.006) .63$ survivors of DC underwent cranioplasty, $21 \%$ needed another reconstructive procedure higher as one-half.

CONCLUSION: The series shows questionable outcome benefit of DC in children with midline shift with regard to survival and need for additional resources. The active registry can be used to highlight areas of further need and to validate the IMPACT benchmark to pediatric SHI.

Keywords: Severe head injur. prognosis. trauma registry

\section{FL-087}

\section{Neurotrauma/Critical Care \\ Protocol for Nonsurgical Management of Epidural Hae- matoma in Children: Experience in 153 Cases}

\author{
Artur Henrique Galvao Bruno Da Cunha \\ Department of Neurosurgery, Hospital da Restauração, \\ Recife, Brazil
}

OBJECTIVE: The traumatic brain injury (TBI) remains one of the main cause of morbidity and mortality in children. Tomographic images (CCT) of intracranial lesions, such as the presence of epidural haematomas (EDH), have guided surgical approaches even in asymptomatic or oligosymptomatic patients. The present series of cases intend to propose a protocol for the management of children with EDH, where the clinical criteria are as important as the CCT findings in the decision making.

MATERIAL-METHODS: Between March/2018 and March/2019, 153 patients, $72 \%$ male, aged 0-14 years, with a history of head trauma and a tomographic diagnosis of EDH were included in a conservative treatment protocol. Regarding the location of the EDH: frontal or frontal / parietal (66\%), temporal or temporal / parietal (29\%) and posterior fossa (5\%). The epidural hematoma volume ranged 
from 5 to 80 cc $(5-10$ cc $22 \%$; > 10- 15 cc $24 \%$; > 15-30 cc $29 \%$; > 30-60 cc 15\%; > 60 cc 11\%). All patients arrived with a normal pattern of consciousness and without focal neurological signs. $65 \%$ of patients were asymptomatic, while the rest had mild symptoms. The period between trauma and the emergency care ranged from $1 \mathrm{~h}-15$ days. They were kept under close observation for $6-48 \mathrm{~h}$.

RESULTS: Only two patients required craniotomy due to persistent headache and vomiting. The other patients were referred for outpatient follow-up asymptomatic, without neurological deficit.

CONCLUSION: Conservative treatment of EDH can be indicated in patients with ECG 15, asymptomatic or oligosymptomatic, without focal neurological signs, EDH volumes below $60 \mathrm{cc}$, midline deviation $<5 \mathrm{~cm}$ and with more than $6 \mathrm{~h}$ of trauma. We recommend strict observation for a period of 6 to $48 \mathrm{~h}$. This protocol can safely avoid unnecessary surgery.

Keywords: trauma, epidural hematoma, non-surgical approach, children

\section{FL-088}

\section{Neurotrauma/Critical Care Evaluation of the neurosurgical care of paediatric linear skull fractures}

Tom James Grundy, Roberto Ramirez, Ian Kamaly Asl, William John Kitchen

Department of Neurosurgery, Royal Manchester Children's Hospital, Manchester, United Kingdom

OBJECTIVE: Paediatric head injury is a common presentation. Very few of these cases will feature significant intracranial pathology which may necessitate neurosurgical intervention. Considerations regarding the duration, type and location of conservative management in paediatric head injury are of relevance and considered in this retrospective single centre observational study.

MATERIAL-METHODS: Review of the discharge and referral database of a large neurosurgical / major trauma regional This is a review of a prospectively maintained database at a tertiary paediatric neurosurgical centre. Cases of simple linear skull fracture and those with associated extraaxial haematoma were identified. Each patient's case at time of referral and been reviewed by a senior neurosurgeon and deemed to be managed non-operatively.

Data collected included age, GCS at initial assessment and neurological status. Length of hospital stay, safeguarding investigations, any repeat imaging and documented neurosurgical intervention $>12 \mathrm{~h}$ from referral were recorded.
Exclusion criteria included; age $>18$, depressed or comminuted skull fractures, polytrauma, intracranial haemorrhage not directly associated with linear skull fracture, cervical trauma and cases undergoing operative neurosurgical intervention within $12 \mathrm{~h}$ of referral.

RESULTS: A total of 426 cases of cranial trauma were reviewed. 196 cases of simple linear fracture and 62 cases of linear fracture associated with an extra-axial haematoma were identified. Initial GCS was 15 in $95.9 \%$ and $85.5 \%$ of cases respectively. Average LOS was 2.69 and 3.50 days.

1 case required repeat imaging due to clinical deterioration (seizure), 0 cases required neurosurgical intervention during inpatient observation or at 6 week review.

CONCLUSION: Children presenting with simple linear fractures and those with an associated non-surgical haematoma are unlikely to deteriorate. The majority of our cases where observed in a neurosurgical centre. We would suggest that these children could be observed in their referring hospital or discharged directly home providing a senior clinician with safeguarding training has reviewed the case.

Keywords: Linear Skull Fractures, Skull Fracture, Paediatric Head Injury, Neurosurgery Consultation, Head injury

\section{FL-089}

\section{Neurotrauma/Critical Care Metabolic and microstructural changes in children with severe TBI and HIBI. DTI and 1H MRS study}

Maxim Ublinskiy ${ }^{1}$, Andrei Manzhurtsev ${ }^{1}$, Tolibdzhon $\underline{\text { Akhadov }}^{1}$, Natalia Semenova $^{2}$, Alexei Yakovlev ${ }^{3}$

${ }^{1}$ Department of Radiology, Clinical and Research Institute of Emergency Pediatric Surgery and Trauma (CRIEPST), Moscow, Russian Federation

${ }^{2}$ Institute of Biochemical Physics, Russian Academy of Sciences, Moscow, Russian Federation

${ }^{3}$ Lomonosov Moscow State University, Moscow, Russian Federation

BACKGROUND AND AIM:Aim of this study was to explore the dynamics of microstructure and brain metabolism parameters in children with severe traumatic brain injury (sTBI) and hypoxic-ischemic brain injury (HIBI).

METHODS: 8 patients (mean age $=12.5$ ) with sTBI comprised group 1.4 children (mean age $=13.6$ ) with HIBI caused by drowning in fresh water. MR studies of patients from both groups were carried out twice: first—during the first seven days after injury (period 1); second-a month after injury (period 2). All studies were performed at Phillips Achieva 3.0 T MRI scanner. DT MR images were acquired with diffusion gradients applied in 32 non-collinear 
directions. 1H-MRS voxel (TR/TE $=1500 \mathrm{~ms} / 40 \mathrm{~ms}$, $\mathrm{NSA}=128$ ) was localized in left and right thalamus (for groups 1 and 2) and in brain stem (for group 2).

RESULTS: 1H MRS analysis in thalamus revealed significant decrease in dynamics of NAA/Cho value in group 1 (41\% decrease) and absence in dynamics of this index in group 2 (see Fig. 1). Significant increases in dynamics of $\mathrm{ADC}$ and FA values were found in corpus callosum in group 1 (see Fig. 2). In group 2 we detected increase in dynamics of ADC value and NAA/Cho ratio in brain stem (see Fig. 3). CONCLUSIONS:The significant decrease of NAA/Cho dynamics in thalamus in group 1 may indicate an active NAA intake in synthesis of oligodendrocytes to restore myelin sheath. Dynamics of spectroscopy and DTI parameters in brain stem correlates with the restoration of CNS functions in patients after drowning.

Keywords: traumatic brain injury, HIBI, MRI, MRS

\section{FL-090}

\section{Neurotrauma/Critical Care \\ Effects of hypertonic saline on ICP and cerebral autoreg- ulation in pediatric traumatic brain injury}

Julian Zipfel ${ }^{1}$, Juliane Engel ${ }^{2}$, Konstantin Ludwig Hockel ${ }^{3}$, Ellen Heimberg ${ }^{2}$, Feix Neunhoefer ${ }^{2}$, Martin Ulrich Schuhmann ${ }^{1}$

${ }^{1}$ Department of Neurosurgery, Division of Pediatric Neurosurgery, University of Tuebingen, Tuebingen, Germany ${ }^{2}$ Pediatric Intensive Care Unit, University Children's Hospital of Tuebingen, Tuebingen, Germany

${ }^{3}$ Department of Spine Surgery, Isar Klinikum, Munich, Germany

BACKGROUND AND AIM:Hypertonic saline (HTS) is commonly used in children to lower intracranial pressure (ICP) after severe traumatic brain injury (sTBI). While ICP and CPP correlate moderately to outcome in TBI, cerebrovascular autoregulation (AR) correlates well to neurological outcome. This study investigates the effect of HTS administration on ICP, CPP and AR in pediatric patients with sTBI. METHODS:28 intubated and sedated pediatric patients with STBI were included. Blood pressure and ICP were actively managed according to the autoregulation index PRx to determine and maintain an optimal CPP (CPPopt). In case ICP was continuously $>15-20 \mathrm{mmHg}$ (spending on age) despite all other measure to decrease ICP, an infusion of $3 \%$ HTS was administered. The monitoring data of the first $6 \mathrm{~h}$ after HTS administration were analyzed. GOS at 6 months was used as primary outcome measure and patients dichotomized in favorable (GOS 4-5) or unfavorable (GOS 1-3) groups.
RESULTS: Mean dose of HTS was $40 \mathrm{ml} 3 \% \mathrm{NaCl}$. No significant difference in ICP and PRx was seen between outcome groups after HTS administration. ICP was lowered significantly in all children, the effect lasting as long as $6 \mathrm{~h}$. Drop of ICP was significantly greater and longer in children with favorable outcome $(\mathrm{p}<0.001)$. Only this group showed a significant improvement of their autoregulatory capacity after HTS application $(p=0.048)$. A newly established HTS response index clearly separated outcome groups.

CONCLUSIONS:HTS significantly lowered ICP in all children after sTBI. This effect was significantly larger and longer lasting in children with favorable outcome. HTS administration restored disturbed AR only in the favorable outcome group. This highlights the role of a "rescuable" AR capacity regarding a better outcome, which might be a possible indicator of injury severity. The effect of HTS on autoregulation and possible mechanisms of its restoration after HTS application should be further investigated.

Keywords: traumatic brain injury, cerebrovascular autoregulation, hypotonic saline, injury severity, outcome

\section{FL-091}

\section{Occult tethered cord \\ Tethered cord diagnosis by MRI in supine and prone children positioning}

Cordula Scherer $^{1}$, Sonja Vulcu ${ }^{2}$, Nedelina Slavova ${ }^{3}$

${ }^{1}$ Department of pediatric surgery, University hospital Inselspital, Bern, Switzerland

${ }^{2}$ Department of neurosurgery, University hospital Inselspital, Bern, Switzerland

${ }^{3}$ Department of radiology, University hospital Inselspital, Bern, Switzerland

OBJECTIVE: Diagnosis of tethered cord in children is a combination of clinical signs and radiological results. In cases of typical findings, e.g. sacral porus, sacral hemangioma, neurogenic bladder or neurological deficits in the lower extremities, a MRI is indicated. Sometimes the results are ambiguous. To improve the diagnostic accuracy, for the last two years at our institution we perform diagnostic MRI in supine and prone position.

MATERIAL-METHODS: This was a retrospective analysis of our data from the years 2018 and 2019. MRI was done in the supine position and then in the prone position on a 1.5- or 3-T MR scanner. The patients were awake or under sedation/anesthesia. The average examination time including position change was $40 \mathrm{~min}$; mean patients age was 7.4 years. 
RESULTS: In our cohort $(n=27)$, imaging signs of tethered cord with a reduced ventral conus motion in prone position were found in $44.4 \%$ ( 12 cases). Of them, the majority ( $75 \%$ ) had a normal conus tip position above the L2/3 intervertebral disc level, and signs of tethering were detected only by using prone MRI.

CONCLUSION: In the assessment of suspected tethered cord in children, supine and prone MRI should be done when possible. In our experience, the combination of supine and prone MR is superior in the detection of tethered cord to supine position alone.

Our patients are followed up clinically with neurological and urodynamic examinations once a year until they are transitioned to adult-oriented health care at the age of 16. In our opinion surgery is only indicated if neurological deficits are present and worsening of the symptoms develops.

Keywords: tethered cord, prone positioning MRI

\section{FL-092}

\section{Occult tethered cord}

\section{Treatment strategy of complex meningocele}

\section{Kongbin Yang, Chunxu Li}

Neurosurgery department, 1st affiliated hosptal of Harbin University, Harbin, China

OBJECTIVE: To summarize and analyze the pathogenesis and treatment experience of complex meningocele.

MATERIAL-METHODS: From January 2009 to June 2016, 95 children with meningocele in neurosurgery department of the First Affiliated Hospital of Harbin Medical University were analyzed retrospectively. 15 children with meningocele were classified as complicated. The degree of loosening the tethered tissue was evaluated by Kirollos score, the nerve adhesion was relieved, and the arachnoid repair was used to prevent adhesion. All patients were followed up for 12 months.

RESULTS: Giant meningocele $(>10 \mathrm{~cm})$, meningocele with teratoma, lipoma (transitional) meningocele, recurrent meningocele and meningocele with giant lipoma were classified as complex meningocele in 4 cases, 2 cases, 2 cases, 3 cases and 4 cases respectively. According to Kirollos score evaluation during the operation, $61.6 \%$ of grade I, $15.2 \%$ of grade II and $15.2 \%$ of grade III were resulted. The operative effect rate was $73.3 \%$ apparently and $26.7 \%$ effectively. 4 cases of hydrocephalus, 2 cases of pseudomeningocele, 1 case of cerebrospinal fluid leakage and 1 case of incision infection were found during follow-up.

CONCLUSION: The technique of body position design, layered suture and sealing, combined cutting of internal and external terminal filament, releasing the adhesion nerves, and arachnoid repair can achieve good surgical results. There are many complications after the operation of complex meningocele, which need regular monitoring and follow-up.

Keywords: pediatric; complex meningocele; operation

\section{FL-093}

\section{Occult tethered cord \\ Split Cord and Concurrent Tethered Cord Syndrome: Case Series with Long-term Follow-up}

Andrew Joshua Kobets ${ }^{1}$, Adam Ammar ${ }^{2}$, Rick Abbott ${ }^{2}$, Alan R. Cohen ${ }^{1}$, Mari Groves ${ }^{1}$

${ }^{1}$ Department of Neurosurgery, Division of Pediatric Neurosurgery, Johns Hopkins Hospital, Baltimore, MD

${ }^{2}$ Department of Neurosurgery, Division of Pediatric Neurosurgery, Montefiore Medical Center, Albert Einstein College of Medicine, Bronx, NY

OBJECTIVE: The description of the natural course of concurrent tethered cord syndrome and diastematomyelia is lacking in the literature. We report the largest single cohort of patients with concurrent malformations and extensive long-term follow-up, with a focus on longitudinal management strategies.

MATERIAL-METHODS: Patients with concurrent diagnoses of diastematomyelia and tethered cord syndrome (radiographic evidence supporting clinical symptomatology) were identified over the period of 2000-2020, yielding 9 patients. On average, follow-up data was available for 5 years after the last surgery, and total follow-up since first surgery was 7.5 years (range $2-31$ years).

RESULTS: Nearly $78 \%$ of the cohort was female. The most common comorbidities included scoliosis, dermal sinus tracts, lipomas, and a Chiari I malformation. The most common symptoms were radiating leg pain and lower extremity paresthesias occurring in $44 \%$ of patients, with bladder/ bowel dysfunction, worsening scoliosis, and acute motor deterioration less common. Two patients were successfully treated conservatively for mild leg pain and paresthesias. For those who underwent surgery, all experienced alleviation of their symptoms upon first follow-up, although two had late recurrence of symptoms, one at 4 and 8 years, and the other at 11,26 , and 31 years after initial surgery. Both experienced years of improvement after each subsequent procedure.

CONCLUSION: The overlapping diagnoses of diastematomyelia and tethered cord, due to their rarity in the literature make management strategies difficult to formulate. Therefore, this series substantially supplements our knowledge of these patients, with on average nearly 8 years follow-up. Approximately $25 \%$ of patients were managed conservatively and saw an improvement in symptoms, confirming the 
practice of treating only those patients at risk of permanent neurological damage or intractable symptoms. Even with years of relief after surgical detethering, patients' symptoms may still recur over a decade after intervention, yet re-operation may still yield years of relief and should be considered.

Keywords: Diastematomyelia, split cord, tethered cord, lipoma, low-lying conus, fatty filum

\section{FL-094}

\section{Occult tethered cord}

The Double Layer Plastic of the Spinal Cord in Patients with Congenital Lumbar Hernia

\section{Abdurakhmon M Mamadaliev, Mansur A Aliev}

Neurosurgery Department, Samarkand State Medical Institute, Samarkand, Uzbekistan

OBJECTIVE: The success of the surgical treatment of the congenital lumbar hernias depends on the reliability of the performed plastic of posterior wall of the spinal cord. However, for the present time there are not the most reliable methods of plastic of posterior wall of the spinal cord.

MATERIAL-METHODS: We were observed 98 patients with congenital lumbar hernias at the age from 2 days to 12 years old who had hospital treatment in Neurosurgery Department of Samarkand Stae Medical Iinstitute for the last 12 years. Clinical investigation revealed that rough neurological disorders such as paresis and paralysis of lower extremity with disorders of the function of pelvic organs and sensational disorders were observed in $38,7 \%$ (38) of patients, partial neurological disorders (only lower paraparesis or disorders of the function of pelvic organs) were observed in 33,6\% (33) of patients and in 27,6\% (27) of patients we were not observed any neurological disorders. RESULTS: From 83 surgically operated patients in 42 (I group) it was performed the wide spreading method of plastic of posterior wall of the spinal cord by muscle-fascia approach and in 41 patients (II group) surgical operation was performed by using the new method - double-layer plastic of posterior wall of the spinal cord which was performed by use the following technique - the cutting out flaps from muscleaponeurosis tissue from both sides turn to the 180 degree and sewed by turns tightly and hermetically one by one.

CONCLUSION: In conclusion we consider that double-layer plastic of posterior wall of the spinal cord by use muscleaponeurosis flaps is the most effective method which could promote to decrease the different types of postoperative complications in patients with congenital lumbar hernias.

Keywords: Congenital, lumbar hernia, double-layer

\section{FL-095}

\section{Occult tethered cord \\ Surgery for the tethered cord syndrome following repair of lumbosacral congenital anomalies}

Nobuhito Morota

Department of Neurosurgery, Kitasato University Hospital, Sagamihara, Japan

BACKGROUND AND AIM:Management, surgical indication and surgical techniques for the postoperative tethered cord syndrome (TCS) following repair of lumbosacral congenital anomalies remain controversial.

METHODS:Sixty-nine patients underwent 79 surgeries for untethering the spinal cord following the initial repair surgery between 2002 and February 2021. Clinical data and factors leading to successful surgical management were retrospectively analyzed.

RESULTS: Original anomaly was $21 \mathrm{MMCs}, 43$ spinal lipomas, and 5 others. Duration from the initial repair to first untethering surgery ranged from 1 to 24 years (median 8 years). The rate of TCS by the presenting author was $24.6 \%$ (15/61) in MMC, and 13.3\% (26/195) in the conus spinal lipoma. Most common symptom was sensory-motor deterioration followed by worsened neurogenic bladder (NGB). Severe adhesion led to incomplete untethering in 3 patients (4.3\%) who had initial surgery at other hospitals and early cases in the series. Ten patients needed the second untethering surgery 2 to 10 years (median 7 years) after the first untethering. All surgery was performed under intraoperative neurophysiological monitoring (IONM). Postoperative complication was confirmed in 6 surgeries including 1 motor deterioration, 2 transit sensory disturbance and $4 \mathrm{CSF}$ leakage / pseudo meningocele in 6 surgeries (7.6\%). Majority of complications developed in patients with the occult spina bifida (OSB).

CONCLUSIONS:Long-term follow up is indispensable for patients who underwent surgery for the lumbosacaral congenital anomalies. Adult onset of TCS is not uncommon. Surgery is often challenging, especially in case of spinal lipomas and other types of OSB. Re-tethering after the first untethering is not unusual and preventive technique is required in the first untethering surgery. IONM plays critical role in preventing postoperative neurological complications in surgery of TCS.

Keywords: Tethered cord syndrome, surgery, untethering, myelomeningocele, spinal lipoma, occult spina bifida

\section{FL-096}




\section{Occult tethered cord \\ Occult tethered cord syndrome: a rare treatable condition}

Jeyul Yang ${ }^{1}$, Jae Kyung Won ${ }^{2}$, Kyung Hyun $\mathrm{Kim}^{3}$, Ji Yeoun $\mathrm{Lee}^{4}$, Ji Hoon $\mathrm{Phi}^{3}$, Seung Ki Kim ${ }^{3}$, Kwanjin Park ${ }^{5}$, Kyu Chang Wang ${ }^{1}$

${ }^{1}$ Neuro-oncology clinic, National Cancer Center, Goyang, Korea

${ }^{2}$ Department of Pathology, Seoul National University Hospital, Seoul, Korea

${ }^{3}$ Division of Pediatric Neurosurgery, Seoul National University Children's Hospital, Seoul, Korea

${ }^{4}$ Division of Pediatric Neurosurgery, Seoul National University Children's Hospital, Seoul, Korea; Department of Anatomy, Seoul National University College of Medicine, Seoul, Korea

${ }^{5}$ Division of Pediatric Urology, Seoul National University Children's Hospital, Seoul, Korea

BACKGROUND AND AIM:Occult tethered cord syndrome (OTCS) is a controversial entity that involves urinary dysfunction in patients with a normal spinal MRI. The purpose of this study was to evaluate the existence, prevalence, histological characteristics and surgical outcomes of OTCS.

METHODS:We retrospectively analyzed patients who underwent untethering surgery for OTCS from January 2010 to December 2019. OTCS was defined as (1) clinical manifestation of tethered cord, (2) symptoms supported by urodynamic study (UDS) or electromyography/nerve conduction study, (3) a conus medullaris located in normal position, and (4) a normal thickness filum terminale $(<2 \mathrm{~mm})$. Patients with structural lesions in the urological tract, developmental delay, syringomyelia, caudal agenesis or other apparent anatomic findings of tethered cord were excluded. All patients underwent UDS preoperatively and post-void residual urine (PVR) was recorded. Sectioned fila of OTCS patients were histologically reviewed.

RESULTS: Five out of 439 patients (1.1\%) who underwent untethering surgeries for occult spinal dysraphism during the same period corresponded to OTCS. Patients had detrusor-sphincter dyssynergia (DSD) with wide range of PVRs. Histological finding of OTCS patients showed high percentage of fibrous tissue in fila as was revealed in TCS patients. There were two patients who showed improvement after untethering surgery. Two patients had no improvement nor aggravation, and one patient showed temporal improvement followed by re-aggravation. The patients who improved experienced sudden onset of urinary symptoms. Symptoms completely resolved in patients who experienced rapid progression.

CONCLUSIONS:We think that OTCS is a definitely existing entity, although extremely rare. The fila showed high percentage of fibrous tissue as in TCS patients. Sudden onset of urinary dysfunction or pain with rapid progression, along with DSD proven by UDS is the best indication for untethering surgery in OTCS patients. Postoperative urological care should be continuously taken in order to avoid re-aggravation.

Keywords: Occult tethered cord syndrome, spinal dysraphism, urodynamic study, detrusor-sphincter dyssynergia, unethering

\section{FL-097}

\section{Other}

Cerebrospinal fluid leakage after cranial surgery in the pediatric population - Preliminary results of a systematic review and meta-analysis

Emma M.H. Slot ${ }^{1}$, Kirsten M. van Baarsen ${ }^{2}$, Eelco W. Hoving $^{2}$, Peter Zuithoff ${ }^{3}$, Tristan P.C. van Doormaal ${ }^{4}$

${ }^{1}$ Department of Neurology and Neurosurgery, University Medical Center Utrecht, Utrecht, The Netherlands; Brain Technology Institute, Utrecht, The Netherlands

${ }^{2}$ Department of Neuro-oncology, Princess Máxima Center for Pediatric Oncology, Utrecht, The Netherlands

${ }^{3}$ Julius Center for Health Sciences and Primary Care, University Medical Center Utrecht, Utrecht, The Netherlands

${ }^{4}$ Department of Neurology and Neurosurgery, University Medical Center Utrecht, Utrecht, The Netherlands; Brain Technology Institute, Utrecht, The Netherlands; Department of Neurosurgery, University Hospital Zürich, Zürich, Switzerland

OBJECTIVE: Cerebrospinal fluid (CSF) leakage is one of the major complications after cranial neurosurgical interventions. It is associated with substantial morbidity and increased healthcare costs. The magnitude of the problem in children, however, is still unknown. This meta-analysis aims to quantify the rate of CSF leakage in the pediatric population in different subgroups based on surgical category. MATERIAL-METHODS: The authors followed the PRISMA guidelines. The Embase, PubMed and Cochrane databases were searched for studies reporting CSF leakage and related complications after intradural cranial surgery in patients $\leq 18$ years. Studies on transsphenoidal surgery or burr hole surgery (including primary CSF diversion surgeries) were excluded. Meta-analysis of proportions was performed using a generalized linear mixed model for binomial outcomes.

RESULTS: The database search yielded 2,060 articles, of which 26 were included. A total of 2,971 patients who underwent 3,076 surgeries were included. The overall CSF leakage rate was $4.8 \%$ (95\% CI 2.7 to $8.3 \%$ ). The highest 
CSF leakage rate was observed in patients undergoing craniectomy $12.5 \%$ (95\% CI 5.4-26.1\%), for craniotomy it was $2.5 \%$ (95\% CI $1.1-5.6 \%$ ). A CSF leakage rate of $7.2 \%$ (95\% CI \% 4.3-11.9\%) was found for infratentorial surgery in contrast to $1.1 \%$ (95\% CI \% $0.3-4.0 \%$ ) for supratentorial surgery. The percentage of CSF leakage in patients undergoing decompression for Chiari malformation was 7.5\% (95\% CI $2.9-18.0 \%$ ) and $7.7 \%$ (95\% CI 4.4-13.1\%) for posterior fossa tumor surgery. Further subgroup analyses, for example epilepsy, vascular and trauma subgroups, could not be performed due to insufficient data quality. The exact definition of CSF leakage was unclear in the majority of studies.

CONCLUSION: CSF leakage after cranial surgery is a significant problem in the pediatric population, especially in patients undergoing craniectomy and infratentorial surgery.

Keywords: CSF leakage, complications, craniotomy, craniectomy, fossa posterior tumor surgery, Chiari malformation

\section{FL-098}

\section{Other}

\section{Physician Influence on Parental Decision to Allow Child} Participation in Sports

Natalie Hibshman ${ }^{1}$, Aaron Yengo Kahn ${ }^{2}$, Alyssa Wiseman ${ }^{1}$, Patrick Kelly $^{2}$, Chevis Shannon ${ }^{1}$, Christopher Bonfield ${ }^{2}$

${ }^{1}$ Surgical Outcomes Center for Kids, Monroe Carell Jr. Children's Hospital at Vanderbilt, Nashville, TN, USA

${ }^{2}$ Department of Neurological Surgery, Vanderbilt University Medical Center, Nashville, TN, USA

OBJECTIVE: The decision to allow a child to participate in sports is multifaceted. Frequently, parents desire information from additional sources, such as physicians. Utilizing a national survey for parents, we sought to understand what factors lead to a parent seeking physician decision support. MATERIAL-METHODS: 916 parents completed a 31-question survey regarding child sport participation. Responses were recorded per household-child (1516 total). Sports were categorized into groups (collision, contact, limited contact, and extreme). Parent and child demographics, decision influences to allow or disallow participation, and concussion history were obtained.

RESULTS: Of 1516 children, 516 had parents who used physician input in their decision making. Male parents were less likely to cite physician influence. Parents citing physician influence were more likely to have a master's degree or higher, were more often in a dual decision-maker household, and identified safety as the primary concern to disallow a sport. Those who cited physician influence cited more influences overall such as spouse, other parents, and the media.
When deciding participation in collision and extreme sports, parents cited physician influence more often. Parents who do not cite physician influence are more likely to report child desire as the biggest influence in their decision. No parent or child demographic factors (including past concussion history) reliably predicted the use of physician influence in this decision. Parents who identify child's desire or the cost of the sport were less likely to utilize physician influence in making the decision.

CONCLUSION: Parents who reported using physiciangiven information to help determine their child's sport participation represent a diverse group of cautious people, incorporating many sources of information into this decision. Child desire to play a sport and cost of the sport are other strong influences on the decision. Better understanding which parents are influenced by physicians aids in clinical shared decision making and promotes effective communication.

Keywords: contact sports,collision sports, parental decision-making, physician influence

\section{FL-099}

\section{Other}

Treatment of Pediatric Chronic Headache by Epidural Saline and Oxygen Injection

Kiyoshi Takagi ${ }^{1}$, Kenji Onouchi ${ }^{2}$, Kazuyoshi Kato ${ }^{3}$, Takashi Kawahara $^{4}$, Masamichi Atsuchi ${ }^{4}$

${ }^{1}$ Department of Neurosurgery, Nagareyama Central Hospital, Nagareyama, Japan

${ }^{2}$ Department of Neurology, Tsukuba Hospital, Tsukuba, Japan

${ }^{3}$ Department of Surgery, Abiko-seijinkai Hospital, Abiko, Japan

${ }^{4}$ Department of Neurosurgery, Atsuchi Neurosurgical Hospital, Kagoshima, Japan

OBJECTIVE: We have reported that epidural saline and oxygen injection (ESOI) was safe and effective to treat adult chronic post traumatic headache (Acta Neurochir Suppl. 118; 293-296, 2013). There are many pediatric patients suffering from so severe headache to continue studying with or without preceding trauma. Since ESOI is safe and almost painless, the purpose of this study is to report the results of applying this treatment to the pediatric patients with severe chronic headache.

MATERIAL-METHODS: Thirty-six pediatric patients (less than 15 years old) were the candidates of this study. They had severe headache for over 3 months and previously received several kinds of conservative treatments with vain. Neuroimaging studies were normal. They had 
no definite neurological deficits. The treatment procedure was almost the same with sacral anesthesia. Sacral hiatus was punctured and $5 \mathrm{~mL}$ saline and filtered oxygen up to $80 \mathrm{~mL}$ was injected into epidural space. The outcome was evaluated 3 months after (Excellent: the patients returned to school life, Fair: headache diminished but not returned to school, No: no improvement of the headache, Poor: worsened symptoms).

RESULTS: Median age was 13 yo (IQR, 13-14). There were 16 boys (median age was 13 (IQR, 11.25-13.75)) and 20 girls (median age was 14 (IQR, 13-14)). Girls were significantly older than boys. Twenty patients had previous trauma but 16 patients had no history of trauma. There were no serious complications and we had no poor outcome. Twenty-five patients showed excellent (14 with trauma and 11without trauma) and 11 patients showed fair outcome.

CONCLUSION: Although the mechanism was not clear, ESOI was effective for the chronic with or without previous trauma. Epidural saline and oxygen injection can be an effective treatment for the pediatric severe chronic headache.

Keywords: chronic headache, chronic posttraumatic headache, treatment, epidural oxygen injection.

\section{FL-100}

\section{Other}

\section{Comparison of Aesthetic Outcomes between Open and} Endoscopically Treated Sagittal Craniosynostosis

Jacob Richard Lepard ${ }^{1}$, Hassan Akbari ${ }^{1}$, James Mooney ${ }^{1}$, Samuel Mccluggage ${ }^{1}$, Renee Meyers ${ }^{2}$, John Grant ${ }^{2}$, James M Johnston ${ }^{1}$

${ }^{1}$ Department of Neurological Surgery, University of Alabama at Birmingham, Birmingham, Alabama, USA

${ }^{2}$ Department of Plastic Surgery, University of Alabama at Birmingham, Birmingham, Alabama, USA

OBJECTIVE: Surgical treatment of pediatric craniosynostosis includes both open reconstruction and endoscopic treatment with cranial orthosis. Clinical outcomes and cost-effectiveness have been compared between the two techniques. The purpose of the current study is to assess aesthetic outcomes between open and endoscopically treated pediatric patients.

MATERIAL-METHODS: Fifty patients with single suture sagittal synostosis were recruited for inclusion. Standardized post-operative photographs were taken at follow-up clinic visit and assessed by faculty surgeons, trainee surgeons, nursing staff, and lay persons. Ratings were given based on normal or abnormal appearance using a 5-point Likert scale. Mann-Whitney U and multivariate linear regression were used to assess for differences between groups.
RESULTS: A total of 31 patients underwent open surgical treatment and 19 underwent endoscopic. $90 \%$ of patients were male (45/50). There was no difference between groups in male to female ratio. Z-score of post-operative head circumference normalized by age were not significantly different between groups $(\mathrm{p}=0.11)$. Median aesthetic rating for the open and endoscopic groups were 3.74 (IQR 3.4-4.3) and 4.32 (IQR 4-4.47), respectively. The aesthetic ratings were significantly higher for patients treated endoscopically compared with standard open $(\mathrm{p}=0.002$, and remained so when controlled for age, gender, and ethnicity $(p=0.026)$. Length of stay, transfusion volume, blood loss, and OR time were all significantly lower in the endoscopic group compared to open $(\mathrm{p}<0.001)$.

CONCLUSION: Despite higher overall aesthetic ratings for the endoscopic group compared with open, when controlled for age, gender, and ethnicity there was no statistically significant difference between groups. This is important information for pre-operative family counseling and surgical decision making.

Keywords: Craniosynostosis; Sagittal; Aesthetic; Endoscopic; Cranial Vault Reconstruction

\section{FL-101}

\section{Other}

Foramen Magnum Decompression surgery for Paediatric Chiari I malformation: an aetiology based approach

Alexandra Valetopoulou ${ }^{1}$, Maria Constantinides ${ }^{1}$, Adikarige Silva $^{2}$, Dominic Thompson ${ }^{2}$, Zubair Tahir ${ }^{2}$

${ }^{1}$ Barts and the London School of Medicine and Dentistry, London, United Kingdom

${ }^{2}$ Department of Neurosurgery, Great Ormond Street Hospital for Children, London, United Kingdom

OBJECTIVE: The published outcomes of foramen magnum decompression surgery for Chiari 1 malformation (CIM) in children are variable. The aim of this study was to evaluate the efficacy of an "aetiology based" modification to treatment

MATERIAL-METHODS: Children who underwent Foramen Magnum Decompression (FMD) for CIM in a single institution over 35-years (1985-2020) were reviewed.

RESULTS: 230 children, 35 were excluded (incomplete data) leaving 195 children (116 females, 79 males) in our review.

The commonest diagnosis was idiopathic tonsillar ectopia ('classical' CIM) (82\%). Additional diagnoses included raised ICP due to hydrocephalus (3.6\%), or craniosynostosis 
(8.2\%), cranio-vertebral junction (CVJ) malformation (5.6\%) and one child had dual pathology. Syringomyelia was present in 119 children (61\%). The commonest clinical presentations were suboccipital headaches (44\%) and motor dysfunction $(28.7 \%)$.

The operative strategy included: bone-only FMD (35.9\%), FMD with dural splitting (8.7\%) and FMD with duraplasty (55.4\%). Seven children with CVJ-malformation had decompression with occipito-cervical fixation. All children with hydrocephalus had CSF-diversion pre-FMD. Two children with craniocerebral disproportion had FMD pre vault expansion surgery.

Symptom resolution was observed in $82.4 \%$ of duraplasty cases, $74 \%$ of bone-only cases and $70.6 \%$ of dural splitting cases. Improvement in syrinx status was seen in $82.7 \%$ of duraplasty cases, in $37.5 \%$ of dural splitting cases and in $30.2 \%$ of bone only cases.

25 patients required revision FMD surgery (15/70 bone only FMD, 1/17 FMD with dural splitting and 9/108 FMD with duraplasty) owing to persisting/recurrent symptoms and/or syringomyelia, with 3 requiring a second revision. Patients requiring revision FMD sub-classified aetiologically included 17/160 idiopathic, 5/16 craniosynostosis, 2/7 hydrocephalus, 1/11 with CVJ malformation.

CONCLUSION: It is incumbent upon the neurosurgeon to identify the underlying pathophysiological mechanism that has led to the CIM to select the most appropriate surgical strategy. The appropriate operation should be carefully selected depending on the underlying pathology in order to optimise surgical outcomes.

Keywords: Chiari 1 malformation, Foramen magnum Decompression

\section{FL-102}

\section{Other}

Treatment of Pediatric Cerebral Venous Sinus Thromboses: the Role of Anticoagulation

Nathan A. Shlobin ${ }^{1}$, Melissa A. LoPresti ${ }^{2}$, Molly Beestrum ${ }^{3}$, Sandi K. Lam ${ }^{1}$

${ }^{1}$ Department of Neurological Surgery, Feinberg School of Medicine, Division of Pediatric Neurosurgery, Lurie Children's Hospital, Chicago, USA

${ }^{2}$ Department of Neurosurgery, Baylor College of Medicine, Division of Neurosurgery, Texas Children's Hospital, Houston, USA

${ }^{3}$ Department of Library Services, Northwestern University Feinberg School of Medicine, Chicago, USA

OBJECTIVE: Cerebral venous sinus thromboses occur in children for a variety of reasons. However, no standard treatment paradigm is established. We sought to identify what treatments have been applied, what their outcomes have been, and what the role of anticoagulation is in pediatric patients with CVST.

MATERIAL-METHODS: A systematic review was conducted exploring all treatments of pediatric CVSTs using the PubMed, Embase, Scopus, and Cochrane Library and Cochrane Central Register of Controlled Trials databases. Titles and abstracts from all articles identified were read and selected for full text review. Studies meeting inclusion criteria were reviewed in full and analyzed for: study design, aim, population, interventions, and outcomes.

RESULTS: Of 2,946 resultant articles, 51 full text articles were included for review. Management of infectious CVST included broad-spectrum antibiotics, surgery, and anticoagulation. Neoplastic and traumatic CVST treatment included anticoagulation. Treatment of CVSTs associated with metabolic abnormalities centered on correction of metabolic derangements, or supplementation where appropriate, and anticoagulation. Autoimmune, congenital, and thrombotic pathway CVSTs were treated with anticoagulation in addition to treatment of the underlying disorder. The most commonly used anticoagulants included unfractionated heparin and low molecular weight heparin; both were seen to be effective and safe. Uncommonly, endovascular interventions were used with varying success.

CONCLUSION: While conservative treatment with hydration is often applied as a first step, anticoagulation for treatment of CVST is reported to be safe and effective in pediatric patients. Risks and benefits of anticoagulation or thrombolytic therapy in these patients must be weighed carefully on a case by case basis as no randomized controlled trials have established a standard of care. We look forward to the development of guidelines regarding specific anticoagulation regimens, including dosage and duration, algorithms for reimaging during disease course, and integration of patient-specific considerations to weigh and stratify the risk of the treatment in pediatric CVST patients.

Keywords: pediatric neurosurgery, cerebral venous sinus thrombosis, deep venous thrombosis, sinus thrombus, anticoagulation, endovascular intervention

\section{FL-103}

\section{Other}

Sleep Disordered Breathing in Pediatric Patients with Chiari I Malformation

Gregory Albert ${ }^{1}$, Eylem Ocal ${ }^{1}$, Tomoko Tanaka ${ }^{1}$, James Hungerford $^{2}$, Gresham Richter ${ }^{3}$, Heather Wright ${ }^{3}$, Moira Moore $^{1}$, Supriya Jambhekar ${ }^{2}$ 
${ }^{1}$ Division of Pediatric Neurosurgery, Arkansas Children's Hospital, Little Rock, AR, USA; Department of Neurosurgery, University of Arkansas for Medical Sciences, Little Rock, AR, USA

${ }^{2}$ Division of Sleep Medicine, Arkansas Children's Hospital, Little Rock, AR, USA; Department of Pediatrics, University of Arkansas for Medical Sciences, Little Rock, AR, USA

${ }^{3}$ Division of Pediatric Otolaryngology, Arkansas Children's Hospital, Little Rock, AR, USA; Department of Otolaryngology, University of Arkansas for Medical Sciences, Little Rock, AR, USA

OBJECTIVE: Chiari I malformation (CM1) has a wellknown association with sleep apnea. However, few studies have examined this in detail in children with CM1. Prior studies have suggested that the incidence of sleep apnea may be underdiagnosed in this patient population. In addition, results of surgical decompression have not been evaluated. MATERIAL-METHODS: In May 2019, we instituted a new CM1 management protocol whereby all patients newly diagnosed with CM1 are evaluated with polysomnography (PSG) and undergo and upper airway evaluation in the otolaryngology clinic. We collected demographic data (age, gender), data on presenting symptoms, reported sleep symptoms, and clinical/radiographic evidence of dysphagia. Pediatric patients seen for new diagnosis CM1 in the neurosurgery clinic between May 2019 and April 2020 and had PSG were included. The local institutional review board deemed this study not human subjects research.

RESULTS: We have evaluated 18 patients to date with PSG, 11 of whom are female. The mean age is 8.3 years (range 0.8 - 16.6 years). Three patients have obstructive sleep apnea (OSA), 4 patients have central sleep apnea (CSA), and 1 patient has mixed sleep apnea. Ten patients had normal PSG. Age across groups was not different. All patients with sleep-disordered breathing reported symptoms prior to PSG, most often snoring. In addition, $8(80 \%)$ of the patients with normal PSG reported sleep symptoms. To date, 1 patient with OSA, 2 patients with CSA, and the patients with mixed apnea have had Chiari decompression. Surgery has been recommended in 1 additional OSA patient. Follow-up is ongoing.

CONCLUSION: Sleep disordered breathing is likely underrecognized in patients with CM1. These results support this conclusion. Data collection is ongoing, including evaluating more patients, collecting postoperative follow-up data, and determining predictors of sleep apnea and response to surgery in these patients.

Keywords: Chiari malformation, sleep disordered breathing, sleep apnea

\section{FL-104}

\section{Other}

Evolution of Surgical Management of Sagittal Synostosis - A Single Institution Review Short Title: Evolution of Sagittal Synostosis Management

$\underline{\text { Hasan Syed }}^{1}$, Mariam Ishaque ${ }^{1}$, Jon Black ${ }^{2}$, Kelly O'Connell ${ }^{1}$, John Jane, Jr ${ }^{1}$

${ }^{1}$ Department of Neurosurgery, University of Virginia, Charlottesville, VA, USA

${ }^{2}$ Department of Plastic Surgery, University of Virginia, Charlottesville, VA, USA

OBJECTIVE: Numerous methods for surgical correction of sagittal synostosis have been described in the literature, yielding similar outcomes. At our institution, surgical approaches to correct this condition have evolved over the past few decades, including $\Pi$, H-type craniectomies (Renier), endoscopic suturectomy, and our current technique, the FLAG procedure. Our aim is to review the evolution of these surgical techniques at our institution and compare patient outcomes.

MATERIAL-METHODS: A retrospective review was performed on consecutive patients undergoing correction for craniosynostosis from 2008 to 2018. All patients with a diagnosis of nonsyndromic isolated sagittal craniosynostosis were included and classified into one of 4 groups by the type of surgical correction performed (H-type, FLAG, endoscopic, other).

RESULTS: The authors identified 166 consecutive patients with a mean age at time of surgery of $6.7 \pm 4.0$ months. $91(54.8 \%)$ carried a diagnosis of nonsyndromic sagittal synostosis. 63 patients underwent $\mathrm{H}$-type procedures, 9 underwent FLAG procedures, 5 underwent endoscopic procedures, and 14 were classified as other (distraction or implant). Perioperatively, the FLAG group had the shortest ICU stay $(1.3$ days, $p<0.05)$, postoperative transfusion requirement (42 cc pRBC, $\mathrm{p}<0.001$ ), and complication rate $(0.0 \%)$. The endoscopic group had the shortest surgical time at $2.00 \mathrm{~h}(\mathrm{p}<0.001)$. No statistically significant difference in cranial index or revision procedures between the four groups was identified. Overall, the mean length of follow-up was 25.3 months.

CONCLUSION: All procedures had similar results for cranial index with decreased surgical time, transfusion volume, and hospital stay seen in FLAG and endoscopic groups.

Keywords: Craniosynostosis, Sagittal synostosis

FL-105

Other

A Novel Quantitative Method for Assessment of the surgical Outcome of Patients with Craniosynostosis 
Young Sill Kang ${ }^{1}$, Min Jin Lee ${ }^{3}$, Helen Hong ${ }^{3}$, Kyu Won Shim $^{2}$

${ }^{1}$ Department of Neurosurgery, University Medical Center Schleswig Holstein, Kiel, Germany

${ }^{2}$ Department of Pediatric Neurosurgery, Yonsei University College of Medicine, Severance Children's Hospital, Seoul, South Korea

${ }^{3}$ Department of Software Convergence, Seoul Women's University, Seoul, South Korea

OBJECTIVE: Several methods for the quantitative assessment of the surgical effects of craniosynostosis have been suggested. Currently, cephalic index is considered as the most common measure for evaluating the surgical effect of craniosynostosis, which has been shown limitations. We propose a quantification method for evaluating the surgical effects on regional cranial bones by comparing preoperative and postoperative skull shapes.

MATERIAL-METHODS: For this study, 78 patients with CTs were analysed. The mean ages of the subjects before and after surgery were 11.03 months (SD: 7.25 months, range: 2 days to 36 months) and 27.94 months (SD: 10.44 months, range: 12 to 60 months), respectively and the mean age interval before and after surgery was 17.16 months (SD: 7.64 months, range: 3 to 45 months). The methods consist of three main steps. (a) preprocessing skull surface and generation of mean normal skull model. (b) regional bone partitioning and performing shape deformation estimation, (c) quantification of skull shape deformation between preoperative and postoperative skulls.

RESULTS: In sagittal synostosis, the frontal and occipital bones showed a large reduction index, and the parietal bones showed a large expansion index. In case of bicoronal synostosis, the frontal and parietal bones demonstrated a large reduction index, and the parietal and occipital bones showed a large expansion index. In unilateral bicoronal synostosis with asymmetric shape, the right frontal and parietal bones showed a large reduction index, and the left frontal and parietal bones, and occipital bone showed a large expansion index. In case of unilateral lambdoid synostosis, the right frontal and partial bones showed a large reduction index, and the left frontal and parietal bones and occipital bone showed a large expansion index.

CONCLUSION: The proposed quantification method overcomes the limitations and provides useful information for quantifying the surgical effects of craniosynostosis patients with symmetric and asymmetric deformities.

Keywords: Quantification, Craniosynostosis, Regional cranial bone, Morphological changes, indices

\section{Other}

Isolated Frontosphenoidal Craniosynostosis: The Alder Hey Experience and a Novel Algorithm to Aid Diagnosis

Anusha Hennedige, Christopher Parks, Jonathan Ellenbogen, Ajay Sinha, Christian Duncan, David Richardson Alder Hey Children's Hospital, Department of Craniofacial Surgery, Liverpool, England

BACKGROUND AND AIM:Unilateral synostotic frontal plagiocephaly is most commonly due to a premature fusion of the frontoparietal suture. However, the coronal ring comprises of major and minor sutures and these sutures in isolation or in combination can result in similar clinical presentations which can make diagnosis challenging and result in a delay in referral to a craniofacial surgeon for timely management.

METHODS:A retrospective review of all patients with unicoronal synostosis were identified and those with isolated frontosphenoidal craniosynostosis was identified. Patients case notes and radiographs were reviewed. A literature review of previous case reports of frontosphenoidal craniosynostosis was performed.

RESULTS: We present our series of 4 patients to add to this cohort of patients and describe key characteristics to distinguish frontoparietal from isolated frontosphenoidal synostosis and introduce a means of differentiating these 2 diagnoses from posterior deformational plagiocephaly and unilateral lambdoid synostosis. Isolated frontosphenoidal craniosynostosis is a rare clinical entity with only 49 cases reported in the English literature to date.

CONCLUSIONS:Frontosphenoidal craniosynostosis is a rare presentation and often missed by clinicians hence late presentation is not unusual. All previous case reports have been diagnosed after radiological imaging but we have devised a novel algorithm to aid the clinician in diagnosis of craniosynostosis prior to any radiological imaging.

Keywords: Frontosphenoidal, Craniosynostosis, Frontoparietal

\section{FL-107}

\section{Other}

The level of $\boldsymbol{\gamma}$-aminobutyric acid in posterior cingulate cortex of children with mild TBI

Andrei Manzhurtsev ${ }^{1}$, Maxim Ublinskiy ${ }^{1}$, Alexey Yakovlev $^{2}$, Tolib Akhadov ${ }^{1}$, Natalia Semenova ${ }^{3}$

${ }^{1}$ Clinical and Research Institute of Emergency Pediatric Surgery and Trauma

\section{FL-106}


${ }^{2}$ Emanuel Institute of Biochemical Physics of the Russian Academy of Sciences

${ }^{3}$ Moscow State University

BACKGROUND AND AIM:The mechanisms underlying functional disorders in the acute concussion require intensive research. The imbalance between the major neurotransmitters, excitatory - glutamate, and inhibitory, GABA, may be crucial for CNS function after mild TBI (mTBI). In this study GABA is measured in the posterior cingulate cortex (PCC) of children with the acute mTBI.

METHODS:Twelve patients with acute mTBI (12-70 h since the injury, $15.7 \pm 1.9$ y.o.) and thirteen healthy subjects $(19.3 \pm 0.7$ y.o.) participated in the study. MRI scanner Philips Achieva 3.0 T was used, standard MRI protocol for TBI patients (T2-, T1-wighted images, FLAIR, SWI, DTI) revealed no pathological lesions in brain tissue of any subject. Magnetic resonance spectroscopy voxels were located in posterior cingulate cortex. MEGA-PRESS pulse sequence was used: $\mathrm{TR}=2000 \mathrm{~ms}$, $\mathrm{TE}=80 \mathrm{~ms}$, editing pulses on $1.9 \mathrm{ppm}$ and $1.5 \mathrm{ppm}$, time $10 \mathrm{~min}$. GABA spectra were processed in Gannet 3.0. Mann-Whitney criterion was used to reveal the significance cat between-group differences. RESULTS: The increase in GABA in patient group was $+12 \%, p<0.05$. The levels of other MRS-detectible metabolites demonstrated no between-group differences.

CONCLUSIONS:The result obtained in current work agrees with our previous study, where GABA was increased $(\mathrm{p}<0.005)$ in the anterior cingulate cortex $(\mathrm{ACC})$ of children with mTBI when comparing to healthy controls. This may signify the shift of the neurotransmitter balance towards inhibition. Further data collecting (e.g. in different regions) is required in order to find alterations in other cerebral loci.

Keywords: GABA, mTBI, posterior cingulate cortex

\section{FL-108}

\section{Spine}

From Pedicle Realignment to Pedicle Reformation for management of paediatric Hangmans fracture

Pankaj Kumar Singh, Dattaraj Sawarkar, Satish Verma, Sarat Chandra, Shashank Kale

Department of neurosurgery, All India in statute of medical sciences,New Delhi,India

OBJECTIVE: There is no literature available detailing the management of paediatric hangman's fracture. The aim of this study was to study outcomes in paediatric cases of hangman's fractures and to demonstrate evolution in management of hangman's fracture from anatomical realignment of $\mathrm{C} 2$ pedicle to pedicle reformation in old and complicated hangman's fracture.

MATERIAL-METHODS: This is a retrospective study. Nine paediatric patients operated at a tertiary level centre between. September 2011 to February 2018 were included. Preoperative and postoperative neurological assessment was done using the ASIA scoring. Details of type of fracture, operating time, blood loss, follow up and complications were assessed.

RESULTS: Patients' age ranged from 14 to 18 years (mean 16.45 years). Mean follow up was 42.78 (range 12-81) months. Three patients had no preoperative neurological deficit. Six patients havin neurological deficit showed improvement in ASIA score. Two patients, one having undergone. C1 C3 LMSF and other had C2 pars C3 LMSF, developed kyphosis for which fixation was.

further extended caudally. One patient with an old hangman's fracture with reabsorbed axis.

pedicle, underwent $\mathrm{C} 2$ body screw along with $\mathrm{C} 3 \mathrm{C} 4 \mathrm{PSRF}$ and $\mathrm{C} 2$ pedicle reconstruction.

CONCLUSION: This is the first series of pediatric hangman's fracture reported in world literature. In our center we have evolved in the direction of motion preservation at $\mathrm{C} 1 \mathrm{C} 2$ joint, along with 3 column stable fixation of the $\mathrm{C} 2$ pedicle, which is of paramount importance in the pediatric population with long life expectancy.

Keywords: Pediatric, hangmans fracture,Pedicle reconstruction

\section{FL-109}

\section{Spine \\ Management of Chiari malformation - individualized treatment and outcome}

Martina Messing Jünger ${ }^{1}$, Stephanie Jünger ${ }^{2}$, Andreas Röhrig $^{1}$, Jasmin Al Hourani ${ }^{1}$, Sandra Kunze ${ }^{1}$, Friederike Knerlich Lukoschus ${ }^{1}$

${ }^{1}$ Neurochirurgische Abteilung, Asklepios Klinik, Sankt Augustin, Germany

${ }^{2}$ Neurochirurgische Klinik, Universitätsklinik Köln, Köln, Germany

OBJECTIVE: The contemporary concept of Chiari malformation $(\mathrm{CM})$ comprises different conditions related to the underlying pathology (CM0, CM1, CM1.5 and CM2). Specific treatment options are offered according to the individual clinical condition. This clinical series reflects the variety of the Chiari spectrum.

MATERIAL-METHODS: This is a retrospective study of a consecutive single unit (5 surgeons) series of patients who 
underwent foramen magnum decompression between 2010 and 2019 due to CM. Demographic data, diagnostic findings, relevant comorbidities, surgical treatment and outcome data are presented.

RESULTS: A total of 96 patients have been operated. Mean age was 10 years (range $0.2-60$ y). 50 patients were male, 46 female. $48(50 \%)$ presented with additional syringomyelia at time of surgery, 36(37.5\%) with scoliosis. 11 patients required secondery surgeries. The following $\mathrm{CM}$ subtypes have been treated: CM0(4), CM1(47), CM1.5(27) and CM2(18). Associated syrinx and scoliosis were most often seen in CM2 patients $(67 \%, 83 \%)$. All CM0 patients underwent intradural management. In the CM1 group 25(53\%) received bone only decompression (11[41\%] in CM1.5). Additional instrumentation (instability) became necessary in 9 patients (1 CM1, 8 CM1.5). In the CM2 group, 6 bone only and 10 intradural procedures have been performed. The overall complication rate after complete follow up is $8.3 \%$, leading to secondary surgery in 2 cases. Resolution or improvement of symptoms could be observed in all patients, syringomyelia resolution in 11(32) patients (CM2 excluded). There was no statistical difference regarding CM type, treatment and outcome. Bone only decompression provides similar results in CM1, CM1.5 and CM2 compared to intradural procedures, but syrinx resolution is delayed.

CONCLUSION: Different CM subtypes require specific management. Bone only decompression seems to have similar results in respect of resolving symptoms, syringomyelia and scoliosis progress compared to intradural techniques. No severe complications occured. Secondary complication surgery became necessary in 2 patients only.

Keywords: Chiari malformation, syringomyelia, foramen magnum decompression, CM1, CM2, CM1.5

\section{FL-110}

\section{Spine}

\section{Tethered cord syndrome with normal conus level}

Natsumi Yamamura, Masahiro Nonaka, Akio Asai Kansei Medical University Neurosurgery

OBJECTIVE: Symptomatic tethered cord occurs even though the level of conus medullaris is in the normal position. In such cases, diagnosis of the tethered cord is difficult, and it often take times to confirm the diagnosis. And it is unclear whether improvement of symptoms can be obtained even if surgery is performed long after the onset of symptoms.

MATERIAL-METHODS: We experienced 11 cases of symptomatic tethered cord syndrome with normal conus level. In all cases, the conus medullaris was located above the bottom of the L2 vertebrae.

RESULTS: 7 patients had urinary incontinence, and 5 patients had sensorimotor disorders in the lower limbs. 2 patients had both symptoms. The remaining patient had repeated urinary tract infection. The average age at the time of surgery was 13.5 years and the onset of symptoms was 6.4 years. The average time from the onset of symptoms to the confirmation of the diagnosis was 7.2 years. Only one case was found to have T1-high intensity spot in the filum on the MRI and in the remaining 10 cases, no abnormality was found in the filum. To assess the tension of the filum, Lumbar MRI images of the supine and prone positions were taken. For the surgery, hemilaminectomy was performed, and subsequently, the filum was sectioned. 6 out of 7 patients who had incontinence before surgery has improved, and all patients who had sensorimotor disorders in the lower limb showed improvement. The patient with repeated urinary tract infection did not show changes after surgery.

CONCLUSION: To confirm the diagnosis of the tethered cord with normal conus level, lumbar MRI was useful to assess the tension of the abnormal filum. Furthermore, the surgery was expected to show a good results, even though the patients were symptomatic for a long time.

Keywords: Tethered cord, normal conus level, Lumbar MRI images of the prone

\section{FL-111}

Spine

\section{Spinal trauma in children}

Di Rocco Féderico, Dauleac Corentin, Kaba Djene Ibrahima, Alsereihi Motaz, Boukaka R Gerald, Beuriat Pierre Aurelien, Szathmari Alexandru, Mottolese Carmine Pediatric Neurosurgery, Hôpital Femme Mère Enfant, Hospices Civils de Lyon, Université de Lyon, Lyon, France

OBJECTIVE: Aim of this study is to describe the epidemiology of spine trauma in children and evaluate the timing and techniques of surgery when it was required.

MATERIAL-METHODS: A retrospective review of all patients admitted to our pediatric trauma center for spine traumas that required surgical management, between 2005 and 2016, was performed. All patients were followed with clinical and radiologic examination to evaluate the quality of fusion and realignment.

RESULTS: Seventy-three children, with a mean age of 14.1 years, were admitted. Spinal injuries were more common in the teenage group (14-18 years). The predominant etiology of spine injuries was motor vehicle collision (36\%). 
The spinal level of injury varied according to the age group: young children presented more cervical traumas $(\mathrm{P}<0.01)$, while teenagers presented more lumbar traumas. There were more fractures alone in the teenage group $(\mathrm{P}<0.005)$, while there were more luxations alone in the school-age group $(\mathrm{P}<0.05)$. Median timing for surgical intervention in patients with neurologic deficits was 1 day. Patients with posterior wall recession $>5 \mathrm{~mm}$ underwent surgical management quicker than patients with no posterior wall recession $(\mathrm{P}<0.03)$. At follow-up, out of the 37 patients with initial neurologic deficits, 14 had improved (38\%).

CONCLUSION: Diskoligament maturation is an essential concept in spine traumas in children, as well as in surgical management. Surgical procedures should be carried out as soon as possible when patients present with neurologic deficits. At the thoracolumbar junction, a 2-level up-and-down fixation is recommended.

Keywords: Spinal, trauma, children

\section{FL-112}

\section{Spine}

Surgical management patients with mucopolysaccharidosis-related spinal deformities

\section{Polina Ochirova ${ }^{1}$, Sergey Ryabykh ${ }^{2}$, Alexander Gubin ${ }^{3}$}

${ }^{1}$ Polina V. Ochirova - MD, spine surgeon, pediatric orthopedic surgeon, Ilizarov Center, Kurgan, Russia

${ }^{2}$ Sergey O. Ryabykh-MD, PhD, spine surgeon, pediatric orthopedic surgeon, Director of Division Axial skeleton and Neurosurgery, Ilizarov Center, Kurgan, Russia

${ }^{3}$ Alexander V. Gubin-MD, PhD, spine surgeon, pediatric orthopedic surgeon, Priorov Center, Moscow, Russia

OBJECTIVE: Spinal deformity in patients with mucopolysaccharidosis a problem requiring early treatment.

MATERIAL-METHODS: We analyzed 12 cases of mucopolysaccharidosis (MPS), treated in Ilizarov Center in 2012-2020. There were 1 patient with MPS IH-type, 7 patients with IVA-type and 4 patients with MPS type VI. The age of the patients ranged from 6 to 26 years-old (average age -10.3 years-old). All patients had spinal deformity with primary and/or secondary stenosis of the spinal canal. Various surgical treatments were used: 1) two staged surgery was perfomed in three patients with cervical myelopathy and scoliosis/kyphosis: one patient-C0-C5 decompression with occipitospondylodesis (OSD) firstly and dual growing rod construction secondly; two and three patients $\mathrm{C} 0-\mathrm{C} 4$ decompression with occipitospondylodesis (OSD) firstly and multilevel fixation secondly; 2) final fusion in 2 patients with kyphoscoliosis; 3 ) decompression at the stenosis level (cervical spine) with OSD in 7 patients with cervical myelopathy.

RESULTS: Scoliosis value was from $20^{\circ}$ to $75^{\circ} \mathrm{Cobb}$, kyphosis from $15^{\circ}$ to $82^{\circ} \mathrm{Cobb}$. Four patients had vertebral stenosis and myelopathy (Frankel C). All patients had pulmonary and cardiac dysfunctions: vital capacity of the lungs was from 21 to $50 \%$ and abnormal ECG. Imbalance in the frontal and sagittal planes was present in $60 \%$ of patients. Through surgical treatment correction of the kyphosis was by $68 \%$ and scoliosis by $85 \%$. In 4 (50\%) patients with spinal stenosis neurologic status improved to Frankel E.

CONCLUSION: Patients with MPS quite often have vertebral stenosis and myelopathy, in such patients with combined stenosis of the spinal canal, it is necessary to combine decompression and fusion.

Keywords: mucopolysaccharidosis, spinal deformity, scoliosis, kyphosis, occipitospondylodesis

\section{FL-113}

\section{Vascular \\ Establishing a reference centre for management of pae- diatric CNS arteriovenous malformations-experience of the first 5 years}

$\underline{\text { Ido Ben Zvi }}{ }^{1}$, Adam Rennie ${ }^{2}$, Fergus Robertson ${ }^{2}$, Vijeya Ganesan $^{3}$, Sanjay Bhate ${ }^{3}$, Greg James ${ }^{2}$

${ }^{1}$ Department of Neurosurgery, Great Ormond Street Hospital, London, UK

${ }^{2}$ Interventional Neuroradiology, Great Ormond Street Hospital, London, UK

${ }^{3}$ Department of Neurology, Great Ormond Street Hospital, London, UK

OBJECTIVE: Paediatric CNS arteriovenous malformations (AVMs) are rare but important pathologies. Regional units manage many low grade AVMs locally, more complex cases may require multidisciplinary management in a unit with expertise in microsurgery (MS), endovascular embolisation (EE), stereotactic radiosurgery (SRS) and combinations thereof. Following the appointment of the senior author in late 2014, we have developed such a centre.

MATERIAL-METHODS: Retrospective electronic chart review of all children with AVMs referred to the centre since it establishment (Jan 2015).

RESULTS: 74 consecutive children identified (45 male, 29 female) with a mean age at presentation of 9.7 years (range 0-17 years), 70 with brain AVM and 4 with spinal cord AVM. The most common presentation was with haemorrhage (51), followed by incidental (10), neurological signs (8), seizures (3) and headache (2). For brain AVMs, all 
Spetzler-Martin grades were represented (I, 12 children; II, 20; III, 23; IV, 11; V, 4). 7/74 children had undergone neurosurgical treatment prior to referral to our service. Following referral, 18 children (mostly high-grade, incidental AVM) were managed conservatively, whilst 37 had single modality treatment (9 MS, 7 EE, 21 SRS). 19 children (all S-M grade $\mathrm{III}+)$ required multimodality treatment $(6 \mathrm{MS}+\mathrm{EE}, 2$ $\mathrm{MS}+\mathrm{EE}+\mathrm{SRS}, 3 \mathrm{MS}+\mathrm{SRS}$ and $8 \mathrm{EE}+\mathrm{SRS})$. There were 7 re-bleeds ( 4 in conservative group, 1 post EE, 1 post SRS and 1 while awaiting treatment) and no mortality. Of the 56 treated children, angiographic cure has been achieved in 26 so far, with several still awaiting final angiography. The majority (57/74) had favourable mRS (0-2) outcomes.

CONCLUSION: A dedicated multidisciplinary paediatric neurovascular team can offer all modalities of treatment for CNS AVMs in children, with acceptable outcomes. Our status as a referral centre attracts a large number of higher grade AVMs which often require multimodality treatment.

Keywords: AVM, arteriovenous malformation, microsurgery, embolisation, stereotactic radiosurgery

\section{FL-114}

\section{Vascular \\ Surgical management cerebral and medullary caverno- mas in children}

Kaba Djene Ibrahima, Di Rocco Féderico, Boukaka Rel Gerald, Alsereihi Motaz, Szathmari Alexandru, Beuriat Pierre Aurelien, Mottolese Carmine

Pediatric Neurosurgery, Hôpital Femme Mère Enfant, Centre de Référence Craniosténoses, Hospices Civils de Lyon, Université de Lyon, Lyon, France

OBJECTIVE: We reviewed our experience in the surgical treatment of 58 children with cerebral and medullary cavernomas.

MATERIAL-METHODS: Since 2001, 58 children ( 35 boys and 23 girls) were operated for a cerebral and medullary cavernomas. The median of age at surgery is 11.7 years (range 8 months to 18 years; median 17.7). Clinical symptomatology was dominated by the haemorrhage in 27 cases (46\%) followed by epilepsy as first symptom in 12 cases. In 13 cases were multiple cerebral cavernomatosis which were hereditary in 7 cases. The localisation was supratentorial in 37 cases $(63.8 \%)$, at the level of the posterior fossa in 16 cases $(27.6 \%)$ and medullary in 5 cases $(8.6 \%)$. All the children had initial MRI workup before surgery.

RESULTS: Complete removal was possible in all cases, even for brainstem or motor region locations. For supratentorial cavernomes the outcome was good in all cases. 4 patients with brain stem cavernomas presented neurological deficits:
2 cases with VII nerve paresis (one complete) and one with persistent cerebellar syndrome. Epilepsy control was obtained in all cases and allowed withdrawal from antiepileptic treatment for all but one patient with cavernomatosis. CONCLUSION: Surgical treatment of central nervous system cavernomasis a safe option with a low morbidity with a good control in cases of epilepsy with the exception of cavernomas localized in the brainstem.

Keywords: cavernomas, children, management

\section{FL-115}

\section{Vascular}

Characteristics of Neonates and Infants on Spontaneous Intracranial Hemorrhage due to Coagulopathy in Dr. Hasan Sadikin Hospital Bandung: 2014-2019

Mochamad Sri Arya Heriawan, Mirna Sobana

Department of neurosurgery, university of padjadjaran, bandung, indonesia

OBJECTIVE: One of the most common causes of deaths in neonatus and infant is haematological abnormalities, including intracranial hemorrhage, which is equal to $6 \%$. Intracranial bleeding is usually accompanied by serious neurological sequelae and even death. In neonates, intracranial bleeding is often caused by coagulation abnormalities. The study was conducted to determine the characteristics of neonatal and infant patients in spontaneous intracranial hemorrhage caused by abnormalities of blood clotting disorders at Dr. Hasan Sadikin Hospital Bandung in 2014-2019

MATERIAL-METHODS: This research is a retrospective study conducted with descriptive methods of neonatal and infant patients in spontaneous intracranial hemorrhage caused by coagulation abnormalities at Hasan Sadikin Hospital in Bandung from 2014-2019. The sample selection is done by consecutive sampling technique and selected samples that meet the inclusion criteria. Data was collected through medical records and analyzed using SPSS for Windows version 20.

RESULTS: In this study there were 112 respondents. 32 were male respondents (28.5\%) and 80 were female respondents (71.5\%). Surgical and non-surgical intervention showed almost equal proportions, as many as 51 respondents $(45.5 \%)$ carried out surgical intervention and as many as 55 respondents $(54.5 \%)$ did not take surgical intervention. Most respondents, $98(87.5 \%)$ had a history of decreased awareness and $76(68.5 \%)$ had a history of seizures. The midline shift picture shows that 86 respondents $(76.8 \%)$ experienced midline shift. 
CONCLUSION: Intracranial hemorrhages cases mostly founded in female neonates and infant. Surgical interventions which had been carried out in the cases showed equal proportion. Most of the patients showed neurologic deficit as on of its clinical manifestation.

Keywords: intracranial hemorrhage, coagulation disorder, neonates, infant, neurologic deficit.

\section{Nursing Abstracts}

\section{NS-001}

\section{Nursing Symposium \\ Diagnostic intracranial pressure monitoring for paedi- atric shunted hydrocephalus patients}

\section{Jody Oconnor}

Department of neurosurgery, Great Ormond street hospital, London, UK

OBJECTIVE: To understand the benefits of ICP monitoring in shunted paediatric patients. The objectives were to identify parameters that guide ICP interpretation and practice by understanding patient outcome in terms of symptomatic resolution, rehospitalisation and procedural complications. MATERIAL-METHODS: Paediatric patients with shunted hydrocephalus, admitted for diagnostic ICP monitoring within a 4-year period were included. Retrospective analysis of ICP monitoring reports provided 3 groups of patients; overdrainage, underdrainage or within normal limits. The ICP reports were generated using ICM + software and interpreted by nurse practitioners with recommendations for intervention. Data was collated to determine the intervention groups; surgery, shunt reprogramming and medical management. Both reporting and intervention groups were analysed in terms of resolution of symptoms, rehospitalisation and complication rates using excel and SPSS.

RESULTS: No major complications related to ICP monitoring but $7.8 \%$ patients were treated for a superficial wound infection. $38 \%$ of patients were reported as shunt malfunction, $32 \%$ within normal limits, $65 \%$ patients had resolution of symptoms and $47 \%$ patients avoided surgery. Multiple spikes in ICP over $20 \mathrm{mmHg}$; negative or swings in ICP and multiple spikes in mean wave amplitude (MWA) are all good predictive parameters.

Patients reported as shunt malfunction were significantly more likely to undergo surgical treatment $P=0.0002$. Surgically treated patients did significantly better in terms of symptoms at both early and long-term follow up. Patients with ICP parameters within normal limits were less likely to be readmitted to the neurosurgical unit within 6 months.
Negative ICP parameters, and adherence to ICP reports impacted poorly on symptomatic outcomes for patients.

CONCLUSION: Patients benefit from ICP monitoring when shunt malfunction is in question, providing evidence to assist with clinical decision making. Better symptomatic outcomes, reduction in unnecessary shunt surgery and reduced readmission rates were all positive findings in this study.

Keywords: hydrocephalus, VP shunt, ICP monitoring, paediatric

\section{NS-002}

\section{Nursing Symposium}

An Argument for Specialized Neurosurgical Nursing in Low-and-Middle Income Countries: One Nurse's Experience

\section{Ashley K Birch}

Department of Neurosurgery, Boston Childrens Hospital, Boston, MA, USA

OBJECTIVE: To discuss the importance of specialized neurosurgical nursing globally, particularly in low-and-middle income countries (LMIC).

To compare the experience of caring for patients in LMIC at an institution with specialized neurosurgical care and one without specialized nursing.

MATERIAL-METHODS: A review of the literature was completed to accumulate all available scientific evidence regarding specialized nursing in the global context. The primary investigator visited two clinical sites and made objective assessments of the nursing care at each. Site 1: Mbale, Uganda was a small private not for profit pediatric neurosurgical facility with dedicated nursing staff heavily experienced in this patient population. Site 2: Dar Es Salaam, Tanzania, was a large government hospital housing multiple surgical services with a non-specialized nursing staff caring for multiple patient populations. The primary investigator conducted on-site interviews with nurses at both hospitals. RESULTS: The World Health Organization (WHO) Education Guidelines support investing in specialized training and education for the health care workforce. The literature suggests that specialized education contributes to improved quality and outcomes. In LMIC nurses are often rotated to different units with patients impacted by multiple conditions and this does not allow for promoting increased competence and expertise. The diagnosis, treatment and care of pediatric neurosurgical patients is complex and nurses providing care for these children benefit from specialized education and clinical training. The specialized nurses demonstrated extensive knowledge in the conditions and treatments of this population and reported higher job satisfaction. 
CONCLUSION: The World Health Organization supports the specialized education of the healthcare workforce globally and particularly in LMIC. Improved quality of care and outcomes are noted when healthcare workers are given the opportunity to specialize. As nurses are increasingly recognized as leaders in healthcare globally the focus should be on supporting specialized neurosurgical training and education to promote increased safety and competency for this patient population.

Keywords: pediatric neurosurgery, low-and middle-income, nursing, safety, specialized education

\section{NS-003}

\section{Nursing Symposium \\ Freeze preservation of cranial bones after decompressive craniectomy for future cranioplasty. Our experience}

\author{
Martinez Gonzalez Karolina ${ }^{1}$, Garcia Gonzalez Oscar ${ }^{2}$ \\ ${ }^{1}$ Universidad Autonoma de Aguascalientes \\ ${ }^{2}$ Hospital Regional de Alta Especialidad del Bajio
}

OBJECTIVE: Decompressive craniectomy is routinely performed to treat intracranial hypertension refractory to medical therapy as a result of head trauma, cerebrovascular accident (CVA) or brain tumors. It is later followed by a cranioplasty procedure (CP) in the surviving patients, with autologous bone graft or other reconstructive materials is considered to repair the skull defect.

This retospective study reviews the cases of decompressive craniectomies followed by later cranioplasty undertaken the experience since 2013 and describes the method used for preservation of removed bone flaps for future cranioplasty. To clarify the clinical outcomes of cranioplasty with cryopreserved bone flaps and identify risk factors related to bone flap infection and resorption after cranioplasty with cryopreserved bone flaps. MATERIAL-METHODS: 5 patients (4 children) underwent decompressive craniotomies and delayed autologous cranioplasty since 2013. A protocol was designed to prepare the removed bone flaps for freeze preservation. After removal, the bone flaps were transferred to the blood bank of our institution within $6 \mathrm{~h}$, gently rinsed using 1-3 L of sterile saline $(0.9 \% \mathrm{NaCl})$ supplemented with antibiotics (amikacyn), then flaps were wrapped in two layers of sterile plastic coverage and preserved at $-20 / 25^{\circ} \mathrm{C}$.

RESULTS: The patient's population will be presented. Since 2013 we have performed 5 cranioplasties using freeze preserved autologous bone graft. The median time from craniectomy to cranioplasty was 85 days. Up to now, no case of infection, osteomyelitis or complete bone resorption following cranioplasty have occurred.

CONCLUSION: Freeze preservation of autologous bone grafts to reconstruct skull defects after decompressive craniectomy is a useful procedure and has a low revision or complication rate and led to the unnecessary use of synthetic prostheses and increased health care costs.

Keywords: freeze preservation, bone flaps, craniotomy

\section{NS-004}

\section{Nursing Symposium}

The effects of administering laxatives early in the post operative stage for patients with spinal lipomas undergoing spinal cord untethering surgery

\section{Oliver Joseph Hutley \\ Great Ormond Street Hospital, London, UK}

OBJECTIVE: To review and discuss the effects of commencing laxatives early in the post operative stage for patients who have undergone spinal cord untethering surgery. Identify whether early administration of laxatives can improve recovery time and subsequently discharge time. MATERIAL-METHODS: This case study examines data from 50 patients. The reviewed data includes fluid balance charts, pain assessments, drug charts and documentation from nurses, doctors and the wider multidisciplinary team. RESULTS: Yet to be identified and therefore will be presented during the conference.

CONCLUSION: Patients in the above population often require the administration of osmotic laxatives post operatively. However, often there are delays with prescribing and/ or administering these medicines and therefore patients may not pass a bowel motion for several days into their recovery. The effects of regular opioids and lack of mobilisation exacerbate these inabilities also, and therefore may hinder the recovery time.

Pain and discomfort caused by constipation additionally creates delays for patients recovering. They may become less engaged with physiotherapy, more reluctant to mobilise and reduce their dietary intake, again slowing down recovery progress and ultimately a patients discharge.

Keywords: Laxatives, Spinal Surgery, Nursing

\section{NS-005}

Nursing Symposium

Lived Experience of Mothers of Children with Spina Bifida

Manju Dhandapani ${ }^{1}$, Shivasnanmugam Dhandapani ${ }^{2}$

${ }^{1}$ National Institute of Nursing Education, PGIMER, Chandigarh, India 
${ }^{2}$ Department of Neurosurgery, PGIMER, Chandigarh, India

OBJECTIVE: To assess the lived experience of mothers of children with spina bifida.

MATERIAL-METHODS: A qualitative study was conducted on 12 mothers whose child was operated for correction of spina bifida. A semi structured interview was conducted with the mothers by the researcher. The information collected were under themes such as 'reaction at the time of diagnosis', 'Acceptance of child's condition', 'present physical, psychological and social well-being', impact on work and activities of daily living', and 'the future concern about the child'.

RESULTS: The findings are described under each theme. Reaction at the time of diagnosis: Mothers expressed that they experienced emotional distress, denial and hopelessness at the time of diagnosis.

Acceptance of child's condition: The condition of the child was accepted by mothers over a period of time with the information provided by the health care providers and even counseling.

Present physical, psychological and social well-being: Poor psychological and social well being was reported more as compared to physical problems. Few mothers reported fatigue and headache due to stress and constant caregiving. Impact on work and activities of daily living: Mothers also reported that their ADL and work schedule is affected due to hospital visits and also due to care of the child.

The Future concern about the child: Many mothers expressed concern about the adverse impact of spina bifida on growth and development, especially in the area of ambulation, muscle weakness, cognitive development, bowel and bladder control and familial transmission of the condition.

CONCLUSION: Mothers of children with spina bifida experience substantial amount of burden since the time of diagnosis and more so they continue to experience the same during the growth and development of the child. Appropriate assessment of the burden of parents can aid in appropriate interventions by the nurses, doctors and other health care providers.

Keywords: spina bifida, caregiver burden, quality of life, experiences

\section{NS-006}

\section{Nursing Symposium}

\section{Application of the custom-made preparation program}

Toshiko Momokawa ${ }^{1}$, Yusaku Kikuchi ${ }^{1}$, Miyuki Inagaki ${ }^{1}$, Yoshimi Tanase ${ }^{2}$, Mihoko Kato ${ }^{3}$

${ }^{1}$ Faculty of Nursing, Aichi Children's Health and Medical Center, Aichi, Japan

${ }^{2}$ Child-care service, Aichi Children's Health and Medical Center, Aichi, Japan
${ }^{3}$ Department of Neurosurgery, Aichi Children's Health and Medical Center, Aichi, Japan

OBJECTIVE: The Convention on the Rights of the Child guarantees the child's right to receive reasonable explanation according to individual development.

In this report, we introduce a custom-made preparation program tailored to each patient.

MATERIAL-METHODS: [Details1].

Our nursing team usually copes with Hospital Play Specialists (HPS) to perform preparation for examinations and treatments. In standard program, we use picture-books and dolls that play patients' rolls and receive various examinations and treatments to enable patients to understand.

In order to upgrade, we used "Sugoroku" that is a classic children's board game in Japan similar to Monopoly. In the Sugoroku, typical clinical steps were drown in many squares and a player (a patient) moves on clinical course step-by-step with throwing a dice. Playing Sugoroku, patients understand their own disease, examinations and treatments they were receiving and reduce their anxiety.

RESULTS: [Details2].

In first step, HPS creates Sugoroku of the original, it is revised the contents in multidisciplinary conference.

While playing Sugoroku with patients, multidisciplinary team assess patient's comprehension of the disease and gauge anxieties.

In second step, based on the several information got from patient's reactions playing Sugoroku, we set up the custommade preparation program.

In third step, we apply the program to patient. And the patient's mental information obtained from the custom-made preparation program was shared between multidisciplinary team. We feedback the patient's mental condition to daily nursing care.

CONCLUSION: Using Sugoroku, we were able to notice the degree of patient's understanding and anxious points. Information from Sugoroku is useful to create the upgrade program. In addition, using the custom-made preparation program, the patients deepened their understanding of the disease. We could offer more heartful nursing care.

Keywords: custom-made preparation, Sugoroku

\section{NS-007}

\section{Nursing Symposium}

Perioperative nursing experience of $3 \mathrm{D}$ printed polyether ether ketone repair materials in the treatment of pediatric skull defect repairment

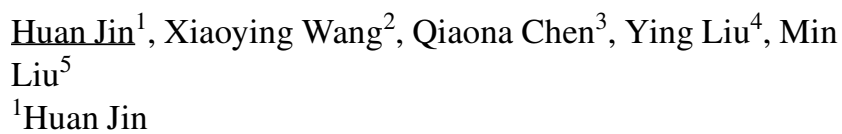


${ }^{2}$ Xiaoying Wang

${ }^{3}$ Qiaona Chen

${ }^{4}$ Ying Liu

${ }^{5}$ Min Liu

OBJECTIVE: To explore the perioperative nursing points of pediatric skull defect repairment using 3D printed polyether ether ketone (PEEK) as repair material.

MATERIAL-METHODS: The study retrospectively summarized clinical data and the perioperative nursing process and main nursing measures of 27 pediatric patients undergoing skull repair using 3D printed polyether ether ketone as repair material from January 2018 to December 2019.

RESULTS: The skull defects of all the 27 children (3-14 years old, mean 7.6 years old, skull defect from 3 months to 2 years old) were repaired satisfactorily by comparing with the results of three-dimensional CT reconstruction before and after surgery. The appearance of the skulls of all children was restored after the repairment, and they were satisfied with the aesthetic degree. One child had transient subcutaneous skin flap effusion after surgery, which disappeared after puncture. During the follow-up from 3 to 6 months after discharge, all the children had no complications such as exposure of the patch, displacement of the patch and rejection.

CONCLUSION: Repairment of kull defect with 3D printing PEEK as repair material has satisfaction in aesthetic degree and few complications. It is important to observe the changes of the condition, detect the complications early, and carry out targeted perioperative nursing (posture management, diet nursing, wound nursing, psychological nursing, health education, etc.)for early recovery..

Keywords: Polyetheretherketone (PEEK); 3D printing; Cranial defect repair in children; Perioperative nursing

\section{NS-008}

\section{Nursing Symposium \\ Nursing care of children with epilepsy after stereotactic electroencephalogram}

Qiaona Chen ${ }^{1}$, Huan $\mathrm{Jin}^{2}$, Fang $\mathrm{Gao}^{3}$, Munqun $\mathrm{Liu}^{4}$, Ming $\mathrm{Liu}^{5}$, Ying Liu ${ }^{6}$, Youzhen Sun ${ }^{7}$

${ }^{1}$ Qiaona Chen

${ }^{2}$ Huan Jin

${ }^{3}$ Fang Gao

${ }^{4}$ Munqun Liu

${ }^{5}$ Min Liu

${ }^{6}$ Ying Liu

${ }^{7}$ Youzhen Sun

OBJECTIVE: observation and nursing of children after prevention of postoperative complications, ensure the safety of children,provide more accurate etiology of epileptogenic focus for surgeons.

MATERIAL-METHODS: combining the nursing routine of epilepsy surgery with the purpose of SEEG.our department has carried out close observation and professional nursing on more than 150 children under the age of 10with SEEG. RESULTS: Accurate localization of epileptogenic focus by SEEG in more than 150 children.

CONCLUSION: Do a good job of relevant nursing. Aroid the symptoms of accidental injury, aspiration and anoxia during the attack promote the smooth operation and rehabilitation of children.

Keywords: epilepsy,SEEG, aspiration,accidental injury,nursing

\section{NS-009}

\section{Nursing Symposium \\ Application of $6 \mathrm{~S}$ management in perioperative nursing of children with cerebral palsy}

\author{
$\underline{\text { Munqun Liu }}^{1}$, Huan $\mathrm{Jin}^{2}$, Fang $\mathrm{Gao}^{3}$, Yan Zhang ${ }^{4}$, Min Liu ${ }^{5}$, \\ Ying Liu ${ }^{6}$, Youzhen Sun \\ ${ }^{1}$ Muqun Liu \\ ${ }^{2}$ Huan Jin \\ ${ }^{3}$ Fang Gao \\ ${ }^{4}$ Yan Zhang \\ ${ }^{5}$ Min Liu \\ ${ }^{6}$ Ying Liu \\ ${ }^{7}$ Youzhen Sun
}

OBJECTIVE: To investigate the effect of $6 \mathrm{~S}$ management in perioperative nursing of children with cerebral palsy.

MATERIAL-METHODS: Eighty children with cerebral palsy were enrolled and divided into observation group and control group according to the random number table method, 40 cases in each group. All patients underwent peripheral nerve selective partial resection (SPN). During the perioperative period, the control group was administered the general management method, and the observation group was administered the $6 \mathrm{~S}$ management method. The hospitalization satisfaction of the two groups of children with cerebral palsy was observed, and the cerebral palsy scale was scored at the time of admission and before discharge.

RESULTS: The cerebral palsy scales of the two groups were similar when they were admitted to the hospital. The difference was not statistically significant $(\mathrm{P}>0.05)$. After the $6 \mathrm{~S}$ management intervention, the pre-discharge cerebral palsy scale score was significantly higher than that of the control group $(\mathrm{P}<0.05)$, hospitalization satisfaction was significantly higher than the control group $(\mathrm{P}<0.05)$. 
CONCLUSION: The application of $6 \mathrm{~S}$ management in the perioperative nursing of children with cerebral palsy can reduce the degree of disability in children, improve their daily living ability, and improve the hospitalization satisfaction of patients.

Keywords: 6S management; cerebral palsy; perioperative period.

\section{NS-010}

\section{Nursing Symposium}

Standardized system for prevention of aspiration in critically ill children with cerebral hemorrhage in neurosurgery

Xiaoyu $\mathrm{Ma}^{1}$, Huan $\mathrm{Jin}^{2}$, Hou Dan ${ }^{3}$, Huan $\mathrm{Xu}^{4}$, Yajing Zhang ${ }^{5}$, Hongrui $\mathrm{Li}^{6}$, Jing Ban ${ }^{7}$

${ }^{1}$ Xiaoyu Ma

${ }^{2}$ Huan Jin

${ }^{3}$ Hou Dan

${ }^{4} \mathrm{Huan} \mathrm{Xu}$

${ }^{5}$ Yajing Zhang

${ }^{6}$ Hongrui LI

${ }^{7}$ Jing Ban

OBJECTIVE: to establish a systematic nursing system in neurosurgery to prevent aspiration of children with tracheal intubation, so as to ensure the safety of children.

MATERIAL-METHODS: To review the risk factors of aspiration in children, and to establish a standardized system to prevent aspiration in combination with the characteristics of Neurosurgery diseases, which was applied in clinical practice from January 2018 to January 2020.

RESULTS: The incidence of aspiration and aspiration pneumonia was compared before and after the implementation of anti aspiration standardization system, and the probability of aspiration and aspiration pneumonia was compared before and after the implementation of anti aspiration standardization system. Statistical software SPSS18.0 was used to analyze the data of the two groups. Chi square test was used to count the data, and $\alpha=0.05$ was used as the test level. Dysphagia in children with cerebral hemorrhage in neurosurgery was a serious risk factor for aspiration pneumonia. CONCLUSION: By using the standardized system of preventing aspiration, nurses can effectively grasp the relevant preventive measures, so as to avoid aspiration of critical children with cerebral hemorrhage in neurosurgery to the greatest extent, and provide a guarantee for the safety of patients.

Keywords: Neurosurgery; cerebral hemorrhage critical children; prevention of aspiration; standardized system
NS-011

\section{Nursing Symposium}

Clinical effectiveness and family experience of telephonic consultation in a regional pediatric neurosurgery center in the United Kingdom

William B Lo, Katie Elizabeth Herbert, Desiderio Rodrigues Department of Neurosurgery, Birmingham Women's and Children's NHS Trust, Birmingham, United Kingdom

BACKGROUND AND AIM:Pediatric neurosurgery outpatient consultation is conducted face-to-face (FTF) conventionally. Reasons for not utilizing telemedicine include the perceived difficulty to obtain reliable history and lack of physical examination. However, FTF consultation can cause distress and inconvenience. We piloted the nurse specialist-led telephonic consultation (TC) for follow-up appointments, extending to the routine neurosurgeon clinics in 2020 . This study evaluates the effectiveness of TC, families' experience of TC $\mathrm{v}$ traditional FTF appointments, and factors associated with their preferences. METHODS:The prospective study, using survey methodology, TCs by 3 clinicians over 8 weeks were evaluated. Based on clinical background, each patient was assigned a TC or FTF appointment. Clinical, surgical details and home postcode were recorded. At the end of each TC, the clinician recorded whether the child required a FTF appointment within 3 months. Patients/ families answered: how the current TC compared to FTF consultation, and their preference for future consultation.

RESULTS: 114 TCs were included. No children required a FTF appointment within 3 months. Overall, compared to FTF, TC was 'same/better/much better' for 101 families (89\%), and 'worse/much worse' for 13 (11\%). 2/3 of families preferred the next appointment as TC. Families attending TC as new appointments preferred the next appointment to be FTF more than follow up TCs (75\% v $29 \%)$. A high rating of the current $\mathrm{TC}$ was associated with a preference for future TC appointment. Families preferring TC over FTF lived further away (mean 38 v $27 \mathrm{~km}$ ).

CONCLUSIONS:TC is adequate in appropriately selected patients as either the primary mode of consultation or as a triage system. From patient perspective, the majority of families felt it was the same/better than traditional FTF appointments. The findings suggested that: new patients should be offered FTF appointments; follow-up TC should be offered to families; clinicians should develop their skills in conducting TC.

Keywords: Telemedince, telephonic consultation, pediatric neurosurgery, COVID 


\section{E-Poster List}

\begin{tabular}{|c|c|c|c|c|}
\hline Pub Number & Topic & Abstract Title & Presenter & Country \\
\hline P-001 & Dysraphism & $\begin{array}{l}\text { Case Report: A Huge Lipomy- } \\
\text { elomeningocele }\end{array}$ & Ade Ricky Harahap & Indonesia \\
\hline P-002 & Dysraphism & $\begin{array}{l}\text { Enlargement of spinal lipoma } \\
\text { in early postnatal period }\end{array}$ & Kazuhisa Yoshifuji & Japan \\
\hline P-003 & Dysraphism & $\begin{array}{l}\text { Diastematomyelia type I asso- } \\
\text { ciated with intramedullary } \\
\text { lipoma and dermoid cyst. An } \\
\text { extremely rare case report }\end{array}$ & Katerina Apostolopoulou & United Kingdom \\
\hline P-004 & Dysraphism & $\begin{array}{l}\text { Prevalence of sleep apnea in } \\
\text { children with myelomenin- } \\
\text { gocele }\end{array}$ & Brandon George Rocque & United States \\
\hline P-005 & Dysraphism & $\begin{array}{l}\text { Factors affecting QOL fol- } \\
\text { lowing surgery for Spinal } \\
\text { Dysraphism in Children }\end{array}$ & Sivashanmugam Dhandapani & India \\
\hline P-006 & Dysraphism & $\begin{array}{l}\text { Epidemiological and clinical } \\
\text { aspects of \{ Spina Bifida }\} \text { and } \\
\text { surgical management limits } \\
\text { in a third-world country: } \\
\text { report of a series of } 30 \text { cases } \\
\text { and review of the literature }\end{array}$ & Meissa Hamza & Tunisia \\
\hline P-007 & Dysraphism & $\begin{array}{l}\text { Surgery for Spinal Occult Dys- } \\
\text { raphism in a non-pediatric } \\
\text { hospital }\end{array}$ & Abraham Ibarra De La Torre & Mexico \\
\hline P-008 & Dysraphism & $\begin{array}{l}\text { Multiple-site neural tube } \\
\text { defects: Embryology and } \\
\text { Management strategies }\end{array}$ & Dwarakanath Srinivas & India \\
\hline P-009 & Dysraphism & $\begin{array}{l}\text { Multi Stage Procedures For } \\
\text { Frontoethmoidal Mengin- } \\
\text { goencephaloceles }\end{array}$ & Muhammad Azhary Lazuardy & Indonesia \\
\hline P-010 & Dysraphism & $\begin{array}{l}\text { Neonatal chyloperitoneum } \\
\text { following open myelomenin- } \\
\text { gocele repair: Dealing with a } \\
\text { rare finding }\end{array}$ & Ioannis N Mavridis & United Kingdom \\
\hline P-011 & Dysraphism & $\begin{array}{l}\text { Predicting neurological } \\
\text { outcome after surgery for } \\
\text { tethered cord based on } \\
\text { intraoperative neuromonitor- } \\
\text { ing outputs: How close can } \\
\text { we get? }\end{array}$ & Suhas Udayakumaran & India \\
\hline P-012 & Dysraphism & $\begin{array}{l}\text { Open neural tube defects } \\
\text { associated with split cord } \\
\text { malformation }\end{array}$ & Ai Muroi & Japan \\
\hline P-013 & Dysraphism & $\begin{array}{l}\text { Cerebrospinal Fluid Leakage } \\
\text { and Meningitis after Resec- } \\
\text { tion of the Cervical Skin Tag }\end{array}$ & Inthira Khampalikit & Thailand \\
\hline P-014 & Dysraphism & $\begin{array}{l}\text { Our experience in treating chil- } \\
\text { dren with fixed spinal cord in } \\
\text { Currarino syndrome }\end{array}$ & Pavel Lobankin & Russia \\
\hline P-015 & Dysraphism & $\begin{array}{l}\text { Dysraphisms: towards a new } \\
\text { European Classification }\end{array}$ & Timothée De Saint Denis & France \\
\hline P-016 & Epilepsy & $\begin{array}{l}\text { The occurrence and risk fac- } \\
\text { tors of new-onset seizures } \\
\text { after ventricular shunting } \\
\text { procedures }\end{array}$ & Mohammad Sadegh Masoudi & Iran \\
\hline
\end{tabular}




\begin{tabular}{|c|c|c|c|c|}
\hline Pub Number & Topic & Abstract Title & Presenter & Country \\
\hline$\overline{\mathrm{P}-017}$ & Epilepsy & $\begin{array}{l}\text { Seizure-outcome of Long-term } \\
\text { Epilepsy Associated Tumors } \\
\text { (LEATs) in Pediatric and } \\
\text { young adolescents }\end{array}$ & Suyash Singh & India \\
\hline P-018 & Epilepsy & $\begin{array}{l}\text { Real-time MR Thermography- } \\
\text { guided Laser Ablation for } \\
\text { Hypothalamic Hamartomas } \\
\text { in Pediatric Patients }\end{array}$ & Santiago Candela Cantó & Spain \\
\hline P-019 & Epilepsy & $\begin{array}{l}\text { Real-time thermography- } \\
\text { guided laser ablation to com- } \\
\text { plete disconnective surgeries } \\
\text { for refractory epilepsy in } \\
\text { pediatric patients }\end{array}$ & Santiago Candela Cantó & Spain \\
\hline P-020 & Epilepsy & $\begin{array}{l}\text { Stereotaxic biopsy prior to } \\
\text { laser ablation of lesions } \\
\text { causing refractory epilepsy in } \\
\text { pediatric patients }\end{array}$ & Santiago Candela Cantó & Spain \\
\hline P-021 & Epilepsy & $\begin{array}{l}\text { Focal Resection for epilepsy } \\
\text { surgery in the very young }\end{array}$ & William Lo & United Kingdom \\
\hline P-022 & Epilepsy & $\begin{array}{l}\text { Determinants of Seizure } \\
\text { Freedom in SEEG-guided } \\
\text { Resective Epilepsy Surgery: } \\
\text { Does it matter how much you } \\
\text { resect? }\end{array}$ & Mehdi Khan & United Kingdom \\
\hline P-023 & Epilepsy & $\begin{array}{l}\text { The efficacy of corpus callo- } \\
\text { sotomy for epileptic spasms: } \\
\text { analysis of phase amplitude } \\
\text { coupling }\end{array}$ & Yasushi Iimura & Japan \\
\hline P-024 & Epilepsy & $\begin{array}{l}\text { Role of autostimulation } \\
\text { responding to ictal tachycar- } \\
\text { dia in VNS therapy for medi- } \\
\text { cally refractory epilepsy }\end{array}$ & Takamichi Yamamoto & Japan \\
\hline $\mathrm{P}-025$ & Epilepsy & $\begin{array}{l}\text { Surgical Treatment of Epilepsy } \\
\text { in Children with Sturge- } \\
\text { Weber Syndrome with focus } \\
\text { on focal lesionectomy: A } \\
\text { Case-Based Review }\end{array}$ & Nicole Frank & Switzerland \\
\hline P-026 & Functional & $\begin{array}{l}\text { Quick protocol MRI without } \\
\text { sedation for presurgical } \\
\text { identification of the conus } \\
\text { medullaris in selective dorsal } \\
\text { rhizotomy patients }\end{array}$ & Leopold Arko Iv & United States \\
\hline P-027 & Germ Cell Tumors & $\begin{array}{l}\text { Craniospinal irradiation is nec- } \\
\text { essary for primary intramed- } \\
\text { ullary yolk sac tumor - Case } \\
\text { Report and Review of } \\
\text { Literature }\end{array}$ & Jimmy Ming Jung Chuang & Taiwan \\
\hline P-028 & Germ Cell Tumors & $\begin{array}{l}\text { Treatment strategies for giant } \\
\text { immature teratomas in early } \\
\text { infants }\end{array}$ & Tomoru Miwa & Japan \\
\hline P-029 & Germ Cell Tumors & $\begin{array}{l}\text { Intracranial germinoma origi- } \\
\text { nating from lateral ventricle } \\
\text { with atypical symptom }\end{array}$ & Yuki Kuranari & Japan \\
\hline P-030 & $\begin{array}{l}\text { Global Pediatric Neu- } \\
\text { rosurgery }\end{array}$ & $\begin{array}{l}\text { Surgical treatment of the } \\
\text { lipomeningocele in children. } \\
\text { A review }\end{array}$ & Olim Akramov Zaribovich & Russia \\
\hline
\end{tabular}




\begin{tabular}{|c|c|c|c|c|}
\hline Pub Number & Topic & Abstract Title & Presenter & Country \\
\hline P-031 & $\begin{array}{l}\text { Global Pediatric Neu- } \\
\text { rosurgery }\end{array}$ & $\begin{array}{l}\text { Our experience and long term } \\
\text { results in case with extreme } \\
\text { macrocephaly with Dandy } \\
\text { Walker syndrome }\end{array}$ & Mirchevski Vladimir & Macedonia \\
\hline P-032 & $\begin{array}{l}\text { Global Pediatric Neu- } \\
\text { rosurgery }\end{array}$ & $\begin{array}{l}\text { Perioperative near-infrared } \\
\text { spectroscopy cerebral oxy- } \\
\text { genation saturation in pediat- } \\
\text { ric hydrocephalus patients in } \\
\text { a low resource setting }\end{array}$ & Jo Ling Goh & United States \\
\hline P-033 & $\begin{array}{l}\text { Global Pediatric Neu- } \\
\text { rosurgery }\end{array}$ & $\begin{array}{l}\text { Multi-directional Cranial } \\
\text { Distraction Osteogenesis } \\
\text { MCDO and other procedures } \\
\text { for sagittal synostosis }\end{array}$ & Masahiro Kameda & Japan \\
\hline P-034 & $\begin{array}{l}\text { Global Pediatric Neu- } \\
\text { rosurgery }\end{array}$ & $\begin{array}{l}\text { Pediatric brain and spinal } \\
\text { tumor presentation and man- } \\
\text { agement: experience from } \\
\text { a national referral center in } \\
\text { Bangladesh }\end{array}$ & Michael C. Dewan & Canada \\
\hline P-035 & $\begin{array}{l}\text { Global Pediatric Neu- } \\
\text { rosurgery }\end{array}$ & $\begin{array}{l}\text { Intracerebral Abscess In Patient } \\
\text { With Eisenmenger Syndrome }\end{array}$ & Arif Pebrianto Pebrianto & Indonesia \\
\hline P-036 & $\begin{array}{l}\text { Global Pediatric Neu- } \\
\text { rosurgery }\end{array}$ & $\begin{array}{l}\text { Paediatric Meningiomas in } \\
\text { Unusual Intracranial Loca- } \\
\text { tions: A Report of Three } \\
\text { Filipino Cases }\end{array}$ & Jeanne Vyka Fugaban Sarangay & Philippines \\
\hline P-037 & $\begin{array}{l}\text { Global Pediatric Neu- } \\
\text { rosurgery }\end{array}$ & $\begin{array}{l}\text { Clinical spectrum of amniotic } \\
\text { band syndrome and their } \\
\text { importance in neurosurgery: } \\
\text { A case report and literature } \\
\text { review }\end{array}$ & Cut Vanessa Rachmadian & Indonesia \\
\hline P-038 & $\begin{array}{l}\text { Global Pediatric Neu- } \\
\text { rosurgery }\end{array}$ & $\begin{array}{l}\text { Sellar Teratoma in a Child - } \\
\text { Case Report and Review of } \\
\text { the Literature }\end{array}$ & Katja Kuerner & Switzerland \\
\hline P-039 & Hydrocephalus & $\begin{array}{l}\text { ETV and CPC for the treatment } \\
\text { of hydrocephalus. Is there } \\
\text { a role for plexectomy in the } \\
\text { success outcome? How we } \\
\text { do it }\end{array}$ & Garcia Gonzalez Oscar & Mexico \\
\hline P-040 & Hydrocephalus & $\begin{array}{l}\text { Iatrogenic occipital intradiploic } \\
\text { and cervical intraosseous } \\
\text { CSF collections - why do } \\
\text { they develop and what we } \\
\text { can learn }\end{array}$ & Arthur Robert Kurzbuch & Switzerland \\
\hline P-041 & Hydrocephalus & $\begin{array}{l}\text { Outcome and Complications of } \\
\text { Endoscopic Third Ventriculo- } \\
\text { stomy for Obstructive Hydro- } \\
\text { cephalus: Experience at Lady } \\
\text { Reading Hospital }\end{array}$ & Taimoor Ali & Pakistan \\
\hline P-042 & Hydrocephalus & $\begin{array}{l}\text { Suprahepatic space as an alter- } \\
\text { native site for distal catheter } \\
\text { insertion in pseudocyst-asso- } \\
\text { ciated ventriculoperitoneal } \\
\text { shunt malfunction }\end{array}$ & Zohreh Habibi & Iran \\
\hline P-043 & Hydrocephalus & $\begin{array}{l}\text { ETV in infancy and childhood } \\
\text { below } 2 \text { years of age }\end{array}$ & Ahmed El Damaty & Germany \\
\hline P-044 & Hydrocephalus & $\begin{array}{l}\text { 30-Day Outcomes After } \\
\text { Intervention For Post-Hem- } \\
\text { orrhagic Hydrocephalus in } \\
\text { Preterm Infants: A NSQIP-P } \\
\text { Analysis }\end{array}$ & Shobhan Vachhrajani & United States \\
\hline
\end{tabular}




\begin{tabular}{|c|c|c|c|c|}
\hline Pub Number & Topic & Abstract Title & Presenter & Country \\
\hline$\overline{\mathrm{P}-045}$ & Hydrocephalus & $\begin{array}{l}\text { Management of Suprasellar } \\
\text { Arachnoid Cysts in Children: } \\
\text { A Systematic Literature } \\
\text { Review Highlighting Modern } \\
\text { Endoscopic Approaches }\end{array}$ & Christopher Bonfield & United States \\
\hline P-046 & Hydrocephalus & $\begin{array}{l}\text { Medico-economic aspects of } \\
\text { the management of childhood } \\
\text { hydrocephalus in the neu- } \\
\text { rosurgery department of the } \\
\text { Teaching hospital of Bouake } \\
\text { (Côte d'Ivoire) }\end{array}$ & Broalet Maman You Espérance & Cote d'Ivoire \\
\hline P-047 & Hydrocephalus & $\begin{array}{l}\text { Pediatric nonresponsive hydro- } \\
\text { cephalus. When should we } \\
\text { stop trating? }\end{array}$ & Dan Aurel Nica & Romania \\
\hline P-048 & Hydrocephalus & $\begin{array}{l}\text { Delayed bolus arrival time } \\
\text { drives global cerebral } \\
\text { hypoperfusion in paediatric } \\
\text { obstructive hydrocephalus }\end{array}$ & Sebastian M Toescu & United Kingdom \\
\hline P-049 & Hydrocephalus & $\begin{array}{l}\text { A Thirteen Year Retrospective } \\
\text { Review of Arachnoid Cysts in } \\
\text { Children }\end{array}$ & Fozia Saeed & United Kingdom \\
\hline P-050 & Hydrocephalus & $\begin{array}{l}\text { Progressive macrocrania } \\
\text { revealing a cerebral venous } \\
\text { thrombosis on a \{Dandy- } \\
\text { Walker\} syndrome: }\{\text { A case } \\
\text { report\} }\end{array}$ & Meissa Hamza & Tunisia \\
\hline P-051 & Hydrocephalus & $\begin{array}{l}\text { Factors of scheduled shunt } \\
\text { withdrawal failure }\end{array}$ & Mihoko Kato & Japan \\
\hline $\mathrm{P}-052$ & Hydrocephalus & $\begin{array}{l}\text { Deliberate endoscopic third } \\
\text { ventriculostomy for hydro- } \\
\text { cephalus in infants. Towards } \\
\text { a subsequent ventriculop- } \\
\text { eritoneal shunt morbidity } \\
\text { reduction? }\end{array}$ & Matthieu Helleringer & France \\
\hline P-053 & Hydrocephalus & $\begin{array}{l}\text { Time-To-Shunt in Mye- } \\
\text { lomeningocele-associated } \\
\text { Hydrocephalus in Dr. } \\
\text { Soetomo General Academic } \\
\text { Hospital, Indonesia }\end{array}$ & Rizki Meizikri & Indonesia \\
\hline $\mathrm{P}-054$ & Hydrocephalus & $\begin{array}{l}\text { Giant hydrocephalus: A study } \\
\text { of its gravity, challenges and } \\
\text { outcomes }\end{array}$ & Rohan Dilip Digarse & India \\
\hline P-055 & Hydrocephalus & $\begin{array}{l}\text { "Possible Shunt Malfunc- } \\
\text { tion" Pathway for Paediatric } \\
\text { Hydrocephalus - A Study of } \\
\text { Clinical Outcomes and Cost } \\
\text { Implications }\end{array}$ & Liting Tong & United Kingdom \\
\hline P-056 & Hydrocephalus & $\begin{array}{l}\text { Post Operative Seizure after } \\
\text { First Time Endoscopic Third } \\
\text { Ventriculostomy in Pediatric } \\
\text { Patients }\end{array}$ & Ariana Barkley & United States \\
\hline P-057 & Hydrocephalus & $\begin{array}{l}\text { Low or negative pressure } \\
\text { hydrocephalus in complex } \\
\text { paediatric posterior fossa } \\
\text { tumour }\end{array}$ & Kirsten Van Baarsen & Netherlands \\
\hline
\end{tabular}




\begin{tabular}{|c|c|c|c|c|}
\hline Pub Number & Topic & Abstract Title & Presenter & Country \\
\hline $\mathrm{P}-058$ & Hydrocephalus & $\begin{array}{l}\text { An audit of endoscopic third } \\
\text { ventriculostomy (ETV) in a } \\
\text { regional paediatric neuro- } \\
\text { surgical centre assessing the } \\
\text { accuracy and feasibility of } \\
\text { ETV success score (ETVss) }\end{array}$ & Dr Stefan Yordanov & United Kingdom \\
\hline P-059 & Hydrocephalus & $\begin{array}{l}\text { Comparison of different } \\
\text { strategies in the prevention } \\
\text { of slit ventricle syndrome in } \\
\text { pediatric hydrocephalus }\end{array}$ & Sheng Che Chou & Taiwan \\
\hline P-060 & Hydrocephalus & $\begin{array}{l}\text { Long term follow up more than } \\
2 \text { decades of patients who } \\
\text { underwent endoscopic third } \\
\text { ventriculostomy in a single } \\
\text { institute }\end{array}$ & Kazuaki Shimoji & Japan \\
\hline P-061 & Hydrocephalus & $\begin{array}{l}\text { Assessment of possibility of } \\
\text { ventriculoperitoneal shunt } \\
\text { removal and necessity of } \\
\text { endoscopic third ventriculo- } \\
\text { stomy by distal shunt exter- } \\
\text { nalization }\end{array}$ & Masahiro Nonaka & Japan \\
\hline P-062 & Hydrocephalus & $\begin{array}{l}\text { Endoscopic Third Ventriculo- } \\
\text { stomy and Endoscopic Fen- } \\
\text { estration in Multiloculated } \\
\text { Hydrocephalus and Allergic } \\
\text { Reaction to VP Shunt: A } \\
\text { Case Report }\end{array}$ & Achmad Kurniawan & Indonesia \\
\hline P-063 & Hydrocephalus & $\begin{array}{l}\text { Treatment of fetal hydrocepha- } \\
\text { lus by ventriculoamniotic } \\
\text { shunting: case report and } \\
\text { literature review }\end{array}$ & Shuo Gu & China \\
\hline P-064 & Hydrocephalus & $\begin{array}{l}\text { A Mathematical Model to } \\
\text { Simulate Intracranial Pressure } \\
\text { and Unified Interpretation of } \\
\text { Several Disease Entities }\end{array}$ & Jingsheng Wang & China \\
\hline P-065 & Hydrocephalus & $\begin{array}{l}\text { Stent and catheter placement } \\
\text { with an intraluminal endo- } \\
\text { scope in } 100 \text { cases }\end{array}$ & Joachim Oertel & Germany \\
\hline P-066 & Hydrocephalus & $\begin{array}{l}\text { Application of adjustable dif- } \\
\text { ferential and antigravitational } \\
\text { valve in pediatric hydro- } \\
\text { cephalus }\end{array}$ & Joachim Oertel & Germany \\
\hline P-067 & Hydrocephalus & $\begin{array}{l}\text { The impact of aetiology on } \\
\text { ventriculoperitoneal shunt } \\
\text { failure in patients with hydro- } \\
\text { cephalus }\end{array}$ & Geraint Sunderland & United Kingdom \\
\hline P-068 & Hydrocephalus & $\begin{array}{l}\text { Telemetric brain pressure } \\
\text { monitoring in perdiatric } \\
\text { patients }\end{array}$ & Joachim Oertel & Germany \\
\hline P-069 & Hydrocephalus & $\begin{array}{l}\text { Neurodevelopmental Outcome } \\
\text { in Children with Congenital } \\
\text { Hydrocephalus Who Under- } \\
\text { went Ventriculoperitoneal } \\
\text { Shunt: A Meta-Analysis }\end{array}$ & Danny Halim & Indonesia \\
\hline P-070 & Hydrocephalus & $\begin{array}{l}\text { Therapy paradigm with the } \\
\text { programmable gravitational } \\
\text { valves (proSA) in pediatric } \\
\text { hydrocephalic patients }\end{array}$ & Andreas Schaumann & Germany \\
\hline
\end{tabular}




\begin{tabular}{|c|c|c|c|c|}
\hline Pub Number & Topic & Abstract Title & Presenter & Country \\
\hline$\overline{\mathrm{P}-071}$ & Hydrocephalus & $\begin{array}{l}\text { Telemetric intracranial pressure } \\
\text { measurement in CSF circula- } \\
\text { tion disturbances: a retrospec- } \\
\text { tive single-center study }\end{array}$ & Valentina Pennacchietti & Germany \\
\hline P-072 & Hydrocephalus & $\begin{array}{l}\text { Hydrocephalus communicating } \\
\text { associated to primary spinal } \\
\text { primitive neuroectodermal } \\
\text { tumor maybe extraneural } \\
\text { metastases and intracranial } \\
\text { seeding in a child: a case } \\
\text { report }\end{array}$ & Abraham Ibarra De La Torre & Mexico \\
\hline P-073 & Hydrocephalus & $\begin{array}{l}\text { The incidence of infection- } \\
\text { related-etiology and perive- } \\
\text { ntricular edema in pediatric } \\
\text { hydrocephalus patients with } \\
\text { history of seizure: A possible } \\
\text { pathophysiology of seizure in } \\
\text { hydrocephalus }\end{array}$ & Danny Halim & Indonesia \\
\hline P-074 & Hydrocephalus & $\begin{array}{l}\text { Proposal of new classification } \\
\text { system for multiloculated } \\
\text { hydrocephalus }\end{array}$ & Sergei Kim & Russia \\
\hline P-075 & Hydrocephalus & $\begin{array}{l}\text { Cognitive outcomes in post- } \\
\text { haemorrhagic hydrocephalus } \\
\text { are more important to parents } \\
\text { than surgical outcomes: a } \\
\text { patient and public involve- } \\
\text { ment study }\end{array}$ & Saniya Mediratta & United Kingdom \\
\hline P-076 & Hydrocephalus & $\begin{array}{l}\text { Open versus Laparoscopic } \\
\text { Ventriculoperitoneal Shunt } \\
\text { Placement in Children: } \\
\text { An international Survey of } \\
\text { Practice. }\end{array}$ & Linus Ruf & Switzerland \\
\hline P-077 & Hydrocephalus & $\begin{array}{l}\text { Open versus Laparoscopic } \\
\text { Distal Ventriculoperitoneal } \\
\text { Shunt Placement in Children: } \\
\text { A Systematic Review and } \\
\text { Meta-Analysis. }\end{array}$ & Ladina Greuter & Switzerland \\
\hline P-078 & Infection & $\begin{array}{l}\text { Value of intraoperative Cer- } \\
\text { ebro-Spinal fluid sampling } \\
\text { during ventriculo-peritoneal } \\
\text { shunt for congenital hydro- } \\
\text { cephalus }\end{array}$ & $\begin{array}{l}\text { Dewa Pakshage Chula Kanishka } \\
\text { Ananda Lal }\end{array}$ & United Kingdom \\
\hline P-079 & Infection & $\begin{array}{l}\text { TORCH infection and associ- } \\
\text { ated anterior circulation ter- } \\
\text { ritories of brain: an observa- } \\
\text { tional study }\end{array}$ & Bipin Chaurasia & Nepal \\
\hline P-080 & Infection & $\begin{array}{l}\text { Anterior Interhemispheric } \\
\text { Transcallosal Transchoroidal } \\
\text { Fissure Approach for Third } \\
\text { Ventricle Hydatid Cyst: An } \\
\text { interesting approach to a rare } \\
\text { entity }\end{array}$ & Seyed Ali Mousavinejad & Iran \\
\hline P-081 & Infection & $\begin{array}{l}\text { Subdural empyema, an Accra } \\
\text { perspective }\end{array}$ & Teddy Tsiate Totimeh & Ghana \\
\hline P-082 & Infection & $\begin{array}{l}\text { Neuroendoscopic Irrigation for } \\
\text { Treatment of Ventriculitis } \\
\text { after Cerebrospinal Fluid } \\
\text { Diversion in Pediatrics: a } \\
\text { Feasible Option or Not? }\end{array}$ & Fitra Fitra & Indonesia \\
\hline
\end{tabular}




\begin{tabular}{|c|c|c|c|c|}
\hline Pub Number & Topic & Abstract Title & Presenter & Country \\
\hline P-083 & $\begin{array}{l}\text { Innovation and tech- } \\
\text { nology }\end{array}$ & $\begin{array}{l}\text { Ultrasonic Bone Cutting in } \\
\text { Endoscopic Craniosynostosis } \\
\text { Surgery }\end{array}$ & Edward Ahn & United States \\
\hline P-084 & $\begin{array}{l}\text { Innovation and tech- } \\
\text { nology }\end{array}$ & $\begin{array}{l}\text { Application of Soft tissue } \\
\text { Expander in the treatment of } \\
\text { skull defect with local large } \\
\text { scalp scar }\end{array}$ & Wuqiang Che & China \\
\hline P-085 & $\begin{array}{l}\text { Innovation and tech- } \\
\text { nology }\end{array}$ & $\begin{array}{l}\text { Classification of Brain } \\
\text { Tumours Stadium in Children } \\
\text { using Recurrent Neural } \\
\text { Network (RNN) Model } \\
\text { Algorithm }\end{array}$ & Rifaldy Fajar & Indonesia \\
\hline P-086 & $\begin{array}{l}\text { Innovation and tech- } \\
\text { nology }\end{array}$ & $\begin{array}{l}\text { Surgical Planning for compli- } \\
\text { cated intracranial cyst with } \\
\text { DICOM neuroimaging 3D } \\
\text { reconstruction }\end{array}$ & Jimmy Ming Jung Chuang & Taiwan \\
\hline P-087 & $\begin{array}{l}\text { Innovation and tech- } \\
\text { nology }\end{array}$ & $\begin{array}{l}\text { Minimally-invasive Parafas- } \\
\text { cicular Surgical (MIPS) } \\
\text { Approach for the Manage- } \\
\text { ment of a Pediatric Third } \\
\text { Ventricular Ependymoma: A } \\
\text { Case Report }\end{array}$ & Marian Michael Bercu & United States \\
\hline P-088 & $\begin{array}{l}\text { Innovation and tech- } \\
\text { nology }\end{array}$ & $\begin{array}{l}\text { Navigation guided endoscopic } \\
\text { cyst aspiration and cysto-ven- } \\
\text { triculostomy with Ommaya } \\
\text { reservoir placement for symp- } \\
\text { tomatic intraparenchymal cyst } \\
\text { in Labrune syndrome }\end{array}$ & Meshal Jarebi & France \\
\hline P-089 & $\begin{array}{l}\text { Innovation and tech- } \\
\text { nology }\end{array}$ & $\begin{array}{l}\text { Evaluation effectiveness and } \\
\text { bioadaptation of various } \\
\text { types of porcine collagen } \\
\text { for duraplasty in an animal } \\
\text { model }\end{array}$ & Roni Mai & Russia \\
\hline P-090 & $\begin{array}{l}\text { Innovation and tech- } \\
\text { nology }\end{array}$ & $\begin{array}{l}\text { The impact of telephone } \\
\text { consultations due to Covid19 } \\
\text { on Paediatric Neurosurgical } \\
\text { health services }\end{array}$ & Katerina Apostolopoulou & United Kingdom \\
\hline P-091 & Moya-Moya disease & $\begin{array}{l}\text { Evaluation of Accuracy of Pre- } \\
\text { surgical Optimal Target Point } \\
\text { for Extracranial-Intracranial } \\
\text { Bypass Surgery with Three- } \\
\text { Dimensional Reconstructed } \\
\text { Image-Based Presurgical } \\
\text { Planning }\end{array}$ & Jimmy Ming Jung Chuang & Taiwan \\
\hline P-092 & Moya-Moya disease & $\begin{array}{l}\text { Multiple burr hole surgery as a } \\
\text { treatment modality for pediat- } \\
\text { ric moyamoya disease }\end{array}$ & Gaddam Sai Harish & India \\
\hline P-093 & Moya-Moya disease & $\begin{array}{l}\text { D-2-hydroxyglutaric aciduria } \\
\text { type II associated with moy- } \\
\text { amoya syndrome in a } 8 \text { year } \\
\text { old child - First report in the } \\
\text { literature }\end{array}$ & Elke Januschek & Germany \\
\hline P-094 & Moya-Moya disease & $\begin{array}{l}\text { Moyamoya Syndrome in a } \\
10 \text { year old female, Filipino } \\
\text { child: A case report }\end{array}$ & Dexter Ayoso Dimaano & Philippines \\
\hline P-095 & Moya-Moya disease & $\begin{array}{l}\text { Surgical management and } \\
\text { neuropsychological profile of } \\
\text { pediatric Moyamoya patients }\end{array}$ & Katerina Apostolopoulou & United Kingdom \\
\hline
\end{tabular}




\begin{tabular}{|c|c|c|c|c|}
\hline Pub Number & Topic & Abstract Title & Presenter & Country \\
\hline P-097 & Neuroendoscopy & $\begin{array}{l}\text { Surgical Treatment and } \\
\text { Outcome of Posterior Fossa } \\
\text { Arachnoid Cysts in Infants }\end{array}$ & Jehuda Soleman & Switzerland \\
\hline P-098 & Neuroendoscopy & $\begin{array}{l}\text { Endoscopic third ventriculos- } \\
\text { tomy in patients with shunt } \\
\text { malfunction: single centre } \\
\text { analisys }\end{array}$ & Oleksandr S. Voloshchuk & Ukraine \\
\hline P-099 & Neuro-Oncology & $\begin{array}{l}\text { Multidisciplinary management } \\
\text { of spinal tumors in pediatric } \\
\text { age. } 10 \text { year experience }\end{array}$ & Garcia Gonzalez Oscar & Mexico \\
\hline P-100 & Neuro-Oncology & $\begin{array}{l}\text { Utility of Squash Cytology in } \\
\text { Intraoperative Diagnosis of } \\
\text { Pediatric Central Nervous } \\
\text { System Tumors: A Prospec- } \\
\text { tive Study of } 31 \text { Cases }\end{array}$ & Shashank Ravindra Ramdurg & India \\
\hline P-101 & Neuro-Oncology & $\begin{array}{l}\text { Role of DTI in optochiasmatic } \\
\text { glioma }\end{array}$ & Manas Panigrahi & India \\
\hline P-102 & Neuro-Oncology & $\begin{array}{l}\text { ASXL1 expression was } \\
\text { associated with prognosis of } \\
\text { intracranial ependymoma in } \\
\text { children }\end{array}$ & Yu Jianzhong & China \\
\hline P-103 & Neuro-Oncology & $\begin{array}{l}\text { Pediatric skull base tumors in a } \\
\text { single center: a management } \\
\text { challenge }\end{array}$ & Ricardo Santos De Oliveira & Brazil \\
\hline P-104 & Neuro-Oncology & $\begin{array}{l}\text { Apoplexy of atypical teratoid/ } \\
\text { rhabdoid tumor in the pineal } \\
\text { region after Ommaya reser- } \\
\text { voir drainage }\end{array}$ & Chenran Zhang & China \\
\hline P-105 & Neuro-Oncology & $\begin{array}{l}\text { 5-aminolevulinic acid-guided } \\
\text { surgery for recurrent } \\
\text { supratentorial paediatric CNS } \\
\text { neoplasms }\end{array}$ & Jason Labuschagne & South Africa \\
\hline P-106 & Neuro-Oncology & $\begin{array}{l}\text { Hypoxia- and Dexametha- } \\
\text { sone-dependent interac- } \\
\text { tion between HIF1 } \alpha \text { and } \\
\text { glucocorticoid receptor leads } \\
\text { to cytoplasmic retention and } \\
\text { degradation of glucocorti- } \\
\text { coid receptor in pituitary } \\
\text { adenomas }\end{array}$ & Chenran Zhang & China \\
\hline P-107 & Neuro-Oncology & $\begin{array}{l}\text { The spectrum of pediatric skull } \\
\text { tumors }\end{array}$ & Martina Messing Jünger & Germany \\
\hline P-108 & Neuro-Oncology & $\begin{array}{l}\text { A Thirteen-Year Review of } \\
\text { Central Nervous System } \\
\text { (CNS) Tumours in Infants at } \\
\text { a Single Centre }\end{array}$ & Isabel Tulloch & United Kingdom \\
\hline P-109 & Neuro-Oncology & $\begin{array}{l}\text { Transsphenoidal Surgery in } \\
\text { Pituitary Tumors among the } \\
\text { Young }\end{array}$ & Sivashanmugam Dhandapani & India \\
\hline P-110 & Neuro-Oncology & $\begin{array}{l}\text { Cerebellar swelling after } \\
\text { surgery for medulloblas- } \\
\text { toma with leptomeningeal } \\
\text { dissemination in children. A } \\
\text { case-based update }\end{array}$ & Stella Irene & France \\
\hline P-111 & Neuro-Oncology & $\begin{array}{l}\text { Pediatric posterior fossa epend- } \\
\text { ymomas: Still à challenge for } \\
\text { pediatric neurosurgeons and } \\
\text { oncologists }\end{array}$ & Rel Gerald Boukaka & France \\
\hline
\end{tabular}




\begin{tabular}{|c|c|c|c|c|}
\hline Pub Number & Topic & Abstract Title & Presenter & Country \\
\hline P-112 & Neuro-Oncology & $\begin{array}{l}\text { Long term surgical outcome } \\
\text { of chilhood and adolescent } \\
\text { pilocytic astrocytoma }\end{array}$ & Suchanda Bhattacharjee & India \\
\hline P-113 & Neuro-Oncology & $\begin{array}{l}\text { Machine-learning inference } \\
\text { may predict quality of life } \\
\text { subgroups of Adamantinoma- } \\
\text { tous Craniopharyngioma }\end{array}$ & Astrid C Hengartner & United States \\
\hline P-114 & Neuro-Oncology & $\begin{array}{l}\text { Prevalence of human immu- } \\
\text { nodeficiency virus infection } \\
\text { in brain glioma patients: Is } \\
\text { the virus protective from } \\
\text { gliomas? }\end{array}$ & Luxwell Jokonya & Zimbabwe \\
\hline P-115 & Neuro-Oncology & $\begin{array}{c}\text { Pediatric Pituitary Adenomas - } \\
\text { A Different Clinical Entity? }\end{array}$ & Mohamed Amjad Jamaluddin & India \\
\hline P-116 & Neuro-Oncology & $\begin{array}{l}\text { Atypical Teratoid Rhabdoid } \\
\text { Tumours (ATRTs): a 23-year } \\
\text { institutional Experience }\end{array}$ & Adikarige Haritha Dulanka Silva & United Kingdom \\
\hline P-117 & Neuro-Oncology & $\begin{array}{l}\text { Surgical management of } \\
\text { choroid plexus tumors in } \\
\text { children: A cohort series of } \\
35 \text { cases }\end{array}$ & Tai Tong Wong & Taiwan \\
\hline P-118 & Neuro-Oncology & $\begin{array}{l}\text { A Retrospective Study of Pedi- } \\
\text { atric Brain Tumors from a } \\
\text { Tertiary Care in South China }\end{array}$ & Huirong Mai & China \\
\hline P-119 & Neuro-Oncology & $\begin{array}{l}\text { Endoscopic Ommaya reservoir } \\
\text { placement via third ven- } \\
\text { tricular stoma for intrathecal } \\
\text { methotrexate administration } \\
\text { for subarachnoid dissemina- } \\
\text { tion of medulloblastoma }\end{array}$ & Azumi Kaneoka & Japan \\
\hline P-120 & Neuro-Oncology & $\begin{array}{l}\text { Endoscopic extended trans- } \\
\text { sphenoidal surgery for newly } \\
\text { diagnosed paediatric crani- } \\
\text { opharyngiomas }\end{array}$ & Michael Amoo & Ireland \\
\hline $\mathrm{P}-121$ & Neuro-Oncology & $\begin{array}{l}\text { Pediatric vestibular schwanno- } \\
\text { mas without clinical evidence } \\
\text { of neurofibromatosis: Micro- } \\
\text { surgical experience of } 18 \\
\text { consecutive patients }\end{array}$ & Ken Matsushima & Japan \\
\hline $\mathrm{P}-122$ & Neuro-Oncology & $\begin{array}{l}\text { Mapping the research land- } \\
\text { scape in cerebellar mutism } \\
\text { and posterior fossa syndrome }\end{array}$ & Aaron Lawson McLean & Germany \\
\hline P-123 & Neuro-Oncology & $\begin{array}{l}\text { The prevalence of Cancer } \\
\text { Predisposition Syndromes in } \\
\text { Pediatric High Grade Glioma } \\
\text { in Saudi Arabia }\end{array}$ & Mahmoud Taha & Saudi Arabia \\
\hline P-124 & Neuro-Oncology & $\begin{array}{l}\text { Central Nervous System } \\
\text { Lymphoblastic Chloromas: } \\
\text { Management of refractory } \\
\text { Acute Lymphoblastic Leuke- } \\
\text { mia in Pediatric Patients after } \\
\text { Stem Cell Transplantation }\end{array}$ & Aleka Nicole Scoco & United States \\
\hline P-125 & Neuro-Oncology & $\begin{array}{l}\text { A Rare Case of Brain Metas- } \\
\text { tasis of Embryonal Rhabdo- } \\
\text { myosarcoma in 7-year old } \\
\text { Girl: Case Report }\end{array}$ & Nikkita Ihsan & Indonesia \\
\hline P-126 & Neuro-Oncology & $\begin{array}{l}\text { The Landscape of Neuro- } \\
\text { oncology in East Africa: A } \\
\text { Review of Published Records }\end{array}$ & Joseline Haizel Cobbina & United States \\
\hline
\end{tabular}




\begin{tabular}{|c|c|c|c|c|}
\hline Pub Number & Topic & Abstract Title & Presenter & Country \\
\hline P-127 & Neuro-Oncology & $\begin{array}{l}\text { Incidental Pituitary Cysts } \\
\text { in Children: Does Growth } \\
\text { Hormone Treatment Affect } \\
\text { Cyst Size? }\end{array}$ & Brandon G Rocque & United States \\
\hline P-128 & $\begin{array}{l}\text { Neurotrauma / Critical } \\
\text { Care }\end{array}$ & $\begin{array}{l}\text { Role of routine post-operative } \\
\text { CT Brain following evacua- } \\
\text { tion of extradural haematoma } \\
\text { in Children; a single-centre } \\
\text { experience }\end{array}$ & Michael Oluwaseun Amoo & Ireland \\
\hline P-129 & $\begin{array}{l}\text { Neurotrauma / Critical } \\
\text { Care }\end{array}$ & $\begin{array}{l}\text { Optimizing transfusion-related } \\
\text { postoperative outcomes in } \\
\text { craniosynostosis repair }\end{array}$ & Robin Yang & United States \\
\hline P-130 & $\begin{array}{l}\text { Neurotrauma / Critical } \\
\text { Care }\end{array}$ & $\begin{array}{l}\text { Traumatic atlantooccipital dis- } \\
\text { traction - prevention by using } \\
\text { finite element models }\end{array}$ & Cordula Scherer & Switzerland \\
\hline P-131 & $\begin{array}{l}\text { Neurotrauma / Critical } \\
\text { Care }\end{array}$ & $\begin{array}{l}\text { Establishment of the Dutch } \\
\text { Pediatric Craniectomy } \\
\text { and Cranioplasty Registry } \\
\text { (DPCCR) - A Prospective } \\
\text { Multicenter Open Registry }\end{array}$ & Vita M. Klieverik & Netherlands \\
\hline P-132 & $\begin{array}{l}\text { Neurotrauma / Critical } \\
\text { Care }\end{array}$ & $\begin{array}{l}\text { Extradural hematoma in } \\
\text { children: experience of } \\
\text { Centre Hospitalier National } \\
\text { Universitaire de Fann at } \\
\text { Dakar- Sénégal }\end{array}$ & Rel Gerald Boukaka & France \\
\hline P-134 & $\begin{array}{l}\text { Neurotrauma / Critical } \\
\text { Care }\end{array}$ & $\begin{array}{l}\text { Efficacy of Hinge and Floating } \\
\text { Decompressive Craniotomy } \\
\text { for Infantile Acute Subdural } \\
\text { Hematoma }\end{array}$ & Tae Kyun Kim & Japan \\
\hline P-135 & $\begin{array}{l}\text { Neurotrauma / Critical } \\
\text { Care }\end{array}$ & $\begin{array}{l}\text { Penetrating Brain Injury In } \\
\text { Pediatrics With Double } \\
\text { Approach Of Sub-Frontal } \\
\text { Craniotomy And Endoscopic } \\
\text { Endonasal For Anterior Skull } \\
\text { Base Reconstruction: A Case } \\
\text { Report }\end{array}$ & Andhika Tomy Permana & Indonesia \\
\hline P-136 & $\begin{array}{l}\text { Neurotrauma / Critical } \\
\text { Care }\end{array}$ & $\begin{array}{l}\text { Endolumbal Ozone and } \\
\text { Pyracetam Insufflation in the } \\
\text { Postoperative Period after } \\
\text { Evacuation of Posttraumatic } \\
\text { Arachnoid Cysts }\end{array}$ & Mansur A. Aliev & Uzbekistan \\
\hline P-137 & $\begin{array}{l}\text { Neurotrauma / Critical } \\
\text { Care }\end{array}$ & $\begin{array}{l}\text { Traumatic cerebrospinal fluid } \\
\text { (CSF) Leak in infants }\end{array}$ & Yahya H. Khormi & Saudi Arabia \\
\hline P-138 & $\begin{array}{l}\text { Neurotrauma / Critical } \\
\text { Care }\end{array}$ & $\begin{array}{l}\text { Acidification of the intracel- } \\
\text { lular environment during an } \\
\text { acute period after a con- } \\
\text { cussion according to MR } \\
\text { spectroscopy }\end{array}$ & Petr Bulanov & Russia \\
\hline P-139 & $\begin{array}{l}\text { Neurotrauma / Critical } \\
\text { Care }\end{array}$ & $\begin{array}{l}\text { Vagus nerve stimulation in } \\
\text { recovery treatment after } \\
\text { severe TBI. }\end{array}$ & Zhanna Semenova & Russia \\
\hline P-140 & $\begin{array}{l}\text { Neurotrauma / Critical } \\
\text { Care }\end{array}$ & $\begin{array}{l}\text { Impaired cerebral autoregula- } \\
\text { tion following cardiovascular } \\
\text { resuscitation correlates to } \\
\text { unfavorable neurological out- } \\
\text { come in pediatric patients }\end{array}$ & Martin Ulrich Schuhmann & Germany \\
\hline P-141 & Occult tethered cord & $\begin{array}{l}\text { Urological outcomes of spinal } \\
\text { lipomas after prophylactic } \\
\text { and "rescue" untethering in } \\
\text { children }\end{array}$ & Garcia Gonzalez Oscar & Mexico \\
\hline
\end{tabular}




\begin{tabular}{|c|c|c|c|c|}
\hline Pub Number & Topic & Abstract Title & Presenter & Country \\
\hline $\mathrm{P}-142$ & Occult tethered cord & $\begin{array}{l}\text { Posttubercular Arachnoid Cyst } \\
\text { and Syringomyelia with } \\
\text { Severe Spastic Paraplegia: } \\
\text { case report and review of } \\
\text { literature }\end{array}$ & Hsin Hung Chen & Taiwan \\
\hline P-143 & Other & $\begin{array}{l}\text { Explore the role of CR1 in } \\
\text { Alzheimer's disease }\end{array}$ & Xichen Zhu & China \\
\hline P-144 & Other & $\begin{array}{l}\text { The surgical treatments of non- } \\
\text { syndromic craniosynostosis } \\
\text { in children }\end{array}$ & Can Do Thanh Dang & Vietnam \\
\hline P-145 & Other & $\begin{array}{l}\text { Cerebral cavernomas in the } \\
\text { pediatric age: about } 5 \text { cases }\end{array}$ & Nesrine Jemel & Tunisia \\
\hline P-146 & Other & $\begin{array}{l}\text { Technical Note: Osteoblastic } \\
\text { Telescopic Spiral Tecnique } \\
\text { for Craniosynostosis Cor- } \\
\text { rection }\end{array}$ & Dunia Patricia Quiroga Angulo & Colombia \\
\hline P-147 & Other & $\begin{array}{l}\text { Diagnosis of Alzheimer's Dis- } \\
\text { ease using the Expert System } \\
\text { Based on Tsukamoto Fuzzy } \\
\text { Algorithm }\end{array}$ & Rifaldy Fajar & Indonesia \\
\hline P-148 & Other & $\begin{array}{l}\text { Pancraniosynostosis: Individu- } \\
\text { alized Treatment }\end{array}$ & Dhaval Shukla & India \\
\hline P-149 & Other & $\begin{array}{l}\text { Research Productivity of } \\
\text { Pediatric Neurosurgeons in } \\
\text { Canada }\end{array}$ & Farhan Mahmood & Canada \\
\hline $\mathrm{P}-150$ & Other & $\begin{array}{l}\text { Selective delivery of bioactive } \\
\text { compounds through stimuli- } \\
\text { responsive nanocarriers as } \\
\text { a noninvasive treatment for } \\
\text { nonsyndromic craniosynos- } \\
\text { tosis }\end{array}$ & Gianpiero Tamburrini & Italy \\
\hline $\mathrm{P}-151$ & Other & $\begin{array}{l}\text { Social Media Trends Among } \\
\text { Pediatric Neurosurgeons }\end{array}$ & Sauson Soldozy & United States \\
\hline $\mathrm{P}-152$ & Other & $\begin{array}{l}\text { Development of the Alberta } \\
\text { Infant Motor Scale digital } \\
\text { application: towards better } \\
\text { neurological assessment of } \\
\text { in-risk children }\end{array}$ & Renata Jucá & Brazil \\
\hline $\mathrm{P}-153$ & Other & $\begin{array}{l}\text { Imaging of Pediatric Calvarial } \\
\text { Lesions }\end{array}$ & Peter Kalina & United States \\
\hline $\mathrm{P}-154$ & Other & $\begin{array}{l}\text { Intraoperative Measurement of } \\
\text { Intracranial Pressure During } \\
\text { Cranial Vault Remodeling } \\
\text { in Children with Craniosyn- } \\
\text { ostosis }\end{array}$ & Dhaval Shukla & India \\
\hline $\mathrm{P}-155$ & Other & $\begin{array}{l}\text { Cranial Fasciitis after cranial } \\
\text { distraction in craniosynosto- } \\
\text { sis patient }\end{array}$ & Ahmed Aly & United Kingdom \\
\hline P-156 & Other & $\begin{array}{l}\text { Blood Transfusion and Donor } \\
\text { Exposure in Surgical Man- } \\
\text { agement of Trigonocephaly } \\
\text { Patients - The Alder Hey } \\
\text { Experience }\end{array}$ & Benjamin Rapaport & United Kingdom \\
\hline P-157 & Spine & $\begin{array}{l}\text { A Rapid prototype Patient- } \\
\text { Specific Navigational Drill } \\
\text { Template for Pediatric } \\
\text { Posterior Transpedicle Screw } \\
\text { Placement }\end{array}$ & Jimmy Ming Jung Chuang & Taiwan \\
\hline
\end{tabular}




\begin{tabular}{|c|c|c|c|c|}
\hline Pub Number & Topic & Abstract Title & Presenter & Country \\
\hline$\overline{\mathrm{P}-158}$ & Spine & $\begin{array}{l}\text { Functional Outcome of Chil- } \\
\text { dren with Spinal Cord Injury }\end{array}$ & Manju Dhandapani & India \\
\hline P-159 & Spine & $\begin{array}{l}\text { Systematic Review on use and } \\
\text { efficacy of Selective Dorsal } \\
\text { Rhizotomy (SDR) for the } \\
\text { Management of Spasticity in } \\
\text { Adult Patients }\end{array}$ & Pramath Kakodar & Canada \\
\hline P-160 & Spine & $\begin{array}{l}\text { Anomaly-related pathological } \\
\text { atlantoaxial displacement in } \\
\text { pediatric patients }\end{array}$ & Olga M Sergeenko & Russia \\
\hline P-161 & Spine & $\begin{array}{l}\text { Atlantoaxial dislocation due to } \\
\text { os odontoideum in patients } \\
\text { with Down's syndrome: } \\
\text { literature review and case } \\
\text { reports }\end{array}$ & Olga M Sergeenko & Russia \\
\hline P-162 & Spine & $\begin{array}{l}\text { Early Experience in Pediatric } \\
\text { Spine Infection: Case Report }\end{array}$ & Astri Avianti & Indonesia \\
\hline P-163 & Spine & $\begin{array}{l}\text { Risk assessment of jatrogenic } \\
\text { motor disorders in surgical } \\
\text { correction of spinal deformi- } \\
\text { ties of various etiologies }\end{array}$ & Polina Ochirova & Russia \\
\hline P-164 & Spine & $\begin{array}{l}\text { Building consensus for the } \\
\text { medical management of } \\
\text { children with moderate and } \\
\text { severe acute spinal cord } \\
\text { injury (SCI): A modified } \\
\text { Delphi study }\end{array}$ & Richard Anderson & United States \\
\hline P-165 & Spine & $\begin{array}{l}\text { Outcome of DermabondTM } \\
\text { PrineoTM for wound closure } \\
\text { in pediatric spine surgery }\end{array}$ & Jehuda Soleman & Switzerland \\
\hline P-166 & Vascular & $\begin{array}{l}\text { Pediatric Intracranial Giant } \\
\text { Aneurysm in a } 10 \text {-year old } \\
\text { Female: A Case Report }\end{array}$ & Emmanuel Jr Escarlan Albano & Philippines \\
\hline P-167 & Vascular & $\begin{array}{l}\text { Multimodality Management of } \\
\text { Pediatric Brain Arteriovenous } \\
\text { Malformations in Children: A } \\
7 \text { year Review }\end{array}$ & Olufemi Ajani & Canada \\
\hline P-168 & Vascular & $\begin{array}{l}\text { A case of progressive multiple } \\
\text { cerebral aneurysms in a child }\end{array}$ & Koichiro Sakamoto & Japan \\
\hline P-169 & Vascular & $\begin{array}{l}\text { Ruptured middle cerebral } \\
\text { artery aneurysm in infancy: A } \\
\text { case report }\end{array}$ & Yuhei Takido & Japan \\
\hline $\mathrm{P}-170$ & Vascular & $\begin{array}{l}\text { Combined Indirect Revascu- } \\
\text { larization and Stereotactic } \\
\text { Radiosurgery to Manage } \\
\text { Cerebral Proliferative Angi- } \\
\text { opathy }\end{array}$ & Yu Ning Chen & Taiwan \\
\hline $\mathrm{P}-171$ & Vascular & $\begin{array}{l}\text { Congenital Dural Arte- } \\
\text { riovenous Fistula: A Case } \\
\text { Report and Review of Imag- } \\
\text { ing Findings }\end{array}$ & Muhamad Faisal Akbar Thamrin & Indonesia \\
\hline P-172 & Vascular & $\begin{array}{l}\text { Cerebral Vasospasm after } \\
\text { Endoscopic Fenestration of a } \\
\text { Middle Fossa Arachnoid Cyst } \\
\text { by a Child }\end{array}$ & Anna Prajsnar Borak & Germany \\
\hline
\end{tabular}

Publisher's Note Springer Nature remains neutral with regard to jurisdictional claims in published maps and institutional affiliations. 